University of Rhode Island

\title{
DigitalCommons@URI
}

Open Access Dissertations

2018

\section{H-Bonding Catalyst Systems for the Ring-Opening Polymerization of Cyclic Esters and Carbonates}

Timothy J. Bannin

University of Rhode Island, timbannin@gmail.com

Creative Commons License

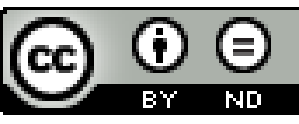

This work is licensed under a Creative Commons Attribution-No Derivative Works 4.0 License.

Follow this and additional works at: http://digitalcommons.uri.edu/oa_diss

Terms of Use

All rights reserved under copyright.

\section{Recommended Citation}

Bannin, Timothy J., "H-Bonding Catalyst Systems for the Ring-Opening Polymerization of Cyclic Esters and Carbonates" (2018).

Open Access Dissertations. Paper 711.

http://digitalcommons.uri.edu/oa_diss/711

This Dissertation is brought to you for free and open access by DigitalCommons@URI. It has been accepted for inclusion in Open Access Dissertations by an authorized administrator of DigitalCommons@URI. For more information, please contact digitalcommons@etal.uri.edu. 
H-BONDING CATALYST SYSTEMS FOR THE RING-OPENING POLYMERIZATION OF CYCLIC ESTERS AND CARBONATES

BY

TIMOTHY J. BANNIN

A THESIS SUBMITTED IN PARTIAL FULFILLMENT OF THE REQUIREMENTS FOR THE DEGREE OF DOCTOR OF PHILOSOPHY

IN

CHEMISTRY

UNIVERSITY OF RHODE ISLAND

2018 


\section{DOCTOR OF PHILOSOPHY DISSERTATION \\ OF}

TIMOTHY J. BANNIN

APPROVED:
Dissertation Committee:
$\begin{aligned} & \text { Major Professor: } \quad \text { Matthew K. Kiesewetter } \\ & \text { Brenton DeBoef } \\ & \\ & \text { David Heskett } \\ & \text { Nasser H. Zawia } \\ & \text { DEAN OF THE GRADUATE SCHOOL }\end{aligned}$

UNIVERSITY OF RHODE ISLAND

2018 


\section{ABSTRACT}

The overarching theme of my work pertains to the development of catalytic systems to affect the organocatalyzed ring-opening polymerization of cyclic esters and carbonates. Hydrogen bond mediated organocatalysts provide a robust and controllable synthetic route to well defined polyesters and polycarbonates. However, organocatalysts tend to be either highly selective or highly active. This research includes several projects which delve into how changing the $\mathrm{H}$ bonding catalytic systems effects rates of polymerization; specifically, when applied to the organocatalyzed ROP of $\varepsilon$-thiocaprolactone $(\mathrm{tCL}), \delta$-valerolactone $(\mathrm{VL})$, and $\varepsilon$-caprolactone (CL).

The first manuscript, "Ring-Opening Polymerization of Thiol Containing Cyclic Carbonate and Lactone Monomers: A Review", brings together decades of work applied to the ROP of cyclic carbonates and esters and is prepared for publication in Macromolecules. The background presented in manuscript 1 serves to provide the reader with some historical pretext for the following chapters included in this thesis.

The second manuscript, "Poly(thioester) by Organocatalytic Ring-Opening Polymerization", discusses the $\mathrm{H}$-bond mediated organocatalytic ROP of $\mathrm{tCL}$. Previous attempts to polymerize tCL were successful but entailed harsh conditions and high temperatures. In an effort to polymerize tCL utilizing milder synthetic routes and in a "living" manner, several H-bond accepting bases were screened; leading to a broadening of $M_{\mathrm{w}} / M_{\mathrm{n}}$. However, it was discovered when a $\mathrm{H}$-bond donating thiourea co-catalyst was paired with a $\mathrm{H}$-bond accepting base the unwanted transesterification was suppressed; implicated by the decreased $M_{w} / M_{n}$ of the base/thiourea catalyzed polymerization of $\mathrm{tCl}$ versus the same reaction catalyzed by base alone. All results and work were completed by the author and have been published in Macromolecules in 2015.

The third manuscript, "Triclocarban: Commercial Antibacterial and Highly Effective H-Bond Donating Catalyst for Ring-Opening Polymerization", discusses the application of the recently banned, antibacterial, and commercially available triclocarban (TCC) as a H-bond donating cocatalyst in the base/TCC catalyzed ROP of VL and CL. TCC was shown to be a highly effective 
urea co-catalyst when paired with a $\mathrm{H}$-bond accepting base. When applied to the ROP of VL and CL, the base/TCC mediated polymerizations proceeded in a "living" manner. This simple change from a thiourea to a urea-based $\mathrm{H}$-bond donating co-catalyst proved to be monumental for our group. Additionally, two electronically similar $\mathrm{H}$-bond donating ureas were synthesized and evaluated, mono-CC and di-CC. The urea-based co-catalysts are shown to remain highly active in hydrogen bonding solvents; unlike their thiourea based conjugates. This work was a collaborative effort and the thesis author completed all work encompassing the MTBD/nCTU ( $n$ $=2-6$ and 12) catalyzed polymerization of VL in acetone- $d_{6}$, MTBD/TCC catalyzed reaction of $\mathrm{CL}$ in benzene-d6, MTBD/di-CC and MTBD/mono-CC catalyzed ROP polymerizations of VL in benzene- $d_{6}$, and the synthesis of both the di-CC and mono-CC urea co-catalysts. These results were all published in ACS Macro Letters in 2017.

The fourth manuscript, "Bis-thiourea mediated organocatalyzed ROP of a cyclic lactone", investigates the effect that bis-thiourea $\mathrm{H}$-bond donating cocatalysts have on the ROP of VL. Several new thiourea $\mathrm{H}$-bond donating catalysts were developed and applied to the base/nCTU ( $n=2-6$ and 12) catalyzed ROP of $V L$ in an attempt to increase control and rate of the polymerization. Several bases were examined for their efficacy in the ROP of VL. The application of Michaelis-Menten enzyme kinetics to the MTBD/5CTU catalyzed ROP of VL coupled with the $\mathrm{k}_{\mathrm{ie}}$ of the DBU/5CTU catalyzed ROP of VL led to the conclusion that competitive inhibition exhibits itself. The observations and results were prepared for publication in ACS Macro Letters.

The fifth manuscript, "Stilbene Synthesis by Olefin Metathesis Reaction", changes gears a bit and proposes a new sophomore organic chemistry laboratory. Used as a sequence, Wittig then Metathesis, the student can compare and contrast not only a "non-green" and "green" synthetic approach to stilbene, but also thermodynamic versus kinetic control. However, this work specifically deals with the Gubbs 2 catalyzed metathesis of stilbene. The students conduct an easy 1.5 - 2-hour experiment and characterize their results by ${ }^{1} \mathrm{HNMR}$, IR, and meting point. This work was a collaborative effort. The thesis author and Partha Datta both optimized the metathesis reaction catalyzed by Grubbs 2 for the laboratory. A class of Advanced Organic 
Chemistry students, Partha Datta, and the thesis author shared in data collection and characterization. Overall, the organic lab was a success and was submitted for publication in the Journal of Chemical Education in 2018. 


\section{AKNOWLEDGEMENTS}

The author would like to thank his family for their support and unwavering belief in their son. $\mathrm{He}$ would also like to personally extend his appreciation and admiration for Dr. Matthew and Elizabeth Kiesewetter; they have been an invaluable source of knowledge and sarcasm. Tim would like to extend his gratitude to the Kiesewetter group members; past and present. Also, the faculty and staff of the Department of Chemistry at the University of Rhode Island have been extremely supportive during his graduate school tenure. 


\section{PREFEACE}

This dissertation is presented in manuscript format according to the guidelines of the Graduate School of the University of Rhode Island. The complete dissertation is divided into five manuscripts. The first manuscript (Chapter 1) was formatted for publication in Macromolecules with author T. Bannin. The second manuscript (Chapter 2) was published in Macromolecules in 2015 with authors T. Bannin and M. Kiesewetter. The third manuscript (Chapter 3) was published in ACS Macro Letters in 2017 with authors N. U. Dharmaratne, J. U. Pothupitiya, T. J. Bannin, O. I. Kazakov, and M. K. Kiesewetter. The fourth manuscript (Chapter 4) was prepared for publication in ACS Macro Letters with author T. Bannin. The final manuscript (Chapter 5) was submitted for publication to The Journal of Chemical Education in 2018 with authors T. J. Bannin, P. P. Datta, E. T. Kiesewetter, and M. K. Kiesewetter. 


\section{TABLE OF CONTENTS}

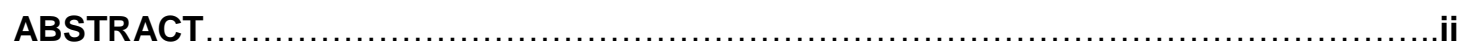

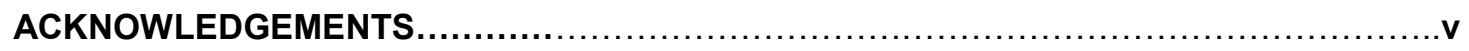

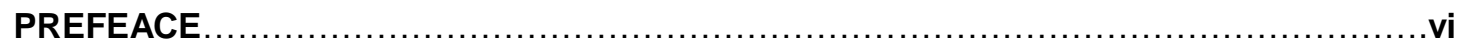

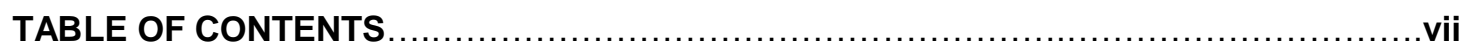

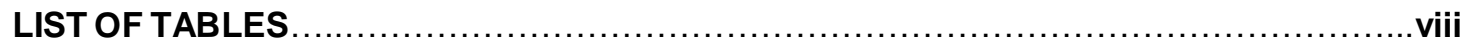

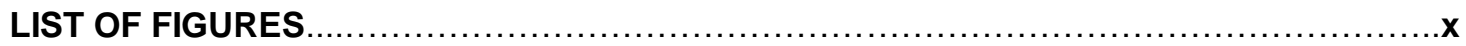

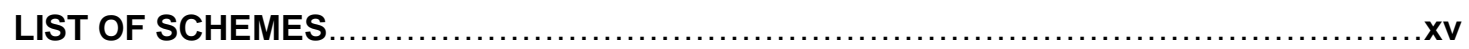

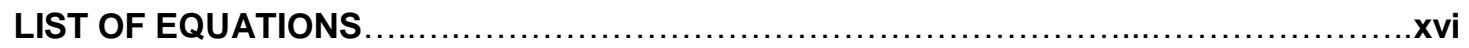

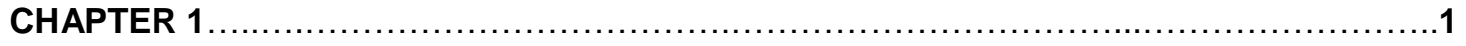

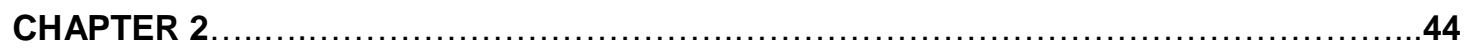

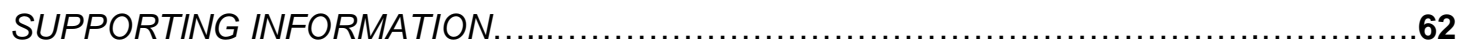

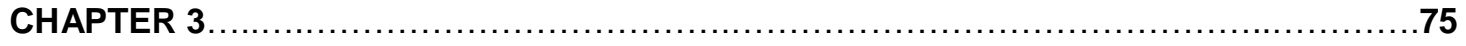

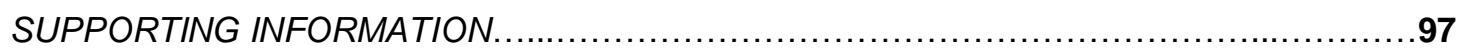

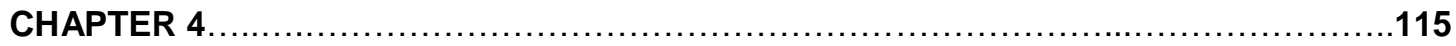

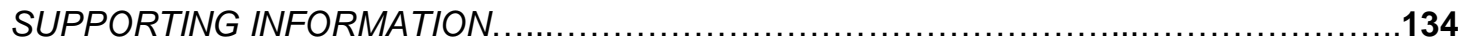

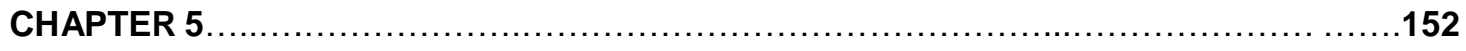

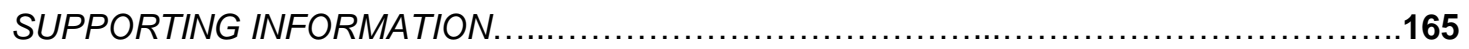




\section{LIST OF TABLES}

\section{CHAPTER 1}

Table 1. Reaction of $\omega$-halo acid chlorides with tetrathiomolybdate in solution.................8

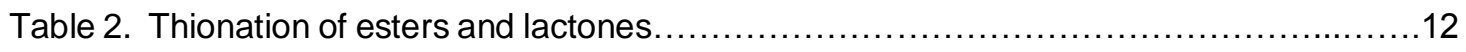

Table 3. Synthesis of thionolactones (b) and dithiolactones (d) under microwave irradiation...15

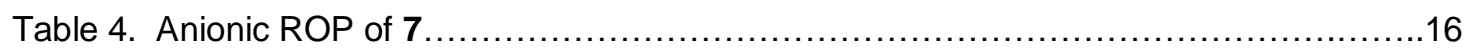

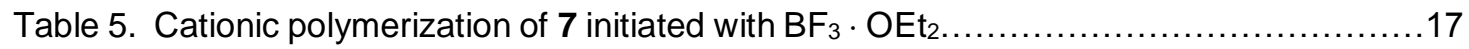

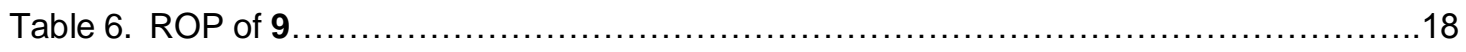

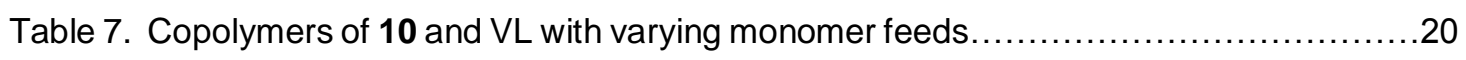

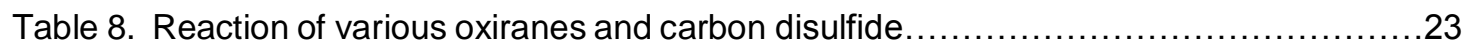

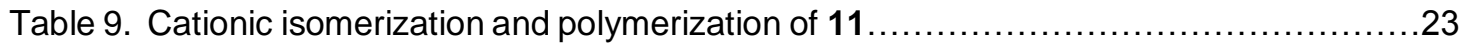

Table 10. The depolymerization of a polythiodicarbonate $\left(M_{n}=11,000, M_{w} / M_{n}=1.2\right) \ldots \ldots \ldots .25$

Table 11. Cationic ROP of 5-membered cyclic ditjiocarbonates...........................27

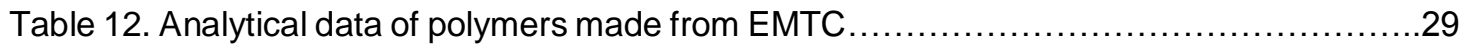

Table 13. Polymerizations of 13 initiated by acidic catalysts.............................

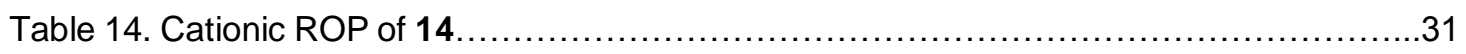

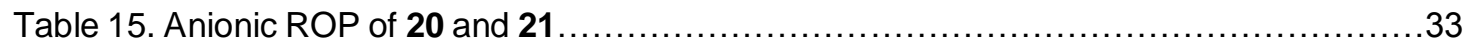

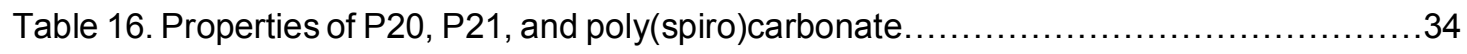

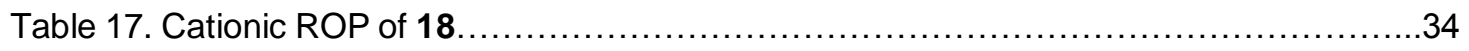

Table 18. Cationic ring-opening copolymerization of 19 with $\mathrm{CM} 1, \mathrm{CM} 2, \mathbf{1 3}$, and $14 \ldots \ldots \ldots \ldots . . .35$

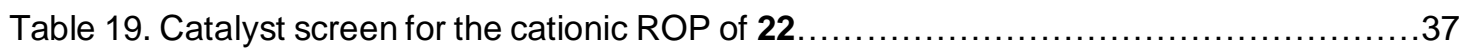

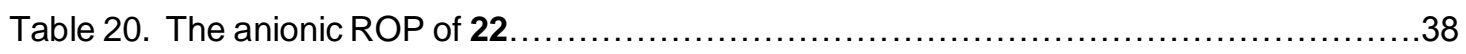

\section{CHAPTER 2}

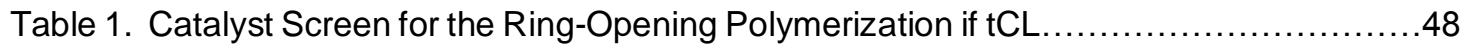

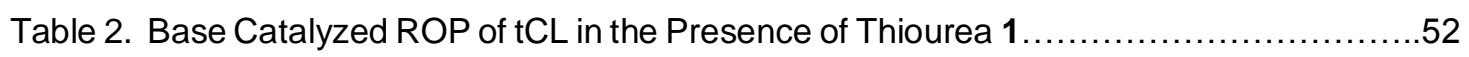

\section{CHAPTER 3}

Table 1. MTBD and TCC Catalyzed ROP of VL and CL...................................

Table 2. Solvent Screen of TCC/MTBD cocatalyzed ROP of VL $\ldots \ldots \ldots \ldots \ldots \ldots \ldots \ldots \ldots \ldots \ldots 1$ 
Table 3. Urea or Thiourea Plus MTBD Cocatalyzed ROP of VL in Acetone

Table 4. Triclocarban Plus BEMP Cocatalyzed ROP of VL and CL ........................104

Table 5. Chain Length Variation for the TCC or di-CC plus MTBD cocatalyzed ROP of VL....110

Table 6. Chain Length Variation for the TCC/MTBD cocatalyzed ROP of VL in acetone- $d_{6} \ldots . .111$

\section{CHAPTER 4}

Table 1. MTBD/3CTU catalyzed ROP of CL, VL, and BL.............................119

Table 2. Variation of the thiourea co-catalyst in the MTBD/nCTU catalyzed ROP of VL........120

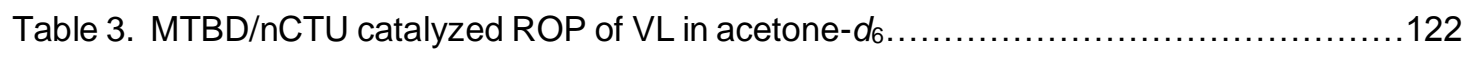

Table 4. Base screen for the base/5CTU catalyzed ROP of VL..........................123

Table S1. [M]//[I]。 screen for the MTBD/5CTU catalyzed ROP of VL .......................134

Table S2. $[\mathrm{M}]_{\mathrm{o}} /[\mathrm{l}]_{\mathrm{o}}$ screen for the DBU/5CTU catalyzed ROP of VL........................135

Table S3. Initial rate and relative initial rate of the BEMP/nCTU catalyzed ROP of VL..........145

Table S4. Initial rate and relative initial rate of the MTBD/nCTU catalyzed ROP of VL.........145

Table S5. Initial rate and relative initial rate of the DBU/nCTU catalyzed ROP of VL.........145 


\section{LIST OF FIGURES}

\section{CHAPTER 1}

Figure 1. Monomers with abbreviations used in this paper...................................

Figure 2. Van't Hoff plot for the TBD catalyzed ROP of $\varepsilon$-tCL............................

Figure 3. Esters, thionoesters, lactones, and thionolactones...........................11

\section{CHAPTER 2}

Figure 1. Evolution of percent conversion vs $M_{\mathrm{n}}$ (blue) and $M_{\mathrm{w}} / M_{\mathrm{n}}$ (red) for the ROP of tCL (1M) from octadecylthiol $(0.02 \mathrm{M})$ in chloroform catalyzed by (upper) $0.05 \mathrm{M} \mathrm{MTBD;}$; and (lower) 0.05 M MTBD and $0.05 \mathrm{M} 1$.

Figure S1. $M_{\mathrm{n}}$ (blue) and $M_{\mathrm{w}} / M_{\mathrm{n}}$ (red) vs. \% Conversion for the MTBD catalyzed ROP of $\varepsilon$-tCL;

$[\mathrm{M}] \mathrm{0} /[\mathrm{I}]_{0}=100$.

Figure S2. $M_{\mathrm{n}}$ (blue) and $M_{\mathrm{w}} / M_{\mathrm{n}}$ (orange) vs. \% Conversion for the DBU catalyzed ROP of $\varepsilon$ tCL. 62

Figure S3. Two views of a calculated equilibrium geometry (Spartan '14, DFT B3LYP 6-31G*, gas phase) with calculated dipole moment vector of (upper) $\mathrm{CL}$ and, (lower) $\varepsilon$-tCL

Figure S4. $M_{\mathrm{n}}$ (blue) and $M_{\mathrm{w}} / M_{\mathrm{n}}$ (orange) vs. \% Conversion for the DBU/1 catalyzed ROP of $\varepsilon$ -

$\mathrm{tCL}$ 64

Figure S5. GPC Trace of MTBD/1 initiated by octadecylthiol; RI detector (above) and MTBD/1 initiated by 1 -pyrenebutanol; $\mathrm{RI}$ in grey and UV (300 nm) orange (below). .64

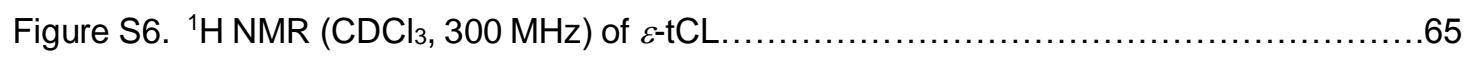

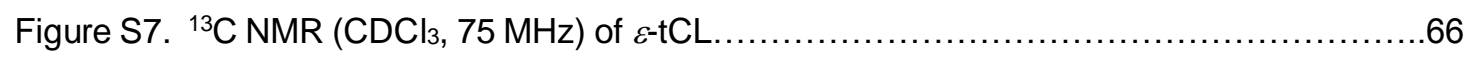

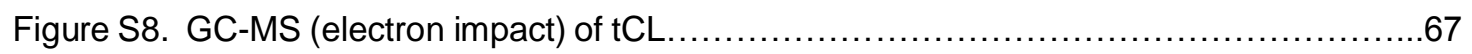

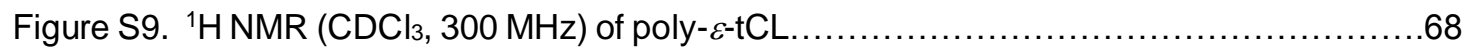

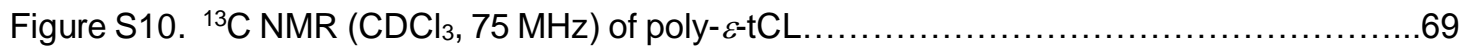

Figure S11. Equation $22,27-29$ and plot used to determine the binding constant, Keq, between $\varepsilon$ -

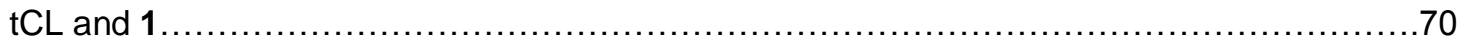

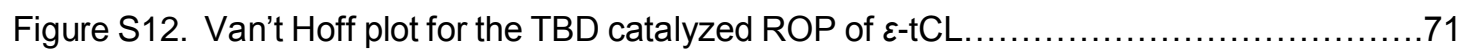

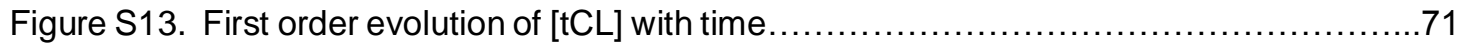




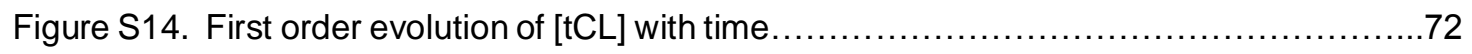

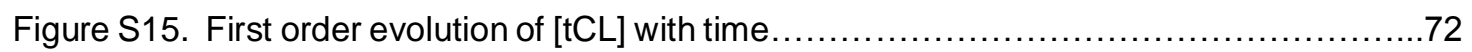

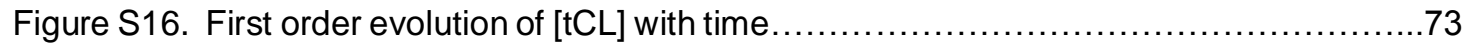

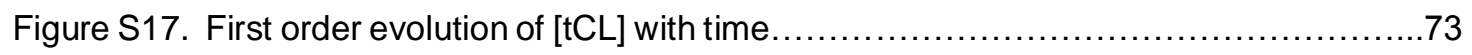

Figure S18. Upfield portions of ${ }^{1} \mathrm{H}$ NMR spectra $\left(\mathrm{C}_{6} \mathrm{D}_{6}, 300 \mathrm{MHz}\right)$ of (lower) $100 \mathrm{mM}$ octadecylthiol of thiol; (middle) $10 \mathrm{mM}$ octadecylthiol and $10 \mathrm{mM} \mathrm{BEMP;} \mathrm{(upper)} 100 \mathrm{mM}$

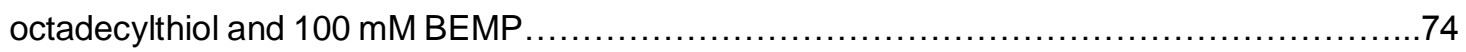

\section{CHAPTER 3}

Figure 1. Base and (thio)urea cocatalysts evaluated for ROP.

Figure 2. First order evolution of VL vs time for the TCC/MTBD catalyzed ring-opening polymerization of VL......

Figure 3. $M_{\mathrm{n}}$ (blue) and $M_{\mathrm{w}} / M_{\mathrm{n}}$ (orange) vs conversion for the TCC/MTBD catalyzed ringopening polymerization of VL. .97

Figure 4. GPC traces of the polymers resulting from the chain extension experiment of VL.....98

Figure 5. Approach to equilibrium evolution of [VL] vs time for the TCC/MTBD catalyzed ringopening polymerization of $\mathrm{VL}$ .98

Figure 6. $M_{\mathrm{n}}$ (blue) and $M_{\mathrm{w}} / M_{\mathrm{n}}$ (orange) vs conversion for the TCC/MTBD catalyzed ringopening polymerization of $\mathrm{VL}$ .99

Figure 7. GPC traces of the polymers resulting from the chain extension of PVL in acetone.....99 Figure 8. First order evolution of [L-LA] vs time for the TCC/Me ${ }_{6} \mathrm{TREN}$ catalyzed ring-opening polymerization. 100

Figure 9. Mn (blue) and Mw/Mn (orange) vs conversion for the TCC/Me6TREN catalyzed ringopening polymerization of $L-L A$. .100

Figure 10. Methine region of the methyl-decoupled ${ }^{1} \mathrm{H}$ NMR spectrum of PLLA obtained via TCC/Me6 TREN cocatalyzed ROP of L-LA $\left(500 \mathrm{MHz}, 25^{\circ}\right.$. 101

Figure 11. (upper) First order evolution of [VL] vs time for the di-CC/MTBD catalyzed ROP of VL. (lower). 102 
Figure 12. Downfield portion of the ${ }^{1} \mathrm{H}$ NMR spectra of TCC plus base $([T C C]=[$ base $]=5 \mathrm{mM})$ in acetone- $d_{6}$ 103

Figure 13. $M_{\mathrm{n}}$ (blue) and $M_{\mathrm{w}} / M_{\mathrm{n}}$ (orange) vs conversion for the TCC/BEMP catalyzed ringopening polymerization of $\mathrm{VL}$ 104

Figure 14. Titration binding curve for the CL/1-O binding in benzene- $d_{6}$. 105

Figure 15. Titration binding curve for the CL/1-S binding in benzene- $d_{6}$..... 105

Figure 16. ${ }^{1} \mathrm{H} \mathrm{NMR}\left(\mathrm{CD}_{3} \mathrm{OD}, 400 \mathrm{MHz}\right)$ spectrum of mono-CC 106

Figure 17. ${ }^{13} \mathrm{C} \mathrm{NMR}\left(\mathrm{CD}_{3} \mathrm{OD}, 100 \mathrm{MHz}\right)$ spectrum of mono-CC. 107

Figure 18. ${ }^{1} \mathrm{H} \mathrm{NMR}\left(\mathrm{CD}_{3} \mathrm{OD}, 400 \mathrm{MHz}\right)$ spectrum of di-CC 108

Figure 19. ${ }^{13} \mathrm{C} \mathrm{NMR}\left(\mathrm{CD}_{3} \mathrm{OD}, 100 \mathrm{MHz}\right)$ spectrum of di-CC. 109

Figure 20. First order evolution of $\mathrm{CL}$ vs time for the TCC/MTBD catalyzed ring-opening polymerization of $\mathrm{CL}$ 112

Figure 21. $M_{\mathrm{n}}$ (blue) and $M_{\mathrm{w}} / M_{\mathrm{n}}$ (orange) catalyzed ring-opening polymerization of $\mathrm{CL}$ 113

Figure 22. GPC traces of the polymers resulting from the chain extension experiment of VL..113 Figure 23. MALDI-TOF of the PLLA resulting from TCC/Me 6 TREN cocatalyzed ROP of Llactide. 114

\section{CHAPTER 4}

Figure 1. Catalytic systems employed by Hedrick and Waymouth. 118

Figure 2. Structures and identifying abbreviations for compounds mentioned. 118

Figure 3. First-order evolution of [VL] vs. time for the 3CTU/MTBD catalyzed ROP of VL.....119

Figure 4. Angles determined by Ingold.

Figure S1. Linear conversion of $M_{\mathrm{n}}$ versus percent conversion (circles) and $M_{\mathrm{w}} / M_{\mathrm{n}}$ versus conversion (squares) for the MTBD/5CTU catalyzed ROP of VL

Figure S2. Linear conversion of $M_{\mathrm{n}}$ versus percent conversion (circles) and $M_{\mathrm{w}} / M_{\mathrm{n}}$ versus percent conversion (squares) of the DBU/5CTU catalyzed ROP of VL 134

Figure S3. Types of Michaelis-Menten enzyme kinetics inhibition displayed as Linweaver-Burke plots. 135 
Figure S4. Linweaver-Burke plot of the MTBD (10 mM, squares; $100 \mathrm{mM}$, diamonds; $500 \mathrm{mM}$, circles) and 5CTU catalyzed polymerization of $\mathrm{VL}$ 136

Figure S5. First-order evolution of [VL] versus time for the MTBD/CyTU catalyzed ROP of VL. 136

Figure S6. First-order evolution of [VL] versus time for the MTBD/2CTU catalyzed ROP of VL. 137

Figure S7. First-order evolution of [VL] versus time for the MTBD/3CTU catalyzed ROP of VL. 137

Figure S8. First-order evolution of [VL] versus time for the MTBD/4CTU catalyzed ROP of VL. 138

Figure S9. Second-order evolution of [VL] versus time for the MTBD/5CTU catalyzed ROP of VL. 138

Figure S10. Second-order evolution of [VL] versus time for the MTBD/6CTU catalyzed ROP of VL. 139

Figure S11. Second-order evolution of [VL] versus time for the MTBD/12CTU catalyzed ROP of VL 139

Figure S12. Second-order evolution of [VL] versus time for the DBU/4CTU catalyzed ROP of VL. 140

Figure S13. Second-order evolution of [VL] versus time for the DBU/5CTU catalyzed ROP of VL. 140

Figure S14. Second-order evolution of [VL] versus time for the DBU/6CTU catalyzed ROP of VL. 141

Figure S15. Second-order evolution of [VL] versus time for the DBU/12CTU catalyzed ROP of VL. 141

Figure S16. Second-order evolution of [VL] versus time for the BEMP/2CTU catalyzed ROP of VL. 142

Figure S17. Second-order evolution of [VL] versus time for the BEMP/3CTU catalyzed ROP of VL. 142 
Figure S18. Second-order evolution of [VL] versus time for the BEMP/4CTU catalyzed ROP of VL. 143

Figure S19. Second-order evolution of [VL] versus time for the BEMP/5CTU catalyzed ROP of VL. 143

Figure S20. Second-order evolution of [VL] versus time for the BEMP/6CTU catalyzed ROP of VL. 144

Figure S21. Second-order evolution of [VL] versus time for the BEMP/12CTU catalyzed ROP of $\mathrm{VL}$. 144

Figure S22. $k_{i e}$ of the DBU/5CTU catalyzed ROP of VL. 146

Figure S23. Top) ${ }^{1} \mathrm{H}$ NMR of $2 \mathrm{CTU}$ in acetone- $d_{6}$. Bottom) ${ }^{13} \mathrm{C}$ NMR of $2 \mathrm{CTU}$ in acetone- $d_{6.147}$ Figure S24. Top) ${ }^{1} \mathrm{H}$ NMR of $4 \mathrm{CTU}$ in acetone- $d_{6}$. Bottom) ${ }^{13} \mathrm{C}$ NMR of $4 \mathrm{CTU}$ in acetone- $d_{6.148}$ Figure S25. Top) ${ }^{1} \mathrm{H}$ NMR of $5 \mathrm{CTU}$ in acetone- $d_{6}$. Bottom) ${ }^{13} \mathrm{C}$ NMR of $5 \mathrm{CTU}$ in acetone- $d_{6.149}$ Figure S26. Top) ${ }^{1} \mathrm{H}$ NMR of $6 \mathrm{CTU}$ in acetone- $d_{6}$. Bottom) ${ }^{13} \mathrm{C}$ NMR of $6 \mathrm{CTU}$ in acetone- $d_{6.150}$ Figure S27. Top) ${ }^{1} \mathrm{H}$ NMR of $12 \mathrm{CTU}$ in acetone- $d_{6}$. Bottom) ${ }^{13} \mathrm{C}$ NMR of $12 \mathrm{CTU}$ in acetone$d_{6} \ldots$ .151 


\section{LIST OF SCHEMES}

\section{CHAPTER 1}

Scheme 1. Synthesis of B-thiopropiolactone using B-mercapto propionic acid reacted with

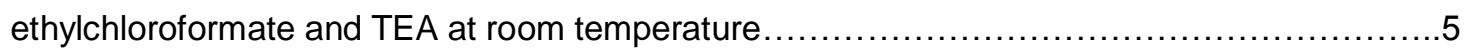

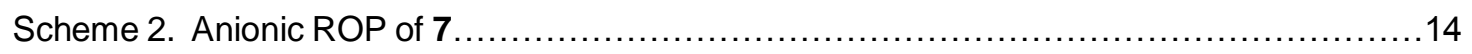

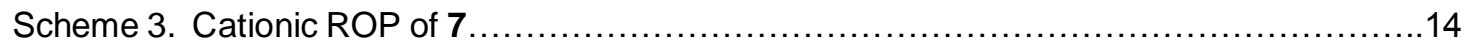

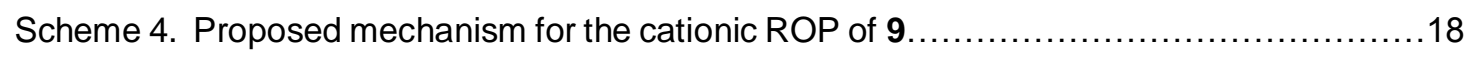

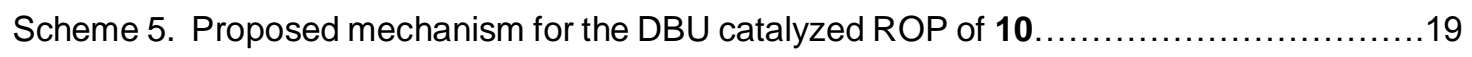

Scheme 6. Proposed mechanism for the cationic isomerization and polymerization of $11 \ldots \ldots .24$

Scheme 7. Proposed mechanism for the cationic isomerization and polymerization of $\mathbf{1 1}$ using

TfOEt or TfOH as catalysts supported by mechanistic studies.............................25

Scheme 8. Reaction conditions for the formation of a functionalized dithiocarbonate............26

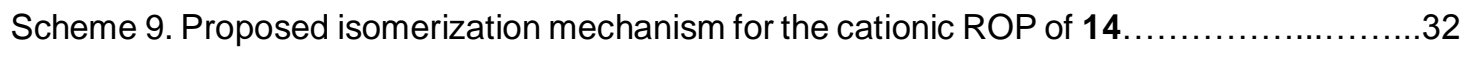

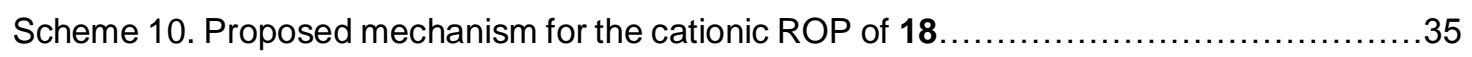

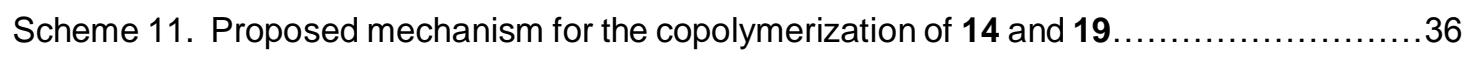

Scheme 12. Proposed isomerization mechanism for the cationic ROP of $22 \ldots \ldots \ldots \ldots \ldots \ldots . \ldots . \ldots . \ldots . \ldots$

\section{CHAPTER 2}

Scheme 1. Nucleophilic Mechanism for the ROP of $\mathrm{tCL}$ with DBU ...........................50

Scheme 2. Proposed Bifunctional Mechanism for the ROP of tCL by BEMP/1 Cocatalysts......53

\section{CHAPTER 3}

Scheme 1. Proposed mechanism for TCC/base cocatalyzed ROP

\section{CHAPTER 5}

Scheme 1. The homo-cross-metathesis reaction of stilbene produces one non-volatile product. .149

Scheme 2. Example Wittig reaction to synthesize stilbene 150 


\section{LIST OF EQUATIONS}

\section{CHAPTER 2}

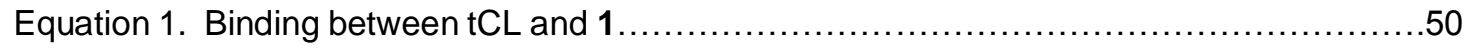

Scheme 2. Proposed Bifunctional Mechanism for the ROP of tCL by BEMP/1 Cocatalysts......53

CHAPTER 3

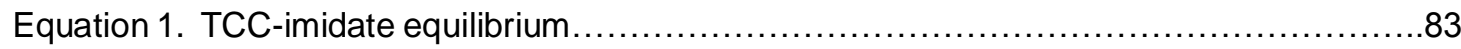




\section{CHAPTER 1}

Prepared for publication in Macromolecules.

Ring-Opening Polymerization of Thiol Containing Cyclic Carbonate and Lactone Monomers: A

Review

Timothy J. Bannin

Chemistry, University of Rhode Island, Kingston, RI USA 


\section{ABSTRACT}

Organocatalytic mediated ring-opening polymerizations (ROP) are robust and controllable pathways to well defined S-containing polyesters and polycarbonates. The following review attempts to bring together the myriad of work completed on the ROP of thio(esters) and thio(carbonates). The history entailing the ROP of thioesters and thiocarbonates is presented along with the challenges and strengths. 


\section{INTRODUCTION}

Polyesters and polycarbonates have become a highly sought-after source of renewable and biodegradable materials over the last 60 -70 years. Much research has been done in this area, however cyclic sulfur containing lactone and carbonate monomers have been virtually neglected. The reason is twofold; the monomers tend to be difficult and costly to make and obtaining a product that is well-controlled appears to be no easy feat. However, the advent of ring-opening polymerization techniques has started to change our outlook on the controlled polymerization of S-containing lactones and carbonates. S-containing polyesters/carbonates exhibit excellent mechanical and thermal properties. Poly(thioester)s and poly(thiocarbonate)s appear to be good candidates for anti-fouling/anti-bacterial coating, exhibit relatively high refractive indexes, and possible biodegradable properties. 


\section{THIOESTERS}

Fries and Mengel reported the first known synthesis of a thiolactone in 1912., ${ }^{1,2}$ When treating certain lactones with $\mathrm{P}_{4} \mathrm{~S}_{10}$ the authors found $\gamma$-thiovalerolactone (1, Figure 1) and $\gamma$ thionovalerolactone (2, Figure 1) were synthesized in low yields. However,
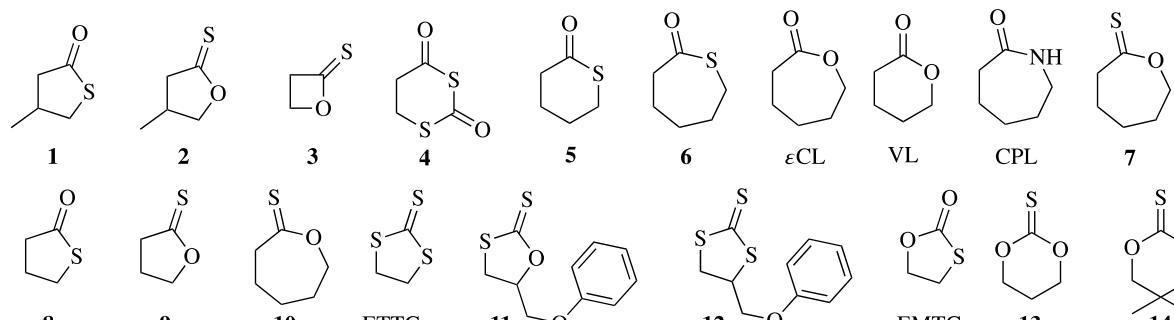

6

$\varepsilon \mathrm{CL}$

VL

CPL

7
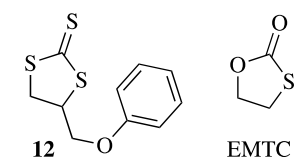

EMTC
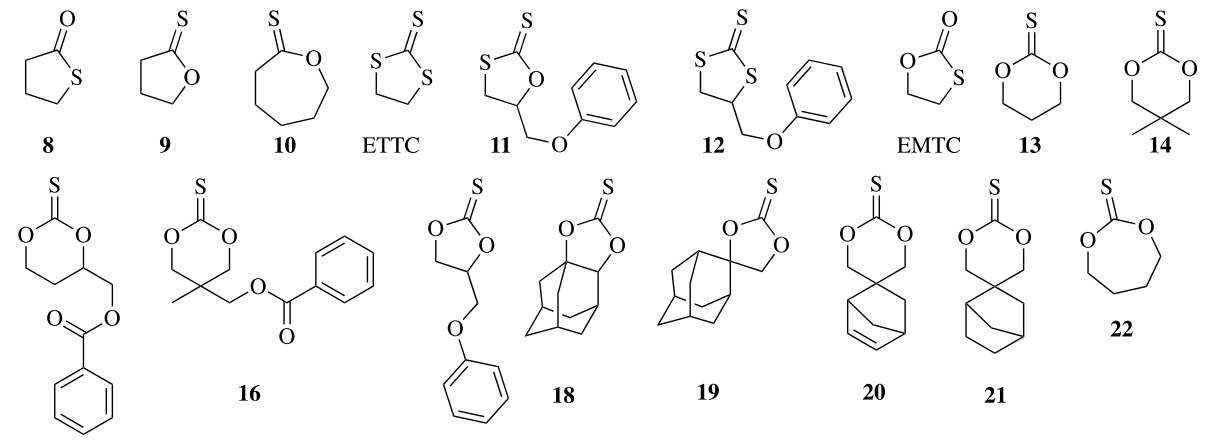

$9 \quad \mathbf{1 0} \quad$ ETTC
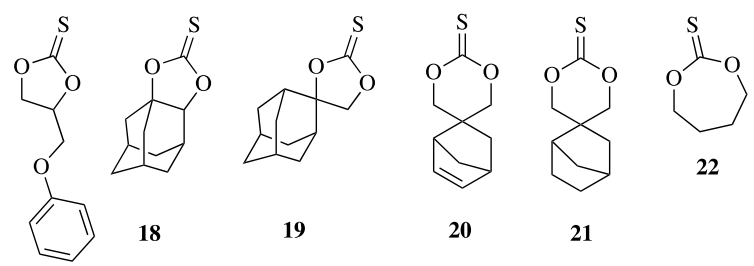

16

17

Figure 1. Monomers with abbreviations used in this paper.

because of the thermodynamic properties of the 5 -membered lactones no homopolymers have been made to date as far as the authors know; albeit not for a lack of trying. The four, six, and seven membered rings are thermodynamically favored to open; meaning these species make up the bulk of the research pertaining to the ROP of S-containing cyclic monomers, but it wasn't until 1958 that cyclic esters were looked at in closer detail.

B.F. Goodrich detailed the synthesis and properties of B-thiopropiolactone (3) in a British patent in $1958 .^{3}$ The patent describes a process where B-chloro (or bromo) propionyl chloride was mixed with sodium sulfide in order to incorporate the sulfur into the final product. Around the same time, a Russian research group led by Knunyants and Lin'kova described the preparation of several substituted B-thiolactones. ${ }^{4-11}$ Later, in 1965, the same Russian research group reported the synthesis of an unsubstituted ß-thiopropiolactone. ${ }^{12}$ Knunyants et al. combined hydrogen sulfide $\left(\mathrm{H}_{2} \mathrm{~S}\right)$ and trimethylamine (TEA) in order to incorporate the sulfide into the final 
product. ${ }^{10}$ These same authors suggested another synthetic approach utilizing B-mercapto

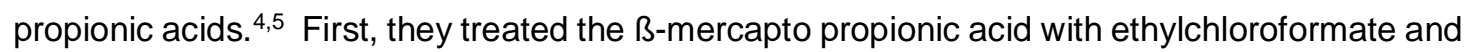
TEA at room temperature; of which yielded a mixed anhydride (Scheme 1). Being unstable, the mixed anhydride quickly cyclized releasing a primary alcohol and $\mathrm{CO}_{2}$ gas.

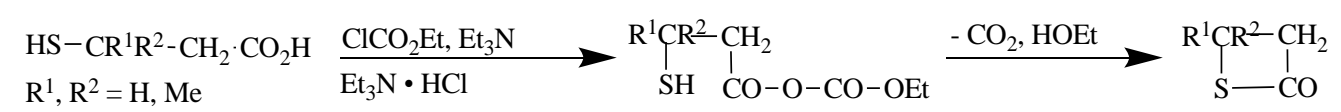

Scheme 1. Synthesis of B-thiopropiolactone using B-mercapto propionic acid reacted with ethylchloroformate and TEA at room temperature.

Although, B-thiolactones have been studied rather extensively, ${ }^{4-13}$ the polymerization of $\mathrm{B}$ thiopropiolactone was not. Several B-thiolactones were polymerized using water as an initiator and heating in a small sealed tube. The authors obtained low weight poly(ß-thiolactone)s, but did not report the exact molecular weights or Tm's.

Kricheldorf described a new synthetic approach to making 1,3-dithian-2,4-dione (4) via the cyclization reaction of $B$-xanthogene propionylchloride. ${ }^{14}$ Where the five-membered dithiolanedione (DTD) monomers are relatively easily formed, the six-membered conjugate tends to be much more difficult. These monomers require high temperatures and long reaction times, which lead to the decomposition and oligomerization of the monomer leading the authors to report a $16 \%$ yield from the few trials completed. However, the six-membered DTD polymerizes much more readily when tertiary amines are used as catalysts. When primary amines are used a stoichiometric reaction took place. When Kricheldorf applied TEA as the catalyst in the ROP of a six-membered DTD it yielded moderate weight poly(thioester)s. Remarkably, the poly(thioester)s exhibited higher than normal chemical and thermal stability.

The author wishes to inform the reader the majority of the rest of this section is dedicated to the ROP, properties, and comparisons to other lactones of both $\delta$-thiovalerolactone $(5)$ and $\varepsilon^{-}$ thiocaprolactone (6). Overberger and Weise (1968) reported the first ROP of $5,{ }^{15}$ but not the first synthesis. It appears the first synthesis was reported back in $1941^{16}$ with a few other groups reporting alternative approaches to the synthesis of monomer 5 over the next two decades. ${ }^{17-}$ 19 To synthesize monomer $\mathbf{5}, \delta$-mercaptovaleric acid was cyclized via several different 
approaches. One approach used thioacetic acid added to allylacetic acid followed by hydrolysis of the acetyl group making methyl acetate a prime leaving group. ${ }^{17}$ Another approach includes reacting $\delta$-chlorovaleric acid with sodium hydrosulfide $(\mathrm{NaSH})$ or a thiourea. ${ }^{18}$ BASF patented an approach that included reacting $\delta$-valerolactone $(\mathrm{VL})$ with hydrogen sulfide $\left(\mathrm{H}_{2} \mathrm{~S}\right)$ and a stoichiometric amount of a basic reagent like potassium carbonate $\left(\mathrm{K}_{2} \mathrm{CO}_{3}\right) .{ }^{19}$ Alternatively, unsubstituted thiol acids can be spontaneously cyclized when heated to remove water. ${ }^{15}$ Cyclization was also obtained by reaction of the unsubstituted lactone with phosphorus pentasulfide $\left(\mathrm{P}_{2} \mathrm{~S}_{5}\right)$. Consequently, 6 may be synthesized in two different approaches; by either cyclization of $\varepsilon$-mercaptohexanoic acid ${ }^{20-22}$ or reacting $\varepsilon \mathrm{CL}$ with $\mathrm{H}_{2} \mathrm{~S}$ and a basic catalyst. ${ }^{16}$

Overberger reported monomer $\mathbf{5}$ produces linear polymer when exposed to strong bases (potassium $t$-butoxide $(\mathrm{KOtBu}))$ and heat $\left(155^{\circ} \mathrm{C}\right)$ in the absence of water. ${ }^{15}$ However, the yield and intrinsic viscosities obtained were low. Further studies showed the monomer concentration at equilibrium to be high; which accounts for the low yield and molecular weights obtained. The polymer created from the ROP of 6 was obtained via several different methods. Notably, one involves a catalytic amount of $\mathrm{KOtBu}$ in an inert atmosphere with heat applied $\left(150^{\circ} \mathrm{C}\right)$. However, the reaction will proceed rapidly and uncontrolled at room temperature if $n$-butyllithium is utilized as the catalyst. Overberger applied other bases of varying strength to the ROP of 6 and ultimately showed strong bases served as the best catalysts. Cationic catalysts yield low weight, colored polymers. Whereas the anionic catalyst, triethylaluminum, produced a robust polymer in high yield. Overberger suggests the process takes place via anionic ROP where the polymerization is initiated by nucleophilic attack at the carbonyl of the monomer. Propagation occurs at the sulfur anion and termination takes place in the presence of water or a mild organic acid, i.e. benzoic acid. This group also successfully polymerized both $\mathbf{5}$ and $\mathbf{6}$ in bulk. The bulk polymerization of 5 resulted in low molecular weight polymers in low quantities. However, when 6 was polymerized in bulk the results were much different; high yield and high molecular weight polymer was produced. It should be added that one other group patented three different approaches to the synthesis and polymerization of $6 .{ }^{23-25}$ Fritze outlines a synthesis where $\varepsilon \mathrm{CL}$ is heated in the presence carbon disulfide $\left(\mathrm{CS}_{2}\right)$ and sodium hydroxide $(\mathrm{NaOH})$ at $200^{\circ} \mathrm{C}$, of 
which yielded low weight poly(thiocaprolactone)s. Via thermal depolymerization, the monomeric 6 was synthesized and then homopolymerized and copolymerized with $\varepsilon C L$.

Several other research groups worked with $\mathbf{6}$ as well. ${ }^{26-28}$ Notably, Matzner et al. used $\mathbf{6}$ as an initiator in the ROP of caprolactam (CLa) with success. ${ }^{26}$ Using sodium hydride (NaH) as a catalyst in the bulk polymerization of CPL, the authors obtained polymers with high molecular weights and fast reaction times ( $\sim 5 \mathrm{~min}$.$) . Another research group synthesized a myriad of$ cyclic lactones by reacting $\omega$-halo acid chlorides of various sizes (Table 1) with benzyltriethylammonium tetrathiomolybdate as a catalyst and solvent $\left(\mathrm{CHCl}_{3}\right.$ or $\left.\mathrm{CH}_{3} \mathrm{CN}\right)$ in moderate to good yields $(11-77 \%) .{ }^{27}$ Bhar and Chandrasekaran paved the way to an easy one pot synthesis of macrocyclic lactones through their method. ${ }^{27}$

It was not until the last two decades that the organocatalytic ROP of cyclic lactones was studied. The IBM Almaden Research Institute in San Jose, CA revolutionized the way we approach ringopening polymerizations. ${ }^{29}$ Until this work was published, most ROP procedures for Scontaining monomers included very strong bases, uncontrolled results, and low yields. However, organocatalytic living ROP provides a mild yet robust, very controllable, high yielding method capable of producing a myriad of polymers. Utilizing dimethylaminopyridine (DMAP) and pyrrolidinopyridine (PPY) as catalysts Nederberg et al. successfully conducted the organocatalytic living ROP of lactide. Throughout this work, the authors were able to control polymer chain length by tailoring the monomer-to-initiator $\left([\mathrm{M}]_{0} /[]_{0}\right)$ feed ratios. Notably, it should be mentioned that even at conversions up to $100 \%$ (by ${ }^{1} \mathrm{H}$ NMR) the molecular weight distribution $(\boxminus)$ remained low even at long reaction times; implying no substantial transesterification of the polymer backbone taking place.

\begin{tabular}{cccccc} 
entry & substrate & product & temp $\left({ }^{\circ} \mathrm{C}\right)$ & time $(\mathrm{h})$ & yield $(\%)$ \\
\hline $\mathrm{CCOCl}$ & & 0 & 4 & $30^{\mathrm{a}}$
\end{tabular}


2<smiles>O=C(Cl)CCCBr</smiles><smiles>O=C1CCCS1</smiles>

3<smiles>O=C(Cl)CCCCBr</smiles>

4<smiles>O=C(Cl)CCCCCCBr</smiles>
$\mathrm{COCl}$<smiles>O=C1CCCCCS1</smiles>

Dimer

5 $\mathrm{BrCH}_{2}\left(\mathrm{CH}_{2}\right)_{9} \mathrm{COCl}$

6 $\mathrm{BrCH}_{2}\left(\mathrm{CH}_{2}\right)_{9} \mathrm{COCl}$<smiles>O=C1CCCCS1</smiles>
$12+$
0

7 $77^{a}$ $13^{b}$

25 $16^{b}$ 0 24 $31^{b}$ 25 24

Dimer

$65^{a}$

24

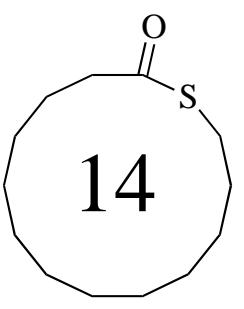

$11^{b}$ $24^{b}$

Table 1. Reaction of $\omega$-halo acid chlorides with tetrathiomolybdate in solution. Solvent: ${ }^{a} \mathrm{CHCl}_{3}$. ${ }^{b} \mathrm{CH}_{3} \mathrm{CN}$.

Within the last decade the application of organocatalytic ROP has been directed toward Scontaining monomers. Our group reported the organocatalytic ROP of $6 .{ }^{22}$ Strong imine bases such as 1,5,7-triazabicyclo[4.4.0]dec-5-ene (TBD) and octadecylthiol as an initiator in $\mathrm{CDCl}_{3}$ effected the organocatalytic ROP of 6 at room temperature. This approach yielded poly(thioester)s readily and controllably.

However, if the reaction was left to reach equilibrium, transesterification does take place as TBD is a known transesterification agent. ${ }^{29}$ Adding a thiourea (TU) co-catalyst into the polymerization increased the livingness of the system, i.e. displayed a linear evolution of molecular weight as 
percent conversion increased. A myriad of bases was applied to the organocatalytic ROP of 6 , including DMAP, tris[2-(dimethylamino)ethyl]amine (Me6TREN), 2-tert-butylamino-2diethylamino-1,3-dimethylperhydro-1,3,2-diazaphosphorine $\quad$ (BEMP), $\quad$ 1,8diazabicyclo[5.4.0]undec-7-ene (DBU), and 7-methyl-1,5,7-triazabicyclo[4.4.0]dec-5-ene (MTBD). This base screen showed only strong nucleophilic bases effect the organocatalyzed ROP of $\mathbf{6}$, but the $Đ$ of the polymer was high for a living polymerization when compared to polyesters. ${ }^{22}$ The increased nucleophilicity of thiols compared to alcohols may contribute to the increased $Đ$. Notably the authors experimentally determined the thermodynamic data of 6 (Figure 2) via Van't Hoff analysis and found that 6 is more energetically akin to CPL than $\varepsilon C L$ and VL because of the lack of ceiling temperature.

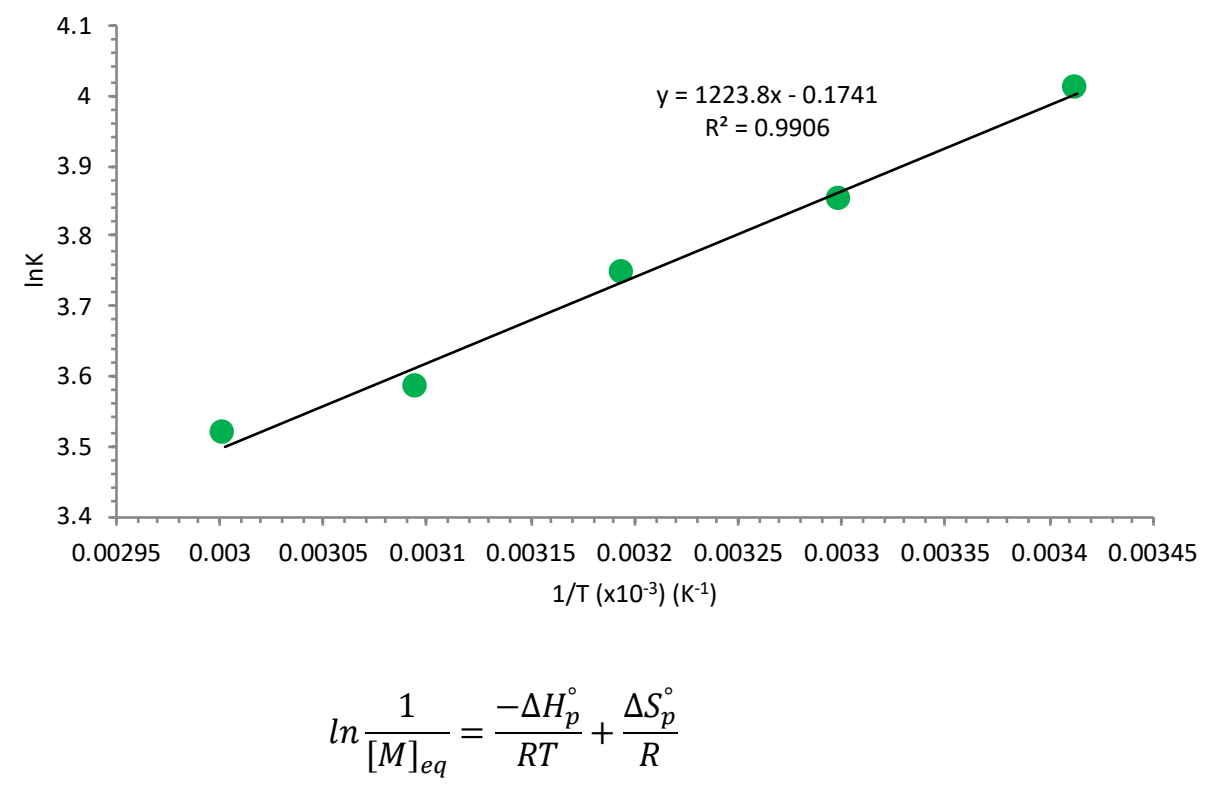

Figure 2. Van't Hoff plot for the TBD catalyzed ROP of $\varepsilon$-tCL.

Much research has been conducted into thiolactones and the polythioesters synthesized from them; however, the thionolactones have not been as frequently reported. To the author's best knowledge, the first two reported synthetic routes to a thionolactone were reported by Kaloustian and coworkers. ${ }^{30,31}$ Kaloustain and Nader first synthesized the $\mathrm{N}, \mathrm{N}$-dimethyliminolatonium salt by treating the respective lactone with a tertiary amine (like dimethylamine) and thionylchloride 
$\left(\mathrm{SOCl}_{2}\right)$ resulting in the linear amide product. This was then treated with silver tetrafluoroborate $\left(\mathrm{AgBF}_{4}\right)$ of which produced the $\mathrm{N}, \mathrm{N}$-dimethyliminolactonium fluoroborate salt. The salt was then reacted with $\mathrm{NaSH}$ in acetone at $-78^{\circ} \mathrm{C}$ and then with acetylchloride $(\mathrm{AcCl})$ in pyridine to produce the respective thionolactone in good to moderate yields. Soon thereafter, Kaloustian and Khouri reported a more convenient synthetic route to thionolactones from lactones. ${ }^{31}$ This new method involved treating the respective cyclic lactone with Meerwein's salts $\left(\mathrm{R}_{3} \mathrm{O}^{+} \mathrm{BF}_{4^{-}}, \mathrm{R}=\mathrm{Me}\right.$, or Et) to yield the alkylated lactonium salt. Next, the lactonium salt was treated with anhydrous sodium hydrosulfide at $0^{\circ} \mathrm{C}$ to produce the respective thionolactone moderate to good yields. The authors noted as the ring size increased so did the by-products produced from the synthesis. They also noted if the reaction is carried out at $-78^{\circ} \mathrm{C}$ the byproducts are severely limited. A number of substituted and unsubstituted five-, six-, and seven membered thionolactones were successfully synthesized in this manner. However, the authors did not investigate the ROP of these entities.

Following in the footsteps of Kaloustian and coworkers, Nicolau et al. synthesized a number of substituted and unsubstituted seven-, nine-, ten-, and seventeen- membered thionolactones; albeit through several different Lawesson's reagents (LR). ${ }^{32-34}$ Nicolau et al. did not investigate the ROP of their monomers either, but instead transformed them into cyclic ethers. Arguably the easiest and most utilized thionation reaction was proposed ${ }^{35-37}$ and patented ${ }^{38}$ by Curphey out of Dartmouth. Curphey treated the respective lactone or ester (Figure 3) with dimeric 


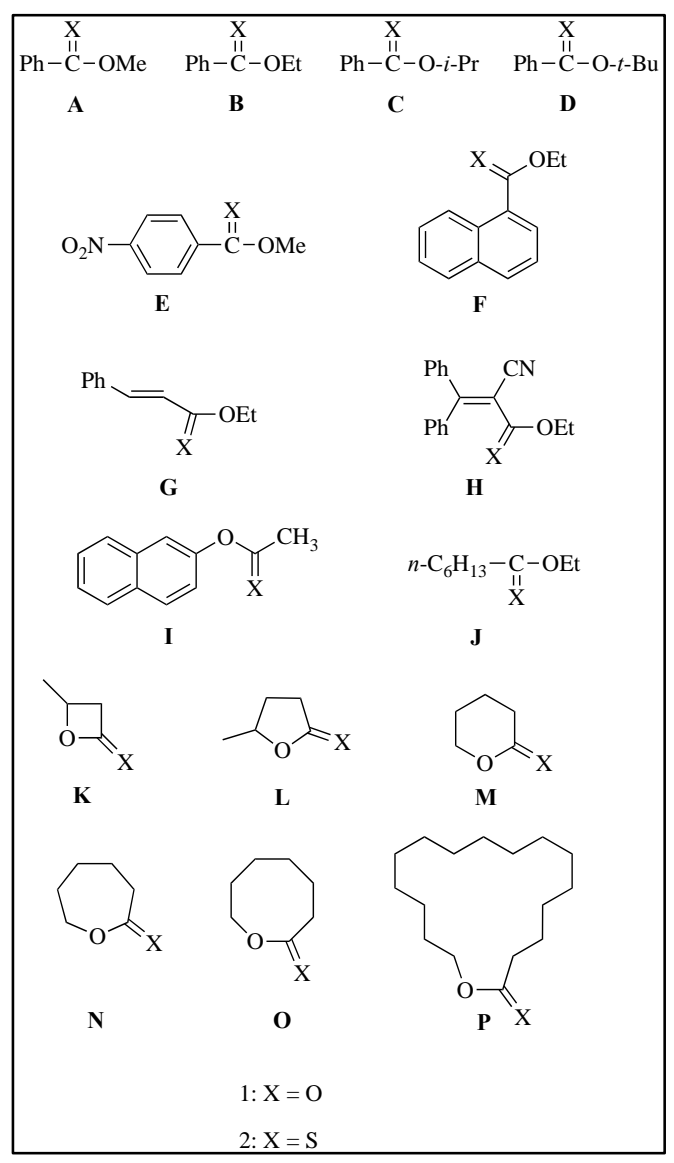

Figure 3. Esters, thionoesters, lactones, and thionolactones.

phosphorus pentasulfide $\left(\mathrm{P}_{4} \mathrm{~S}_{10}\right)$ and hexamethyldisiloxane (HMDO). ${ }^{37}$ This new method increased the yield, eased the purification (hydrolytic workup or silica gel purification) of the thionolactone or thionoester, and decreased the reaction time when compared to the traditional LR's, Table 2. However, Curphey did not inquire into the ROP of the thionolactones synthesized. 


\begin{tabular}{|c|c|c|c|c|c|c|}
\hline \multirow{2}{*}{ entry } & \multirow{2}{*}{ ester/lactone } & \multirow{2}{*}{ reagent } & \multirow{2}{*}{ solvent } & \multirow{2}{*}{ time $(h)^{b}$} & \multicolumn{2}{|c|}{ yield (\%) } \\
\hline & & & & & HPLC & Isolated $^{c}$ \\
\hline $1^{d}$ & $A^{1}$ & $\mathrm{P}_{4} \mathrm{~S}_{10} / \mathrm{HDMO}$ & xylene & 10 & 92 & 79 \\
\hline 2 & $A^{1}$ & LR & xylene & 8 & 92 & \\
\hline $3^{d}$ & $A^{1}$ & $\mathrm{P}_{4} \mathrm{~S}_{10}$ & xylene & 18 & 67 & \\
\hline $4^{d}$ & $\mathrm{~B}^{1}$ & $\mathrm{P}_{4} \mathrm{~S}_{10} / \mathrm{HDMO}$ & xylene & 8 & 81 & 73 \\
\hline 5 & $\mathrm{~B}^{1}$ & LR & xylene & 8 & 81 & \\
\hline $6^{d}$ & $\mathrm{~B}^{1}$ & $\mathrm{P}_{4} \mathrm{~S}_{10}$ & xylene & 8 & 61 & \\
\hline $7^{d}$ & $C^{1}$ & $\mathrm{P}_{4} \mathrm{~S}_{10} / \mathrm{HDMO}$ & xylene & 8 & 95 & 83 \\
\hline 8 & $C^{1}$ & LR & xylene & 8 & 92 & \\
\hline 9 & $E^{1}$ & $\mathrm{P}_{4} \mathrm{~S}_{10} / \mathrm{HDMO}$ & xylene & 14 & 28 & 21 \\
\hline 10 & $E^{1}$ & LR & xylene & 17 & 4 & \\
\hline 11 & $\mathrm{~F}^{1}$ & $\mathrm{P}_{4} \mathrm{~S}_{10} / \mathrm{HDMO}$ & xylene & 10 & 91 & 87 \\
\hline 12 & $\mathrm{~F}^{1}$ & LR & xylene & 12 & 83 & \\
\hline 13 & $\mathrm{G}^{1}$ & $\mathrm{P}_{4} \mathrm{~S}_{10} / \mathrm{HDMO}$ & PhMe & 4 & 75 & 72 \\
\hline 14 & $\mathrm{G}^{1}$ & LR & PhMe & 6 & 70 & \\
\hline 15 & $\mathrm{H}^{1}$ & $\mathrm{P}_{4} \mathrm{~S}_{10} / \mathrm{HDMO}$ & xylene & 16 & 51 & 42 \\
\hline 16 & $\mathrm{H}^{1}$ & LR & xylene & 30 & 59 & \\
\hline 17 & $\mathrm{I}^{1}$ & $\mathrm{P}_{4} \mathrm{~S}_{10} / \mathrm{HDMO}$ & PhEt & 17 & 41 & 30 \\
\hline 18 & $I^{1}$ & LR & PhEt & 15 & 40 & \\
\hline 19 & $J^{1}$ & $\mathrm{P}_{4} \mathrm{~S}_{10} / \mathrm{HDMO}$ & xylene & 4 & 87 & 75 \\
\hline 20 & $\mathrm{~J}^{1}$ & LR & xylene & 8 & 76 & \\
\hline 21 & $\mathrm{~J}^{1}$ & $\mathrm{P}_{4} \mathrm{~S}_{10}$ & xylene & 4 & 35 & \\
\hline 22 & $\mathrm{~L}^{1}$ & $\mathrm{P}_{4} \mathrm{~S}_{10} / \mathrm{HDMO}$ & $\mathrm{MeCN}$ & 1.5 & 87 & 78 \\
\hline 23 & $L^{1}$ & LR & PhMe & 3 & 85 & \\
\hline 24 & $\mathrm{M}^{1}$ & $\mathrm{P}_{4} \mathrm{~S}_{10} / \mathrm{HDMO}$ & $\mathrm{MeCN}$ & 0.75 & 82 & 65 \\
\hline 25 & $M^{1}$ & LR & $\mathrm{MeCN}$ & 4 & 71 & \\
\hline 26 & $\mathrm{~N}^{1}$ & $\mathrm{P}_{4} \mathrm{~S}_{10} / \mathrm{HDMO}$ & $\mathrm{MeCN}$ & 0.4 & 82 & 77 \\
\hline
\end{tabular}




$\begin{array}{lcccccc}27 & \mathrm{~N}^{1} & \mathrm{LR} & \mathrm{PhMe} & 1 & 73 & \\ 28 & \mathrm{~N}^{1} & \mathrm{P}_{4} \mathrm{~S}_{10} & \mathrm{MeCN} & 0.25 & 31 & \\ 29 & \mathrm{O}^{1} & \mathrm{P}_{4} \mathrm{~S}_{10} / \mathrm{HDMO} & \mathrm{MeCN} & 0.5 & 77 & 73 \\ 30 & \mathrm{O}^{1} & \mathrm{LR} & \mathrm{PhMe} & 1 & 58 & \\ 32 & \mathrm{O}^{1} & \mathrm{P}_{4} \mathrm{~S}_{10} & \mathrm{MeCN} & 0.3 & 35 & \\ 33 & \mathrm{P}^{1} & \mathrm{P}_{4} \mathrm{~S}_{10} / \mathrm{HDMO} & \text { xylene } & 4 & 87 & 86 \\ 34 & \mathrm{P}^{1} & \mathrm{LR} & \text { xylene } & 5 & 84 & \end{array}$

Table 2. Thionation of esters ${ }^{a}$ and lactones ${ }^{a}$. ${ }^{a}$ Reactions were run at reflux in the indicated solvent $\left(1 \mathrm{~mL} / \mathrm{mmol}\right.$ of ester/lactone). $\mathrm{P}_{4} \mathrm{~S}_{10}=0.25 \mathrm{mmol}$ per $\mathrm{mmol}$ of ester/lactone. $\mathrm{HMDO}=$ $1.67 \mathrm{mmol}$ per $\mathrm{mmol}$ of ester/lactone. For stoichiometry with respect to LR, see the Experimental. ${ }^{37} \quad b$ Time when the yield of thionation product was judged to have reached a maximum. ${ }^{c}$ Yield of isolated and purified material. ${ }^{d} \mathrm{P}_{4} \mathrm{~S}_{10}=0.33 \mathrm{mmol}$ per $\mathrm{mmol}$ of ester/lactone.

One alternative novel synthesis of thionolactones was completed by Filippi et al. ${ }^{39}$ Filippi and co-workers utilized LR $\mathrm{LR}^{33,34}$ in the presence of HDMO to perform a single sulfurization of a lactone to yield the respective thionolactone utilizing microwave support. In fact, the authors succeed in synthesizing several functionalized 5-membered $\gamma$-thionolactones, in excellent to moderate yields and extremely fast reaction times, Table 3 . They even applied the procedure to a 6 - and 7-membered cyclic lactone with moderate results.

The Endo laboratory appears to have conducted the first ROP of a thionolactone. Sanda et al. conducted extensive studies on $\varepsilon$-thionocaprolactone (7); applying both the anionic and cationic ROP. ${ }^{40,41}$ Poly(thionoester)s were obtained exclusively when lithium alkyls, lithium $t$-butoxide, and Grignard reagents were applied. However, a copolymer that contained mostly thiol ester groups was obtained when potassium-t-butoxide was applied. The authors did not address whether the copolymer was a block copolymer or random sequence. A mechanism was proposed for the anionic formation of the thionoester and thiolester groups and are shown in Scheme 2. The authors achieved both high molecular weights and high reaction rates when 

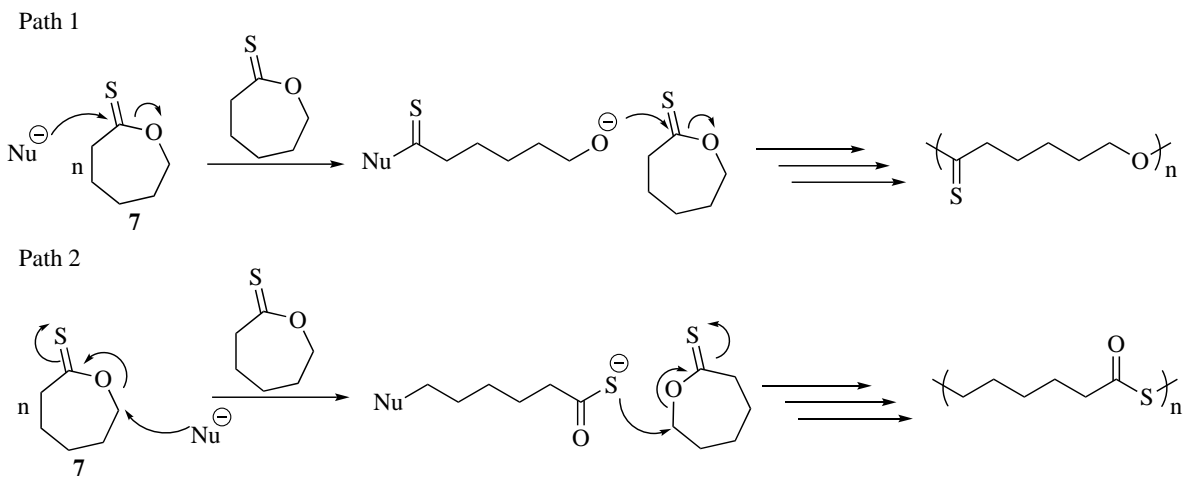

Scheme 2. Anionic ROP of 7.

the temperatures were elevated $\left(100^{\circ} \mathrm{C}\right)$ as seen in Table 4 . However, the authors discovered that only poly(thiolester)s were formed exclusively when cationic ROP conditions were applied to 7, Scheme 3; implying the polymerization took place via an isomerization reaction. Reaction rates and molecular weights increased with increased temperature between the temperatures tested $\left(-78-28^{\circ} \mathrm{C}\right)$, Table 5 . The authors also found that $M_{n}$ increased with solvent polarity and M/l. The authors suggested a plausible reaction scheme, Scheme 3.

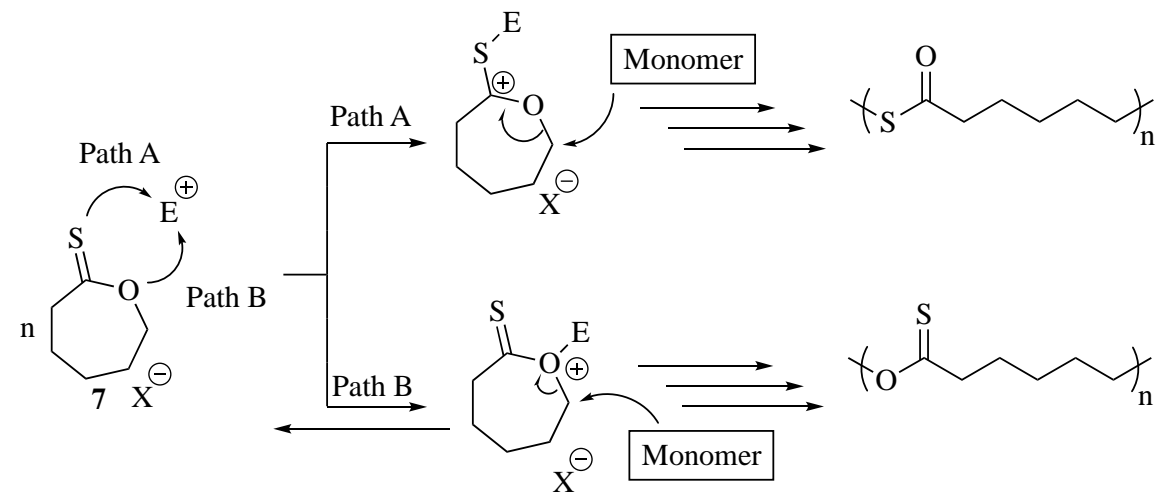

Scheme 3. Cationic ROP of 7. 


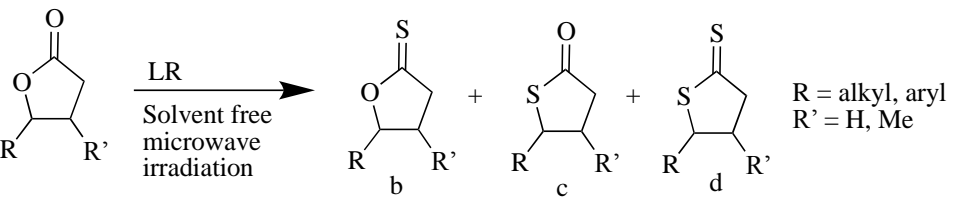

\begin{tabular}{|c|c|c|c|c|c|c|}
\hline entry & substrate & $\begin{array}{l}\text { reaction cond. }{ }^{a} \\
\text { (eq) }\end{array}$ & $\begin{array}{l}\text { time }^{b} \\
(\mathrm{~s})\end{array}$ & conv. ${ }^{c}(\%)$ & $\begin{array}{c}\text { selectivity }^{\circ} \\
(\%) \\
\text { b/c/d }\end{array}$ & $\begin{array}{r}\text { yield }^{e} \\
(\%)\end{array}$ \\
\hline 1 & & $\operatorname{LR}(0.5)$ & $4 \times 30$ & 80 & $87 / 0 / 13$ & $\begin{array}{l}66(b) \\
9(d)\end{array}$ \\
\hline 2 & & $\begin{array}{l}\mathrm{P}_{4} \mathrm{~S}_{10}(0.25) / \\
\mathrm{HDMO}(1.67)\end{array}$ & $5 \times 30$ & 94 & $86 / 0 / 14$ & $\begin{array}{l}74(b) \\
9(d)\end{array}$ \\
\hline 3 & & $\begin{array}{c}\text { LR (0.5)/ HDMO } \\
(0.5)\end{array}$ & $4 \times 30$ & 85 & $99 / 0 / 1$ & 76 \\
\hline 4 & & procedure $\mathrm{A}^{f}$ & $5 \times 30$ & 91 & 99/0/1 & 85 \\
\hline 5 & & procedure $\mathrm{A}$ & $3 \times 30$ & 94 & $95 / 0 / 5$ & 80 \\
\hline 6 & & procedure $\mathrm{A}$ & $3 \times 30$ & 95 & $99 / 0 / 1$ & 92 \\
\hline 7 & & procedure A & $4 \times 30$ & 98 & $95 / 0 / 5$ & 88 \\
\hline 8 & & procedure $\mathrm{A}$ & $5 \times 30$ & 95 & 99/0/1 & 90 \\
\hline 9 & & procedure $\mathrm{A}$ & $5 \times 30$ & 91 & $99 / 0 / 1$ & 89 \\
\hline 10 & & procedure $\mathrm{A}$ & $5 \times 30$ & 99 & $98 / 1 / 1$ & 96 \\
\hline 11 & & $\begin{array}{l}\text { procedure A/ } \\
\text { multi-gram synth }\end{array}$ & $2 \times 30$ & 93 & $96 / 0 / 4$ & 88 \\
\hline
\end{tabular}




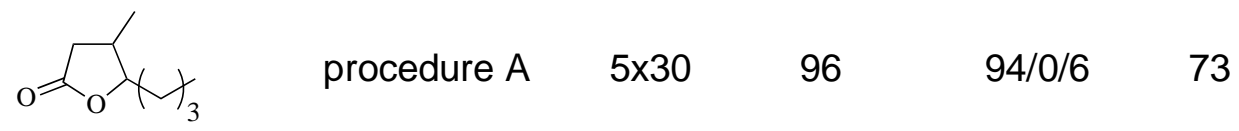<smiles>CC1CCCC(=O)O1</smiles>

Table 3. Synthesis of thionolactones (b) and dithiolactones (d) under microwave irradiation. ${ }^{a}$ For conditions see citation \#39. ${ }^{b}$ Number if irradiations for a given time; $3 \times 30$ indicates 3 irradiations of 30 seconds each. ${ }^{c}$ Determined by GC and calculated from hexadecane as internal standard. ${ }^{d}$ Determined by GC. ${ }^{e}$ Isolated yield. ${ }^{f}$ Procedure A: LR $=\mathrm{HDMO}=0.75$ eq. ${ }^{e}$ Consumption of the initial lactone $\neq$ conversion.

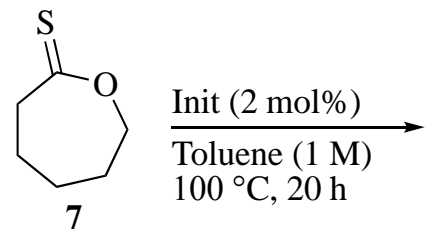<smiles>CSC(=O)CCCCC(C)(C)OCCCCCC(C)=S</smiles>

$\begin{array}{llccccc}\text { entry } & \text { initiator } & \text { conv. }^{b}(\%) & \text { yield }^{c}(\%) & M_{\mathrm{n}}{ }^{c} & M_{\mathrm{w}} / M_{\mathrm{n}}{ }^{d} & \text { unit ratio } \\ 1 & \text { MeLi } & 100 & 77 & 19,000 & 2.67 & \text { A:B } \\ 2 & n \text {-BuLi } & 100 & 65 & 8,200 & 1.75 & 100: 0 \\ 3 & \text { sec-BuLi } & 100 & 65 & 8,500 & 1.55 & 100: 0 \\ 4 & t-\mathrm{BuLi} & 100 & 58 & 8,300 & 1.53 & 100: 0\end{array}$




$\begin{array}{lllllll}5 & \text { PhLi } & 100 & 58 & 10,000 & 1.81 & 100: 0 \\ 6 & \text { MeMgCl } & 100 & 58 & 10,500 & 2.05 & 100: 0 \\ 7 & t \text {-BuMgCl } & 100 & 48 & 9,200 & 1.69 & 100: 0 \\ 8 & t \text {-BuOLi } & 98 & 52 & 9,200 & 1.78 & 100: 0 \\ 9 & t \text {-BuOK } & 30 & 17 & 3,800 & 1.54 & 37: 63 \\ 10 & \text { DBU } & 25 & 10 & 4,500 & 1.54 & 11: 89\end{array}$

Table 4. ${ }^{a}$ Anionic ROP of 7. ${ }^{a}$ Conditions: initiator $=2 \mathrm{~mol} \% ;[\mathrm{M}]_{0}=1 \mathrm{M}$ in toluene; $100 \stackrel{\circ}{\circ}$; and $20 \mathrm{~h} .{ }^{b}$ Determined by ${ }^{1} \mathrm{H}$ NMR $(400 \mathrm{MHz}) .{ }^{c} n$-Hexane-insoluble part. ${ }^{d}$ Estimated by GPC on the basis of polystyrene standards eluted by THF.

\begin{tabular}{|c|c|c|c|c|c|c|c|c|}
\hline entry & {$[\mathrm{M}] \mathrm{o} /[\mathrm{I}] \mathrm{o}$} & solvent & [M] & $\begin{array}{l}\text { temp } \\
\left({ }^{\circ} \mathrm{C}\right)\end{array}$ & $\begin{array}{c}\text { conv. }^{b} \\
(\%)\end{array}$ & $\begin{array}{c}\text { yield }^{c} \\
(\%)\end{array}$ & $M_{n}{ }^{d}$ & $M_{\mathrm{w}} / M_{\mathrm{n}}{ }^{d}$ \\
\hline 1 & 100 & $\mathrm{CH}_{3} \mathrm{NO}_{2}$ & 1.0 & 28 & 99 & 80 & 12,900 & 1.88 \\
\hline 2 & 100 & $\mathrm{CH}_{2} \mathrm{Cl}_{2}$ & 1.0 & 28 & 98 & 88 & 57,000 & 1.95 \\
\hline 3 & 100 & $\mathrm{PhCl}$ & 1.0 & 28 & 96 & 88 & 49,800 & 2.36 \\
\hline 4 & 100 & $\mathrm{CHCl}_{3}$ & 1.0 & 28 & 90 & 75 & 31,000 & 2.23 \\
\hline 5 & 100 & $\mathrm{PhH}$ & 1.0 & 28 & 97 & 77 & 24,200 & 2.81 \\
\hline 6 & 50 & $\mathrm{CH}_{2} \mathrm{Cl}_{2}$ & 1.0 & 28 & 100 & 80 & 50,000 & 2.10 \\
\hline 7 & 20 & $\mathrm{CH}_{2} \mathrm{Cl}_{2}$ & 1.0 & 28 & 100 & 76 & 48,000 & 2.17 \\
\hline 8 & 100 & $\mathrm{CH}_{2} \mathrm{Cl}_{2}$ & 1.0 & -78 & 0 & $--e$ & $--e$ & $--^{e}$ \\
\hline 9 & 100 & $\mathrm{CH}_{2} \mathrm{Cl}_{2}$ & 1.0 & -48 & 0 & $--^{e}$ & $--e$ & $--e$ \\
\hline 10 & 100 & $\mathrm{CH}_{2} \mathrm{Cl}_{2}$ & 1.0 & -30 & 0 & $-e^{e}$ & $--e$ & $--e$ \\
\hline 11 & 100 & $\mathrm{CH}_{2} \mathrm{Cl}_{2}$ & 1.0 & -15 & 2 & $--e$ & $--e$ & $--e$ \\
\hline 12 & 100 & $\mathrm{CH}_{2} \mathrm{Cl}_{2}$ & 1.0 & 0 & 51 & 22 & 8,600 & 1.60 \\
\hline 13 & 100 & $\mathrm{CH}_{2} \mathrm{Cl}_{2}$ & 1.0 & 10 & 70 & 49 & 20,600 & 1.72 \\
\hline 14 & 100 & $\mathrm{CH}_{2} \mathrm{Cl}_{2}$ & 0.1 & 28 & 23 & 10 & 9,000 & 1.58 \\
\hline 15 & 100 & $\mathrm{CH}_{2} \mathrm{Cl}_{2}$ & 0.5 & 28 & 80 & 73 & 48,000 & 2.85 \\
\hline
\end{tabular}


Table 5. Cationic polymerization of 7 initiated with $\mathrm{BF}_{3} \cdot \mathrm{OEt}_{2}{ }^{a}{ }^{a}$ Polymerization time $=5 \mathrm{~h} .{ }^{b}$ Determined by ${ }^{1} \mathrm{H}$ NMR (400 MHz). ${ }^{c}$ Methanol-insoluble part. ${ }^{d}$ Estimated by GPC based on polystyrene standards eluted by THF. ${ }^{e}$ Not determined.

The Endo lab also compared the ROP of $\gamma$-thiobutyrolactone (8) and $\gamma$-thionobutyrolactone (9). ${ }^{42}$ Monomer 9 was synthesized using the LR method. ${ }^{39}$ Cationic initiators, lanthanide triflates, were utilized as the initiators on their study into the ROP of 9, Table 6. Initially, the bulk polymerization was attempted of 8 at $100^{\circ} \mathrm{C}$.

$\begin{array}{lcccc}\text { entry } & \text { initiator } & \text { conv. }^{b}(\%) & \text { yield }^{c}(\%) & M_{\mathrm{n}}\left(\mathrm{M}_{\mathrm{w}} / \mathrm{Mn}_{\mathrm{n}}\right)^{d} \\ 1 & \mathrm{Sc}(\mathrm{OTf})_{3} & 78 & 50 & 3,400(2.2) \\ 2 & \mathrm{Y}(\mathrm{OTf})_{3} & 79 & 42 & 4,500(2.7) \\ 3 & \mathrm{Yb}(\mathrm{OTf})_{3} & 84 & 45 & 3,600(2.9) \\ 4 & \mathrm{La}(\mathrm{OTf})_{3} & 53 & 30 & 6.300(2.2)\end{array}$

Table 6. ROP of 9.

However, the authors reported that this polymerization did not take place; presumably because of the thermodynamic properties. The ROP of $\mathbf{9}$ was conducted in bulk utilizing scandium

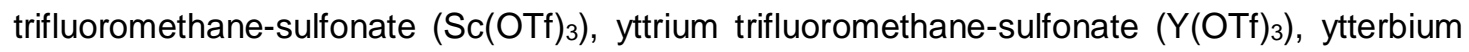
trifluoromethane-sulfonate $\left(\mathrm{Yb}(\mathrm{OTf})_{3}\right)$, and lanthanum trifluoromethane-sulfonate $\left(\mathrm{La}(\mathrm{OTf})_{3}\right)_{\text {. }}$. The authors obtained moderate conversions, molecular weights, and broad $Đ$. However, the polymerization appears to proceed through an isomerization pathway yielding polymer with carbonyls instead of thionocarbonyls. The authors also proposed a reaction mechanism to aid in the explanation of this behavior, Scheme 4.

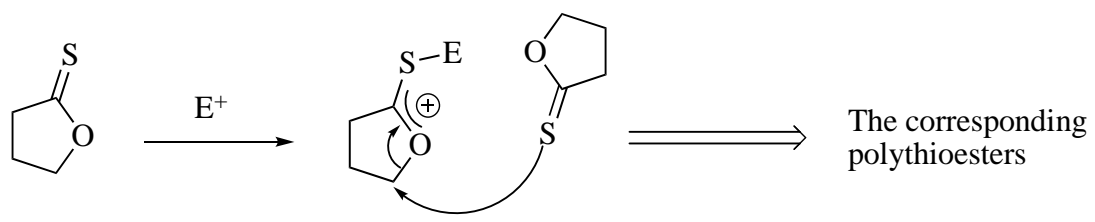

Scheme 4. Proposed mechanism for the cationic ROP of $\mathbf{9 .}$ 
The latest entry into the ROP of thionoesters was published by Datta and Kiesewetter. ${ }^{43}$ Datta synthesized $\varepsilon$-thionocaprolactone (10) following Curphey's method mentioned earlier, except added a distillation via Kugelrohr to obtain extremely pure monomer. Applying the imine bases MTBD, TBD, and DBU to the organocatalyzed ROP of $\mathbf{1 0}$ the authors obtained poly(thionoester)s with quantitative conversion and moderate molecular weights. However, the moderately high molecular weight distribution implies some transesterification of the polymer backbone. Datta found when adding a thiourea (TU) cocatalyst into the system, as in the ROP of $6,{ }^{22}$ the polymerization was much more controlled, and the reaction did not take place with the non-nucleophilic base, BEMP, implying the mechanisms below for the DBU catalyzed and the BEMP/TU cocatalyzed ROP of $\mathbf{1 0}$, Scheme 5. Notably, the

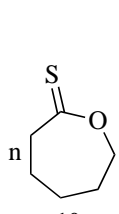

10

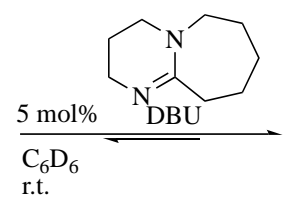

r.t.
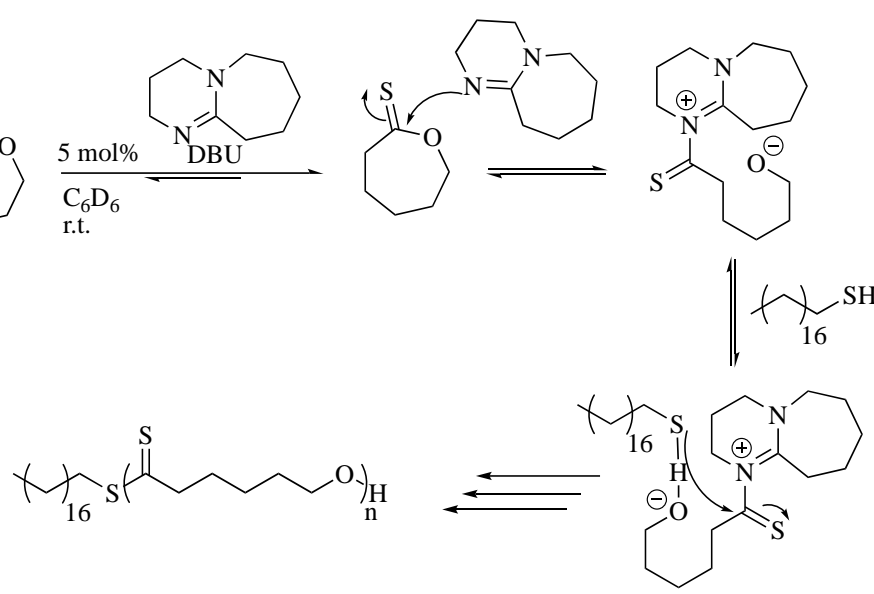

Scheme 5. Proposed mechanism for the DBU catalyzed ROP of $\mathbf{1 0 .}$

authors discovered the ring strain of $\mathbf{1 0}$ is akin to that of $\varepsilon-\mathrm{CL}$ and therefore decided to copolymerize the two monomers to create the first copolymer featuring both carbonyl and thiocarbonyl moieties in the polymer structure, Table 7.

Of all the polymerization techniques available to the modern chemist, organocatalytic mediated living ROP appears to be one of the most robust and controlled synthetic routes to obtaining poly(thioester)s. Strong nucleophilic bases paired with a $\mathrm{H}$-bond donating thiourea co-catalyst provides molecular weight control via $M / I$ feed ratio, narrow to moderate $\boxminus$, allows 
polymerizations to be conducted at ambient temperature (depending on ring size), and is applicable to a wide range of cyclic thio- and thionoesters.

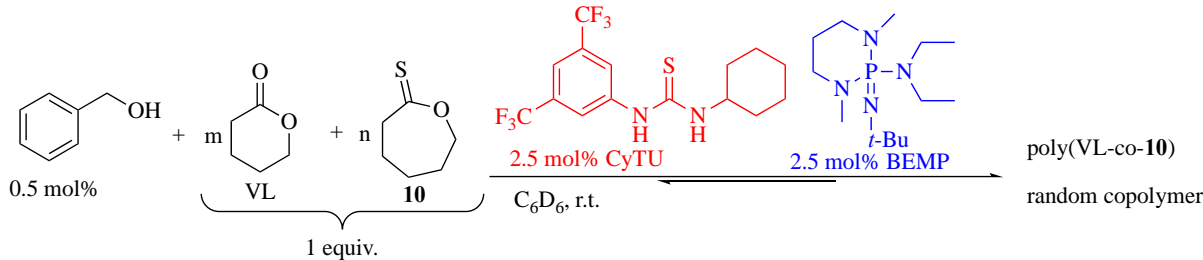

\begin{tabular}{|c|c|c|c|c|c|c|c|c|c|}
\hline entry & $\begin{array}{c}10 \\
(\% \text { feed })\end{array}$ & $\begin{array}{c}\mathrm{VL} \\
(\% \text { feed })\end{array}$ & $\begin{array}{l}\text { time } \\
\text { (h) }\end{array}$ & $\begin{array}{c}\text { conv. }^{b} \\
(\%)\end{array}$ & $M_{\mathrm{n}}{ }^{c}$ & $M_{\mathrm{w}} / M_{\mathrm{n}}{ }^{c}$ & $\begin{array}{l}T_{\mathrm{m}}{ }^{d} \\
\left({ }^{\circ} \mathrm{C}\right)\end{array}$ & $\begin{array}{l}T_{\mathrm{c}}^{d} \\
\left({ }^{\circ} \mathrm{C}\right)\end{array}$ & $\begin{array}{l}T_{\mathrm{deg}^{e}} \\
\left({ }^{\circ} \mathrm{C}\right)\end{array}$ \\
\hline 1 & 0 & 100 & 5 & $0: 93$ & 12,300 & 1.06 & 53 & 27 & 380 \\
\hline 2 & 5 & 95 & 4 & $56: 90$ & 19,600 & 1.02 & 49 & 22 & 440 \\
\hline 3 & 10 & 90 & 5 & 73:93 & 19,200 & 1.02 & 43 & 22 & 360 \\
\hline 4 & 20 & 80 & 4 & $56: 90$ & 19,200 & 1.03 & 40 & 8 & 340 \\
\hline 5 & 30 & 70 & 5 & 79:96 & 18,200 & 1.05 & 31 & -8 & 320 \\
\hline 6 & 50 & 50 & 5 & $95: 92$ & 29,800 & 1.25 & 18 & $\mathrm{n} / \mathrm{a}$ & 310 \\
\hline $7^{f}$ & 100 & 0 & 7 & $89: 0$ & 20,900 & 1.10 & 9 & $\mathrm{n} / \mathrm{a}$ & 260 \\
\hline
\end{tabular}

Table 7. Copolymers of $\mathbf{1 0}$ and VL with varying monomer feeds. ${ }^{a}$ a Polymerization conditions: $4 \mathrm{M}([\mathrm{vl}]=[10])\left(2 \mathrm{mmol}\right.$ total), $2.5 \mathrm{~mol} \%$ CyTU/BEMP (each), $0.5 \mathrm{~mol} \%$ benzyl alcohol in $\mathrm{C}_{6} \mathrm{D}_{6}$. ${ }^{b}$ Percent conversion to polymer obtained by ${ }^{1} \mathrm{H}$ NMR. ${ }^{c}$ Determined by $\mathrm{GPC}\left(\mathrm{CH}_{2} \mathrm{Cl}_{2}\right)$ vs polystyrene standards. ${ }^{d}$ Determined by DSC $\left(\mathrm{N}_{2}\right)$; no $T_{\mathrm{g}}$ were observed $>-70 \stackrel{\circ}{ } \mathrm{C}$, the limit of our DSC. ${ }^{e}$ Determined by TGA $\left(\mathrm{N}_{2}\right)$. ${ }^{f}$ Polymerization conditions: $10(2 \mathrm{M}, 1 \mathrm{mmol}), 5 \mathrm{~mol} \%$ CyTU/BEMP (each), $1 \mathrm{~mol} \%$ benzyl alcohol in $\mathrm{C}_{6} \mathrm{D}_{6} . \mathrm{n} / \mathrm{a}=$ not observed above $-70^{\circ} \mathrm{C}$, the limit of our DSC. 


\section{THIOCARBONATES}

Cyclic carbonates are another group of monomers that provide robust polymers with unique characteristics. Much like cyclic ester monomers, if a sulfur atom is substituted for one or more of the oxygens in the carbonate monomer the properties change drastically; providing significant optical and tunable properties when copolymerized with other monomers. There exist three versions of the cyclic thiocarbonate monomer; tri-, di-, and monothiocarbonates with isomeric variations amongst the aforementioned groups.

Braun and Kissel appear to have reported the first synthesis of a cyclic trithiocarbonate; albeit accidently. ${ }^{2,44}$ Through the polycondensation of an $\alpha, \omega$-dithiol with $\mathrm{CS}_{2}$ and catalyzed via tertiary amines Braun and Kissel synthesized a myriad of poly(alkylene trithiocarbonate)s. ${ }^{2,44}$ While analyzing the polycondensation reactions, the authors discovered the major byproducts to be the 5- and 6-membered cyclic trithiocarbonates. While studying the synthesis of a poly(trithiocarbonate) Leung et al. also synthesized the 5- and 6- membered cyclic trithiocarbonates as a byproduct. ${ }^{45}$ Sometime later the Endo group successfully synthesized various cyclic trithiocarbonates in excellent yields. ${ }^{46}$ Motokucho et al. treated the respective cyclic ether with $\mathrm{CS}_{2}$ catalyzed by a titanium complex. ${ }^{46}$ After varying the feed ratio ( $\mathrm{CS}_{2}$ /oxetane/catalyst) the authors optimized reaction conditions that yielded product in excellent yields ( 90\%). Noteably, Motokucho et al. applied these conditions to various cyclic ethers to obtain a small library of functionalized cyclic trithiocarbonates. Most recently (2014), Soleiman-Beigi and Taherinia synthesized the 5-, 6-, and 7-membered cyclic trithiocarbonates by treating dialkyl halides with $\mathrm{CS}_{2}$ and catalyzing the reaction with imidazole at $45^{\circ} \mathrm{C}$ in atmospheric conditions. Of all the synthetic routes to cyclic trithiocarbonates mentioned above, none probe the ROP of their monomers. It appears only one group has studied the ROP of a trithiocarbonate; ethylenetrithiocarbonate (ETTC). ${ }^{47}$ Soga et al. synthesized ETTC via the Braun and Kissel method. ${ }^{44}$ The authors examined the reaction solvent free and with the same catalysts applied to the copolymerization of $\mathrm{CS}_{2}$ and ethylenesulfide mentioned in their paper. However, they found only sulfuric acid $\left(\mathrm{H}_{2} \mathrm{SO}_{4}\right)$ effected the ROP of ETTC and yielded a brown, 
viscous, low weight material. ${ }^{47}$ Unlike the cyclic trithiocarbonates there appears to be significantly more research done into the ROP of di- and monothiocarbonates.

One lab appears to have conducted all published research on the ROP of dithiocarbonates. The Endo lab has done extensive work with the synthesis and polymerization of mono-, di-, and trithiocarbonates. Kihara et al. successfully synthesized a functionalized 5-membered cyclic dithiocarbonate, 5-(phenoxymethyl)-1,3-oxathiolane-2-thione (11), by treating the respective oxirane with $\mathrm{CS}_{2}$ catalyzed by sodium iodide (Nal) at ambient temperature in THF to afford $\mathbf{1 1}$ in moderate yields. ${ }^{48}$ This reaction also afforded a symmetrical cyclic trithiocarbonate as a byproduct; 5-(phenoxymethyl)-1,3-dithiolane-2-thione (12). In an effort to maximize the yield of 11, the authors applied a myriad of solvents and catalysts to the synthesis. Less polar solvents yield more of 11. However, it was found that protic solvents, like methanol, decrease the selectivity for 11. The authors also discovered Lewis acidic lithium salts ( $\mathrm{LiBr}, \mathrm{LiCl}, \mathrm{Lil})$ showed higher catalytic activity than non-Lewis acidic quaternary ammonium salts; of which afforded no catalytic activity. Notably, the authors also synthesized a small library of functionalized cyclic dithiocarbonates as seen in Table 8. Choi et al. successfully synthesized $\mathbf{1 1}$ and completed the cationic ROP. ${ }^{49-51}$ By reacting glycidyl phenyl ether and $\mathrm{CS}_{2}$ in the presence of $\mathrm{LiBr} \mathbf{1 1}$ was synthesized in excellent yields. ${ }^{49}$ By treating 11 with several cationic catalysts $\left(\mathrm{ZnCl}_{2}, \mathrm{TfOH}\right.$, TfOMe, TfOEt) the authors synthesized isomers of $\mathbf{1 1}$ and poly(dithiocarbonate) with excellent yields, moderate molecular weights, and low to moderate $Đ$, Table 9. When $\mathrm{ZnCl}_{2}$ and $\mathrm{TfOH}$ were applied as cationic initiators the reaction yielded 4-(phenoxymethyl)-1,3-dithiolane-2-one as the major product. However, the ROP of $\mathbf{1 1}$ proceeded when TfOMe and TfOEt were used as initiators, albeit with isomerization. Unfortunately, the thiocarbonyl isomerized to the carbonyl during polymerization and the authors provided a plausible mechanistic explanation, Scheme $6 .^{49}$ 


entry
1

Table 8. Reaction of various oxiranes and carbon disulfide. ${ }^{a}$ The isomers were not separable. ${ }^{b} \mathrm{~A} 4: \mathrm{B} 4: \mathrm{C} 4$ estimated by ${ }^{1} \mathrm{H}$ NMR spectra.<smiles>S=C1OC(COc2ccccc2)CS1</smiles>

\begin{tabular}{|c|c|c|c|c|c|c|c|c|}
\hline entry & catalyst & solvent & $\begin{array}{l}\text { temp. } \\
\left({ }^{\circ} \mathrm{C}\right)\end{array}$ & $\begin{array}{l}\text { time } \\
\text { (h) }\end{array}$ & $\begin{array}{l}\text { conv. } \\
\text { (\%) }\end{array}$ & $\begin{array}{l}\text { yield } \\
(\%)\end{array}$ & $\begin{array}{c}\text { product } \\
\text { ratio }{ }^{b} \mathrm{~B} A \\
(\%)\end{array}$ & $\begin{array}{c}M_{\mathrm{n}}\left(M_{\mathrm{w}} / M_{\mathrm{n}}\right)^{c} \text { of } \\
\mathrm{A}\end{array}$ \\
\hline 1 & $\mathrm{ZnCl}_{2}$ & $\mathrm{PhCl}$ & 80 & 2 & 43 & $--d$ & $0: 100$ & -- \\
\hline 2 & $\mathrm{ZnCl}_{2}$ & $\mathrm{PhCl}$ & 80 & 12 & 100 & $87^{e}$ & $0: 100$ & -- \\
\hline
\end{tabular}




$\begin{array}{ccccccccc}3 & \text { TfOH } & \mathrm{CDCl}_{3} & 60 & 1 & 55 & --^{d} & 1: 99 & -- \\ 4 & \text { TfOH } & \mathrm{CDCl}_{3} & 60 & 2 & 100 & 86^{e} & 2: 98 & -- \\ 5 & \text { TfOMe } & \text { bulk } & 60 & 3 & 100 & 92^{f} & 96: 4 & 11,000(1.25) \\ 6 & \text { TfOEt } & \text { bulk } & 60 & 2 & 73 & 68^{f} & 98: 2 & 9,000(1.23) \\ 7 & \text { TfOEt } & \text { bulk } & 60 & 3 & 100 & 98^{f} & 98: 2 & 10,500(1.25) \\ 8 & \text { TfOEt } & \mathrm{PhCl} & 60 & 1 & 46 & 48^{f} & 97: 3 & 6,700(1.25) \\ 9 & \text { TfOEt } & \mathrm{PhCl} & 60 & 2 & 100 & 94^{f} & 94: 6 & 8,700(1.46) \\ 10 & \text { TfOEt } & \mathrm{PhCl} & 60 & 19 & 100 & --{ }^{d} & 70: 30 & 8,000(1.81) \\ 11 & \text { TfOEt } & \mathrm{PhCl} & 60 & 67 & 100 & --^{d} & 56: 44 & 5,900(1.57)\end{array}$

Table 9. Cationic isomerization and polymerization of $11 .^{a}$ a Catalyst; $2 \mathrm{~mol} \%$ vs. 11. Concentration of 11 in solution polymerization; 3M (runs 1-4 and 8-11). ${ }^{b}$ Estimated by ${ }^{1} \mathrm{H}$ NMR. ${ }^{c}$ Estimated by GPC (elutent, THF, based on polystyrene standards). ${ }^{d}$ Not determined. ${ }^{e}$ Isolated by preparative HPLC. ${ }^{f} n$-Hexane-insoluble part.

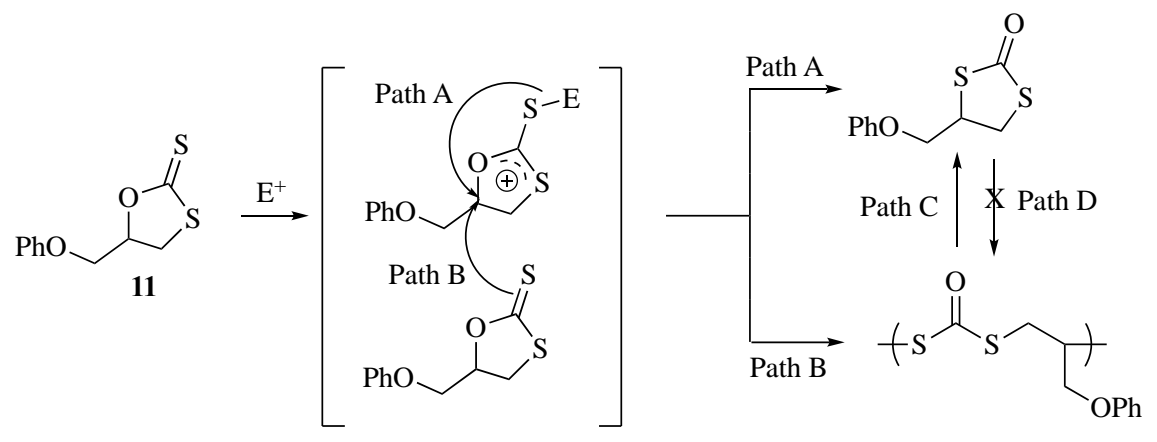

Scheme 6. Proposed mechanism for the cationic isomerization and polymerization of 11.

Choi and coworkers continued their work into the cationic ROP of cyclic dithiocarbonates by expanding their monomer library ${ }^{50,51}$ and determining the dependence ${ }^{50}$ of the ring-opening polymerization of dithiocarbonate monomers on cationic catalysts. Mechanistic studies lead the authors to propose a mechanism for the cationic ROP of several dithiocabonates seen in Scheme $7 .{ }^{50}$ To follow up the aforementioned work the Endo group studied the 

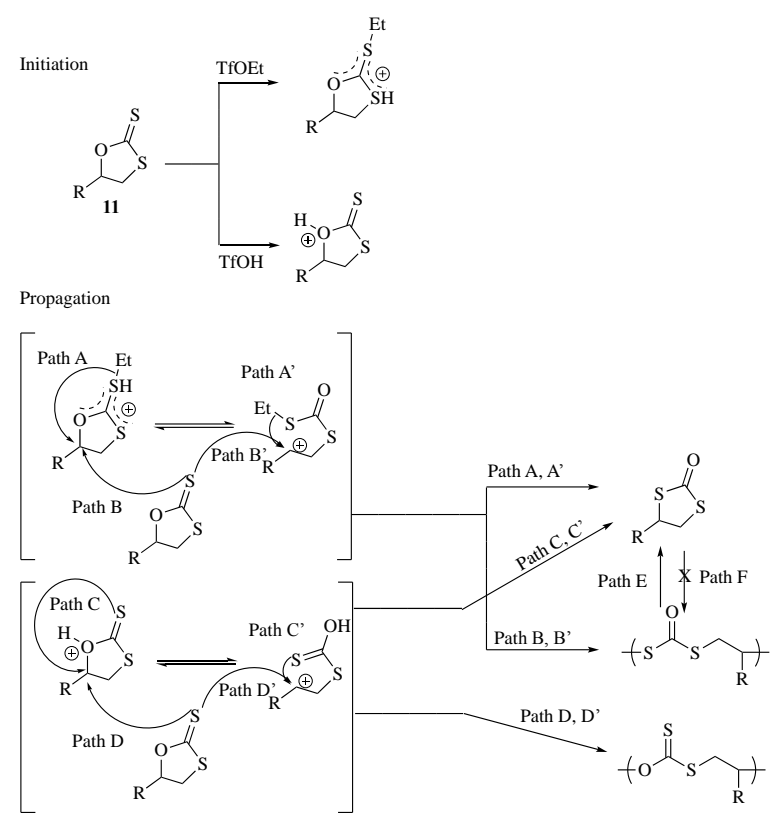

Scheme 7. Proposed mechanism for the cationic isomerization and polymerization of $\mathbf{1 1}$ using TfOEt or TfOH as catalysts supported by mechanistic studies.

depolymerization of certain dithiocarbonates. ${ }^{52,53}$ The authors applied $\mathrm{TfOH}, \mathrm{TfOMe}, \mathrm{Et}_{3} \mathrm{~N}$, and tert-BuOK as catalysts in chlorobenzene $(\mathrm{PhCl})$ and tetrahydrofuran (THF) at various temperatures (20-60ㄷ) and reaction times (24-96 hours); Table 10. Cationic depolymerization

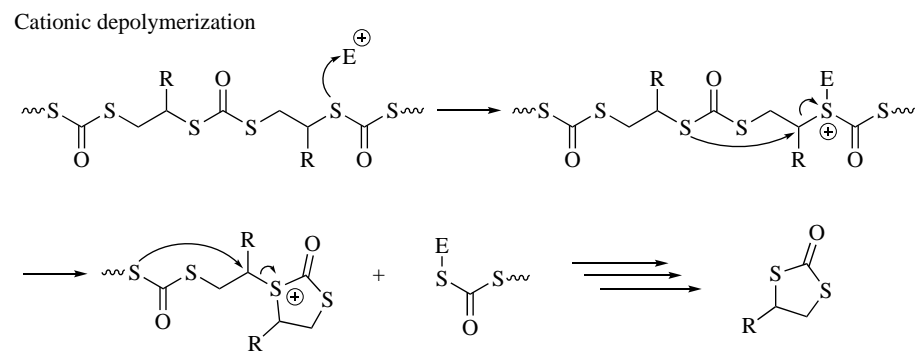

Anionic depolymerization

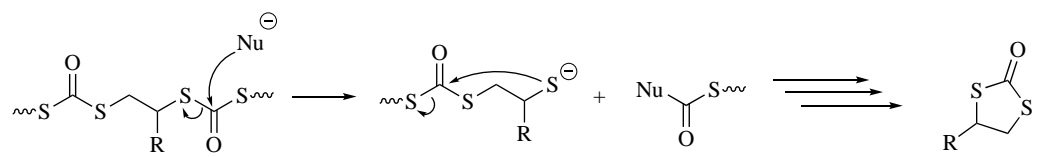

$\begin{array}{lcccccc}\text { entry } & \begin{array}{c}\text { initiator }^{b} \\ (\mathrm{~mol} \mathrm{\% )}\end{array} & \text { solvent }^{c} & \text { temp. }\left({ }^{\circ} \mathrm{C}\right) & \text { time }(\mathrm{h}) & \begin{array}{r}\text { isomer } \\ \text { yield }^{d}\end{array} & M_{\mathrm{n}}\left(M_{\mathrm{w}} / M_{\mathrm{n}}\right)^{e} \\ 1 & \text { TfOMe (5) } & \mathrm{PhCl} & 60 & 96 & 38 & 8,000(1.7) \\ 2 & \text { TfOMe (20) } & \mathrm{PhCl} & 60 & 96 & 83 & 3,000(1.4)\end{array}$




$\begin{array}{ccccccc}3 & \mathrm{TfOH}(5) & \mathrm{PhCl} & 60 & 96 & 34 & 5,000(1.9) \\ 4 & \mathrm{TfOH}(20) & \mathrm{PhCl} & 60 & 96 & 35 & 2,000(1.6) \\ 5 & \mathrm{Et}_{3} \mathrm{~N}(5) & \mathrm{PhCl} & 20 & 24 & 85 & 4,000(1.3) \\ 6 & \mathrm{Et}{ }_{3} \mathrm{~N}(5) & \mathrm{THF} & 20 & 24 & 92 & 4,000(1.5) \\ 7 & t \text {-BuOK (5) } & \mathrm{PhCl} & 20 & 24 & 100 & -- \\ 8 & t \text {-BuOK (5) } & \mathrm{THF} & 20 & 24 & 100 & --\end{array}$

Table 10. The depolymerization of a polythiodicarbonate $\left(M_{n}=11,000, M_{w} / M_{n}=1.2\right) \cdot{ }^{a} a^{a}$ Polymer synthesized by cationic polymerization of $A$ with 2 mol\% TfOMe at $60{ }^{\circ} \mathrm{C}$ for $3 \mathrm{~h}$ in $93 \%$ yield. ${ }^{b}$ Calculated from $\mathrm{mol} \%$ vs repeating unit of polydithiocarbonate. ${ }^{c}$ Concentration of the repeating unit of polydithiocarbonate: $3 \mathrm{M} .{ }^{d}$ Determined by ${ }^{1} \mathrm{H}$ NMR. ${ }^{e}$ Determined by GPC, polystyrene calibration, eluted with THF.

conditions with high loadings of TfOMe (20 mol\%) yielded the respective monomer(s) whereas lower loadings produced oligomers and monomer. When studying the anionic depolymerization the authors found $5 \mathrm{~mol} \% \mathrm{Et}_{3} \mathrm{~N}$ and $t$-BuOK yielded 1,3-dithiolane-2-one derivitives; the latter being quantitative, Table 10, entries 6-8.

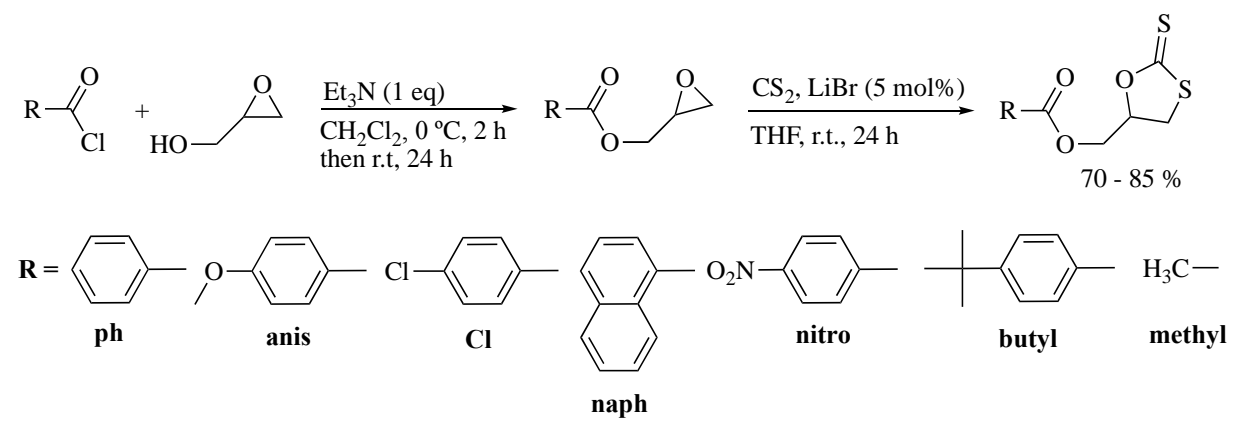

Scheme 8. Reaction conditions for the formation of a functionalized dithiocarbonate.

Through the work of Steblyanko et al., the Endo lab furthered their work in the cationic ROP of 5-membered dithiocarbonates by reacting $\mathrm{CS}_{2}$ with benzoic, $p$-anisic, $p$-chlorobenzoic, 1 naphthalenecarboxylic, $p$-nitrobenzoic, and $p$-(tert-butyl)benzoic glycidyl esters in the presence of $\mathrm{LiBr}$ (Scheme 8); further expanding the monomer library. ${ }^{54}$ The polymers were synthesized in the presence of $\mathrm{TfOH}$ and $\mathrm{TfOMe}$ at $80^{\circ} \mathrm{C}$ in good yields, moderate molecular weights, and fairly narrow $Đ$, Table 11 . Throughout the course of this study, the authors, through 
experimentation and theoretical calculations, reported a dependence on the substituent in the reactivity of the monomers and found the rate of polymerization decreased in the order of $p$ chlorobenzoic $\geq$ benzoic $>1$-naphthalenecarboxylic $>p$-nitro-benzoic $>p$-tert-butylbenzoic $>p$ anisic. $^{54}$

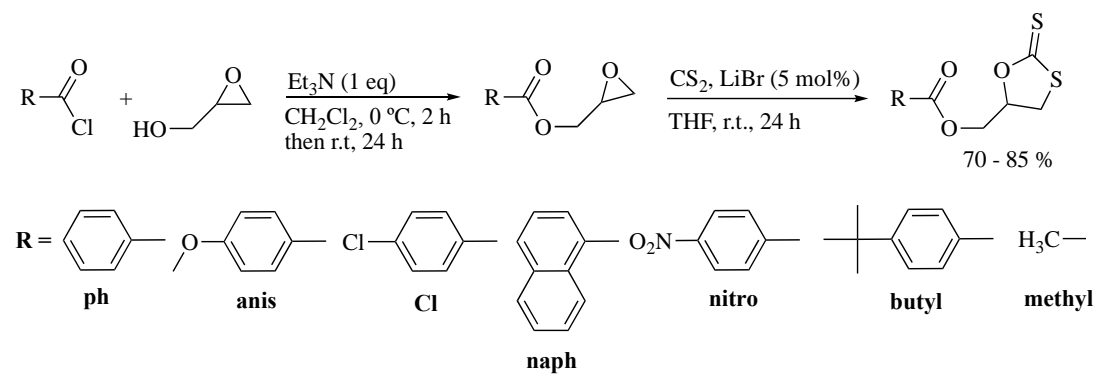

\begin{tabular}{|c|c|c|c|c|c|c|c|c|}
\hline entry & $\begin{array}{c}\text { monomer } \\
(\mathrm{R})\end{array}$ & $\begin{array}{c}\text { initiator (mol } \\
\% \text { ) }\end{array}$ & $\begin{array}{c}T \\
\left({ }^{\circ} \mathrm{C}\right)\end{array}$ & $\begin{array}{l}\text { time } \\
\text { (h) }\end{array}$ & $\begin{array}{l}\text { conv. } \\
\text { (\%) }\end{array}$ & $\begin{array}{l}\text { yield } \\
(\%)\end{array}$ & $M_{\mathrm{n}}{ }^{d}$ & $M_{\mathrm{w}} / M_{\mathrm{n}}{ }^{d}$ \\
\hline 1 & ph & TfOH (2.0) & rt & 4 & 60 & 58 & 24,900 & 1.29 \\
\hline 2 & ph & TfOMe (3.3) & rt & 35 & 100 & $<99$ & 7,700 & 1.19 \\
\hline 3 & ph & TfOH (2.0) & 60 & 1 & 100 & 98 & 16,700 & 1.27 \\
\hline 4 & anis & TfOMe (2.6) & 60 & 11 & 100 & 100 & 9,200 & 1.24 \\
\hline 5 & anis & TfOMe (1.8) & rt & 72 & 65 & 62 & 8,900 & 1.08 \\
\hline 6 & anis & TfOMe (8.0) & rt & 28 & 98 & $<98$ & 3,600 & 1.11 \\
\hline 7 & anis & TfOMe (2.6) & 45 & 12 & 94 & 92 & 9,600 & 1.08 \\
\hline 8 & anis & TfOMe (2.6) & 60 & 3 & 90 & 86 & 11,000 & 1.08 \\
\hline 9 & anis & TfOMe (3.3) & 60 & 10 & 100 & $<100$ & 8,400 & 1.20 \\
\hline 10 & anis & TfOMe (4.0) & 80 & 3 & 100 & $<99$ & 6,900 & 1.17 \\
\hline 11 & $\mathrm{Cl}$ & TfOMe (2.5) & rt & 120 & 97 & 96 & 14,800 & 1.25 \\
\hline 12 & $\mathrm{Cl}$ & TfOMe (2.5) & 45 & 6 & 100 & 100 & 12,900 & 1.17 \\
\hline 13 & $\mathrm{Cl}$ & TfOMe (3.3) & 60 & 2 & 100 & 100 & 8,500 & 1.22 \\
\hline 14 & naph & TfOMe (8.8) & 60 & 2 & 100 & 100 & 3,400 & 1.08 \\
\hline 15 & nitro & TfOMe (4.0) & 60 & 4 & 100 & 88 & 1,200 & 1.40 \\
\hline 16 & butyl & TfOMe (4.0) & 60 & 2 & 53 & $<50$ & 3,600 & 1.20 \\
\hline
\end{tabular}




$\begin{array}{rrrrrrrrr}17 & \text { methyl } & \text { TfOH (2.0) } & 60 & 6 & 100 & 59 & 4,600 & 1.60 \\ 18 & \text { methyl } & \text { TfOMe (2.0) } & 60 & 12 & 100 & 61 & 4,800 & 1.61\end{array}$

Table 11. Cationic ROPa of 5-membered cyclic ditjiocarbonates. a $1 \mathrm{M}$ solution in $\mathrm{PhCl}$ for ph and methyl; $0.5 \mathrm{M}$ for anis; and $0.25 \mathrm{M}$ for $\mathrm{Cl}$, naph, nitro, and butyl. ${ }^{b}$ Estimated by ${ }^{1} \mathrm{H}$ NMR. ${ }^{c}$ $n$-Hexane insoluble part. ${ }^{d}$ Estimated by GPC based on polystyrene standards with THF as eluent. ${ }^{e}$ Temperature with $5 \%$ weight loss estimated by TGA under $\mathrm{N}_{2}$; ND = not determined.

Although the ROP work for tri- and dithiocarbonates is somewhat limited, monothiocarbonates appear to have been extensively researched. Soga et al. reported the first ROP of a monothiocarbonate, ethylene monothiocarbonate (EMTC). ${ }^{55}$ Treating diethyl carbonate with 2mercapto ethanol in the presence of thorium nitrate the cyclic ethylene monothiocarbonate was produced in good yields. ${ }^{55}$ Polymerizations were carried out in a stainless steel reactor with several different catalysts at $80^{\circ} \mathrm{C}$ to yield a mostly white solid, Table 12 . The authors did not conduct mechanistic studies or provide any detailed results; leaving it for another paper that does not appear to have materialized. Kricheldorf and Damrau synthesized and applied the cationic ROP conditions to 1,3-dioxolane-2-thione, 12.56 Their cationic ROP conditions were all conducted in chloroform at ambient temperatures. Utilizing several different catalysts (TfOMe, $\mathrm{BF}_{3} \mathrm{OEt}_{2}, \mathrm{SnCl}_{4}, \mathrm{BuSnCl}_{3}, \mathrm{Bu}_{2} \mathrm{SnCl}_{2}$ ), the authors reported what looks like erratic data, Table 13. However, upon further study it was found that the "thermal history", or how the monomer was stored prior to use, played a large part in affecting the molecular weights of the poly(thiocarbonate)s produced. Unfortunately, the authors also noted that only poly(mercaptopropanol carbonate)s were formed. 


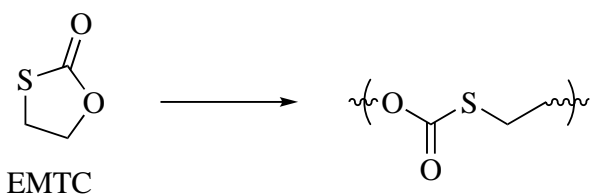

\begin{tabular}{|c|c|c|c|c|c|}
\hline run & catalyst $^{b}$ & $T\left({ }^{\circ} \mathrm{C}\right)$ & yield (mg) & $\mathrm{Mp}\left({ }^{\circ} \mathrm{C}\right)$ & $\frac{\eta_{s p}}{c}(\mathrm{dl} / \mathrm{g})^{c}$ \\
\hline 1 & none & 80 & 0 & -- & -- \\
\hline 2 & $\mathrm{Al}\left(\mathrm{C}_{2} \mathrm{H}_{5}\right)_{3}$ & 80 & 0 & -- & -- \\
\hline 3 & $\mathrm{Al}\left(\mathrm{OC}_{4} \mathrm{H}_{9}\right)_{3}$ & 80 & 100 & 160 & 0.13 \\
\hline 4 & $\mathrm{AlCl}_{3}$ & 80 & 0 & -- & -- \\
\hline 5 & $\mathrm{Zn}\left(\mathrm{C}_{2} \mathrm{H}_{5}\right)_{2}$ & 80 & 25 & 175 & -- \\
\hline 6 & $\mathrm{Cd}\left(\mathrm{C}_{2} \mathrm{H}_{5}\right)_{2}$ & 80 & 30 & 130 & -- \\
\hline 7 & $\mathrm{Ti}\left(\mathrm{OC}_{4} \mathrm{H}_{9}\right)_{3}$ & 40 & 35 & 150 & -- \\
\hline 8 & $\mathrm{Ti}\left(\mathrm{OC}_{4} \mathrm{H}_{9}\right)_{3}$ & 80 & 255 & 90 & -- \\
\hline 9 & $\mathrm{Ti}\left(\mathrm{OC}_{4} \mathrm{H}_{9}\right)_{3}$ & 110 & 410 & 135 & 0.074 \\
\hline 10 & $\begin{array}{c}\mathrm{Mg}\left(\mathrm{OCH}_{3}\right) \\
2\end{array}$ & 80 & 15 & 195 & -- \\
\hline 11 & $\mathrm{NaOCH}_{3}$ & 80 & 40 & 150 & -- \\
\hline 12 & $\mathrm{~N}\left(\mathrm{C}_{2} \mathrm{H}_{5}\right)_{3}$ & 80 & 510 & 205 & 0.068 \\
\hline 13 & $\mathrm{P}\left(\mathrm{C}_{6} \mathrm{H}_{5}\right)_{3}$ & 80 & 430 & 205 & -- \\
\hline 14 & $\mathrm{HCl}$ & 80 & 0 & -- & -- \\
\hline
\end{tabular}

Table 12. Analytical data of polymers made from EMTC. ${ }^{a}$ a Polymerizations carried out for 70 h with $0.97 \mathrm{~g}(9.3 \mathrm{mmol})$ of EMTC. ${ }^{b} 0.1 \mathrm{mmol} .{ }^{c} 6 \mathrm{mg} / \mathrm{mL}$ in $p$-chlorophenol at $160 \stackrel{\circ}{\circ}$. 


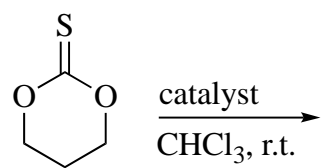

13<smiles>CSC(=O)OCC[Al]</smiles>

Poly1

\begin{tabular}{llccc} 
entry & catalyst & mol ratio (mon/cat) & T (h) & yield (\%) \\
$1^{a}$ & TfOMe & $100 / 1$ & 4 & 49 \\
$2^{a}$ & TfOMe & $100 / 1$ & 8 & 60 \\
$3^{a}$ & TfOMe & $200 / 1$ & 8 & 68 \\
$4^{b}$ & TfOMe & $100 / 1$ & 4 & 95 \\
$5^{b}$ & TfOMe & $100 / 1$ & 8 & 95 \\
$6^{a}$ & $\mathrm{BF}_{3} \cdot \mathrm{OEt}_{2}$ & $100 / 1$ & 4 & 83 \\
$7^{a}$ & $\mathrm{BF}_{3} \cdot \mathrm{OEt}_{2}$ & $100 / 1$ & 8 & 80 \\
$8^{a}$ & $\mathrm{BF}_{3} \cdot \mathrm{OEt}_{2}$ & $200 / 1$ & 8 & 68 \\
$9^{b}$ & $\mathrm{BF}_{3} \cdot \mathrm{OEt}_{2}$ & $100 / 1$ & 4 & 88 \\
10 & $\mathrm{BF}_{3} \cdot \mathrm{OEt}_{2}$ & $100 / 1$ & 8 & 82 \\
$11^{a}$ & $\mathrm{SnCl}_{4}$ & $100 / 1$ & 4 & 50 \\
$12^{a}$ & $\mathrm{SnCl}_{4}$ & $100 / 1$ & 8 & 63 \\
$13^{a}$ & $\mathrm{BuSnCl}_{3}$ & $100 / 1$ & 4 & 47 \\
$14^{a}$ & $\mathrm{BuSnCl}_{3}$ & $100 / 1$ & 64 \\
$15^{a}$ & $\mathrm{Bu}_{2} \mathrm{SnCl}_{2}$ & $100 / 1$ & 60 \\
$16^{a}$ & $\mathrm{Bu}_{2} \mathrm{SnCl}_{2}$ & $100 / 1$ & 87 \\
\hline
\end{tabular}

Table 13. Polymerizations of $\mathbf{1 3}$ initiated by acidic catalysts. ${ }^{a}$ Freshly recrystallized monomer (1 day before polymerization). ${ }^{b}$ Monomer stored in refrigerator prior to polymerization (2 months).

The Endo lab has conducted significant research on the ROP of monothiocarbonates to include 5,5-dimethyl-1,3-dioxan-2-thione (14), ${ }^{57-60}$ 4-benzoyloxymethyl-1,3-dioxolane-2-thione (15), ${ }^{60,61}$ 5-benzoyloxymethyl-5-methyl-1,3-dioxane-2-thione (16), ${ }^{60}$ 4-phenoxymethyl-1,3-dioxolane-2thione $\quad(\mathbf{1 7}),{ }^{61} \quad$ 4,6-dioxatetracyclo-[6.3.1.1. $\left.{ }^{3,10} 0^{3,7}\right]$ tridecane-5-thione $\quad(\mathbf{1 8}),{ }^{62}$ 
tricyclo[3.3.1.1 $\left.1^{3,7}\right]$ decane-2-spiro-4-(1,3-dioxolane-2-thione) $\quad$ (19), ${ }^{63} \quad$ 5,5-(bicyclo[2.2.1]hept-2en-5,5-ylidene)-1,3-dioxane-2-thione $\quad(\mathbf{2 0}),{ }^{64} \quad 5,5-(\quad$ bicycle[2.2.1]heptan-5,5-ylidene)-1,3dioxane-2-thione (21), ${ }^{64}$ and 1,3-dioxepan-2-thione (22). ${ }^{65,66}$ All monomers were synthesized by reacting the respective diol with $\mathrm{CS}_{2}$ in the presence of either $\mathrm{Et}_{3} \mathrm{~N}$ or antipyrine. The polymerization of $\mathbf{1 4}$ was catalyzed by $\mathrm{TfOH}$, TfOMe, $\mathrm{Et}_{3} \mathrm{OBF}_{4}$, or $\mathrm{BF}_{3} \mathrm{OEt}_{2}$ at elevated temperatures $\left(30-80^{\circ} \mathrm{C}\right)$ under a nitrogen atmosphere. ${ }^{57-60}$ In all cases the polymerizations exhibited narrow molecular weight distribution (Table 14) and good solubility in typical organic<smiles>CC(C)(C)COC(=O)SNC1CCCC1</smiles>

$\begin{array}{lccccccc}\text { entry } & \text { solvent } & \text { initiator } & \mathrm{T}(\mathrm{h}) & T\left({ }^{\circ} \mathrm{C}\right) & \text { conv. }^{b}(\%) & M_{\mathrm{n}}{ }^{c} & M_{\mathrm{w}} / M_{\mathrm{n}}{ }^{c} \\ 1 & \mathrm{CH}_{2} \mathrm{Cl}_{2} & \mathrm{Et}_{3} \mathrm{OBF}_{4} & 24 & 30 & >99 & 13,200 & 1.04 \\ 2 & \mathrm{CHCl}_{3} & \mathrm{Et}_{3} \mathrm{OBF}_{4} & 20 & 40 & >99 & 16,800 & 1.04 \\ 3 & \mathrm{PhCl} & \mathrm{TFOMe} & 12 & 80 & >99 & 11,200 & 1.15 \\ 4 & \mathrm{PhCl} & \mathrm{TfOH} & 12 & 80 & >99 & 13,900 & 1.13 \\ 5 & \mathrm{PhCl} & \mathrm{BF}_{3} \mathrm{OEt} & 12 & 80 & >99 & 31,000 & 1.08\end{array}$

Table 14. Cationic ROP of $14 .^{a}{ }^{a}$ Monomer, $0.25 \mathrm{mmol}$; solvent, $0.25 \mathrm{~mL}$; catalyst (initiator), 0.005 mmol. ${ }^{b}$ Determined by ${ }^{1} \mathrm{H}$ NMR spectroscopy measured in $\mathrm{CDCl}_{3} .{ }^{c}$ Estimated from GPC eluted with THF based on polystyrene standards.

solvents. However, the authors noted erratic $M_{n}$ 's amongst the catalysts presumably due to the difference in initiation efficiencies. While interpreting spectra, the authors noted a scrambling of the thionocarbonyl group to a carbonyl group and proposed a mechanism to explain their results, Scheme 9. The authors also studied the photo-initiated

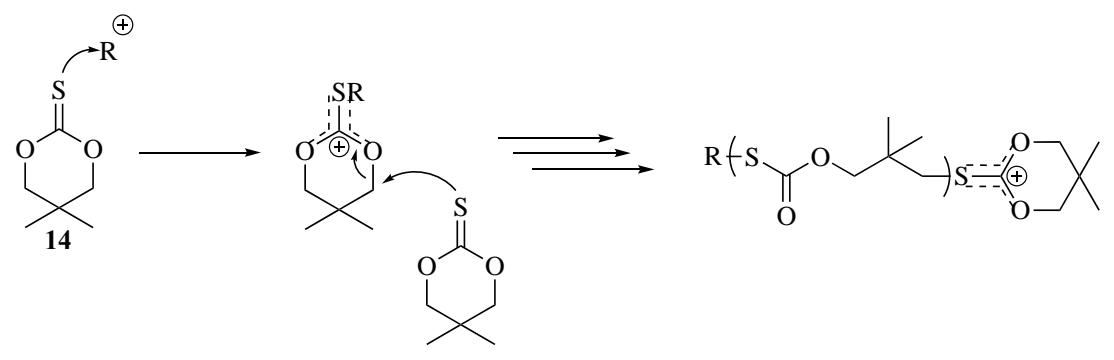


Scheme 9. Proposed isomerization mechanism for the cationic ROP of 14.

ROP of $\mathbf{1 4}$ in the presence of onium salts initiated by 2.5 hours of irradiation at $\lambda=300 \mathrm{~nm}^{59}$ The authors reported moderate $M_{n}$ 's, moderate conversions, and broad molecular weight distributions. Notably, the reactions were conducted at ambient temperature in the presence of air.

To further probe the cationic ROP of monothiocarbonates the Endo group studied how neighboring groups effected the ROP of 5- (15 and 17) and 6-membered monothiocarbonates (16). ${ }^{60,61}$ By comparing the reactivity of neighboring ester groups to neighboring phenoxy groups attached to $\mathbf{1 7}$ the authors, again, observed isomerization of the thiocarbonyl to a carbonyl in the polymer. The reactivity of the $\mathbf{1 7}$ almost equaled that of $\mathbf{1 5}$. However, the phenoxy substituted poly(thiocarbonate)s had a broader distribution of molecular weights than the ester substituted poly(thiocarbonate)s. When comparing the neighboring group participation of the cationic ROP of the 6-membered thiocarbonates $(\mathbf{1 4}, \mathbf{1 5}, \mathbf{1 6})$ the authors discovered the fastest rates occurred when the ester group was alpha to the oxygen. ${ }^{60}$ Although, the cationic ROP of all monomers produced poly(thiocarbonate)s that exhibited living characteristics; i.e., narrow molecular weight distribution and controlled molecular weights. However, the polymerization was accompanied by isomerization of the thiocarbonate group. ${ }^{60}$

The Endo group also studied the anionic ROP of cyclic thiocarbonates containing spiro linked norborane and norborene functional groups. ${ }^{64}$ Kakimoto et al. noted when DBU was applied to the anionic ROP of 5,5-(bicyclo[2.2.1]hept-2-en-5,5-yildene)-1,3-dioxane-2-thione (20) and 5,5(bicyclo[2.2.1]heptan-5,5-yildene)-1,3-dioxane-2-thione (21) afforded polymer in all cases except run 4 (Table 15) with mildly broad molecular weight distributions, low yields, and

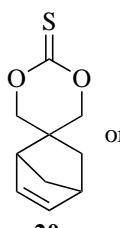

20

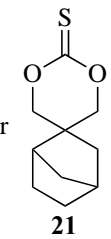

$\mathrm{T}(\mathrm{h})$ $\underset{\text { bulk or solvent }}{\text { DBU }(4 \mathrm{~mol} \%)}$ $120^{\circ} \mathrm{C}$

entry monomer solvent

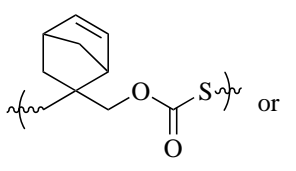

P20

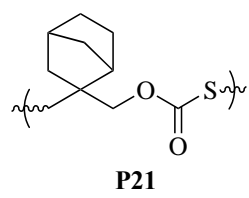

P21 


$\begin{array}{rrrcrrr}1 & \mathbf{2 0} & 1 & -- & 25 & 15,200 & 1.48 \\ 2 & \mathbf{2 0} & 12 & \text { toluene } & 38 & 8,800 & 1.44 \\ 3 & \mathbf{2 0} & 12 & \text { DMF } & 38 & 8,600 & 1.47 \\ 4 & \mathbf{2 1} & 1 & -- & & \text { no reaction } & \\ 5 & \mathbf{2 1} & 12 & \text { toluene } & 8 & 7,100 & 1.43 \\ 6 & \mathbf{2 1} & 12 & \text { DMF } & 22 & 11,800 & 1.40\end{array}$

Table 15. Anionic ROP of 20 and $21 .^{a}$ a Temperature, $120^{\circ} \mathrm{C}$; monomer, $1.00 \mathrm{mmol}$; solvent, $0.20 \mathrm{~mL}$; DBU, 0.040 mmol. ${ }^{b}$ Calculated from products isolated with HPLC eluted with $\mathrm{CHCl}_{3}$. ${ }^{c}$ Estimated from GPC eluted with THF at $40{ }^{\circ} \mathrm{C}$ based on polystyrene standards.

isomerization of the thiocarbonyl to its respective carbonyl. Notably, the authors observed volume expansion of the polymer when the densities were determined. When compared to the density of the respective poly(carbonate)s the poly(thiocarbonate)s yielded polymer with a volume change greater than that of poly(carbonate), Table 16.

The same group compared the cationic ROP of a five-membered thionocarbonate containing adamantane moieties. ${ }^{62,63} \quad 4,6$-dioxatetracyclo[6.3.1.1.1.10 $\left.0^{3,7}\right]$ tridecane-5-thione ${ }^{62}$ (18) was initiated by $\mathrm{BF}_{3} \mathrm{OBF}_{4}$, TfOMe, $\mathrm{TfOH}$, or $\mathrm{H}_{2} \mathrm{O} / \mathrm{BF}_{3} \mathrm{OEt}$ to afford poly(thiocarbonate)s in moderate yields, Table 17. Kameshima et al. noted the propagation rate to be slow; possibly due to

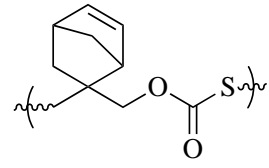

P20

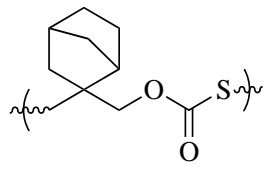

P21

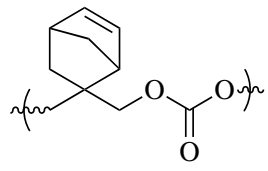

poly(spiro)carbonate

$\begin{array}{lcccc}\text { entry } & \text { polymer } & \text { Volume change }^{a}(\%) & T_{\mathrm{g}}{ }^{b}\left({ }^{\circ} \mathrm{C}\right) & \left.T_{\mathrm{d} 10^{c}}{ }^{\circ} \mathrm{O}\right) \\ 1 & \mathbf{P 2 0} & +12.3 & 82 & 258 \\ 2 & \mathbf{P 2 1} & +12.6 & 82 & 261 \\ 3 & \text { poly(spiro)carbonate } & +8.2 & 108 & 207\end{array}$


Table 16. Properties of P20, P21, and poly(spiro)carbonate. ${ }^{a}$ Calculated by the densities of the monomer and polymer. ${ }^{b}$ Determined by DSC. ${ }^{c}$ Determined by TGA.

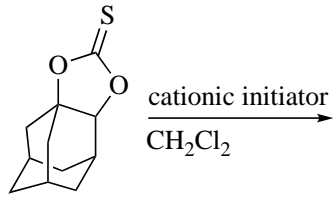

18<smiles>CNC(=O)OC1C2CC3CC(C2)CC1C3</smiles>

\begin{tabular}{lccccc} 
entry & initiator & $T\left(^{\circ} \mathrm{C}\right)$ & conv. $^{b}(\%)$ & $M_{\mathrm{n}}{ }^{\mathrm{C}}$ & $M_{\mathrm{w}} / \mathrm{Mn}^{c}$ \\
1 & $\mathrm{TfOH}$ & 0 & 45 & 1,600 & 1.60 \\
2 & $\mathrm{TfOH}$ & 30 & $>99$ & 3,600 & 1.49 \\
3 & $\mathrm{TfOMe}$ & 0 & 44 & 2,100 & 1.46 \\
4 & $\mathrm{TfOMe}$ & 30 & 95 & 3,600 & 1.48 \\
5 & $\mathrm{Et}_{3} \mathrm{OBF}$ & & \\
6 & $\mathrm{Et}_{3} \mathrm{OBF}$ & & 28 & 1,100 & 1.58 \\
7 & 30 & 45 & 2,800 & 1.81 \\
8 & $\mathrm{BF}_{3} \mathrm{OEt}$ & & & trace & \\
\hline $\mathrm{BF}_{3} \mathrm{OEt}_{2}$ & 30 & 74 & 10,600 & 1.44
\end{tabular}

Table 17. Cationic ROP of 18. ${ }^{a}$ a Solvent, $\mathrm{CH}_{2} \mathrm{Cl}_{2}$; monomer, $1 \mathrm{M}$; reaction time, $24 \mathrm{~h}$; initiator, 2 mol\%. ${ }^{b}$ Determined by ${ }^{1} \mathrm{H}$ NMR; comparison of the residual monomer at $4.67 \mathrm{ppm}$ with the adamantyl protons of the monomer and polymer found around $1.2-2.8 \mathrm{ppm}$. The authors note that they considered the polymer yield equal to the monomer conversion, because no low molecular weight compound other than monomer was detected by SEC and NMR. ${ }^{c}$ Determined from GPC eluted with THF based on polystyrene standards.

competing backbiting or transesterification reactions taking place. Utilizing NMR, IR, and $a b$ initio computational methods, the authors showed the polymerization to be selective in its scission of the ring-opening direction, Scheme 10. Notably, the polymerization proceeded with

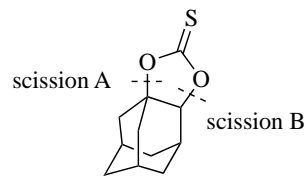

18

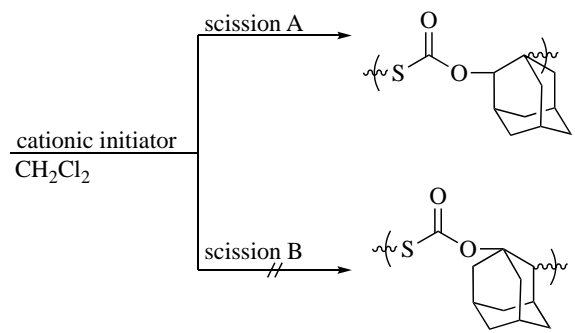

Scheme 10. Proposed mechanism for the cationic ROP of 18. 
up to a $14 \%$ volume expansion. The spiro-linked tricycle[3.3.1.1 $\left.1^{3,7}\right]$ decane-2-spiro-4'-(1.3dioxolane-2'-thione) $)^{63}$ (19) was subjected to the same initiators as 17 along with $p$ - $\mathrm{TsOH}, \mathrm{TiCl}_{4}$, and $\mathrm{CH}_{3}$ l. However, 19 did not undergo ROP with cationic initiators; most likely due to the steric bulk of the spiro-linked admantane moiety. The authors did notice when $\mathbf{1 9}$ was added to the polymerization of certain 5- and 6-membered thiocarbonates (1,3-dioxolane-2-thione, 5-methyl1,3-dioxane-2-thione, 13, 14) a copolymer was synthesized. However, the copolymer resulted in low molecular weight co-poly(thiocarbonate)s with broad molecular weight distributions (Table 18) and other unidentified products. Molecular orbital calculations utilizing the ab initio method were conducted to identify the ring-opening direction, Scheme 11.

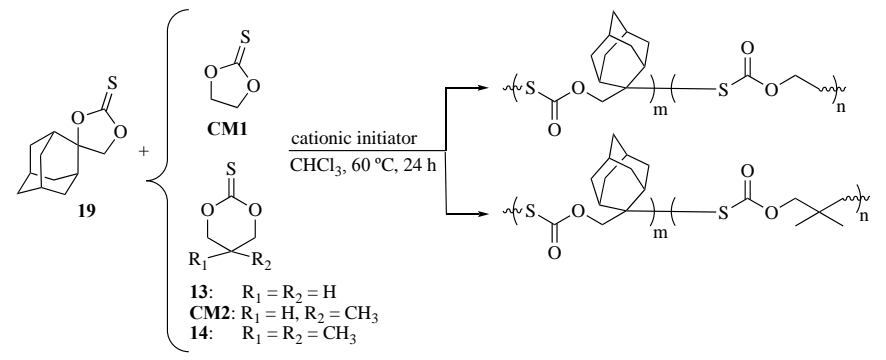

\begin{tabular}{|c|c|c|c|c|c|c|}
\hline entry & comonomer & initiator & yield $^{b}(\%)$ & $M_{n}{ }^{c}$ & $M_{\mathrm{w}} / M_{\mathrm{n}}{ }^{c}$ & $\begin{array}{c}\text { m:n unit ratio }{ }^{d} \\
(\mathrm{~mol} \%)\end{array}$ \\
\hline 1 & CM1 & $\mathrm{Et}_{3} \mathrm{OBF}_{4}$ & 22 & 2,100 & 1.40 & $37: 63$ \\
\hline 2 & CM1 & $\mathrm{BF}_{3} \mathrm{OEt}_{2}$ & 26 & 2,100 & 1.40 & $38: 62$ \\
\hline 3 & 13 & $\mathrm{Et}_{3} \mathrm{OBF}_{4}$ & 30 & 2,200 & 1.40 & $11: 89$ \\
\hline 4 & 13 & $\mathrm{BF}_{3} \mathrm{OEt}_{2}$ & 17 & 2,700 & 1.32 & $15: 85$ \\
\hline 5 & CM2 & $\mathrm{Et}_{3} \mathrm{OBF}_{4}$ & 10 & 3,000 & 1.57 & $25: 75$ \\
\hline 6 & CM2 & $\mathrm{BF}_{3} \mathrm{OEt}_{2}$ & 11 & 3,700 & 1.34 & $27: 73$ \\
\hline 7 & 14 & $\mathrm{Et}_{3} \mathrm{OBF}_{4}$ & 52 & 2,700 & 1.73 & $32: 68$ \\
\hline 8 & 14 & $\mathrm{BF}_{3} \mathrm{OEt}_{2}$ & 30 & 2,500 & 1.74 & $37: 63$ \\
\hline
\end{tabular}

Table 18. Cationic ring-opening copolymerization of 19 with $\mathrm{CM} 1, \mathrm{CM} 2, \mathbf{1 3}$, and 14 . $^{\text {a }}$ a Feed ratio of 19 with $\mathrm{CM} 1, \mathrm{CM} 2,13$, and 14, 1:1; solvent, $\mathrm{CHCl}_{3}$; monomer, $1 \mathrm{M}$; initiator, $2 \mathrm{~mol} \%$; temperature, $60 \stackrel{\circ}{\circ}$. ${ }^{b}$ Methanol insoluble portion. ${ }^{c}$ Estimated by GPC eluted with THF and based on polystyrene standards. ${ }^{d}$ The values of $m$ and $n$ were estimated from ${ }^{1} \mathrm{H} \mathrm{NMR}\left(\mathrm{CDCl}_{3}\right)$. 
To further the work of the Endo lab, Ochiai et al. studied the cationic ${ }^{65}$ and anionic ${ }^{66}$ ROP of a seven-membered cyclic monothiocarbonate, 1,3-dioxepan-2-thione (22). The authors successfully obtained polythiocarbonate by applying cationic catalysts such as $\mathrm{TfOH}$, TfOMe, $\mathrm{BF}_{3} \mathrm{OEt}_{2}$, and $\mathrm{BF}_{4} \mathrm{OEt}_{3}$, Table 19. The cationic polymerization proceeded in a linear fashion with respect to the feed ratio of initiator to monomer and the molecular weight distribution was moderate $(<1.30)$. Upon analysis of the products the authors suggest an isomerization

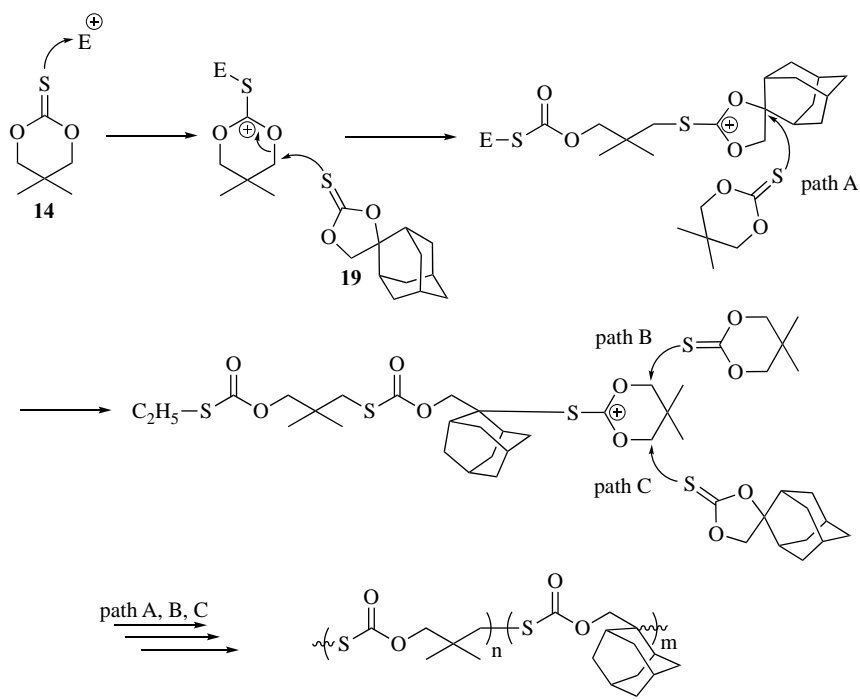

Scheme 11. Proposed mechanism for the copolymerization of $\mathbf{1 4}$ and $\mathbf{1 9 .}$

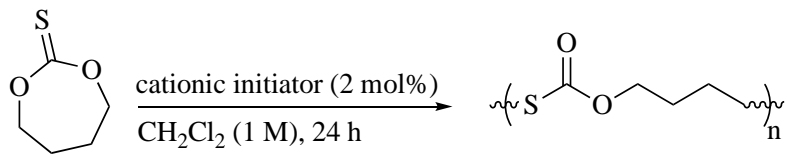

22

$\begin{array}{lcccccc}\text { entry } & \text { initiator } & T\left(^{\circ} \mathrm{C}\right) & \text { conv. }^{b}(\%) & \text { yield }^{c}(\%) & M_{\mathrm{n}}{ }^{d} & M_{\mathrm{w}} / M_{\mathrm{n}}{ }^{d} \\ 1 & \mathrm{TfOH} & 0 & 71 & 60 & 9,200 & 1.37 \\ 2 & \mathrm{TfOH} & 30 & >99 & 98 & 12,200 & 1.41 \\ 3 & \text { TfOMe } & 0 & 87 & 81 & 8,300 & 1.42 \\ 4 & \text { TfOMe } & 30 & >99 & 98 & 13,900 & 1.37 \\ 5 & \mathrm{BF}_{3} \mathrm{OEt} & 0 & >99 & 88 & 43,700 & 1.24 \\ 6 & \mathrm{BF}_{3} \mathrm{OEt}_{2} & 30 & >99 & 92 & 29,100 & 1.29\end{array}$


Table 19. Catalyst screen for the cationic ROP of 22. ${ }^{\text {a a }}$ Solvent, $\mathrm{CH}_{2} \mathrm{Cl}_{2} ;$ [22], $1 \mathrm{M}$; [initiator]/[22], 0.02 ; time, $24 \mathrm{~h} .{ }^{b}$ Determined by ${ }^{1} \mathrm{H}$ NMR $\left(\mathrm{CDCl}_{3}\right) .{ }^{c}$ Isolated yield after precipitation with $n$ hexane. ${ }^{d}$ Determined by GPC eluted with THF based on polystyrene standards.

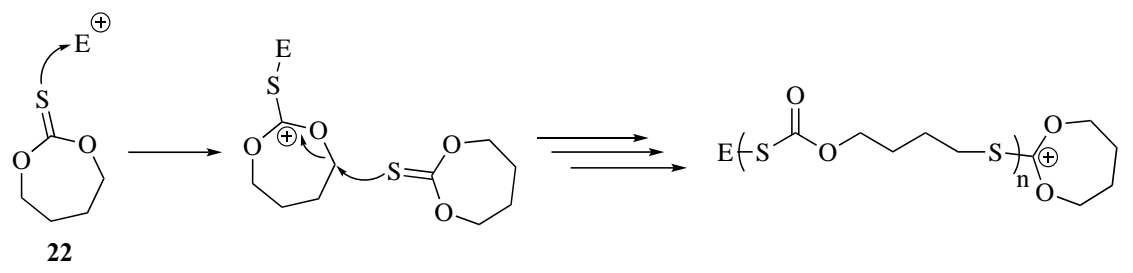

Scheme 12. Proposed isomerization mechanism for the cationic ROP of 22.

mechanism where the thiocarbonyl becomes a carbonyl species in the final product, Scheme 12. Ochiai et al. also applied anionic catalysts to the polymerization of $\mathbf{2 2}$, Table 20 . When the authors utilized lithium catalysts the polythiocarbonate products precipitated out of THF in a timely manner, Table 20 runs $1-4$. Upon analysis of the THF insoluble products produced from the lithium catalyzed anionic ROP the authors found 22 proceeds via a mechanism absent of isomerization and retains the thiocarbonyl functionality in the backbone of the polymer. However, when $t$-BuOK, TEA, pyridine, and DBU (Table 20 runs 5-8) were applied to the anionic ROP of 22 the authors noticed the products were soluble in THF. Through analysis, the polymers obtained from $t$-BuOK, TEA, and pyridine all appear to proceed via an isomerization pathway where the sulfur and oxygen switch places to yield a carbonyl species in the final product. In contrast, the DBU catalyzed anionic ROP of 22 appears to proceed either via isomerization of the thiocarbonyl to a carbonyl or through nucleophilic addition where the active species is the thiocarbonate anion; the authors suggest further studies in order to solidify which pathway is favored. The anionic ROP of 22 exhibits living characteristics; linear evolution of $\mathrm{Mn}$ with respect to the feed ratio, $[\mathbf{2 2}]_{0} /[t-\mathrm{BuOK}]_{0}$.

The future of ROP of thiol containing cyclic carbonates proves promising and exciting. With many possibilities, these monomers could prove extremely useful as copolymerization 
additives; increase tensile strength and optical properties. However, the work described in this manuscript is comprehensive, which means that there is much room to grow and many new discoveries to be made. When applied to S-containing carbonates, ROP proves to produce robust polymers in moderate to excellent yields, moderate molecular weights, and can even exhibit living characteristics. By adjusting the $\mathrm{M} / \mathrm{I}$ feed ratio, polymers of exact molecular weight are able to be synthesized.
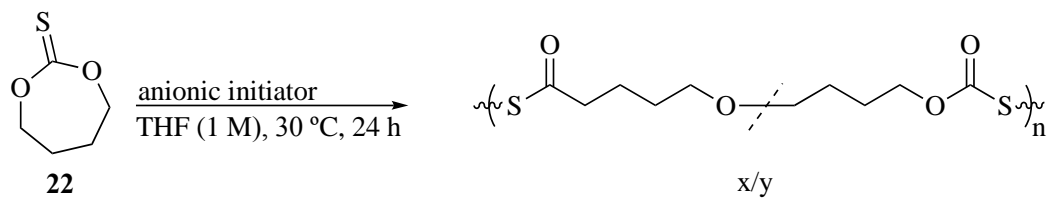

$\begin{array}{lcccccc}\text { entry } & \text { initiator } & \text { conv. }{ }^{b}(\%) & \text { yield }^{c}(\%) & M_{\mathrm{n}^{d}} & M_{\mathrm{w}} / M_{\mathrm{n}}{ }^{d} & \begin{array}{c}\text { unit ratio } \\ (\mathrm{x} / \mathrm{y})^{b}\end{array} \\ 1 & n \text {-BuLi } & >99 & 55^{d} & 2,400 & 1.08 & 100: 0 \\ 2 & s \text {-BuLi } & >99 & 57^{d} & 1,500 & 1.53 & 100: 0 \\ 3 & t \text {-BuLi } & >99 & 42^{d} & & \text { n.d. }^{f} & 100: 0 \\ 4 & t \text {-BuOLi } & >99 & 56^{d} & & \text { n.d. }{ }^{f} & 100: 0 \\ 5 & t \text {-BuOK } & >99 & 94^{e} & 8,800 & 1.07 & 0: 100 \\ 6 & \text { TEA } & >99 & 91^{e} & 50,400 & 1.56 & 0: 100 \\ 7 & \text { pyridine } & 68 & 72^{e} & 38,300 & 1.96 & 0: 100 \\ 8 & \text { DBU } & >99 & 98^{e} & 9,600 & 1.10 & 42: 58\end{array}$

Table 20. The anionic ROP of 22. ${ }^{a}$ a Solvent, THF; [22], $1 \mathrm{M}$; [initiator]/[22], 0.02 (runs 1-5) or 0.04 (runs 6-8); $30{ }^{\circ} \mathrm{C} ; 24 \mathrm{~h}$. ${ }^{b}$ Determined by ${ }^{1} \mathrm{H}$ NMR $\left(\mathrm{CDCl}_{3}\right)$. Determined from GPC eluted with THF based on polystyrene standards. ${ }^{d}$ Isolated yield after Soxhlet extraction with THF. ${ }^{e}$ Isolated yield after precipitation with $n$-hexane. ${ }^{f}$ Not determined. Dimer or trimer. 


\section{LIST OF REFERENCES}

(1) Fries, K.; Mengel, H. Berichte Dtsch. Chem. Ges. 1912, 45 (3), 3408-3411.

(2) Kricheldorf, H. R.; Schwarz, G. J. Macromol. Sci. Pure Appl. Chem. 2007, 44 (6), 625649.

(3) Goodrich, B. F. C. Improvements in the manufacture of thiolactones. BP840.658, July 6, 1960.

(4) Lin'kova, M. G.; Kil'disheva, O. V.; Knunyants, I. L. Izv Akad Nauke USSR Ser Khim 1955, 569 .

(5) Linkova, M. G.; Kildïsheva, G. V.; Knunyants, I. L. Bull. Acad. Sci. USSR Div. Chem. Sci. 1955, 4 (3), 507-508.

(6) Knunyants, I. L.; Kil'disheva, O. V.; Pervova, E. Y. Izv Akad Nauke USSR Ser Khim $1955,689$.

(7) Knunyants, I. L.; Kildisheva, O. V.; Pervova, E. Y. Bull. Acad. Sci. USSR Div. Chem. Sci. 1955, 4 (4), 613-618.

(8) Knunyants, I. L.; Pervova, E. Y.; Lin'kova, M. G.; Kil'disheva, O. V. Khim Nauke Promyshlenost 1958, 3, 278.

(9) Lin’kova, M. G.; Patrina, N. D.; Knunyants, I. L. Dokl Akad Nauk SSSR 1959, 127, 564.

(10) Lin'kova, M. G.; Patrina, N. D.; Knunyants, I. L. Izv Akad Nauk SSSR Ser Khim 1960, 1825.

(11) Knunyants, I. L.; Lin’kova, M. G.; Kuleshova, N. D. Izv Akad Nauk SSSR Ser Khim 1969, 4,644 .

(12) Knunyants, I. L.; Kuleshova, N. D.; Lin’kova, M. G. Izv Akad Nauk SSSR Ser Khim 1965, $6,1081$.

(13) Lin'kova, M. G.; Kuleshova, N. D.; Knunyants, I. L. Russ. Chem. Rev. 1964, 33 (10), 493.

(14) Kricheldorf, H. R. Makromol. Chem. 1973, 173 (1), 81-89.

(15) Overberger, C. G.; Weise, J. K. J. Am. Chem. Soc. 1968, 90 (13), 3533-3537.

(16) Schjånberg, E. Berichte Dtsch. Chem. Ges. B Ser. 1941, 74 (11), 1751-1759. 
(17) Korte, F.; Löhmer, K.-H. Chem. Ber. 1958, 91 (7), 1397-1403.

(18) Korte, F.; Büchel, K. H. Chem. Ber. 1960, 93 (5), 1021-1025.

(19) Heinz, K. A process for preparing thiolactones. DE859456C, December 15, 1952.

(20) Overberger, C. G.; Weise, J. J. Polym. Sci. B Polym. Lett. 1964, 2 (4), 329-331.

(21) Overberger, C. G.; Weise, J. K. J. Am. Chem. Soc. 1968, 90 (13), 3525-3532.

(22) Bannin, T. J.; Kiesewetter, M. K. Macromolecules 2015, 48 (16), 5481-5486.

(23) Brode, G.; Fritze, P. Preparation of thiolactones. US3732250 A, May 8, 1973.

(24) Fritze, P. Process for preparing epsilon-thiocaprolactones. US3786066 A, January 15, 1974.

(25) Fritze, P. Thiolactone polymerization and catalysts. US3755268 A, August 28, 1973.

(26) Matzner, M.; McGrath, J. E.; Chow, S. W.; Koleske, J. V.; Robeson, L. M. J. Appl. Polym. Sci. 1973, 17 (3), 983-986.

(27) Bhar, D.; Chandrasekaran, S. Tetrahedron 1997, 53 (34), 11835-11842.

(28) Kricheldorf, H. R.; Lee, S.-R.; Schittenhelm, N. Macromol. Chem. Phys. 1998, 199 (2), 273-282.

(29) Nederberg, F.; Connor, E. F.; Möller, M.; Glauser, T.; Hedrick, J. L. Angew. Chem. Int. Ed. 2001, 40 (14), 2712-2715.

(30) Nader, R. B.; Kaloustian, M. K. Tetrahedron Lett. 1979, 20 (17), 1477-1480.

(31) Kaloustian, M. K.; Khouri, F. Tetrahedron Lett. 1981, 22 (5), 413-416.

(32) Nicolaou, K. C.; McGarry, D. G.; Somers, P. K.; Kim, B. H.; Ogilvie, W. W.; Yiannikouros, G.; Prasad, C. V. C.; Veale, C. A.; Hark, R. R. J. Am. Chem. Soc. 1990, $112(17), 6263-6276$.

(33) Scheibye, S.; Kristensen, J.; Lawesson, S.-O. Tetrahedron 1979, 35 (11), 1339-1343.

(34) Cava, M. P.; Levinson, M. I. Tetrahedron 1985, 41 (22), 5061-5087.

(35) Curphey, T. J. Tetrahedron Lett. 2000, 41 (51), 9963-9966.

(36) Curphey, T. J. Tetrahedron Lett. 2002, 43 (3), 371-373.

(37) Curphey, T. J. J. Org. Chem. 2002, 67 (18), 6461-6473. 
(38) Curphey, T. J. Compositions and methods for thionation during chemical synthesis reactions. US20030176714A1, September 18, 2003.

(39) Filippi, J.-J.; Fernandez, X.; Lizzani-Cuvelier, L.; Loiseau, A.-M. Tetrahedron Lett. 2003, 44 (35), 6647-6650.

(40) Sanda, F.; Jirakanjana, D.; Hitomi, M.; Endo, T. Macromolecules 1999, 32 (24), 80108014.

(41) Sanda, F.; Jirakanjana, D.; Hitomi, M.; Endo, T. J. Polym. Sci. A Polym. Chem. 2000, 38 (22), 4057-4061.

(42) Kikuchi, H.; Tsubokawa, N.; Endo, T. Chem. Lett. 2005, 34 (3), 376-377.

(43) Datta, P. P.; Kiesewetter, M. K. Macromolecules 2016, 49 (3), 774-780.

(44) Braun, D.; Kiessel, M. Monatshefte Für Chem. Verwandte Teile Anderer Wiss. 1965, $96(2), 631-641$.

(45) Leung, L. M.; Chan, W. H.; Leung, S. K. J. Polym. Sci. A Polym. Chem. 1993, 31 (7), 1799-1806.

(46) Motokucho, S.; Takeuchi, D.; Sanda, F.; Endo, T. Tetrahedron 2001, 57 (33), 71497152.

(47) Soga, K.; Imamura, H.; Sato, M.; Ikeda, S. J. Polym. Sci. Polym. Chem. Ed. 1976, 14 (3), 677-684.

(48) Kihara, N.; Nakawaki, Y.; Endo, T. J. Org. Chem. 1995, 60 (2), 473-475.

(48) Choi, W.; Sanda, F.; Kihara, N.; Endo, T. J. Polym. Sci. A Polym. Chem. 1997, 35 (17), 3853-3856.

(50) Choi, W.; Sanda, F.; Endo, T. Macromolecules 1998, 31 (8), 2454-2460.

(51) Choi, W.; Sanda, F.; Endo, T. Macromolecules 1998, 31 (25), 9093-9095.

(52) Sanda, F.; Shinjo, T.; Choi, W.; Endo, T. Macromol. Rapid Commun. 2001, 22 (5), 363366.

(53) Endo, T.; Nagai, D. Macromol. Symp. 2005, 226 (1), 79-86.

(52) Steblyanko, A.; Choi, W.; Sanda, F.; Endo, T. J. Polym. Sci. A Polym. Chem. 2001, 39 (22), 3967-3980. 
(55) Soga, K.; Imamura, H.; Ikeda, S. Makromol. Chem. 1975, 176 (3), 807-811.

(56) Kricheldorf, H. R.; Damrau, D.-O. Macromol. Chem. Phys. 1998, 199 (11), 2589-2596.

(57) Nemoto, N.; Sanda, F.; Endo, T. Macromolecules 2000, 33 (20), 7229-7231.

(58) Endo, T.; Nemoto, N.; Sanda, F. Macromol. Symp. 2003, 192 (1), 25-30.

(59) Yonet, N.; Yagci, Y.; Ochiai, B.; Endo, T. Macromolecules 2003, 36 (24), 9257-9259.

(61) Nemoto, N.; Yoshii, K.; Kameshima, H.; Sanda, F.; Endo, T. J. Polym. Sci. A Polym. Chem. 2003, 41 (1), 185-195.

(61) Nemoto, N.; Xu, X.; Sanda, F.; Endo, T. Macromolecules 2001, 34 (22), 7642-7647.

(62) Kameshima, H.; Nemoto, N.; Sanda, F.; Endo, T. Macromolecules 2002, 35 (15), 57695773.

(64) Nemoto, N.; Ito, Y.; Endo, T. J. Polym. Sci. A Polym. Chem. 2003, 41 (5), 699-707.

(64) Kakimoto, K.; Nemoto, N.; Sanda, F.; Endo, T. Chem. Lett. 2002, 31 (2), 156-157.

(66) Ochiai, B.; Yoshii, K.; Nagai, D.; Endo, T. J. Polym. Sci. A Polym. Chem. 2005, 43 (5), 1014-1018.

(66) Ochiai, B.; Yoshii, K.; Nagai, D.; Endo, T. Macromolecules 2004, 37 (7), 2329-2331. 


\section{CHAPTER 2}

Published in Macromolecules, August 2015

Poly(thioester) by Organocatalytic Ring-Opening Polymerization

Timothy Bannin and Matthew Kiesewetter

Chemistry, University of Rhode Island, Kingston, RI USA

Corresponding Author: Matthew Kiesewetter, Ph.D.

Chemistry

University of Rhode Island

325C, Beaupre Hall, 140 Flagg Rd.

Kingston, RI, 02881, USA

Phone: +1-401-874-2619

Email address: mkiesewetter@chm.uri.edu 


\begin{abstract}
Organocatalysts typically used for the ring-opening polymerization (ROP) of cyclic ester monomers are applied to a thiolactone, $\varepsilon$-thiocaprolactone (tCL). In the absence of an $\mathrm{H}$-bond donor, a nucleophilic polymerization mechanism is proposed. Despite the decreased ability of thioesters and thiols (versus esters and alcohols) to $\mathrm{H}$-bond, $\mathrm{H}$-bonding organocatalysts a thiourea in combination with an $\mathrm{H}$-bond accepting base are also effective for the ROP of tCL. The increased nucleophilicity of thiols (versus alcohols) is implicated in the increased $M_{\mathrm{w}} / M_{\mathrm{n}}$ of the poly(thiocaprolactone) versus poly(caprolactone), but deleterious transesterification is suppressed in the presence of a thiourea. The thioester monomer, $\mathrm{tCL}$, is shown to be thermodynamically similar to $\varepsilon$-caprolactam but kinetically similar to $\varepsilon$-caprolactone.
\end{abstract}




\section{INTRODUCTION}

Organic catalysts for polymerization have provided efficient methods for the synthesis of welldefined, functionalized polymers. ${ }^{1,2}$ Cyclic esters and carbonates have been the most common monomers for organocatalytic ring-opening polymerization (ROP) methods; acrylates have also been employed. ${ }^{3-6}$ Expanding the scope of monomers available for organocatalytic ROP increases the diversity of materials and their applications. ${ }^{7,8}$ The increased nucleophilicity of thiols and altered electrophilicity of thioesters versus alcohols/esters make poly(thioester)s potentially attractive synthons for materials and a challenge for controlled ROP chemistry. The mild conditions of organocatalytic ROP provide a route to well defined poly(thioester)s. Sporadic entries to the literature concerning the ROP of $\mathrm{tCL}$ have appeared since the initial report in 1968..$^{9,10}$ Many reports feature late metal alkoxide (Sn, Cd, Mn, etc.) catalyzed ROP of $\mathrm{tCL}$ from alcohol or thiol initiators in solvent or bulk, ${ }^{11,12}$ and a ring-expansion polymerization technique has also been demonstrated. ${ }^{13}$ A recent report of the ROP of $\varepsilon$-thiocaprolactone, tCL, used a lipase typically employed in esterification ${ }^{14,15}$ to yield poly( $\varepsilon$-thionocaprolactone) (PtCL) with higher $M_{\mathrm{w}} / M_{\mathrm{n}}$ than poly( $\varepsilon$-caprolactone) (PCL) generated under identical conditions. This report demonstrates the extension of mild techniques for the ROP of esters to thioesters. Herein, we disclose the "living" ROP of tCL using organocatalysts; the application of thiourea $\mathrm{H}$-bond donors is discussed and a polymerization mechanism is proposed. 


\section{RESULTS AND DISCUSSION}

Polymerization Thermodynamics. The first reports by Overberger and Weise in $1968^{9,10}$ suggested that strong base organocatalysts may be effective for the ROP of tCL; these reports demonstrated that strong alkoxide and alkyl-lithium bases effect the ROP of tCL in the bulk. ${ }^{9}$ The reported polymerizations were uncontrolled, and access to molecular weight/dispersity information was limited. The effectiveness of strong alkoxide bases for ROP of tCL suggested that the strong base and potent transesterification agent, 1,5,7-triazabicyclo[4.4.0]dec-5-ene (TBD), ${ }^{16}$ might also be effective for the ROP of tCL. Indeed, the introduction of TBD (5 mol\%) into a $\mathrm{CDCl}_{3}$ solution of $\mathrm{tCL}(1 \mathrm{M})$ and octadecylthiol $(2 \mathrm{~mol} \%)$ results in full conversion to polymer in $30 \mathrm{~s}\left(M_{\mathrm{n}}=6000 \mathrm{~g} / \mathrm{mol} ; M_{\mathrm{w}} / M_{\mathrm{n}}=1.7\right)$. If the reaction is not quenched, the $M_{\mathrm{w}} / M_{\mathrm{n}}$ rapidly broadens post polymerization, and timing the quench of this rapid reaction is difficult.

As opposed to cyclic lactones, only the 7-membered thiolactone, $\varepsilon$-thiocaprolactone (tCL), is thought to be thermodynamically favored to undergo ROP. ${ }^{9}$ However, the magnitude of the thermodynamic driving force has not been reported, but we were able to employ the rapid TBDcatalyzed ROP of $\mathrm{tCL}$ to measure the thermodynamics of polymerization. The equilibrium monomer concentration of a solution of tCL (1 M), octadecylthiol (2 mol \%) and TBD (20 mol \%) in $\mathrm{CDCl}_{3}$ was measured versus temperature, ${ }^{17}$ and the resulting Van' $\mathrm{t}$ Hoff analysis yielded the thermodynamics of ROP for tCL: $\Delta \mathrm{H}_{\mathrm{p}}^{0}=-2.43 \pm 0.69 \mathrm{kcal} / \mathrm{mol} ; \Delta \mathrm{S}_{\mathrm{p}}{ }_{\mathrm{p}}=-0.35 \pm 0.22 \mathrm{cal} / \mathrm{mol}$. $\mathrm{K} ;[\mathrm{M}]_{\mathrm{eq}}=0.018$ at $293 \mathrm{~K}$ and $\mathrm{T}_{\mathrm{c}}=7,000 \mathrm{~K}$. This data describes a polymerization reaction that highly favors polymer and suggests that $\mathrm{tCL}$ is energetically more similar to caprolactam (no ceiling temperature) than it is $\varepsilon$-caprolactone $(\mathrm{CL})$ or $\delta$-valerolactone $(\mathrm{VL})\left(\mathrm{T}_{\mathrm{c}} \sim 534 \mathrm{~K}\right.$ and $\mathrm{T}_{\mathrm{c}} \sim$ $422 \mathrm{~K}$, respectively). ${ }^{17}$ 


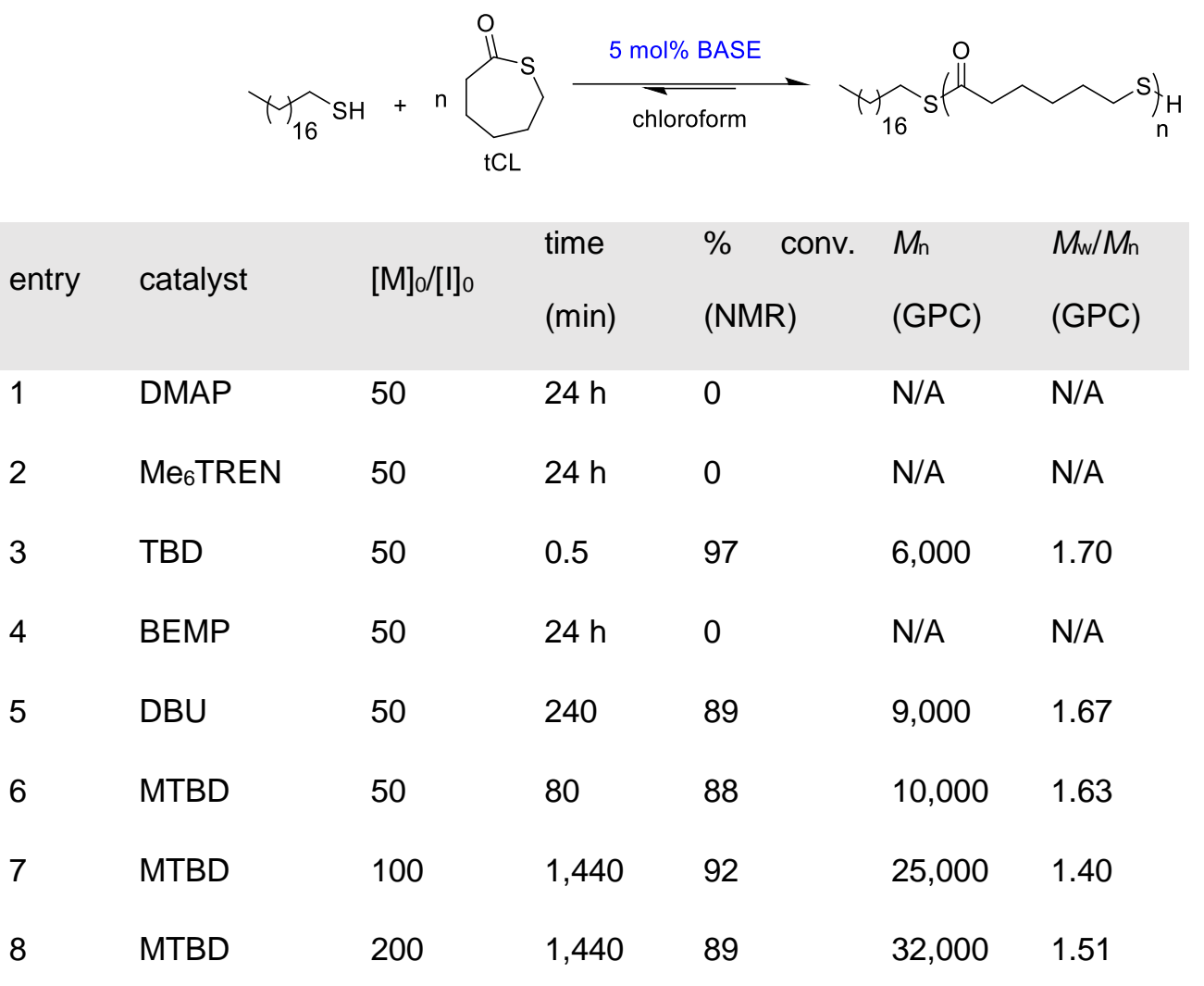

Table 1. Catalyst Screen for the Ring-Opening Polymerization if tCL. Reaction conditions: 100 $\mathrm{mg}(0.77 \mathrm{mmol}, 1 \mathrm{M}) \mathrm{tCL} ; 0.015 \mathrm{mmol}$ octadecylthiol, $0.038 \mathrm{mmol}$ base catalyst in $\mathrm{CHCl}_{3}$ (BEMP reaction was attempted in both $\mathrm{CDCl}_{3}$ and $\mathrm{C}_{6} \mathrm{D}_{6}$ ).

Organic Base Catalyzed ROP. A screen of base catalysts revealed that only strong, nucleophilic bases are active for the ROP of tCL. The addition of $5 \mathrm{~mol} \%$ (to monomer) base catalyst to a $\mathrm{CDCl}_{3}$ solution of $\mathrm{tCL}(1 \mathrm{M})$ and octadecylthiol $(2 \mathrm{~mol} \%)$ resulted in ROP only for amidine bases. MTBD (7-methyl-1,5,7-triazabicyclo[4.4.0]dec-5-ene; MTBD- $\mathrm{H}^{+} \mathrm{pK}_{\mathrm{a}}{ }^{\mathrm{MeCN}}=$ $25.4)^{18}$ and DBU (1,8-diazabicyclo[5.4.0]undec-7-ene; $\left.\mathrm{DBU}-\mathrm{H}^{+} \mathrm{pK}_{\mathrm{a}}^{\mathrm{MeCN}}=24.3\right)^{18}$ resulted in full consumption of monomer in a reasonable time scale, while tris[2-(dimethylamino)ethyl]-amine (Me6 6 TREN), BEMP (2-tert-butylimino-2-diethylamino-1,3-dimethylperhydro-1,3,2diazaphosphorine; BEMP- $\left.\mathrm{H}^{+} \mathrm{pK}_{\mathrm{a}}^{\mathrm{MeCN}}=27.6\right)^{19}$ and DMAP (4-(dimethylamino)pyridine; $\left.\mathrm{DMAP}-\mathrm{H}^{+} \mathrm{pK}_{\mathrm{a}}=18.2\right)^{20}$ resulted in no observable conversion to polymer, Table 1. Poly(thiocaprolactone) exhibits good solubility in chlorinated solvents but is minimally soluble in THF. 
The high activity of DBU and MTBD for the ROP of tCL combined with the observation that the considerably more basic but non-nucleophilic BEMP did not form polymer suggests a nucleophilic ROP mechanism. As shown in Table 1, both amidine bases provided rapid but controllable ROP and moderate $M_{w} / M_{n}\left(\mathrm{DBU}, M_{w} / M_{n}=1.67 ; \mathrm{MTBD}, M_{\mathrm{w}} / M_{\mathrm{n}}=1.63\right)$. For the MTBD and DBU catalyzed ROPs, the evolution of $M_{\mathrm{n}}$ versus conversion was linear (Figure 1), $M_{\mathrm{w}} / M_{\mathrm{n}}$ remained low but broadened with increased reaction time, and $M_{\mathrm{n}}$ is predictable from $[M]_{0} /[]_{0}$, Table 1 entries $6-8$. Poly tCL becomes insoluble in chlorinated solvents at high degree of polymerization ( $D P \geq 200$ ). Kinetic analyses reveal first order consumption of monomer versus time for the MTBD or DBU catalyzed ROPs (see Supporting Information Figures S1 and S2). These data suggest that MTBD and DBU exhibit the characteristics of a "living"
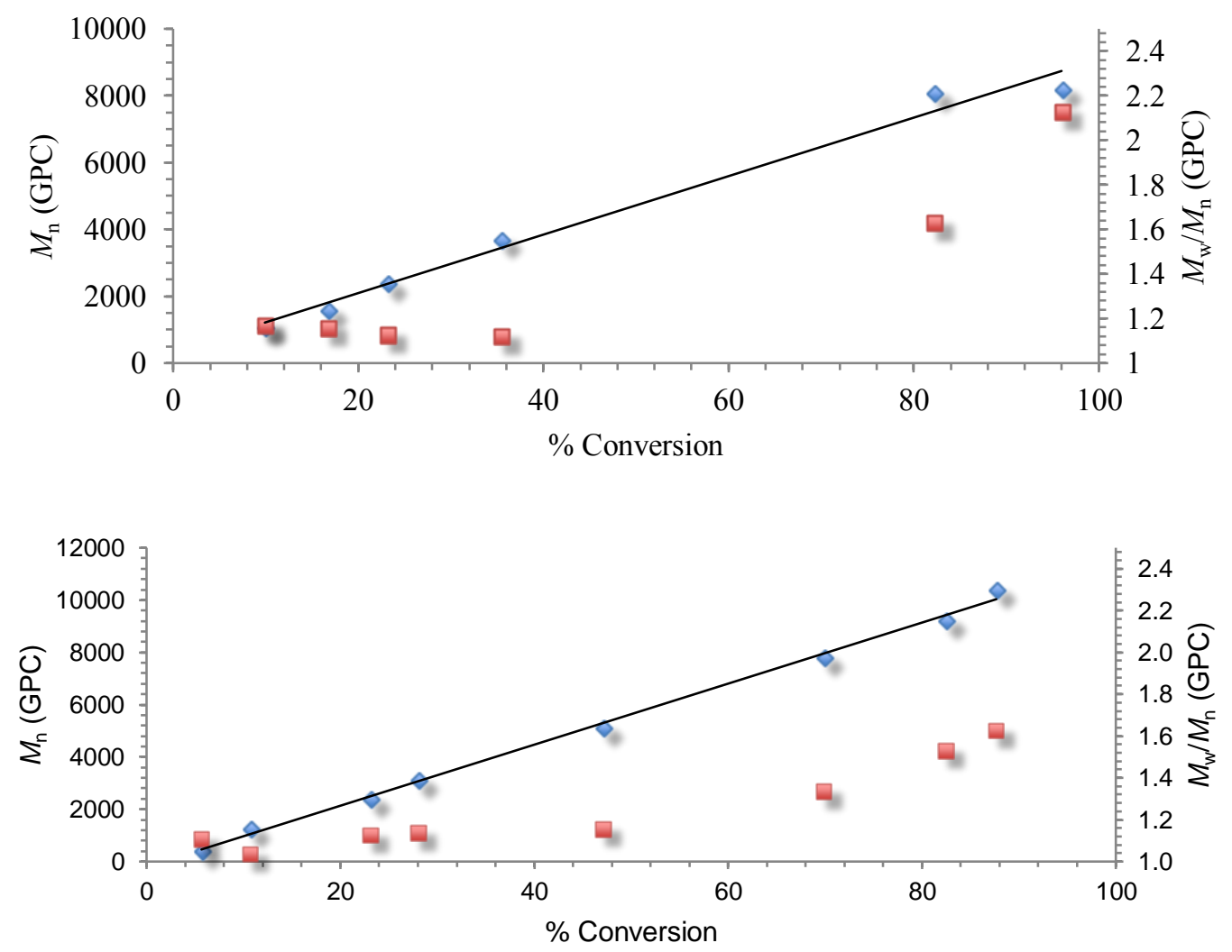

Figure 1. Evolution of percent conversion vs $M_{\mathrm{n}}$ (blue) and $M_{\mathrm{w}} / M_{\mathrm{n}}$ (red) for the ROP of tCL (1M) from octadecylthiol $(0.02 \mathrm{M})$ in chloroform catalyzed by (upper) $0.05 \mathrm{M} \mathrm{MTBD}$; and (lower) 0.05 M MTBD and 0.05 M 1. Conversion determined by NMR. 
polymerization while the relatively high $M_{\mathrm{w}} / M_{\mathrm{n}}$ (vs polyesters) may be attributable to the increased nucleophilicity of thiols versus alcohols. The surprising observation that the strongest and bulkiest Brønsted base examined (BEMP) is inoperative for ROP suggests that DBU and MTBD are not acting as general bases but rather are effecting ROP via nucleophilic attack at the thioester moiety, Scheme 1. Under basic conditions, thioesters are expected to

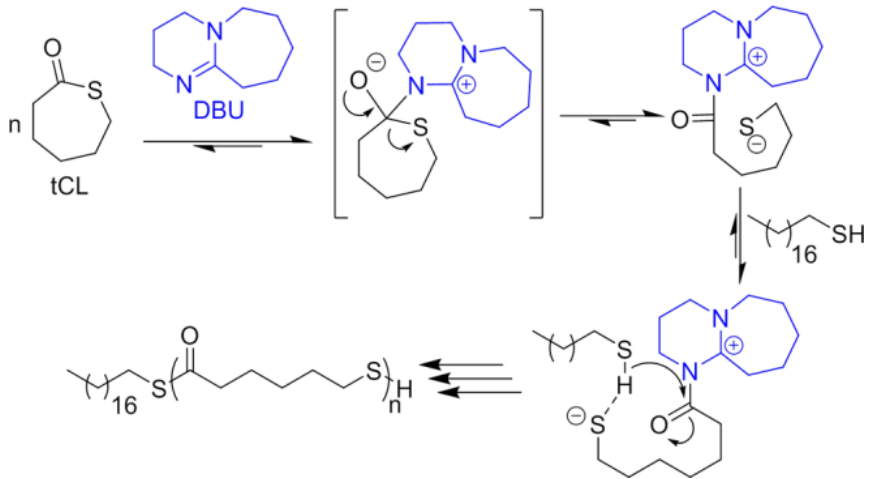

Scheme 1. Nucleophilic Mechanism for the ROP of tCL with DBU.

be better electrophiles than esters, ${ }^{5}$ which may account for the different reactivity vs organocatalytic ROP of esters, but nucleophilic modes of action have previously been suggested for these amidine bases. ${ }^{21}$

Effect of Thiourea upon Catalysis. The perturbation to ring geometry that occurs upon the change from caprolactone to thiocaprolactone was expected to render thiourea $\mathrm{H}$-bond donors ineffective for the activation of tCL. An NMR titration study in $\mathrm{C}_{6} \mathrm{D}_{6}$ was conducted to determine

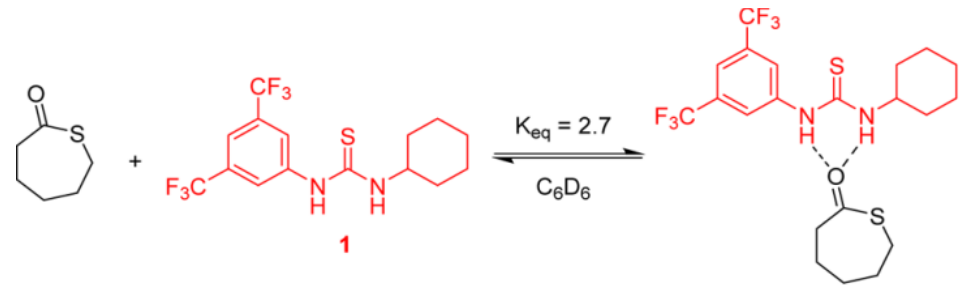

Equation 1. Binding between $\mathrm{tCL}$ and $\mathbf{1 .}$

the binding constant between 1 (in eq 1) and tCL, eq 1, $\mathrm{K}_{\text {eq }}=2.7 \pm 0.5$. The analogous binding constant between $\mathrm{CL}$ and 1 was reported to be $\mathrm{K}_{\mathrm{eq}}=42 .{ }^{16}$ DFT-predicted geometries for $\mathrm{CL}$ and tCL (see Supporting Information Figure S3) support the NMR binding studies. The dipole 
of $\mathrm{CL}$, which is activated by $1,{ }^{16}$ is aligned with the carbonyl whereas that of $\mathrm{tCL}$ is off set, which corroborates the observed minimal activation of $\mathrm{tCL}$ by 1 . Despite the small binding constant between $\mathrm{tCL}$ and 1, the H-bond donor exhibits a marked effect upon the ROP. The addition of an equimolar amount of $\mathbf{1}$ (to base) in the DBU catalyzed ROP of tCL from octadecylthiol decreases the reaction time (240 min versus $120 \mathrm{~min}$ ) and lowers $M_{\mathrm{w}} / M_{\mathrm{n}}$ (1.67 versus 1.47). For the analogous MTBD catalyzed experiment, the addition of TU has no effect on the rate, but the $M_{\mathrm{w}} / M_{\mathrm{n}}$ is lower in the presence of 1 (1.83 versus 1.63$)$. These results corroborate a previous report from our laboratory which suggested that the selectivity of 1 /base cocatalyzed ROP is due, in part, to favorable interactions between base and $1 .{ }^{22}$ The increased rate of the DBU experiment in the presence of 1 suggests that some monomer activation by TU may be operative despite the low binding constant, eq 1 . The evolution of $M_{\mathrm{n}}$ vs conversion plots for the MTBD or DBU plus 1 catalyzed ROP of $\mathrm{tCL}$ are linear which suggests a "living" ROP, Figure 1 and Supporting Information Figure S4, respectively. The $M_{\mathrm{w}} / M_{\mathrm{n}}$ versus conversion plots demonstrate that transesterification at high conversion (especially past $50 \%$ conversion) leads to broadened $M_{\mathrm{w}} / M_{\mathrm{n}}$, but this broadening is suppressed versus those ROPs in the absence of TU (see Supporting Information Figures S1 and S2). When initiated from 1-pyrenebutanol (2 $\mathrm{mol} \%$ ), the ROP of $\mathrm{tCL}(1 \mathrm{M})$ catalyzed by MTBD/1 (5 mol \% each) in $\mathrm{CHCl}_{3}$ exhibits similar ring-opening kinetics as when initiated from octadecylthiol, and the resulting polymer exhibits overlapping RI and UV GPC traces $\left(M_{n}=21000 \mathrm{~g} / \mathrm{mol} ; M_{\mathrm{w}} / M_{\mathrm{n}}=2.11\right)$, see Supporting Information Figure S5. These observations suggest end group fidelity and "living" ROP behavior.

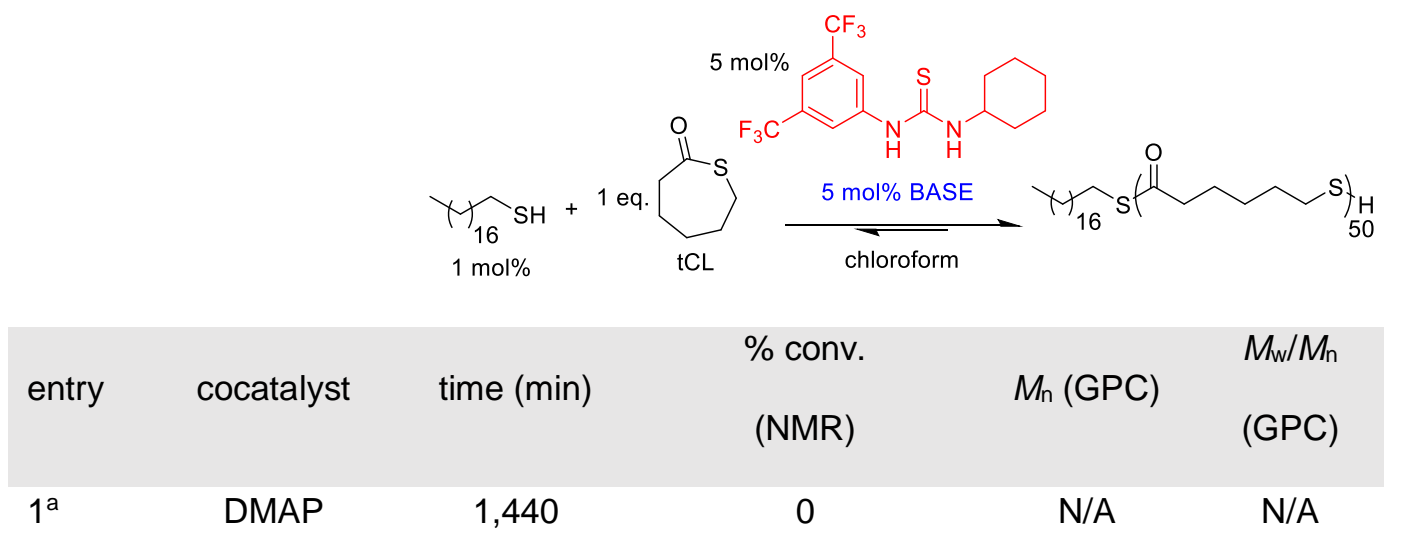




$\begin{array}{rrrrrr}2^{\mathrm{a}} & \text { Me } 6 \text { TREN } & 1,440 & 0 & \mathrm{~N} / \mathrm{A} & \mathrm{N} / \mathrm{A} \\ 3 & \text { BEMP } & 960 & 100 & 10,000 & 1.45 \\ 4 & \text { DBU } & 120 & 88 & 9,000 & 1.47 \\ 5 & \text { MTBD } & 80 & 88 & 10,000 & 1.63\end{array}$

Table 2. Base Catalyzed ROP of $\mathrm{tCL}$ in the Presence of Thiourea 1. Reaction conditions: 100 $\mathrm{mg}(0.77 \mathrm{mmol}, 1 \mathrm{M})$ of tCL, $0.015 \mathrm{mmol}$ of octadecylthiol, $0.038 \mathrm{mmol}$ of base, $0.038 \mathrm{mmol}$ of 1 in $\mathrm{CHCl}_{3}$. ${ }^{a}$ Reaction did not convert in 24 hours.

The mechanism of ROP (Table 2) is altered in the presence of 1 . Though inactive when alone, BEMP is observed to co-catalyze the formation of polymer when applied with $\mathbf{1}$ in the ROP of tCL. Concentration dependent ${ }^{1} \mathrm{H}$ NMR spectra of BEMP and octadecylthiol implicate a chainend activating role for BEMP in a bifunctional BEMP/1 catalyzed ROP of $\mathrm{tCL}$. In an equimolar mixture of BEMP and octadecylthiol (10 mM each) in $\mathrm{C}_{6} \mathrm{D}_{6}$, the chemical shifts of all resonances are negligibly altered in the presence vs absence of the other species, which suggests that quantitative deprotonation of the thiol is not occurring despite the strong basicity of BEMP. However, concentrating the mixture results in thiol proton exchange as evidenced by the broadening of the thiol $\mathrm{H}$ and $\alpha$-methylene resonances due to increased decoherence of this coupling constant at high concentration. The $\mathrm{J}^{3} \mathrm{HH}$ coupling between those protons is eventually lost at $100 \mathrm{mM}$ in each species. The same phenomena are observed when MTBD or DBU are used instead of BEMP, but this phenomenon is not observed in a solution of octadecylthiol alone. Thiols are generally weaker $\mathrm{H}$-bond donors than alcohols, ${ }^{23}$ and while BEMP cannot be observed to $\mathrm{H}$-bond to the thiol (no chemical shift), its presence is sufficient to cause rapid chemical exchange. These observations are consistent with a chain-end activation mode of action where BEMP is activating the thiol proton for nucleophilic attack, Scheme 2. This is in contrast to traditional poly(ester) organocatalysis wherein the chain-end is activated through strong $\mathrm{H}$-bonding. 


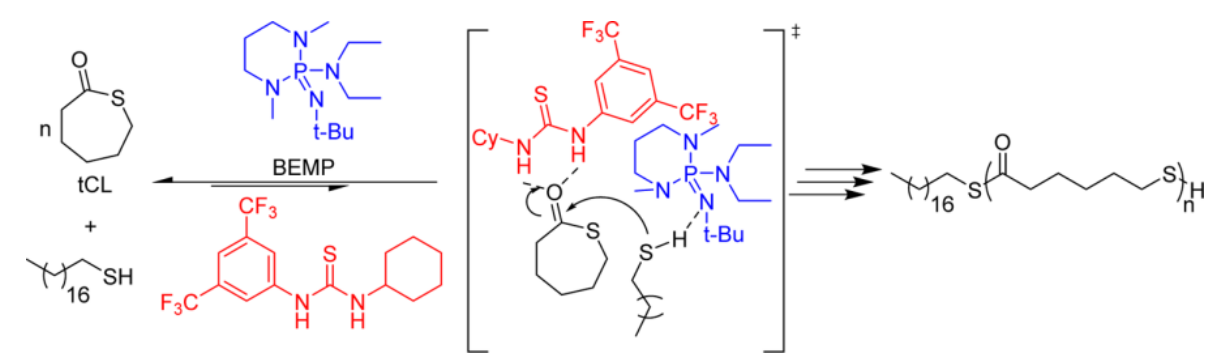

Scheme 2. Proposed Bifunctional Mechanism for the ROP of tCL by BEMP/1 Cocatalysts.

Thiocaprolactone vs Lactone Monomers. The kinetic behavior of tCL is unusual vis-à-vis ester monomers which demonstrate relative ring-opening kinetics: $k_{L A}>k_{V L} \gg k_{C L}$, where $L A$ is lactide. Typically, those monomers which are kinetically reluctant to open $(C L)$ require strong bases (higher $\mathrm{pK}_{\mathrm{a}}$ ) in conjunction with an $\mathrm{H}$-bond donor (1) to effect ROP.,2,16 Kinetically facile ROPs (like those with LA) will require only strong bases (MTBD, DBU, TBD, etc.), but these ROPs are generally far more controlled upon the application of a weak base (e.g., Me6 TREN) in conjunction with $1 .^{24,25}$ In this broader context of ester monomers, tCL occupies an unusual space in that it demonstrates ROP behavior that is both more and less reactive than VL. The thiolactone is more reactive in that it opens upon the application of strong base (i.e., DBU, MTBD) alone, which may be attributed to the increased nucleophilicity of thiols vs alcohols. It is less reactive in that upon the application of strong base and $\mathbf{1}$, its rate of ROP is slower when compared to the same reaction with VL. ${ }^{22}$ This observation could be due to the decreased ability of $\mathbf{1}$ to activate thioesters vs esters or the reduced electrophilicity of the thioester moiety. 


\section{CONCLUSION}

The organocatalytic ROP of tCL exhibits the characteristics of a "living" polymerization. Typical ester organocatalytic ROP results in extremely narrow $M_{\mathrm{w}} / M_{\mathrm{n}}$ which is eroded in the case of the ROP of $\mathrm{tCL}$ late in the reaction. This phenomenon may be attributable to the increased nucleophilicity of thiols (versus alcohols). The extremely rapid rate of the TBD-catalyzed ROP and the rate acceleration observed upon the addition of $\mathrm{H}$-bond donor $\mathbf{1}$ to the base (DBU, MTBD or BEMP) catalyzed ROP suggest that thioester activation of $\mathrm{tCL}$ may contribute to the accelerated ROP of $\mathrm{tCL}$. If this is the case, the binding between $\mathrm{tCL}$ and $\mathbf{1}$ would be among the weakest observed to effect catalysis. The suppression of $M_{w} / M_{n}$ broadening upon the addition of TU may be attributable to the strong interaction of $\mathbf{1}$ and amine base catalysts, as previously described. ${ }^{22}$ The decreased $\mathrm{H}$-bonding ability of thiols (vs alcohols) and the altered electrophilicity of thioesters (vs esters) dominates the ROP of poly(thiocaprolactone), but the collective effects of extraordinarily weak bifunctional activation by 1 and strong base serve to effect the ROP of $\mathrm{tCL}$. We expect that the incorporation of this new polymer backbone into the lexicon of organocatalytic ROP will facilitate the generation of new materials and applications. 


\section{EXPERIMENTAL SECTION}

General Considerations. All chemicals were purchased from Fisher Scientific except where indicated: 6-Bromohexanoic acid (Chem-Impex International, Inc.), sodium hydrosulfide monohydrate (Sigma-Aldrich), 1-octadecanethiol (Sigma-Aldrich). All chemicals were used as received except where indicated. HPLC grade methylene chloride (DCM) and tetrahydrofuran (THF) were dried on an Innovative Technology solvent system featuring alumina columns. Chloroform and chloroform-d (Cambridge Isotopes) were distilled from calcium hydride $\left(\mathrm{CaH}_{2}\right)$ under vacuum (10 mTorr), stored over $4 \AA$ molecular sieves, and passed through a plug of activated basic alumina just before use. Benzene- $d_{6}$ (Cambridge) was distilled from $\mathrm{CaH}_{2}$ under nitrogen atmosphere and stored over $3 \AA$ sieves. 1-[3,5-Bis(trifluoromethyl)phenyl]-3cyclohexylthiourea (1) was prepared according to literature procedures. ${ }^{16}$ All reactions were performed in a glovebox or by standard Schlenk techniques under $\mathrm{N}_{2}$ atmosphere and at room temperature, unless stated otherwise. ${ }^{1} \mathrm{H}$ and ${ }^{13} \mathrm{C}$ NMR spectra were obtained utilizing a Bruker Avance III 300 instrument at 300 and $75 \mathrm{MHz}$, respectively. Gel permeation chromatography (GPC) was performed in DCM utilizing an Agilent Technologies 1260 Infinity fitted with three 5 $\mu \mathrm{m}$ Agilent analytical columns connected in series with increasing pore size $\left(10^{5}, 10^{4}, 10^{3} \AA\right)$, an Agilent Infinity 1260 refractive index detector, and an Agilent Infinity 1260 UV/vis detector (250 and $300 \mathrm{~nm}$ ), calibrated with polystyrene standards. DFT calculations were run with Spartan '14 at the DFT B3LYP/6-31G* level of theory, gas phase.

Preparation of 6-Mercaptohexanoic Acid. A 1 L round-bottom flask was charged with 6bromohexanioc acid $(10 \mathrm{~g}, 51.3 \mathrm{mmol}), \mathrm{MeOH}(500 \mathrm{~mL})$, and a magnetic stir bar. After the 6bromohexanoic acid dissolved, sodium hydrosulfide monohydrate $(11.4 \mathrm{~g}, 154 \mathrm{mmol})$ was added, placed onto a hot/stir plate, and refluxed under a stream of $\mathrm{N}_{2}$ for $24 \mathrm{~h}$. After $24 \mathrm{~h}$, the reaction was removed from the heat and cooled to room temperature under $N_{2}$. The reaction mixture was then acidified with $\mathrm{H}_{2} \mathrm{SO}_{4}(\mathrm{pH}=5)$. Next, DI water was added to mixture $(\sim 50 \mathrm{~mL})$ and extracted three times with DCM. Organics were dried with $\mathrm{MgSO}_{4}$, and all volatiles were removed in vacuo to yield a colorless oil ( $6.67 \mathrm{~g}, 88 \%$ yield). Crude material was carried forward without purification; characterization matched the literature. ${ }^{26}{ }^{1} \mathrm{H} \mathrm{NMR}\left(\mathrm{CDCl}_{3}\right): \delta=2.57-2.49$ 
(q, $2 \mathrm{H} ;-\mathrm{CH}_{2} \mathrm{SH}$ ), 2.39-2.34 (t, $\left.2 \mathrm{H} ;-\mathrm{CH}_{2} \mathrm{COOH}\right), 1.70-1.59\left(\mathrm{~m}, 2 \mathrm{H} ;-\mathrm{CH}_{2} \mathrm{CH}_{2} \mathrm{SH}\right), 1.50-1.42$ (m, $\left.2 \mathrm{H} ; \mathrm{CH}_{2} \mathrm{CH}_{2} \mathrm{COOH}\right), 1.37-1.31\left(\mathrm{~m}, 2 \mathrm{H} ;-\mathrm{CH}_{2}\left(\mathrm{CH}_{2}\right)_{2} \mathrm{SH}\right)$.

Preparation of $\varepsilon-\mathrm{tCL}$. A dried $25 \mathrm{~mL}$ round-bottom flask was charged with 6-mercaptohexanoic acid $(7.00 \mathrm{~g}, 0.0472 \mathrm{mmol})$, phosphorus pentoxide $(4.022 \mathrm{~g}, 0.0283 \mathrm{mmol})$, and a stir bar. The flask was attached to a short path distillation head fitted with a receiving flask which had both been baked overnight at $140{ }^{\circ} \mathrm{C}$, and the apparatus was allowed to cool under $\mathrm{N}_{2}$ for approximately $20 \mathrm{~min}$. Once cooled, the apparatus was subjected to high active vacuum. After $5 \mathrm{~min}$, the pressure had reached $10 \mathrm{~mm} \mathrm{Hg}$, and the distilling flask was heated to $200{ }^{\circ} \mathrm{C}$. The receiving flask was placed into an ice bath. After approximately $1 \mathrm{~h}$, the distillation head was at room temperature, and the temperature of the reaction flask was increased $\left(210^{\circ} \mathrm{C}\right)$ and left to react until the distillation head was again at room temperature. This process was repeated once more at $220^{\circ} \mathrm{C}$. The apparatus was removed from the heat and allowed to cool under $\mathrm{N}_{2}$ until it reached room temperature. The yellow-orange oil was then purified via silica gel column chromatography (90:10 hexanes:ethyl acetate) and further purified via Kugelrohr distillation (50 $\left.{ }^{\circ} \mathrm{C}, 200 \mathrm{mTorr}\right)$ which yielded a colorless, odorless oil (1.5 g). The characterization matched the literature (see Supporting Information Figure S6 - S8). ${ }^{1} \mathrm{H} \mathrm{NMR}\left(\mathrm{CDCl}_{3}\right): \delta=3.05-3.01(\mathrm{t}$, $\left.2 \mathrm{H} ;-\mathrm{CH}_{2} \mathrm{SC}(\mathrm{O})-\right), 2.88-2.84$ (t, $\left.2 \mathrm{H} ;-\mathrm{CH}_{2} \mathrm{C}(\mathrm{O}) \mathrm{S}-\right)$, 2.16-2.09 (m, 2H; $\left.-\mathrm{CH}_{2} \mathrm{CH}_{2} \mathrm{~S}-\right)$, 1.88-1.74 (m, 4H; $\left.\left.-\mathrm{CH}_{2}\right)_{2} \mathrm{CH}_{2} \mathrm{C}(\mathrm{O})-\right) .{ }^{13} \mathrm{CNMR}\left(\mathrm{CDCl}_{3}\right): \delta=207.11$ (s, 1C, $\left.-\mathrm{SC}(\mathrm{O}) \mathrm{CH}_{2}-\right), 45.87(\mathrm{~s}, 1 \mathrm{C}$, $\left.-\mathrm{C}(\mathrm{O}) \mathrm{CH}_{2}\right) \quad 31.76$ (s, $\left.1 \mathrm{C},-\mathrm{SCH}_{2}-\right), 31.50$ (s, 1C, $\left.-\mathrm{SCH}_{2} \mathrm{CH}_{2}-\right), 30.90$ (s, 1C, $-\mathrm{C}(\mathrm{O}) \mathrm{CH}_{2} \mathrm{CH}_{2} \mathrm{CH}_{2}-$ ), 23.42 (s, $1 \mathrm{C},-\mathrm{C}(\mathrm{O}) \mathrm{CH}_{2} \mathrm{CH}_{2}-$ ). GC-MS (electron ionization): $\mathrm{m} / \mathrm{z}=130.1$ $\mathrm{g} \mathrm{mol}^{-1} ;$ mass $=130.05 \mathrm{~g} \mathrm{~mol}^{-1}$.

Representative Polymerization of $\varepsilon$-tCL with DBU and 1. $\varepsilon$-tCL (100 mg, $0.768 \mathrm{mmol},[1 \mathrm{M}])$ was dissolved in half of the total $\mathrm{CHCl}_{3}(0.77 \mathrm{~mL})$ used in the reaction and added to a solution of 1-octadecanethiol (4.4 mg, $0.015 \mathrm{mmol}), 1$ (14.2 mg, $0.038 \mathrm{mmol})$, and DBU (5.9 mg, 0.038 mmol) made with the remaining $\mathrm{CHCl}_{3}$. The reaction was left to stir for $180 \mathrm{~min}$, quenched with benzoic acid $(3.0 \mathrm{mg})$, and solvent removed in vacuo to yield a white film. Conversion was determined by NMR and polymer purified by precipitation from DCM with hexanes. ${ }^{1} \mathrm{H}$ NMR $\left(\mathrm{CDCl}_{3}\right): \delta=3.53-3.49\left(\mathrm{t}, 2 \mathrm{H} ;\left(\mathrm{CH}_{2}\right){ }_{16} \mathrm{CH}_{2} \mathrm{~S}\right), 2.87-2.82\left(\mathrm{t}, \sim 66 \mathrm{H} ; \mathrm{PB} \mathrm{CH}_{2} \mathrm{~S}\right), 2.55-2.50(\mathrm{t}, \sim 58 \mathrm{H}$; 
PB C $\left.(\mathrm{O}) \mathrm{CH}_{2}\right), 1.71-1.52(\mathrm{~m}, \sim 128 \mathrm{H} ; \mathrm{PB} \mathrm{CH} 2), 1.43-1.33\left(\mathrm{~m}, \sim 61 \mathrm{H} ; \mathrm{PB} \mathrm{CH}_{2}\right), 0.89-0.85$ (t, 3H; $\mathrm{CH}_{3} \mathrm{CH}_{2}$ ). 13C NMR $\left(\mathrm{CDCl}_{3}\right): \delta=199.29$ (s, 50C, C(O)CH$\left.{ }_{2}^{-}\right), 43.86\left(\mathrm{~s}, 50 \mathrm{C},-\mathrm{C}(\mathrm{O}) \mathrm{CH}_{2}\right) 29.29$ (s, 50C, $-\mathrm{SCH}_{2}{ }^{-}$), 28.53 (s, 50C, $-\mathrm{SCH}_{2} \mathrm{CH}_{2}{ }^{-}$), 28.11 (s, 50C, $-\mathrm{C}(\mathrm{O}) \mathrm{CH}_{2} \mathrm{CH}_{2} \mathrm{CH}_{2}{ }^{-}$), 25.12 (s, $\left.50 \mathrm{C},-\mathrm{C}(\mathrm{O}) \mathrm{CH}_{2} \mathrm{CH}_{2}-\right)$. GPC (UV-vis): $M_{\mathrm{n}}\left(M_{\mathrm{w}} / M_{\mathrm{n}}\right)=8300 \mathrm{~g} \mathrm{~mol}^{-1}(1.8) .80 \%$ yield.

Representative Polymerization of $\varepsilon$-tCL with MTBD. $\varepsilon$-tCL (100 mg, $0.768 \mathrm{mmol}$, [1M]) was dissolved in half of the total $\mathrm{CHCl}_{3}(0.77 \mathrm{~mL})$ used in the reaction and added to a solution of 1 octadecanethiol $(4.4 \mathrm{mg}, 0.015 \mathrm{mmol})$ and MTBD $(5.9 \mathrm{mg}, 0.039 \mathrm{mmol})$ made with the remaining $\mathrm{CHCl}_{3}$. Reaction was left to stir for $80 \mathrm{~min}$, quenched with benzoic acid $(3.0 \mathrm{mg})$, and solvent removed in vacuo to yield a white film. Conversion was determined by NMR and purified by precipitation from DCM with hexanes. ${ }^{1} \mathrm{H} N M R\left(\mathrm{CDCl}_{3}\right): \delta=3.53-3.49\left(\mathrm{t}, 2 \mathrm{H} ;\left(\mathrm{CH}_{2}\right){ }_{16} \mathrm{CH}_{2} \mathrm{~S}\right)$, 2.87-2.82 (t, 66H; PB CH $2 \mathrm{~S}), 2.55-2.50$ (t, $\left.\sim 58 \mathrm{H} ; \mathrm{PB} \mathrm{C}(\mathrm{O}) \mathrm{CH}_{2}\right), 1.71-1.52(\mathrm{~m}, \sim 128 \mathrm{H} ; \mathrm{PB}$ $\left.\mathrm{CH}_{2}\right), 1.43-1.33(\mathrm{~m}, \sim 61 \mathrm{H}$; PB CH 2$), 0.89-0.85$ (t, 3H; $\left.\mathrm{CH}_{3} \mathrm{CH}_{2}\right)$. GPC (UV-vis): $M_{\mathrm{n}}\left(M_{\mathrm{w}} / M_{\mathrm{n}}\right)=$ $8400 \mathrm{~g} \mathrm{~mol}^{-1}$ (1.62). $85 \%$ yield.

Binding Study Procedure. The titration method and the linear forms of the binding equations were used as previously described..$^{22}$ Briefly, two stock solutions were made for this experiment: solution $\mathrm{A}$ was $533.3 \mathrm{mM} \varepsilon$-tCL $(78.12 \mathrm{mg}, 0.6 \mathrm{mmol})$ dissolved in $\mathrm{C}_{6} \mathrm{D}_{6}(1.5 \mathrm{~mL}, 16.93 \mathrm{mmol})$. Solution B was $20 \mathrm{mM} 1$ (7.4 mg, $0.20 \mathrm{mmol})$ dissolved in $\mathrm{C}_{6} \mathrm{D}_{6}(1.0 \mathrm{~mL}, 11.29 \mathrm{mmol})$. Several NMR samples were made from the above solutions using a calibrated volumetric pipet and dried NMR tubes. The binding constant was determined by monitoring the chemical shift of the orthoaromatic protons of the thiourea and error was determined by linear regression at the $95 \%$ confidence interval. Plot of the data using the Lineweaver-Burke form of the binding equation is given in the Supporting Information Figure $\mathrm{S} 11 .{ }^{27-29}$

Determining Thermodynamics of tCL ROP. In a variable temperature NMR probe, a sample of $100 \mathrm{mg}(0.77 \mathrm{mmol})$ of $\varepsilon$-tCL was reacted with $0.015 \mathrm{mmol}$ initiator and $0.19 \mathrm{mmol}$ TBD and the concentration of monomer was determined at multiple temperatures from 293 to $333 \mathrm{~K}$. The concentrations were recorded twice, once upon heating and once upon cooling; the values at each temperature were within error of each other. These concentrations are the equilibrium monomer concentration $\left([\mathrm{M}]_{\mathrm{eq}}=1 / \mathrm{K}_{\mathrm{eq}}\right)^{17}$ at each temperature. The thermodynamic values were 
extracted from a van't Hoff plot of the data, see Supporting Information Figure S12, and error was determined by linear regression at the $95 \%$ confidence interval. 


\section{AKNOWLEDGEMENTS}

The authors declare no competing financial interest. This research was supported by NIH under RI-INBRE (8 P20 GM103430-12) and the University of Rhode Island. 


\section{LIST OF REFERENCES}

(1) Kamber, N. E.; Jeong, W.; Waymouth, R. M.; Pratt, R. C.; Lohmeijer, B. G. G.; Hedrick, J. L. Chem. Rev. 2007, 107, 5813-5840.

(2) Kiesewetter, M. K.; Shin, E. J.; Hedrick, J. L.; Waymouth, R. M. Macromolecules 2010, 43, 2093-2107.

(3) Zhang, Y.; Schmitt, M.; Falivene, L.; Caporaso, L.; Cavallo, L.; Chen, E. Y.X. J. Am. Chem. Soc. 2013, 135, 17925-17942.

(4) Scholten, M. D.; Hedrick, J. L.; Waymouth, R. M. Macromolecules 2008, 41, 7399-7404.

(5) Kricheldorf, H. R.; Schwarz, G. J. Macromol. Sci., Part A: Pure Appl.Chem. 2007, 44, 625-649.

(6) Dove, A. P. ACS Macro Lett. 2012, 1, 1409-1412.

(7) Bang, E.-K.; Gasparini, G.; Molinard, G.; Roux, A.; Sakai, N.; Matile, S. J. Am. Chem. Soc. 2013, 135, 2088-2091.

(8) Nishikubo, T.; Kameyama, A.; Kawakami, S. Macromolecules 1998, 31, 4746-4752.

(9) Overberger, C. G.; Weise, J. K. J. Am. Chem. Soc. 1968, 90, 3533-3537.

(10) Overberger, C. G.; Weise, J. K. J. Am. Chem. Soc. 1968, 90, 3538-3543.

(11) Seefried, C. G.; Kodeske, J. V. Polym. Eng. Sci. 1976, 16, 526-528.

(12) Fritze, P. E. (Union Carbide: USA). Thiolactone Polymerization and Catalysts. US Patent 3,755,268, June 16, 1972.

(13) Kricheldorf, H. R.; Lee, S.; Schittenhelm, N. Macromol. Chem. Phys. 1998, 199, 273-282.

(14) Shimokawa, K.; Kato, M.; Matsumura, S. Macromol. Chem. Phys. 2011, 212, 150-158.

(15) Kato, M.; Toshima, K.; Matsumura, S. Biomacromolecules 2005, 6, 2275-2280.

(16) Lohmeijer, B. G. G.; Pratt, R. C.; Leibfarth, F.; Logan, J. W.; Long, D. A.; Dove, A. P.; Nederberg, F.; Choi, J.; Wade, C.; Waymouth, R. M.; Hedrick, J. L. Macromolecules 2006, 39, 8574-8583. 
(17) Duda, A.; Kowalski, A. In Handbook of Ring-Opening Polymerization; Dubois, P., Coulembier, O., Raquez, J.-M., Eds.; Wiley-VCH: Weinheim, Germany, 2009; pp 1-52.

(18) Kaljurand, I.; Kütt, A.; Sooväli, L.; Rodima, T.; Mäemets, V.; Leito, I.; Koppel, I. A. J. Org. Chem. 2005, 70, 1019-1028.

(19) Schwesinger, R.; Schlemper, H.; Hasenfratz, C.; Willaredt, J.; Dambacher, T.; Breuer, T.; Ottaway, C.; Fletschinger, M.; Boele, J.; Fritz, H.; Putzas, D.; Rotter, H. W.; Bordwell, F. G.; Satish, A. V.; Ji, G.; Peters, E.; Peters, K.; Schnering, H. G.; Von Walz, L. Liebigs Ann. 1996, 1996, 1055-1081.

(20) Augustin-Nowacka, D.; Chmurzynski, L. Anal. Chim. Acta 1999, 381, 215-220.

(21) Brown, H. A.; De Crisci, A. G.; Hedrick, J. L.; Waymouth, R. M. ACS Macro Lett. 2012, $1,1113-1115$.

(22) Kazakov, O. I.; Datta, P. P.; Isajani, M.; Kiesewetter, E. T.; Kiesewetter, M. K. Macromolecules 2014, 47, 7463-7468.

(23) Colebrook, L. D.; Tarbell, D. S. Proc. Natl. Acad. Sci. U. S. A. 1961, 47, 993-996.

(24) Coady, D. J.; Engler, A. C.; Horn, H. W.; Bajjuri, K. M.; Fukushima, K.; Jones, G. O.; Nelson, A.; Rice, J. E.; Hedrick, J. L. ACS Macro Lett. 2012, 1, 19-21.

(25) Pratt, R. C.; Lohmeijer, B. G. G.; Long, D. A.; Lundberg, P. N. P.; Dove, A. P.; Li, H.; Wade, C. G.; Waymouth, R. M.; Hedrick, J. L. Macromolecules 2006, 39, 7863-7871.

(26) Shimokawa, K.; Kato, M.; Matsumura, S. Macromol. Chem. Phys. 2011, 212, 150-158.

(27) Deranleau, D. A. J. Am. Chem. Soc. 1969, 91, 4044-4049.

(28) Horman, I.; Dreux, B. Anal. Chem. 1983, 55, 1219-1221.

(29) Peters, S. J.; Stevenson, C. D. J. Chem. Educ. 2004, 81, 715-720. 


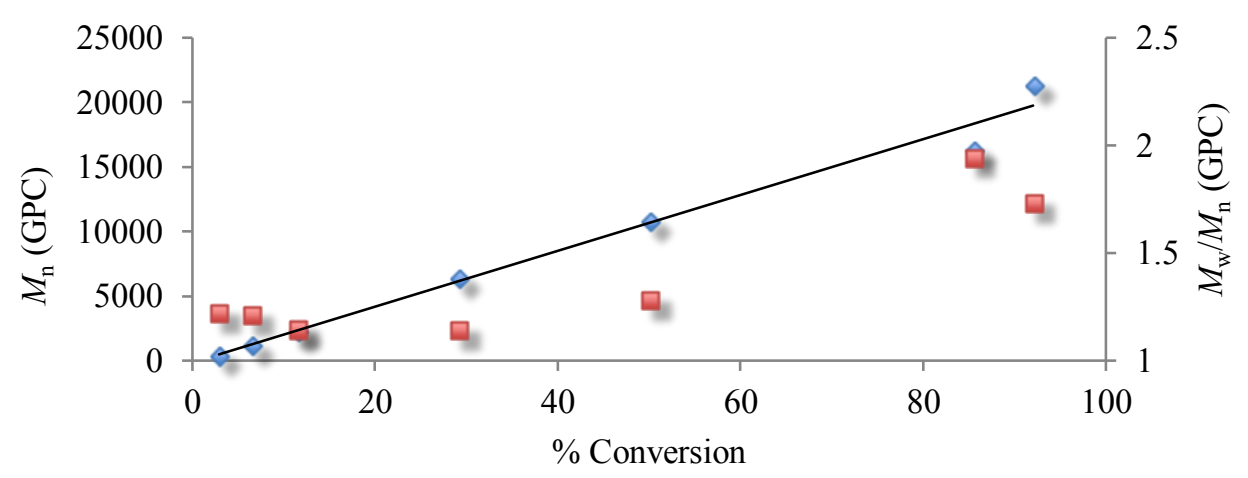

Figure S1. $M_{\mathrm{n}}$ (blue) and $M_{\mathrm{w}} / M_{\mathrm{n}}$ (red) vs. \% Conversion for the MTBD catalyzed ROP of $\varepsilon$-tCL; $[\mathrm{M}]_{0} /[]_{0}=100$.

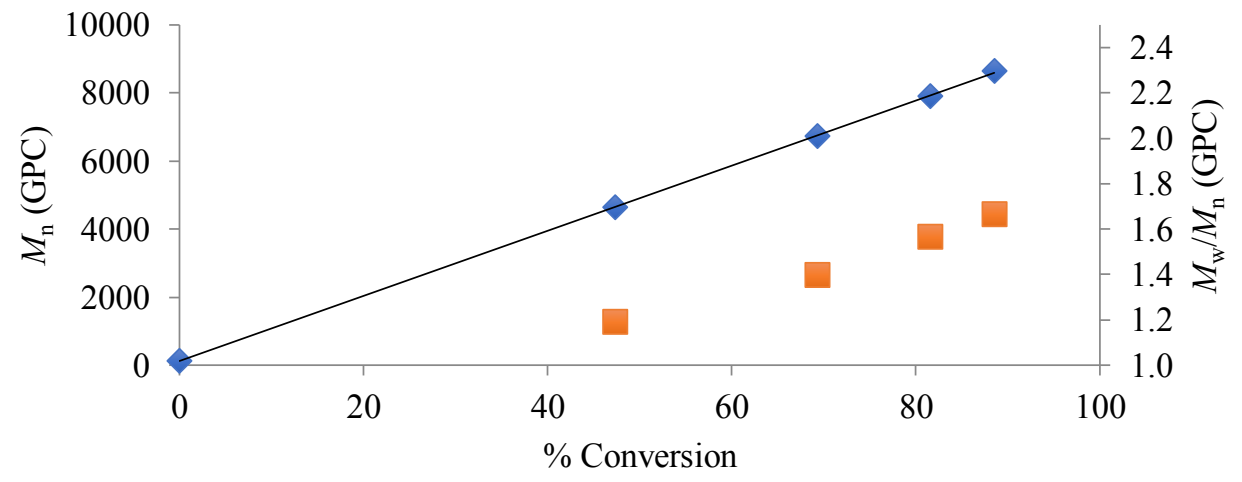

Figure S2. $M_{n}$ (blue) and $M_{w} / M_{n}$ (orange) vs. \% Conversion for the DBU catalyzed ROP of $\varepsilon$ $\mathrm{tCL}$. 

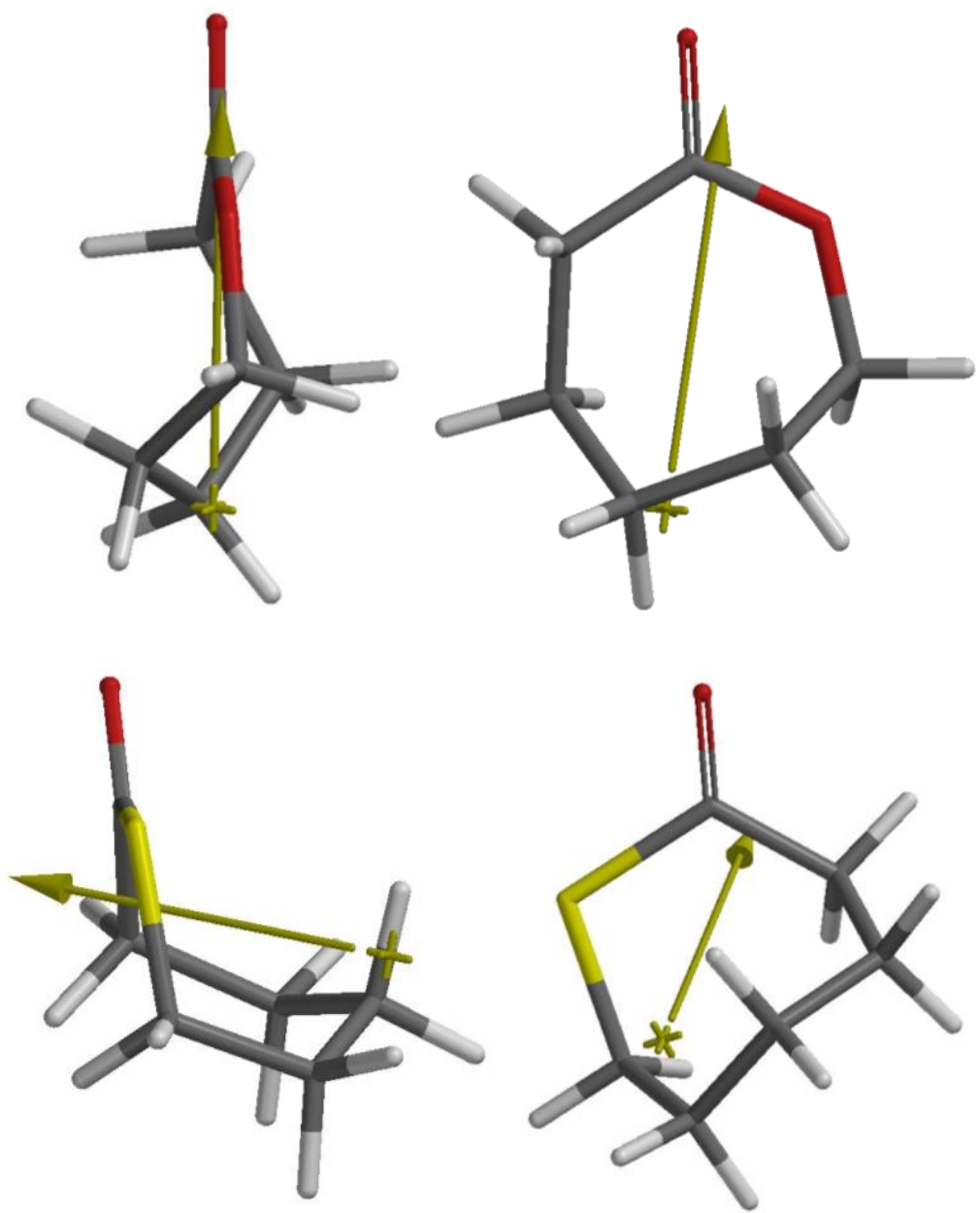

Figure S3. Two views of a calculated equilibrium geometry (Spartan '14, DFT B3LYP 6-31G*, gas phase) with calculated dipole moment vector of (upper) CL and, (lower) $\varepsilon$-tCL. 


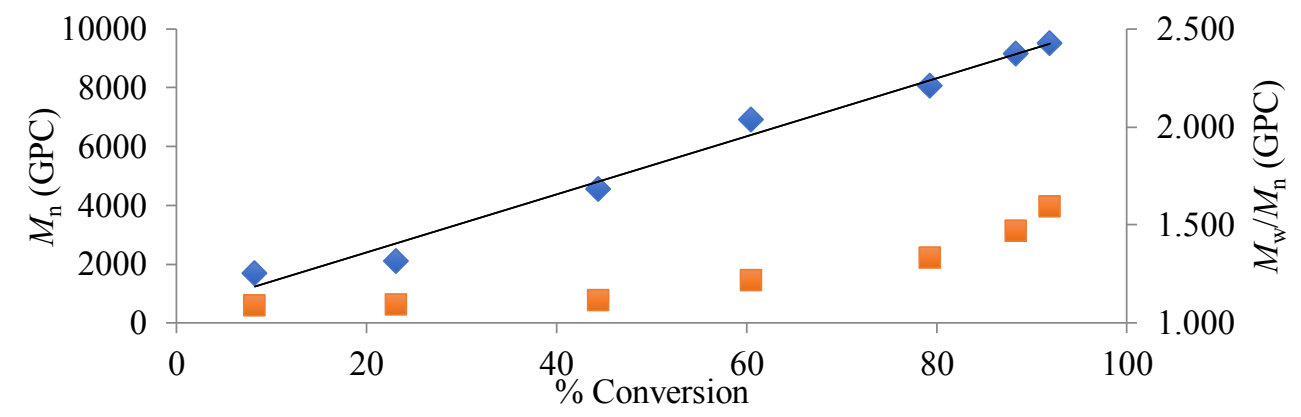

Figure S4. $M_{\mathrm{n}}$ (blue) and $M_{\mathrm{w}} / M_{\mathrm{n}}$ (orange) vs. \% Conversion for the DBU/1 catalyzed ROP of $\varepsilon$ tCL.
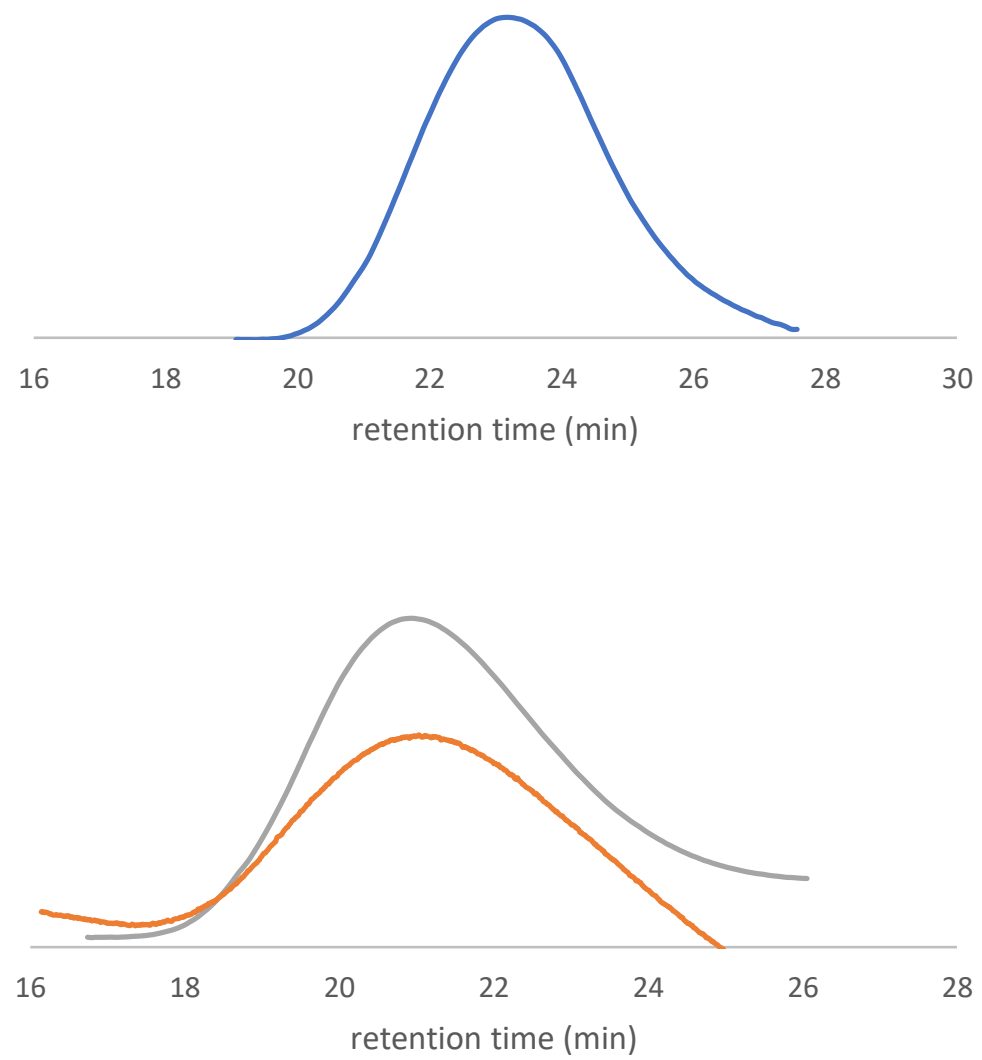

Figure S5. GPC Trace of MTBD/1 initiated by octadecylthiol; RI detector (above) and MTBD/1 initiated by 1-pyrenebutanol; RI in grey and UV (300 nm) orange (below). 


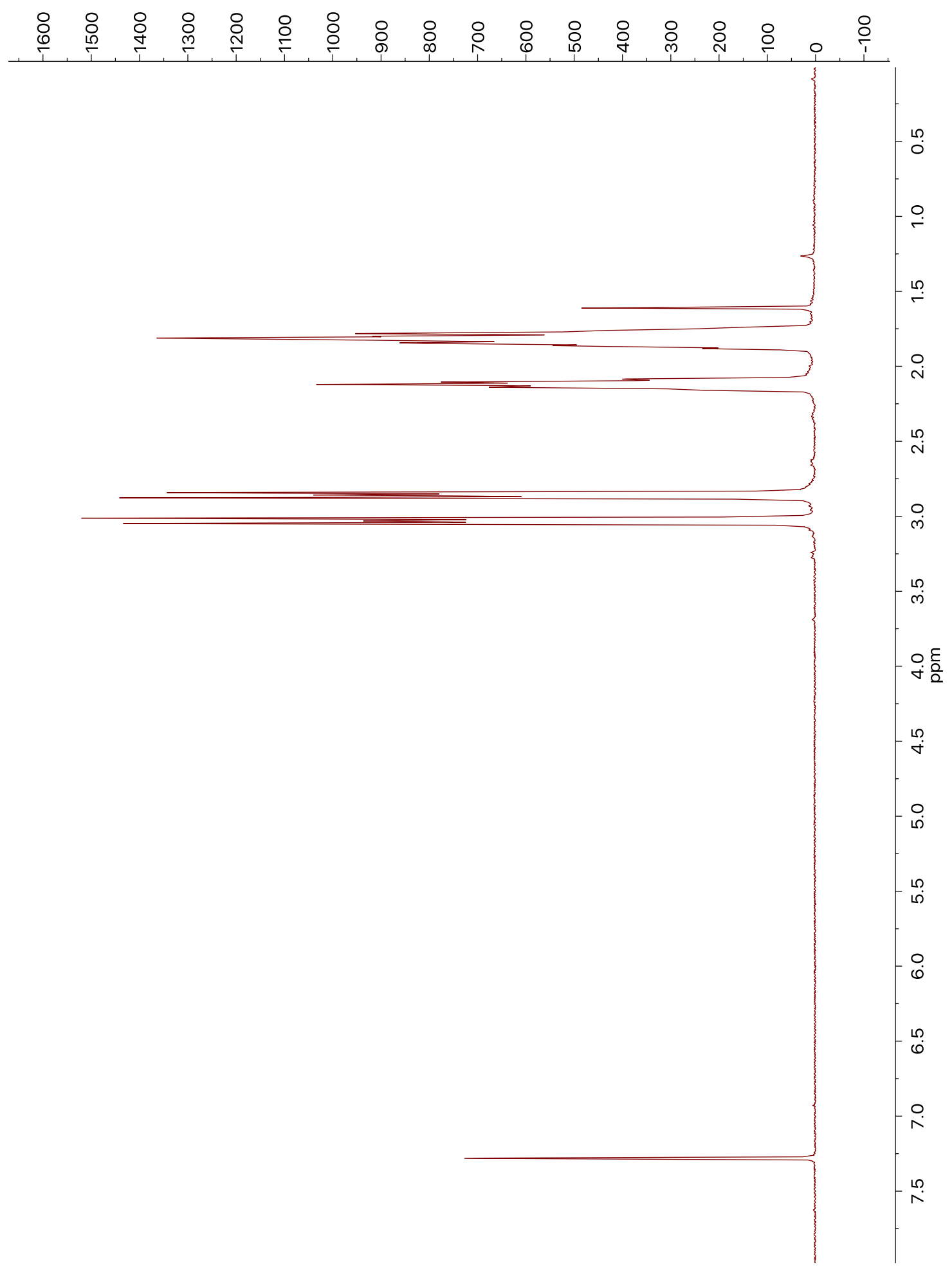

Figure S6. ${ }^{1} \mathrm{H}$ NMR $\left(\mathrm{CDCl}_{3}, 300 \mathrm{MHz}\right)$ of $\varepsilon$-tCL. 


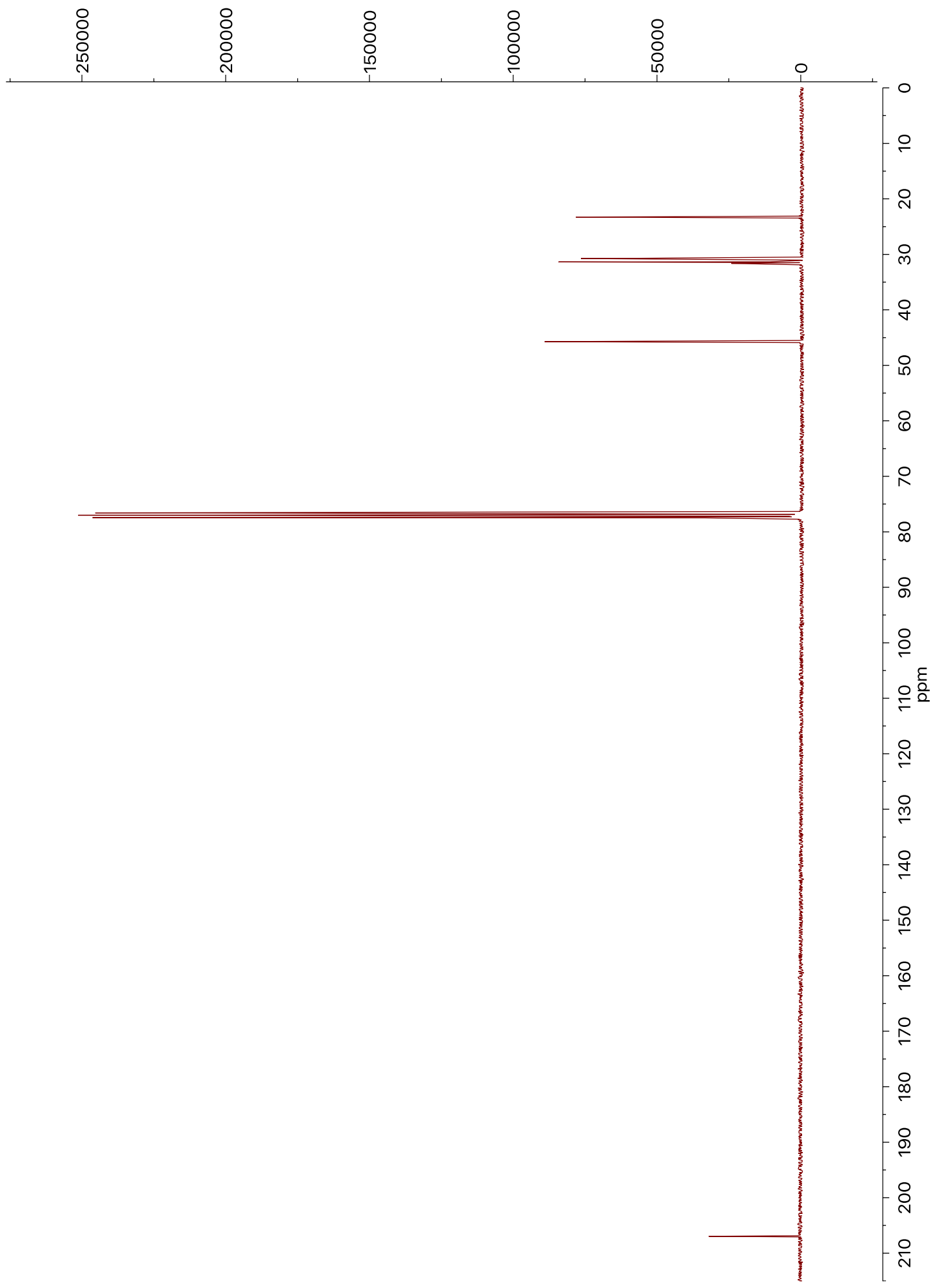

Figure S7. ${ }^{13} \mathrm{C}$ NMR $\left(\mathrm{CDCl}_{3}, 75 \mathrm{MHz}\right)$ of $\varepsilon$-tCL. 


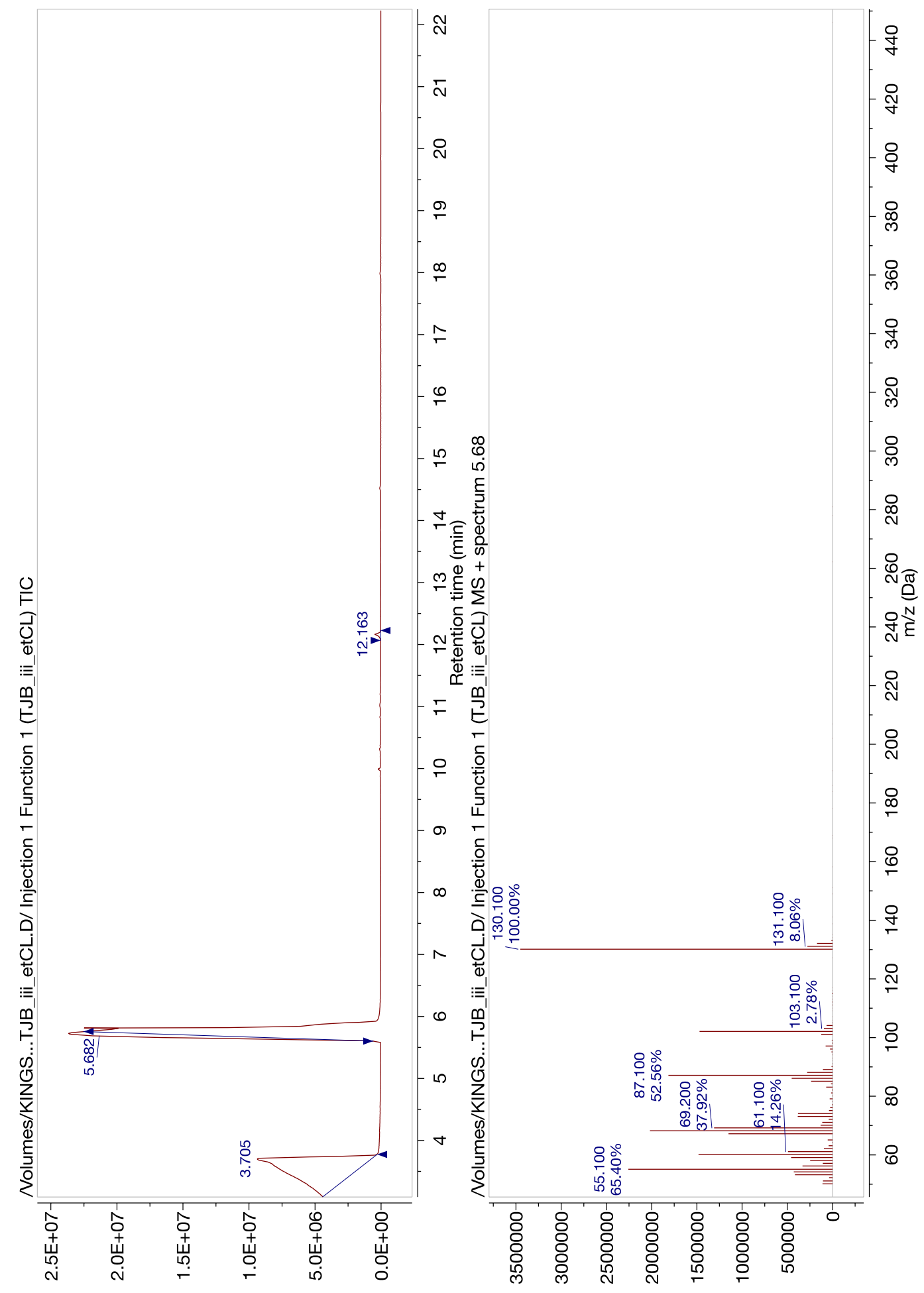

Figure S8. GC-MS (electron impact) of tCL. The GC sample was prepared in THF and the peak at 3.7 is due to solvent and 12.2 is BHT (THF stabilizer). 


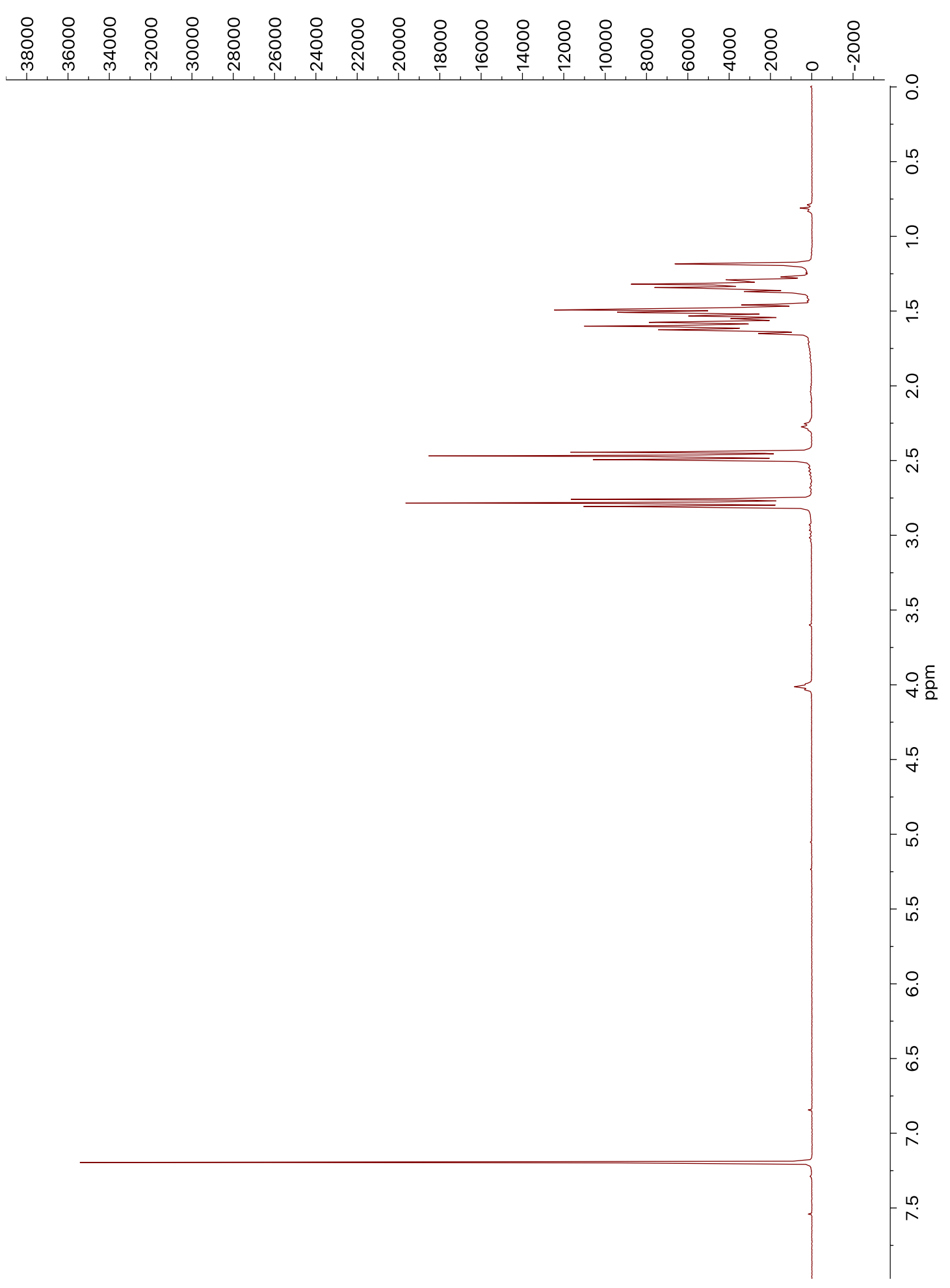

Figure S9. ${ }^{1} \mathrm{H} \mathrm{NMR}\left(\mathrm{CDCl}_{3}, 300 \mathrm{MHz}\right)$ of poly- $\varepsilon$-tCL. 


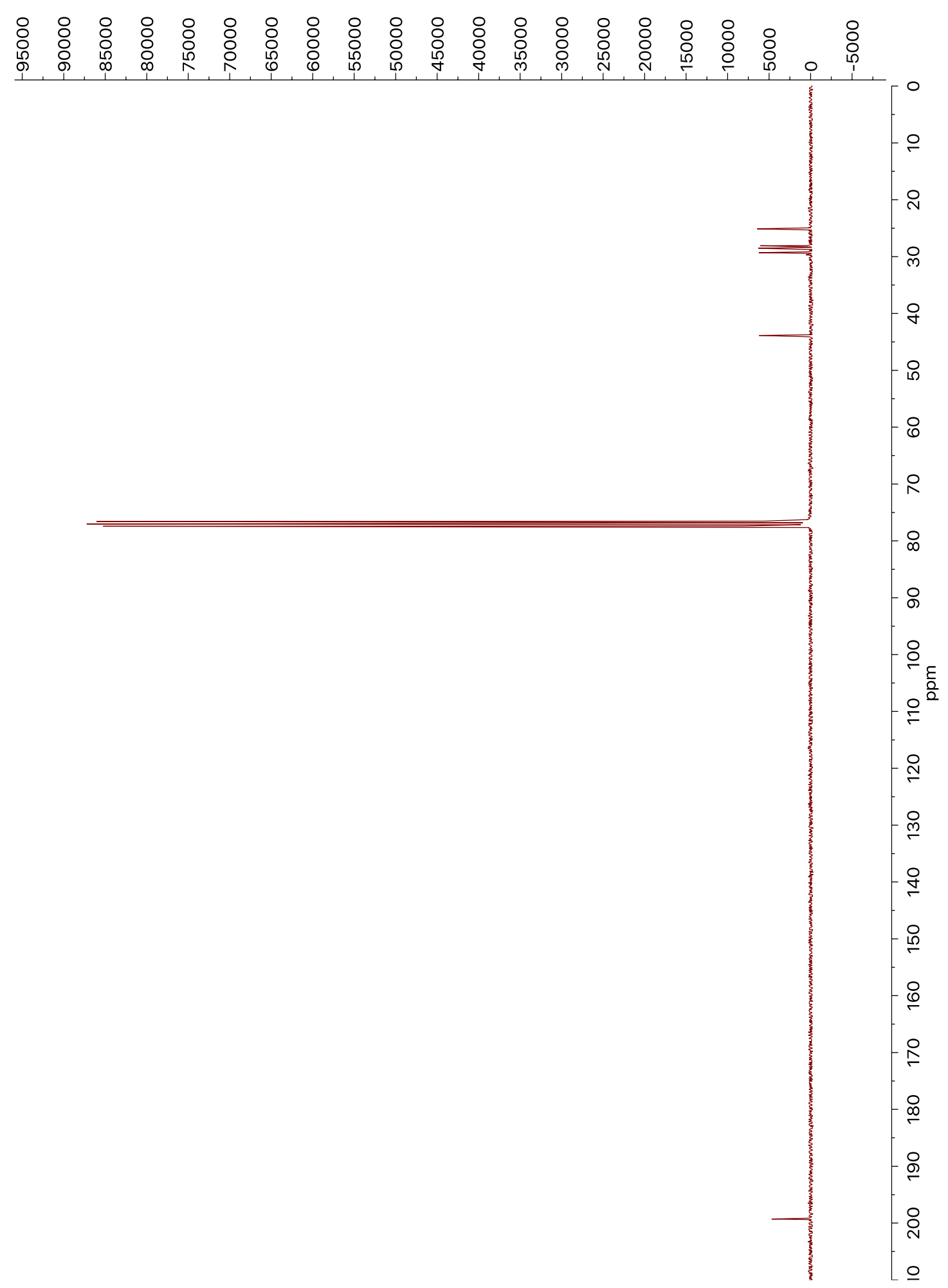

Figure $\mathrm{S} 10 .{ }^{13} \mathrm{C} \mathrm{NMR}\left(\mathrm{CDCl}_{3}, 75 \mathrm{MHz}\right)$ of poly- $\varepsilon$-tCL. 
$\Delta \delta /[$ base $]=-\mathrm{K}_{\text {eq }} \Delta \delta+\mathrm{K}_{\text {eq }} \delta \mathrm{c}$

Where:

$\Delta \delta$ is the difference between the chemical shift of the observed ortho-protons in the TU-Base mixture and of pure TU;

$\delta_{\mathrm{c}}$ is the chemical shift of the ortho-protons of TU in the complex, TU-Base;

$\mathrm{K}_{\mathrm{eq}}$ is the binding constant between $\mathbf{1}$ and a Base.

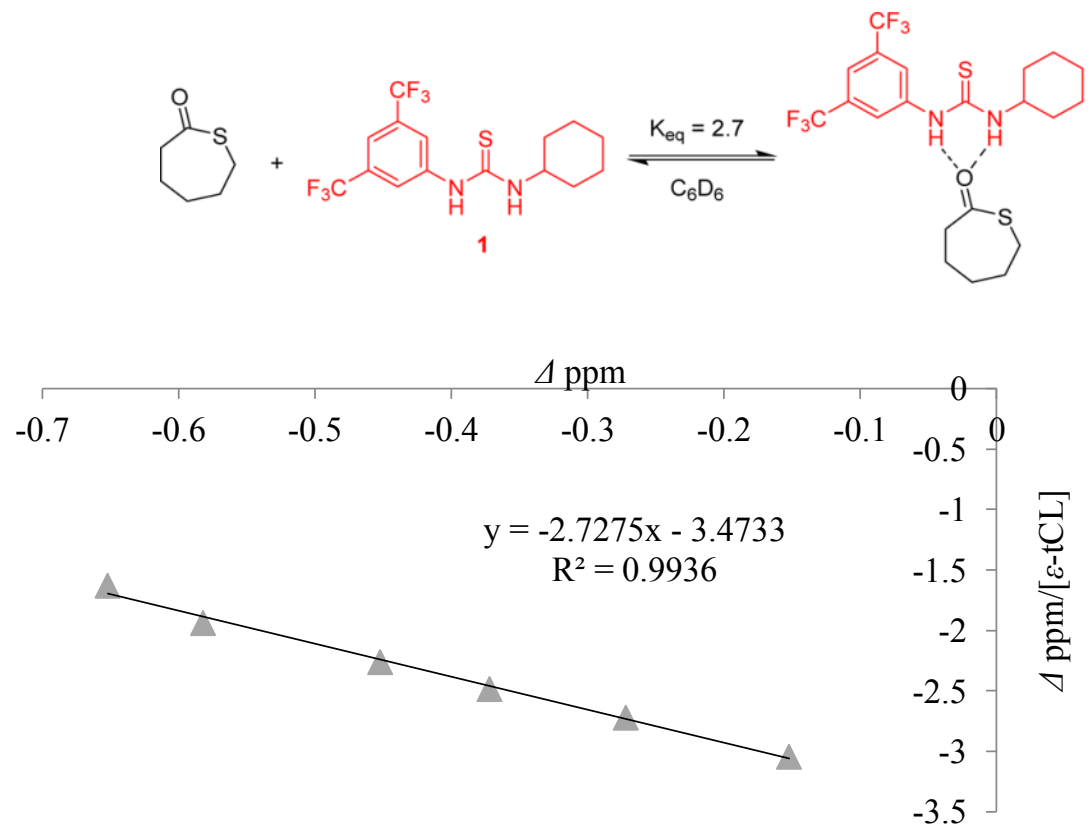

Figure $\mathrm{S} 11$. Equation $22,27-29$ and plot used to determine the binding constant, $\mathrm{K}_{\mathrm{eq}}$, between $\varepsilon^{-}$ tCL and 1. 


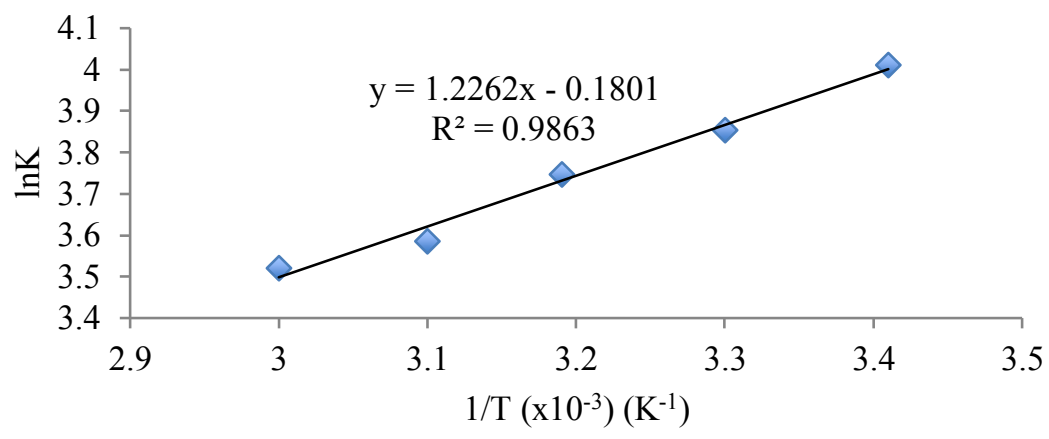

$$
\ln \frac{1}{[M]_{e q}}=\frac{-\Delta H_{p}^{\circ}}{R T}+\frac{\Delta S_{p}^{\circ}}{R}
$$

Figure S12. Van't Hoff plot for the TBD catalyzed ROP of $\varepsilon$-tCL.

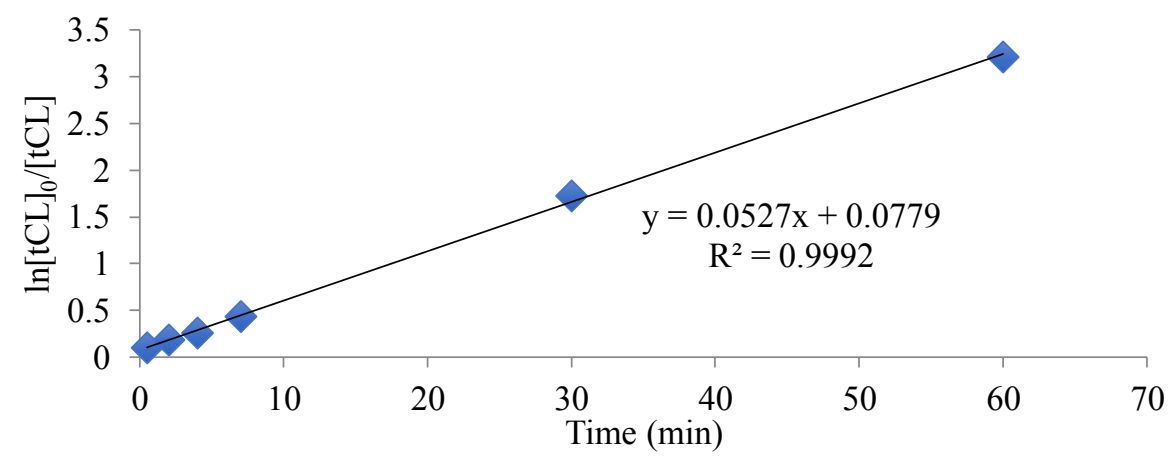

Figure S13. First order evolution of [tCL] with time. Reaction conditions: $100 \mathrm{mg}(0.77 \mathrm{mmol}$, [1M]) tCL in $\mathrm{CHCl}_{3} ; 0.015 \mathrm{mmol}$ octadecylthiol; $0.038 \mathrm{mmol}$ MTBD. 


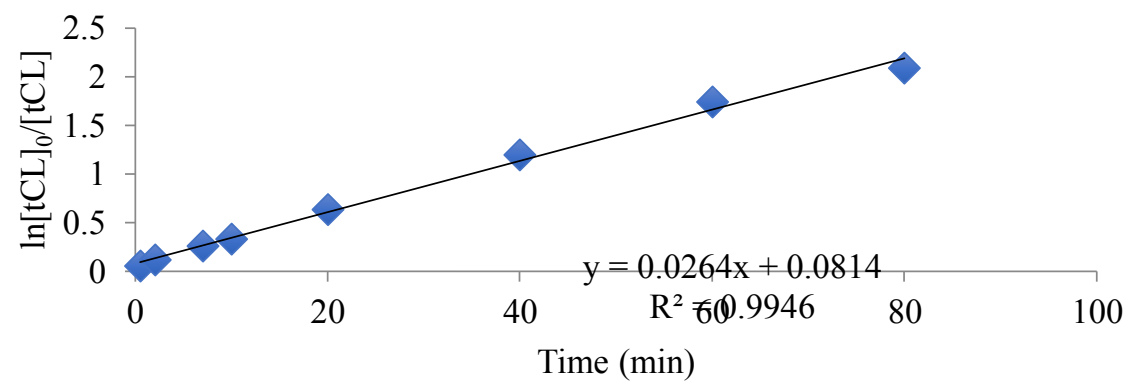

Figure S14. First order evolution of [tCL] with time. Reaction conditions: $100 \mathrm{mg}(0.77 \mathrm{mmol}$, [1M]) tCL in $\mathrm{CHCl}_{3} ; 0.015 \mathrm{mmol}$ octadecylthiol; $0.038 \mathrm{mmol}$ each MTBD and 1.

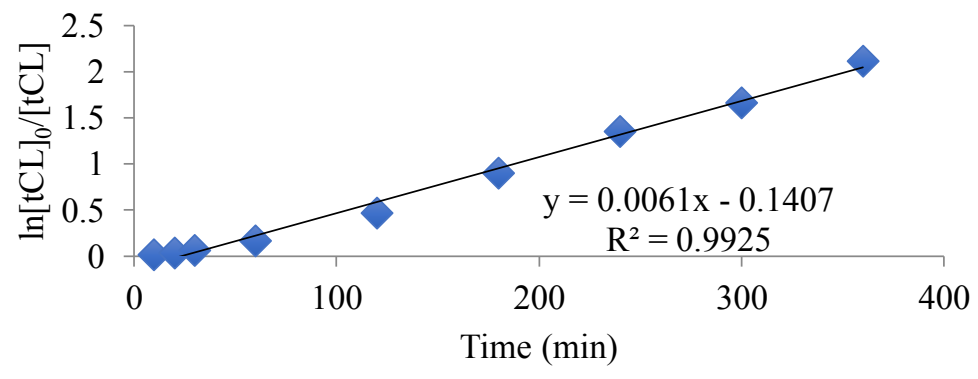

Figure S15. First order evolution of [tCL] with time. Reaction conditions: $100 \mathrm{mg}(0.77 \mathrm{mmol}$, [1M]) tCL in $\mathrm{CHCl}_{3} ; 0.015 \mathrm{mmol} 1$-pyrenebutanol; $0.038 \mathrm{mmol}$ each MTBD and 1. 


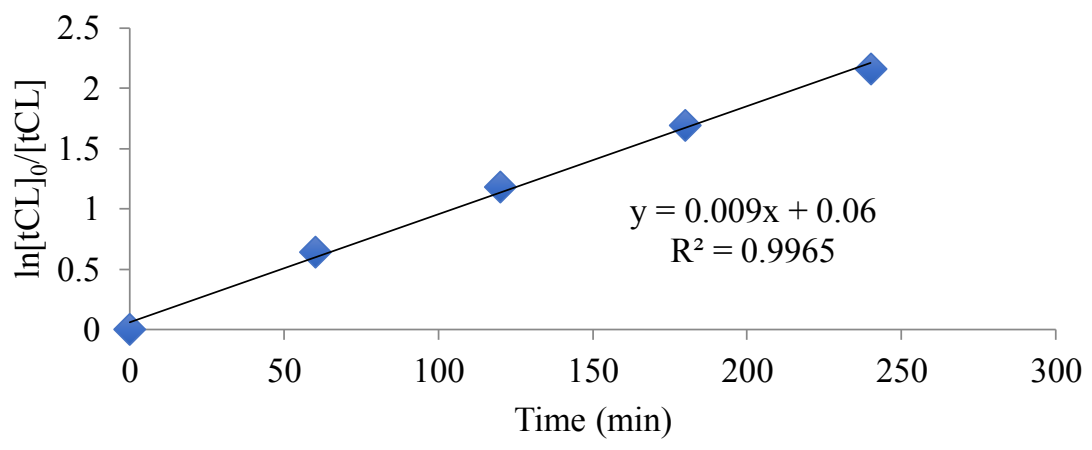

Figure S16. First order evolution of [tCL] with time. Reaction conditions: $100 \mathrm{mg}(0.77 \mathrm{mmol}$, [1M]) tCL in $\mathrm{CHCl}_{3} ; 0.015 \mathrm{mmol}$ octadecylthiol; $0.038 \mathrm{mmol} \mathrm{DBU}$.

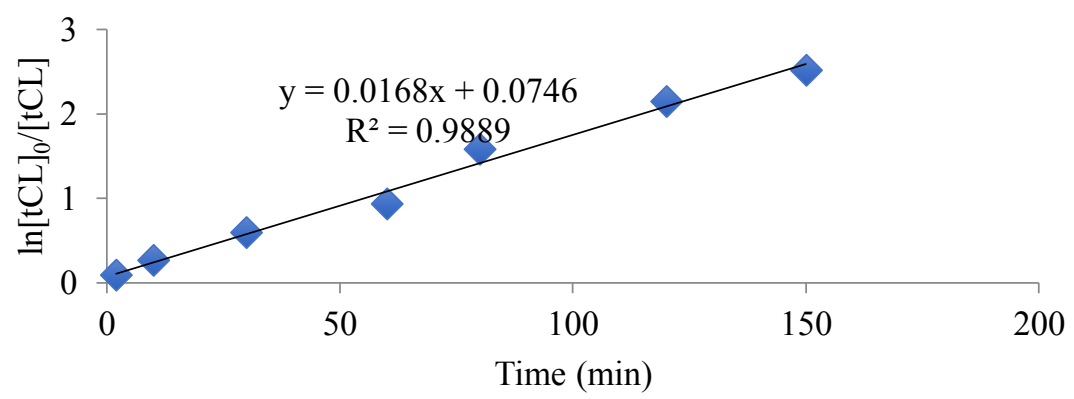

Figure S17. First order evolution of [tCL] with time. Reaction conditions: $100 \mathrm{mg}(0.77 \mathrm{mmol}$, [1M]) tCL in $\mathrm{CHCl}_{3} ; 0.015 \mathrm{mmol}$ octadecylthiol; $0.038 \mathrm{mmol}$ each DBU and 1. 


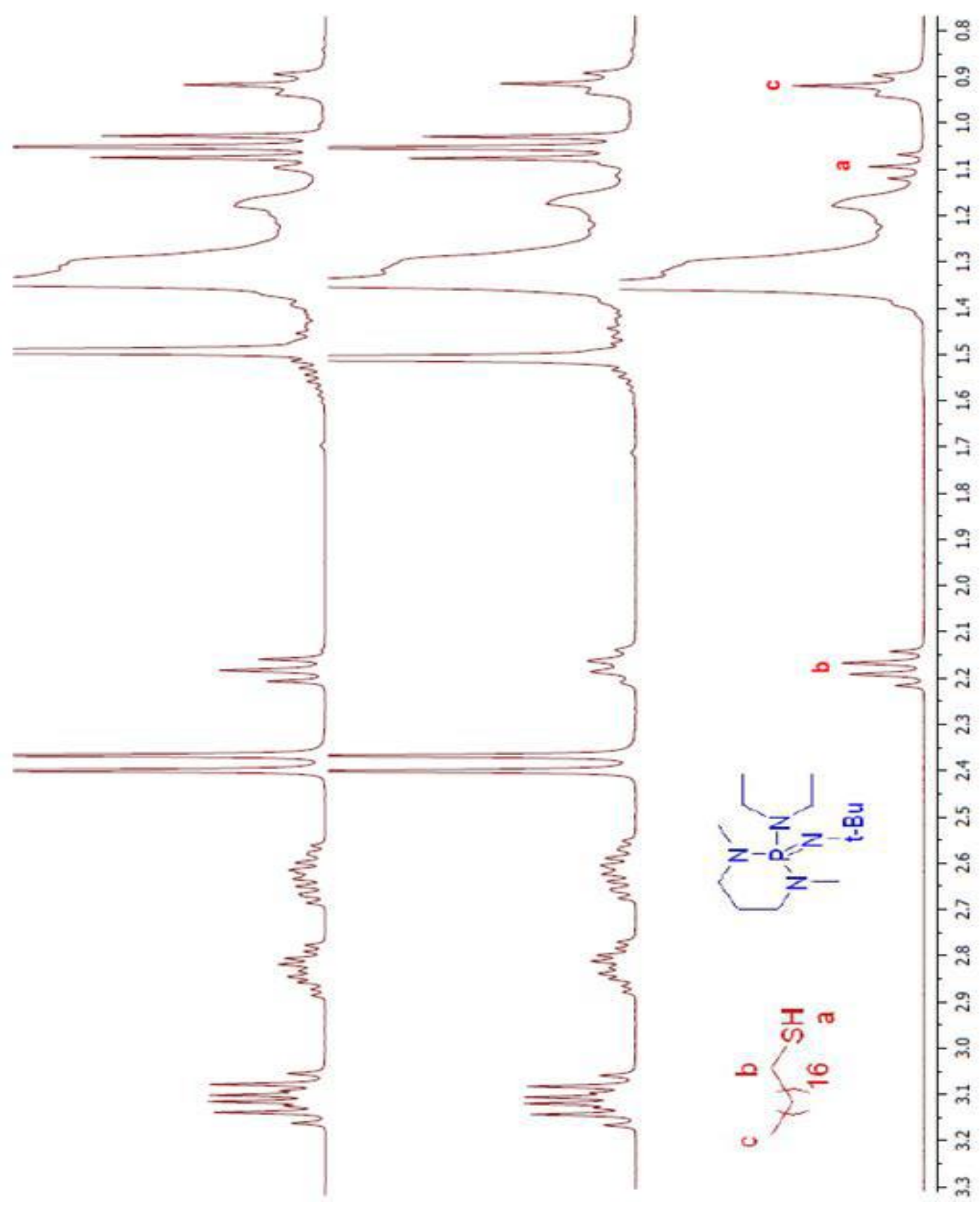

Figure S18. Upfield portions of ${ }^{1} \mathrm{H}$ NMR spectra $\left(\mathrm{C}_{6} \mathrm{D}_{6}, 300 \mathrm{MHz}\right)$ of (lower) $100 \mathrm{mM}$ octadecylthiol of thiol; (middle) $10 \mathrm{mM}$ octadecylthiol and $10 \mathrm{mM}$ BEMP; (upper) $100 \mathrm{mM}$ octadecylthiol and $100 \mathrm{mM} \mathrm{BEMP.} \mathrm{The} \mathrm{'a'} \mathrm{and} \mathrm{'} b$ ' protons become decoupled in the presence of BEMP due to rapid proton exchange. 


\section{CHAPTER 3}

Published in ACS Macro Letters, March 2017

Triclocarban: Commercial Antibacterial and Highly Effective H-Bond Donating Catalyst for Ring-Opening Polymerization

Nayanthara U. Dharmaratne, Jinal U. Pothupitiya, Timothy J. Bannin, Oleg I. Kazakov, and Matthew Kiesewetter.

Chemistry, University of Rhode Island, Kingston, RI USA

Corresponding Author: Matthew Kiesewetter, Ph.D.

Chemistry

University of Rhode Island

325C, Beaupre Hall, 140 Flagg Rd.

Kingston, RI, 02881, USA

Phone: $+1-401-874-2619$

Email address: $\underline{\text { mkiesewetter@chm.uri.edu }}$ 


\section{CHAPTER 3}

\section{ABSTRACT}

The antibacterial compound, triclocarban (TCC), is shown to be a highly effective $\mathrm{H}$-bond donating catalyst for ring-opening polymerization (ROP) when applied with an $\mathrm{H}$-bond accepting base cocatalyst. These ROPs exhibit the characteristics of "living" polymerizations. TCC is shown to possess the high activity characteristic of urea (vs thiourea) $\mathrm{H}$-bond donors. The urea class of $\mathrm{H}$-bond donors is shown to remain highly active in $\mathrm{H}$-bonding solvents, a trait that is not displayed by the corresponding thiourea $\mathrm{H}$-bond donors. Two $\mathrm{H}$-bond donating ureas that are electronically similar to TCC are evaluated for their efficacy in ROP, and a mechanism of action is proposed. This "off -the-shelf" $\mathrm{H}$-bond donor is among the most active and most controlled organocatalysts for the ROP of lactones. 


\section{RESULTS AND DISCUSSION}

$\mathrm{H}$-bond mediated ring-opening polymerization (ROP) has attracted interest due to the highly controlled nature of these transformations. ${ }^{1-4}$ These mild, highly functional group tolerant catalysts, especially the bimolecular systems consisting of a (thio)urea $\mathrm{H}$-bond donor plus $\mathrm{H}$ bond accepting base, have facilitated the construction of precise polymer architectures, multiblocks, and well-defined systems. ${ }^{3,5-8}$ Targeted efforts by several groups toward rateaccelerated, H-bond mediated ROP seek to address a critical shortcoming of the field: low activity. ${ }^{9-12}$ For example, our group has recently disclosed the utility of urea $\mathrm{H}$-bond donors for rate accelerated $\mathrm{ROP} ;{ }^{13}$ thiourea $\mathrm{H}$-bond donors have been used in organocatalytic ROP for more than a decade, but are less active. ${ }^{3}$ Another barrier to the wide implementation of this chemistry is the paucity of commercially available $\mathrm{H}$-bond donors. Most (thio)urea catalysts are synthesized via a "click" reaction of an appropriate amine and iso(thio)cyanate. ${ }^{3,14,15}$ While simple, this stands in contrast to the wide array of readily available $\mathrm{H}$-bond accepting base cocatalysts and adds a synthetic step prior to conducting polymerization chemistry. Certainly, the ready availability of chemical reagents and catalysts facilitates the wide implementation of chemical transformations. In this context, the antibacterial compound, triclocarban (TCC, Figure 1), recently banned as a hand soap additive by the FDA, captured our attention. ${ }^{16}$ It is an electron-deficient biaryl urea, similar to the slate of urea and multiurea $\mathrm{H}$-bond donating catalysts that we recently showed to be highly

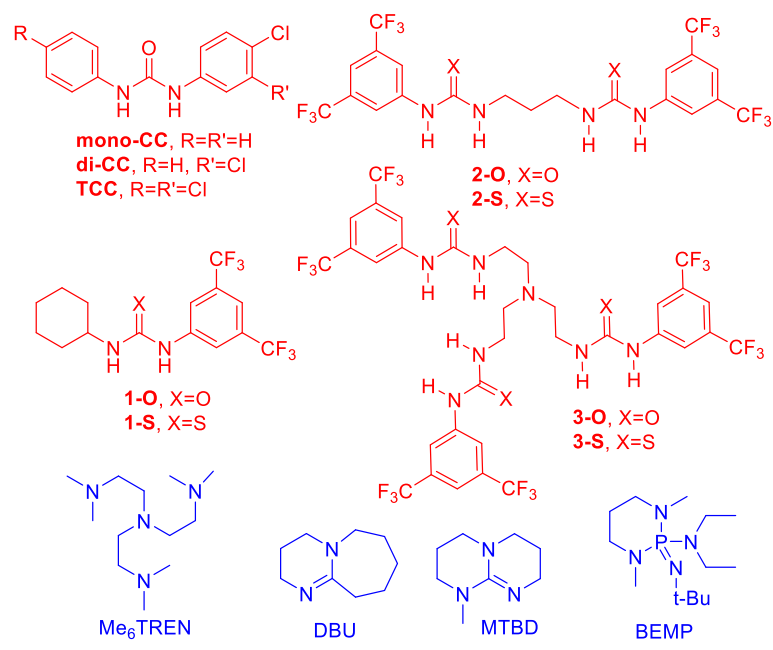


Figure 1. Base and (thio)urea cocatalysts evaluated for ROP.

active for ROP. ${ }^{13}$ While TCC has attracted considerable scientific interest as an antibacterial compound, possible bioaccumulate, and possible environmental toxin, we believe that this readily available compound has not previously been employed as a catalyst. ${ }^{17-19}$

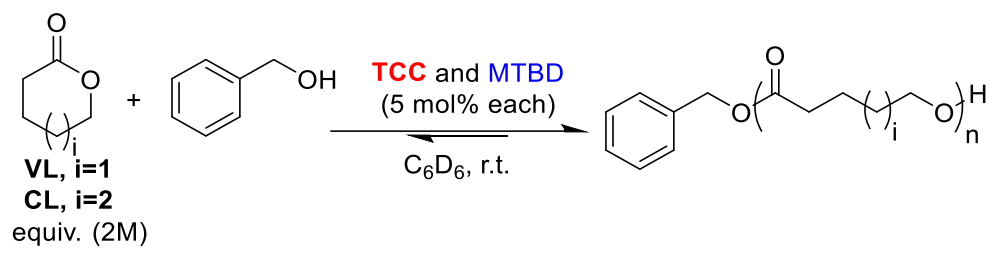

\begin{tabular}{|c|c|c|c|c|c|c|c|}
\hline \multirow{2}{*}{$\begin{array}{l}\text { Table } \\
1 .\end{array}$} & entry & mon. & {$[\mathrm{M}] \mathrm{o} /[\mathrm{I}] \mathrm{o}$} & time (min) & conv. $^{a}(\%)$ & $M_{\mathrm{n}^{\mathrm{b}}}(\mathrm{g} / \mathrm{mol})$ & $M_{w} / M^{b}$ \\
\hline & $1^{c}$ & VL & 100 & 81 & 90 & 18900 & 1.06 \\
\hline & 2 & & 100 & 22 & 91 & 19900 & 1.05 \\
\hline & 3 & & 50 & 14 & 90 & 8500 & 1.08 \\
\hline & 4 & & 200 & 46 & 90 & 35900 & 1.09 \\
\hline & 5 & & 500 & 125 & 90 & 72900 & 1.02 \\
\hline & 6 & $\mathrm{CL}$ & 100 & 132 & 90 & 21200 & 1.06 \\
\hline
\end{tabular}

MTBD and TCC Catalyzed ROP of VL and CL. Reaction conditions: VL or CL (1.0 mmol, 1 equiv, 2M), benzyl alcohol, $\mathrm{C}_{6} \mathrm{D}_{6}$. a) monomer conversion was monitored via ${ }^{1} \mathrm{H}$ NMR. b) $M \mathrm{n}$ and $M_{\mathrm{w}} / \mathrm{Mn}_{\mathrm{n}}$ were determined by GPC $\left(\mathrm{CH}_{2} \mathrm{Cl}_{2}\right)$ vs polystyrene standards. c) DBU (5 mol\%, $0.05 \mathrm{mmol}$ ) cocatalyst was employed (no MTBD).

The efficacy of TCC/amidine base combinations for the ROP of lactone monomers was evaluated, Table 1. All reactions were conducted in C6D6 and conversion monitored by $1 \mathrm{H}$ NMR. The guanidine base, MTBD, exhibited faster rates than the imine base, DBU, and it was used for further experimentation. The ROP of $\delta$-valerolactone $(V L)$ from benzyl alcohol is highly controlled, exhibiting the characteristics of a living polymerization: linear evolution of $\mathrm{Mn}$ vs conversion, first order consumption of monomer and Mn predictable from $[\mathrm{M}] 0 /[\mathrm{l}] 0$, (see Supporting Information Figures S1 and S2). This behavior is typical among organocatalysts for ROP.1,3 Initiation of a VL (1.0 mmol) ROP catalyzed by TCC/MTBD (0.05 mmol each) from 1 - 
pyrenebutanol $(0.02 \mathrm{mmol})$ and subsequent addition of a second monomer portion $(1.0 \mathrm{mmol})$ exhibits overlapping UV and refractive index traces in the gel permeation chromatogram (GPC) of the resulting polymer (see Supporting Information Figure S3), suggesting end group fidelity and a chain end that is susceptible to chain-extension. The TCC/MTBD (5 mol \%) cocatalysts are also effective for the ROP of $\varepsilon$-caprolactone $(\mathrm{CL})$, producing a similarly well-behaved ROP. The ROP rates exhibited by TCC/MTBD represent a significant advance over those exhibited by $1-S / M T B D$, yet the reactions remain highly controlled. By comparison, for $[M]_{0} /[]_{0}=50$ from benzyl alcohol in $\mathrm{C}_{6} \mathrm{D}_{6}$, the 1-S/MTBD-catalyzed ROPs of VL and $C L$ achieve full conversion in $110 \mathrm{~min}$ and $45 \mathrm{~h}$, respectively (c.f. Table 1). ${ }^{13}$ Entry 2 (Table 1 ) was attempted on a $200 \mathrm{mg}$ scale, producing nearly identical polyvalerolactone $\left(24 \min , 90 \%\right.$ conv, $M_{n}=18100, M_{w} / M_{n}=$ 1.04), which suggests that scale-up is feasible.

We have embarked on a research program aimed at mitigating the low activity of $\mathrm{H}$-bond mediated transformations without sacrificing the precise control typical of these catalysts. In this vein, electron deficient aryl ureas have proved to be particularly efficacious; our lab previously disclosed the rapid rates exhibited by mono-, bis-, and tris-urea $\mathrm{H}$-bond donors for the ROP of lactones. ${ }^{13}$ In general, urea $\mathrm{H}$-bond donors are more active for ROP than their corresponding thioureas. This trend extends to the urea anions which, besides being remarkably active and controlled catalysts for ROP, are much more active than the corresponding thiourea anions. ${ }^{10,12}$ The uncharged $\mathrm{H}$-bond donor 3-O, in combination with MTBD $(0.017 \mathrm{mmol}$ each), effects the ROP of VL $(1.0 \mathrm{mmol})$ from benzyl alcohol $(0.02 \mathrm{mmol})$ in $\mathrm{C}_{6} \mathrm{D}_{6}$ in $3 \mathrm{~min} .{ }^{13}$ While the analogous reaction with TCC/MTBD $(0.05 \mathrm{mmol}$ each) achieves full conversion in a slower 14 min, the commercial availability of the TCC catalyst is expected to be a boon to the wider application of this and similar systems. Additionally, the TCC/MTBD cocatalysts exhibit high selectivity for monomer (vs polymer). When a fully converted PVL reaction solution remains unquenched, the $M_{\mathrm{n}}$ and $M_{\mathrm{w}} / M_{\mathrm{n}}$ are minimally altered over an hour: $20 \mathrm{~min}, M_{n}=22300, M_{w} / M_{n}=1.02 ; 60 \mathrm{~min}, M_{n}=23900, M_{w} / M_{n}=1.03$ (c.f. Table 1, entry 2), which may constitute an advantage versus other highly active systems for ROP. ${ }^{10,12-13}$ 
Urea $\mathrm{H}$-bond donors remain active in polar, $\mathrm{H}$-bond accepting solvent. A long-standing limitation of $\mathrm{H}$-bond mediated catalysis is the often narrow window of nonpolar solvents in which these catalysts are operable..$^{20,21}$ We had previously observed that the urea $\mathrm{H}$-bond donor 3-O remains active in THF and hypothesized that TCC would exhibit similar behavior, and a solvent screen was conducted for the TCC/MTBD catalyzed ROP of VL (see Supporting Information Table S2). In DMF, the reaction time is extremely attenuated, and the reaction does not achieve $>83 \%$ conversion. In THF, the ROP remains highly active ( $90 \%$ conv in $30 \mathrm{~min}$ ), but $M_{\mathrm{w}} / \mathrm{Mn}_{\mathrm{n}}$ $(=1.23)$ broadens. The result in acetone is surprising in that the reaction rate does not slow versus $\mathrm{C}_{6} \mathrm{D}_{6}$, and the $M_{w} / M_{n}$ remains narrow, Table 2. The ROP rates for all thiourea $\mathrm{H}$-bond donors drop considerably versus their rates in $\mathrm{C}_{6} \mathrm{D}_{6},{ }^{13}$ Table 2 . The TCC/MTBD catalyzed ROP of $\mathrm{VL}$ in acetone- $d_{6}$ remains controlled and exhibits the characteristics of a "living" polymerization (see Supporting Information Figures S6 and S7). The polymer samples resulting from the initiation of a VL (1.0 mmol, $2 \mathrm{M})$ ROP from 1-pyrenebutanol $(0.02 \mathrm{mmol})$ catalyzed by TCC/MTBD (0.05 mmol each) and subsequent chain extension show overlapping UV and RI traces in the GPC (see Supporting Information Figure S8), which suggests end-group fidelity and that there is no initiation from the enol form of acetone- $d_{6}$.

\begin{tabular}{|c|c|c|c|c|c|}
\hline entry & solvent & time (min) & conv. $(\%)^{a}$ & $M_{n}^{b}$ & $M_{\mathrm{w}} / M_{\mathrm{n}}^{\mathrm{b}}$ \\
\hline 1 & benzene- $d_{6}$ & 22 & 91 & 19,900 & 1.06 \\
\hline 2 & acetone- $d_{6}$ & 22 & 89 & 19,400 & 1.11 \\
\hline 3 & chloroform- $d$ & 273 & 89 & 19,100 & 1.08 \\
\hline 4 & THF & 30 & 89 & 14,700 & 1.23 \\
\hline 5 & DMF & 600 & 83 & 9,000 & 1.41 \\
\hline
\end{tabular}

Table 2. Solvent Screen of TCC/MTBD cocatalyzed ROP of VL. a. Conversion determined by ${ }^{1} \mathrm{H}$ NMR. b. $M_{\mathrm{n}}$ and $M_{\mathrm{w}}$ were obtained by GPC. 
When TCC and Me6TREN cocatalysts (5 mol \% each) are applied for the ROP of L-lactide (1.0 $\mathrm{mmol}$ ) from benzyl alcohol $(0.01 \mathrm{mmol})$ in acetone- $d_{6}$, the ROP reaction exhibits "living" behavior (see Supporting Information Figures S11 and S12). In contrast to the ROP of VL, CL, or carbonate monomers, mild base cocatalysts are required for the ROP of lactide. ${ }^{21-24}$ The poly(lactide) was isolated and analyzed by selectively decoupled ${ }^{1} \mathrm{H} N M R$, revealing the polylactide (PLA) to be $\sim 90 \%$ isotactic (see Supporting Information Figure S16), which suggests minor epimerization. The MALDITOF analysis of the same PLA sample shows the presence of $\pm 72 \mathrm{~m} / \mathrm{z}$ repeat units, indicating that postpolymerization transesterification is occurring to a minor extent. This latter observation is in contrast to 2-S $\mathrm{H}$-bond donating catalyst, which effects the ROP of LA in the virtual absence of postpolymerization transesterifiation. ${ }^{23}$ $\mathrm{H}$-bond donating biaryl ureas were synthesized and applied in catalytic ROP to determine the origin of the enhanced rates of TCC (vs 1-0). These catalysts, here dubbed monoclocarban (mono-CC) $)^{25,26}$ and diclocarban (di-CC) ${ }^{26}$ in Figure 1, were applied to the ROP of VL in $\mathrm{C}_{6} \mathrm{D}_{6}$; we believe these molecules have not previously been used as catalysts. The TCC/MTBD (5 mol \% each) cocatalyzed ROP of VL (1.0 mmol, $2 \mathrm{M})$ from benzyl alcohol $(0.01 \mathrm{mmol})$ in $\mathrm{C}_{6} \mathrm{D}_{6}$ reaches $91 \%$ conversion in 22 min (Table 1). The $\mathrm{H}$-bond donors di-CC or mono-CC plus MTBD ( $5 \mathrm{~mol} \%$ each) exhibit similar activity to TCC, but di-CC is the most active of the three H-bond donors (88\% conversion in $15 \mathrm{~min}$ for $\mathrm{di}-\mathrm{CC}$ and $37 \mathrm{~min}$ for mono-CC). The ROP of VL catalyzed by di-CC/MTBD exhibits the characteristics of a "living" polymerization (see Supporting Information Figure S17). The similar rates exhibited by TCC and di-CC toward ROP may suggest that the additional chlorine atom in TCC (vs di-CC) is not essential for catalysis or that the additional electron withdrawing effects from the "extra" chlorine atom in TCC versus diCC are inhibitory to catalysis. The latter possibility recalls similar effects that have been observed for extremely electron deficient thioureas, ${ }^{27,28}$ and these observations suggest that the augmented activity of the biaryl TCC (vs 1-0) can be approximated by functionalization at a single aryl ring. Certainly, the increased efficacy of TCC (vs 1-0) for ROP calls into question the primacy of the bis(trifluoromethyl)aryl group, at least for urea $\mathrm{H}$-bond donors. ${ }^{27}$ While the commercial availability of TCC may be a boon to the application of $\mathrm{H}$-bond mediated 
transformations in polymer synthesis laboratories, we expect that the development of advanced catalysts architectures will benefit from the more synthetically modular catalyst scaffold of diCC.

The enhanced efficacy of TCC and all urea $\mathrm{H}$-bond donors in $\mathrm{C}_{6} \mathrm{D}_{6}$ could be attributed to the stronger binding of ureas vs thioureas to monomer. ${ }^{20}$ The limited solubility of TCC and $\mathbf{n}-\mathbf{O}$ in nonpolar solvent in the absence of base cocatalyst limits the extent to which we can quantitatively probe this hypothesis by measuring binding constants to monomer. For example, TCC is insoluble in benzene in the absence of $\mathrm{H}$-bond acceptor, and binding constants for this compound could not be measured. However, the binding constants of 1-0 and 1-S to CL were independently measured in $\mathrm{C}_{6} \mathrm{D}_{6}$ and are consistent with the long-held hypothesis: for $1-0, \mathrm{~K}_{\text {eq }}$ $=41 \pm 1(300 \mathrm{~K})$ and for $1-\mathrm{S}, \mathrm{K}_{\mathrm{eq}}=28 \pm 1(300 \mathrm{~K}) \cdot{ }^{24}$ However, a binding constant rationale cannot be used to explain the ROP activity observed in acetone. As expected, when the 1$\mathbf{O} /$ monomer binding study is repeated in acetone- $d_{6}$, there is no observed change in chemical shift of 1-0 up to $\sim 1000$ equiv of monomer, which suggests very weak ( $K_{\text {eq }} \sim 1$ ) or no binding in acetone- $d 6$. While we have previously observed $1-\mathbf{S}$ to exhibit a marked effect on a ROP reaction in the near absence of binding to monomer, ${ }^{29,30}$ these questions collectively reinforce a recently proposed mechanism. ${ }^{12}$

While this study was ongoing, "hyperactive" urea anions for ROP, generated by the action of alkoxides upon aryl and alkyl ureas, were disclosed; these systems are incredibly active yet controlled, exhibiting rates that rival traditional metal-based systems. ${ }^{12}$ The proposed mechanism of action whereby an active urea anion catalyst is generated by the deprotonation of a urea by alkoxide is distinct from traditional $\mathrm{H}$-bond mediated ROP by neutral catalysts, and we sought to investigate the feasibility of this mechanism for TCC/imine bases. As opposed to the quantitative deprotonation of TCC by potassium methoxide, one could envisage an equilibrium established between urea plus base and the corresponding salt, eq $1 .{ }^{1} \mathrm{H}$ NMR spectra in acetone- $d_{6}$ of TCC and TCC 


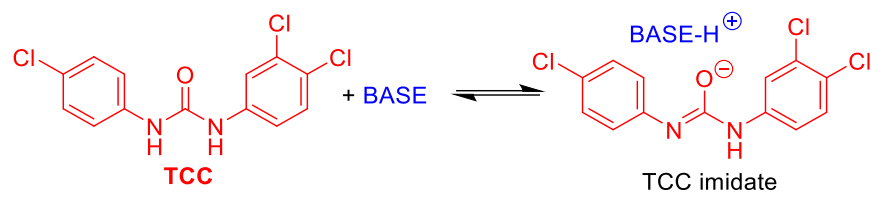

Equation 1. TCC-imidate equilibrium.

plus MTBD or DBU (5 mM each species) show an upfield shift of the TCC resonances upon treatment with base that would be associated with the formation of an anionic character at the urea (see Supporting Information Figure S20). Repeating this experiment with highly basic BEMP (Figure 1, BEMP- $\left.\mathrm{H}^{+} \mathrm{pK}_{\mathrm{a}}{ }^{\mathrm{MeCN}}=27.6\right)^{31}$ establishes a pattern of increased upfield shift with increasing $\mathrm{pK}_{\mathrm{a}}\left(\mathrm{MTBD}-\mathrm{H}^{+} \mathrm{pK}_{\mathrm{a}}{ }^{\mathrm{MeCN}}=25.4 ; \mathrm{DBU}-\mathrm{H}^{+} \mathrm{pK}_{\mathrm{a}}{ }^{\mathrm{MeCN}}=24.3\right) .{ }^{32}$ Repeating the TCC/BEMP ${ }^{1} \mathrm{H}$ NMR experiment with a deficient amount of BEMP $(2.5 \mathrm{mM})$ shows only one set of resonances for TCC, suggesting that the equilibrium in eq 1 is dynamic on the ${ }^{1} \mathrm{H}$ NMR time scale.

The ${ }^{1} \mathrm{H}$ NMR experiments suggest that TCC/BEMP would be the most imidate-like species (i.e., eq 1 further to the right) and presumably the most active TCC/organic base catalyst pair yet examined herein. Indeed, the BEMP/TCC $(0.05 \mathrm{mmol})$ catalyzed ROP of VL $(1 \mathrm{M}, 1 \mathrm{mmol})$ from benzyl alcohol $(0.01 \mathrm{mmol})$ in benzene achieves full conversion in $3 \mathrm{~min}$ (Table 3). Higher reaction concentrations can be employed, but the reaction becomes difficult to monitor, fully converting within seconds at $2 \mathrm{M} \mathrm{VL}$. The same ROP of VL fails to reach

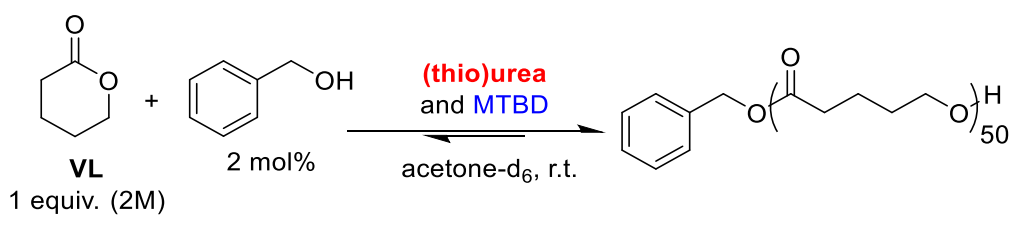

\begin{tabular}{cccccc}
\hline entry & TU or U (mol\%) & time (min) & conv. ${ }^{\mathrm{a}}(\%)$ & $M_{\mathrm{n}}{ }^{\mathrm{b}}(\mathrm{g} / \mathrm{mol})$ & $M_{\mathrm{w}} / M_{\mathrm{n}}{ }^{\mathrm{b}}$ \\
\hline 1 & TCC (5\%) & 13 & 89 & 10000 & 1.09 \\
2 & $1-\mathrm{S}(5 \%)$ & 1200 & 89 & 9500 & 1.21 \\
3 & $1-0(5 \%)$ & 60 & 91 & 11900 & 1.08
\end{tabular}




$\begin{array}{llllll}4 & \mathbf{2}-\mathbf{S}(2.5 \%) & 1020 & 90 & 11400 & 1.28 \\ 5 & \mathbf{2 - 0}(2.5 \%) & 20 & 90 & 10800 & 1.15 \\ 6 & 3-5(1.7 \%) & 7440 & 89 & 12100 & 1.16 \\ 7 & 3-0(1.7 \%) & 20 & 89 & 10300 & 1.13\end{array}$

Table 3. Urea or Thiourea Plus MTBD Cocatalyzed ROP of VL in Acetone. Reaction conditions: VL (1.0 mmol, 1 equiv, 2M), benzyl alcohol (2 mol\%), and MTBD (same mol\% as $\mathrm{U} / \mathrm{TU}$ ), acetone- $\mathrm{d}_{6}$. a) monomer conversion was monitored via ${ }^{1} \mathrm{H}$ NMR. b) $M_{\mathrm{n}}$ and $M_{\mathrm{w}} / M_{\mathrm{n}}$ were determined by GPC $\left(\mathrm{CH}_{2} \mathrm{Cl}_{2}\right)$ vs polystyrene standards.

full conversion in THF or acetone-d6 within 30 min. In $\mathrm{C}_{6} \mathrm{D}_{6}$, the ROP is highly controlled and exhibits the characteristics of a "living" polymerization (see supporting Information Figure S9), and the $[\mathrm{M}]_{0} /[]_{0}$ series (Table 3 ) is notable for the high predictability of $M_{n}$ even when considered against other organocatalytic systems. Further, $M_{\mathrm{w}} / M_{\mathrm{n}}$ broadens slowly postpolymerization (Table 3, entry 2: $3 \mathrm{~min}, M_{n}=22400, M_{w} / M_{n}=1.04 ; 6 \mathrm{~min}, M_{n}=24100, M_{w} / M_{n}=1.07 ; 15 \mathrm{~min}$, $M_{n}=24700, M_{w} / M_{n}=1.15 ; 90 \%$ conv. for all aliquots). TCC/BEMP is ineffective for the ROP of $\beta$-butyrolactone, consistent with other urea and thiourea $\mathrm{H}$-bond donors. ${ }^{13,20}$

We propose that the TCC/base cocatalyzed ROP of ester monomers proceeds through a mixed mechanism where the identity of the dominate catalyst largely depends on the $\mathrm{pK}_{\mathrm{a}}$ of the cocatalysts. The ${ }^{1} \mathrm{H}$ NMR spectrum of TCC plus $\mathrm{Me}_{6}$ TREN shows very slight downfield shift of the TCC resonances and broadening of the $\mathrm{N}-\mathrm{H}$ resonances which could be attributed to $\mathrm{H}$ bonding; there is no evidence to suggest the formation of imidate character at the urea for this cocatalyst pair (c.f. TCC/BEMP, see Supporting Information Figure S20). Accordingly, we propose that $\mathrm{TCC} /$ base cocatalyzed ROP is capable of effecting ROP through a classic dual $\mathrm{H}$ bond mechanism mediated by neutral catalysts or an imidate mediated mechanism, the primary determination of which mode is dominate rests with the $\mathrm{pK}_{\mathrm{a}}$ of the base. In the case of TCC plus Me ${ }_{6}$ TREN, we proposed a primarily neutral catalyst mechanism versus BEMP, which may proceed primarily through an imidate mechanism, Scheme 1. Certainly, the rate of the TCC/BEMP ROP recalls that of the alkoxide-generated urea anions. ${ }^{12}$ This mechanistic 


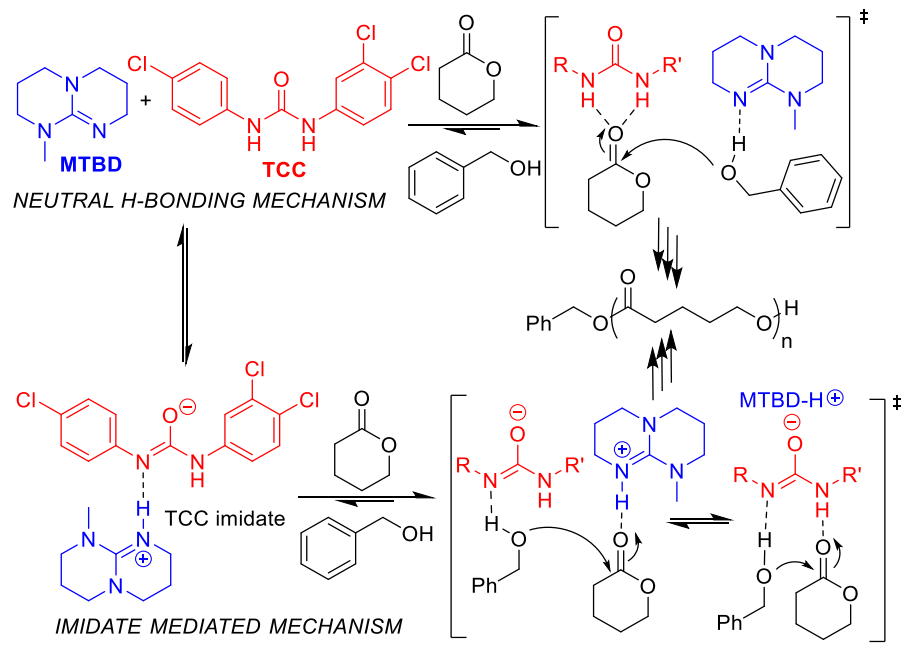

Scheme 1. Proposed mechanism for TCC/base cocatalyzed ROP.

proposal is an extension of the recent work with "hyperactive" urea anion catalysts for ROP, taking into account weakly basic cocatalysts. ${ }^{12}$ For the present system, it is unclear if the conjugate acid of the base serves as a $\mathrm{H}$-bond donor or primarily serves to deprotonate the urea. The complicated and sensitive interplay of cocatalyst/reagent interactions requires more study to be thoroughly understood.

The antibacterial TCC has been shown to be a highly effective cocatalyst for ring-opening polymerization. The commercially available $\mathrm{H}$-bond donor, when applied with an $\mathrm{H}$-bond accepting base cocatalyst, is among the most active organic catalysts for the ROP of esters, yet it exhibits the characteristics of a "living" polymerization, producing well defined polymers. The activity of this catalyst can be approximated by other mono- and dichloro biaryl urea $\mathrm{H}$ bond donor(s), which adds synthetic flexibility for the generation of future $\mathrm{H}$-bond donating ureas. We suspect that the ROP of lactone monomers is just one application that can offer new roles to old reagents, in this case, the antibacterial compound now banned in hand soap, TCC. 


\section{EXPERIMENTAL SECTION}

General Considerations. All chemicals were purchased from Fisher Scientific and used as received unless stated otherwise. Triclocarban (TCC), 7-methyl-1,5,7-triazabicyclo[4.4.0]dec5-ene (MTBD) and 1,8-diazabicyclo[5.4.0]undec-7-ene (DBU) were purchased from TCI. Tris[2(dimethylamino)ethyl]amine (Me6 TREN) and 2-tert-butylimino-2-diethylamino-1,3dimethylperhydro-1,3,2-diazaphosphorine (BEMP) were purchased from Alfa Aesar. Benzyl alcohol and 1,2-dichlorobenzene were distilled under high vacuum from calcium hydride. THF was dried on an Innovative Technology solvent purification system. DMF was dried over $4 \AA$ molecular sieves for $48 \mathrm{~h}$ prior to use. 1-pyrenebutanol was purchased from Sigma Aldrich. $\delta$ valerolactone $(\mathrm{VL})$, $\varepsilon$-caprolactone $(\mathrm{CL})$ and $\beta$-butyrolactone $(\mathrm{BL})$ were distilled from calcium hydride under high vacuum. L-Lactide (L-LA) was purchased from Acros Organics and recrystallized from dry toluene. Benzene- $d_{6}$ and chloroform- $d$ were purchased from Cambridge Isotope Laboratories and distilled from calcium hydride. Acetone- $d_{6}$ was purchased from Cambridge Isotope Laboratories and stored over $4 \AA$ molecular sieves for $48 \mathrm{~h}$ prior to use. Experiments were conducted using pre-dried glassware in an MBRAUN or INERT stainless steel glovebox or using a Schlenk line under nitrogen atmosphere. NMR experiments were conducted on a Bruker Avance III $300 \mathrm{MHz}$ or $400 \mathrm{MHz}$ spectrometer or a Varian $500 \mathrm{MHz}$ spectrometer. Gel Permeation Chromatography (GPC) was performed at $40^{\circ} \mathrm{C}$ using HPLC grade dichloromethane eluent on an Agilent Infinity GPC system equipped with three Agilent PLGel columns $7.5 \mathrm{~mm} \times 300 \mathrm{~mm}\left(5 \mu \mathrm{m}\right.$, pore sizes: $\left.10^{3}, 10^{4}, 50 \AA\right) . M_{\mathrm{n}}$ and $M_{\mathrm{w}} / M_{\mathrm{n}}$ were determined versus polystyrene standards $(500 \mathrm{~g} / \mathrm{mol}-3150 \mathrm{~kg} / \mathrm{mol}$, Polymer Laboratories). Mass spectrometry was performed using a Thermo Scientific (San Jose, CA, USA) LTQ Orbitrap $\mathrm{XL}$ mass spectrometer affixed with either an atmospheric-pressure chemical ionization (APCI) or electrospray ionization (ESI) interface, positive ions were produced and introduced into the S2 instrument. Tune conditions for infusion experiments $(5 \mu \mathrm{L} / \mathrm{min}$ flow, sample concentration $5 \mu \mathrm{g} / \mathrm{mL}$ in $50 / 50 \mathrm{v} / \mathrm{v}$ water/methanol) were as follows: ionspray voltage, $5 \mathrm{kV}$; capillary temperature, $275^{\circ} \mathrm{C}$; sheath gas (N2, arbitrary units), 8; auxiliary gas ( $\mathrm{N}_{2}$, arbitrary units), 2; capillary voltage, $35 \mathrm{~V}$; and tube lens, $90 \mathrm{~V}$. Prior to analysis, the instrument was calibrated for 
positive ions using Pierce LTQ ESI positive ion calibration solution (lot \#PC197784). Ion trap experiments used $\mathrm{N}_{2}$ as a collision gas with normalized collision energies (NCE) between 10$25 \mathrm{eV}$ for multistage fragmentation. High-energy collision (HCD) experiments were performed with $\mathrm{He}$ as the collision gas with a NCE of $25 \mathrm{eV}$.

Example ring-opening polymerization of VL. To a $7 \mathrm{~mL}$ vial, TCC (15.7 mg, $0.05 \mathrm{mmol}), \mathrm{VL}$ $(100 \mathrm{mg}, 1.0 \mathrm{mmol})$ and benzene- $d_{6}(250 \mu \mathrm{L})$ were added. The contents were stirred until the solution became homogenous. To a second $7 \mathrm{ml}$ vial, benzyl alcohol $(4.3 \mathrm{mg}, 0.04 \mathrm{mmol}$ ), MTBD $(7.6 \mathrm{mg}, 0.05 \mathrm{mmol})$ and benzene- $d_{6}(250 \mu \mathrm{L})$ were added. The contents in the second vial were transferred to the first vial via Pasteur pipette, and the contents were agitated to mix. The reaction solution was then transferred to an NMR tube, and the progress of the reaction monitored by ${ }^{1} \mathrm{H}$ NMR. The reaction was quenched by the addition of benzoic acid $(6.1 \mathrm{mg}$, $0.05 \mathrm{mmol}$ ). Polymer isolated by precipitation with hexanes contains residual TCC that can be removed by repeated precipitation or washing with methanol. PVL was removed of volatiles under high vacuum prior to characterization. Yield $89 \%, M_{n}(\mathrm{GPC})=5,400, M_{\mathrm{w}} / M_{\mathrm{n}}=1.09, M_{\mathrm{n}}$ $(\mathrm{NMR})=2,700$.

Example post-polymerization transesterification. To a $7 \mathrm{~mL}$ vial, TCC $(15.7 \mathrm{mg}, 0.05 \mathrm{mmol})$, VL $(100 \mathrm{mg}, 1.0 \mathrm{mmol})$ and benzene- $d_{6}(250 \mu \mathrm{L})$ were added. The contents were stirred until the solution became homogenous. To a second $7 \mathrm{ml}$ vial, benzyl alcohol $(1.1 \mathrm{mg}, 0.01 \mathrm{mmol})$, MTBD $(7.6 \mathrm{mg}, 0.05 \mathrm{mmol})$ and benzene- $d_{6}(250 \mu \mathrm{L})$ were added. The contents in the second vial were transferred to the first vial via Pasteur pipette, and the contents were agitated to mix. Three $50 \mu \mathrm{L}$ aliquots from the reaction were quenched at $20 \mathrm{~min}, 45 \mathrm{~min}$ and $60 \mathrm{~min}$ using benzoic acid $(6.2 \mathrm{mg}, 0.05 \mathrm{mmol})$. Polymer in each aliquot was then isolated by precipitation with hexanes. PVL was removed of volatiles under high vacuum prior to characterization by GPC: $M_{\mathrm{n}}=22,300,23,900,23,900, M_{\mathrm{w}} / M_{\mathrm{n}}=1.02,1.03,1.03$ respectively.

Example chain extension experiment. To a $7 \mathrm{~mL}$ vial, TCC $(15.7 \mathrm{mg}, 0.05 \mathrm{mmol}), \mathrm{VL}(100$ $\mathrm{mg}, 1.0 \mathrm{mmol})$ and benzene- $d_{6}(250 \mu \mathrm{L})$ were added. The contents were agitated until the solution became homogenous. To a second $7 \mathrm{ml}$ vial, 1-pyrenebutanol (5.5 mg, $0.02 \mathrm{mmol}$ ), MTBD $(7.6 \mathrm{mg}, 0.05 \mathrm{mmol})$ and benzene- $d_{6}(250 \mu \mathrm{L})$ were added. The contents of the second 
vial were transferred to the first vial via Pasteur pipette, and the contents were agitated to mixed. After $13 \mathrm{~min}$, a $100 \mu \mathrm{L}$ aliquot from the reaction was quenched using benzoic acid $(6.2 \mathrm{mg}, 0.05$ $\mathrm{mmol})$, and $\mathrm{VL}(100 \mathrm{mg}, 1.0 \mathrm{mmol})$ was added to the reaction vial. A second $100 \mu \mathrm{L}$ aliquot from the reaction vial was quenched in $27 \mathrm{~min}$ using benzoic acid $(6.2 \mathrm{mg}, 0.05 \mathrm{mmol})$. Conversion of $\mathrm{VL}$ in the two aliquots were then determined by ${ }^{1} \mathrm{H} \mathrm{NMR}$, followed by the isolation of PVL and characterization by GPC.

Example ring-opening polymerization of L-Lactide. A first $7 \mathrm{~mL}$ vial was charged with TCC (15.8 mg, $0.05 \mathrm{mmol}), \mathrm{Me}_{6} \operatorname{TREN}(13.4 \mu \mathrm{L}, 0.05 \mathrm{mmol}$ ) and benzyl alcohol (1.0 $\mu \mathrm{L}, 0.01 \mathrm{mmol})$. A second $7 \mathrm{~mL}$ vial was charged with L-LA $(144.1 \mathrm{mg}, 1 \mathrm{mmol})$ and acetone- $d_{6}(1000 \mu \mathrm{L})$. The contents of the second vial were added to the first vial, and the resulting mixture was vigorously shaken until homogenous. The reaction mixture was transferred to an NMR tube via pipette, and the reaction progress was monitored by ${ }^{1} \mathrm{H}$ NMR. The reaction was quenched with benzoic acid $(0.1 \mathrm{mmol})$. The reaction mixture was removed of volatiles under reduced pressure, dissolved in minimal dichloromethane, and the polylactide (PLA) was precipitated with the addition of hexanes. The supernatant was decanted, and the precipitate was subjected to high vacuum to remove volatiles.

Example binding experiment. For the titration of 1-O with $\mathrm{CL}$, stock solutions of 1-O and $\mathrm{CL}$ were prepared in benzene- $d_{6}$. Into several NMR tubes, varying amounts of each solution were added to each tube along with neat benzene- $d_{6}$ such that the final volume of each sample was $0.4 \mathrm{~mL}$. The final concentrations were $[1-0]=0.005 \mathrm{M}$ and $0.25 \mathrm{M}<[\mathrm{CL}]<2.25 \mathrm{M}$. ${ }^{1} \mathrm{H}-\mathrm{NMR}$ spectra (referenced to residual benzene- $\mathrm{H}$ ) were acquired for each tube at $300 \mathrm{~K}$ and the chemical shift of the ortho-protons of 1-0 was noted. Binding constants were determined by the curve fitting method, ${ }^{33-35}$ and these values match those determined from the LineweaverBurke method. ${ }^{36,37}$ Binding curves are shown below (see Supporting Information Figures S. Example synthesis of 1-(4-chlorophenyl)-3-phenylurea (mono-CC). A dried Schlenk flask was charged with 4-chlorophenylisocyanate $(598.2 \mathrm{mg}, 3.90 \mathrm{mmol})$ and $\sim 10 \mathrm{~mL}$ dried DCM. Next, aniline $(0.36 \mathrm{~mL}, 3.95 \mathrm{mmol})$ was added via syringe. Immediately upon addition of aniline, a white precipitate formed. The reaction mixture was filtered and rinsed 3 times with cold DCM 
to provide a pure white powder $(846.1 \mathrm{mg}, 3.43 \mathrm{mmol}, 88.1 \%$ yield). Characterization matches literature; ${ }^{38} \mathrm{NMR}$ spectra below; ${ }^{13} \mathrm{C} \mathrm{NMR}\left(\mathrm{CD}_{3} \mathrm{OD}, 100 \mathrm{MHz}\right): \delta=155.2,140.3,139.5,129.5$, $129.4,128.5,124.0,121.6,120.5$.

Example synthesis of 1-(3,4-dichlorophenyl)-3-phenylurea (di-CC). A dried Schlenk flask was charged with 3,4-dichlorophenylisocyanate $(731.8 \mathrm{mg}, 3.89 \mathrm{mmol})$ and $\sim 10 \mathrm{~mL}$ dried DCM. Next, aniline $(0.36 \mathrm{~mL}, 3.95 \mathrm{mmol})$ was added via syringe. Immediately upon addition of aniline a white precipitate formed. The reaction mixture was filtered and rinsed 3 times with cold DCM to provide a pure white powder $(1.01 \mathrm{~g}, 3.59 \mathrm{mmol}, 92.7 \%$ yield). Characterization matches literature; ${ }^{39} \mathrm{NMR}$ spectra below; ${ }^{13} \mathrm{C} \mathrm{NMR}\left(\mathrm{CD}_{3} \mathrm{OD}, 100 \mathrm{MHz}\right): \delta=154.87,140.81,140.16$, 133.32, 131.48, 129.89, 126.14, 124.12, 121.39, 120.53, 119.60. 


\section{AKNOWLEGDEMENTS}

The authors declare no competing financial interest. This research was supported by an NSF CAREER Award (CHE 1554830) and the University of Rhode Island. This research features data acquired on a $400 \mathrm{MHz}$ NMR spectrometer purchased thanks to the support of the NSF (1531963). We wish to thank Fredrik Haeffner (Boston College) for his mechanistic insights. 


\section{LIST OF REFERENCES}

(1) Kiesewetter, M. K.; Shin, E. J.; Hedrick, J. L.; Waymouth, R. M. Organocatalysis: Opportunities and Challenges for Polymer Synthesis. Macromolecules 2010, 43, 2093-2107.

(2) Kamber, N. E.; Jeong, W.; Waymouth, R. M.; Pratt, R. C.; Lohmeijer, B. G. G.; Hedrick, J. L. Organocatalytic Ring-Opening Polymerization. Chem. Rev. 2007, 107, 5813-5840.

(3) Pratt, R. C.; Lohmeijer, B. G. G.; Long, D. A.; Lundberg, P. N. P.; Dove, A. P.; Li, H.; Wade, C. G.; Waymouth, R. M.; Hedrick, J. L. Exploration, Optimization, and Application of Supramolecular Thiourea-Amine Catalysts for the Synthesis of Lactide (Co)polymers. Macromolecules 2006, 39, 7863-7871.

(4) Kazakov, O. I.; Datta, P. P.; Isajani, M.; Kiesewetter, E. T.; Kiesewetter, M. K. Cooperative Hydrogen-Bond Pairing in Organocatalytic Ring-Opening Polymerization. Macromolecules 2014, 47, 7463-7468.

(5) Csihony, S.; Culkin, D. A.; Sentman, A. C.; Dove, A. P.; Waymouth, R. M.; Hedrick, J. L. Single-Component Catalyst/initiators for the Organocatalytic Ring-Opening Polymerization of Lactide. J. Am. Chem. Soc. 2005, 127, 9079-9084.

(6) McKinlay, C. J.; Waymouth, R. M.; Wender, P. A. Cell-Penetrating, Guanidinium-Rich Oligophosphoesters: Effective and Versatile Molecular Transporters for Drug and Probe Delivery. J. Am. Chem. Soc. 2016, 138, 3510-3517.

(7) Blake, T. R.; Waymouth, R. M. Organocatalytic Ring-Opening Polymerization of Morpholinones: New Strategies to Functionalized Polyesters. J. Am. Chem. Soc. 2014, $136,9252-9255$.

(8) Miyake, G. M.; Chen, E. Y. X. Cinchona Alkaloids as Stereoselective Organocatalysts for the Partial Kinetic Resolution Polymerization of Rac-Lactide. Macromolecules 2011, $44,4116-4124$.

(9) (a) Naumann, S.; Scholten, P. B. V; Wilson, J. A.; Dove, A. P. Dual Catalysis for Selective Ring-Opening Polymerization of Lactones: Evolution toward Simplicity. J. Am. 
Chem. Soc. 2015, 137, 14439-14445.(b) Xu, S.; Sun, H.; Liu, J.; Xu, J.; Pan, X.; Dong, H.; Liu, Y.; Li, Z.; Guo, K. Internal Lewis pair enhanced H-bond donor: boronate-urea and tertiary amine co-catalysis in ring-opening polymerization. Polym. Chem. 2016, 7, 6843.

(10) Zhang, X.; Jones, G. O.; Hedrick, J. L.; Waymouth, R. M. Fast and Selective RingOpening Polymerizations by Alkoxides and Thioureas. Nat. Chem. 2016, 8, 1047-1053.

(11) Guillaume, S. M.; Kirillov, E.; Sarazin, Y.; Carpentier, J. F. Beyond Stereoselectivity, Switchable Catalysis: Some of the Last Frontier Challenges in Ring-Opening Polymerization of Cyclic Esters. Chem. - Eur. J. 2015, 21, 7988-8003.

(12) Lin, B.; Waymouth, R. M. Urea Anions: Simple, Fast, and Selective Catalysts for RingOpening Polymerizations. J. Am. Chem. Soc. 2017, 139, 1645-1652.

(13) Fastnacht, K. V.; Spink, S. S.; Dharmaratne, N. U.; Pothupitiya, J. U.; Datta, P. P.; Kiesewetter, E. T.; Kiesewetter, M. K. Bis- and Tris-Urea H-Bond Donors for RingOpening Polymerization: Unprecedented Activity and Control from an Organocatalyst. ACS Macro Lett. 2016, 5, 982-986.

(14) Kolb, H. C.; Finn, M. G.; Sharpless, K. B. Click Chemistry: Diverse Chemical Function from a Few Good Reactions. Angew. Chem., Int. Ed. 2001, 40, 2004-2021.

(15) Dove, A. P.; Pratt, R. C.; Lohmeijer, B. G. G.; Waymouth, R. M.; Hedrick, J. L. ThioureaBased Bifunctional Organocatalysis: Supramolecular Recognition for Living Polymerization. J. Am. Chem. Soc. 2005, 127, 13798-13799.

(16) Erickson, B. E. FDA Bans Antibacterials in Consumers Soaps. Chem. Eng. News 2016, $94,16$.

(17) DeLeo, P. C.; Sedlak, R. I. Comment on "On the Need and Speed of Regulating Triclosan and Triclocarban in the United States. Environ. Sci. Technol. 2014, 48, $11021-11022$.

(18) Pycke, B. F. G.; Roll, I. B.; Brownawell, B. J.; Kinney, C. A.; Furlong, E. T.; Kolpin, D. W.; Halden, R. U. Transformation Products and Human Metabolites of Triclocarban and 
Triclosan in Sewage Sludge across the United States. Environ. Sci. Technol. 2014, 48, 7881-7890.

(19) Pycke, B. F. G.; Geer, L. A.; Dalloul, M.; Abulafia, O.; Jenck, A. M.; Halden, R. U. Human Fetal Exposure to Triclosan and Triclocarban in an Urban Population from Brooklyn, New York. Environ. Sci. Technol. 2014, 48, 8831-8838.

(20) Doyle, A. G.; Jacobsen, E. N. Small-Molecule H-Bond Donors in Asymmetric Catalysis. Chem. Rev. 2007, 107, 5713-5743.

(21) Kazakov, O. I.; Kiesewetter, M. K. Cocatalyst Binding Effects in Organocatalytic RingOpening Polymerization of L-Lactide. Macromolecules 2015, 48, 6121-6126.

(22) Coady, D. J.; Engler, A. C.; Horn, H. W.; Bajjuri, K. M.; Fukushima, K.; Jones, G. O.; Nelson, A.; Rice, J. E.; Hedrick, J. L. Catalyst Chelation Effects in Organocatalyzed Ring-Opening Polymerization of Lactide. ACS Macro Lett. 2012, 1, 19-22.

(23) Spink, S. S.; Kazakov, O. I.; Kiesewetter, E. T.; Kiesewetter, M. K. Rate Accelerated Organocatalytic Ring-Opening Polymerization of L-Lactide via the Application of a Bis(thiourea) H-Bond Donating Cocatalyst. Macromolecules 2015, 48, 6127-6131.

(24) Lohmeijer, B. G. G.; Pratt, R. C.; Leibfarth, F.; Logan, J. W.; Long, D. A.; Dove, A. P.; Nederberg, F.; Choi, J.; Wade, C.; Waymouth, R. M.; Hedrick, J. L. Guanidine and Amidine Organocatalysts for Ring-Opening Polymerization of Cyclic Esters. Macromolecules 2006, 39, 8574-8583.

(25) Kulkarni, A. R.; Garai, S.; Thakur, G. A. Scalable, One-Pot, Microwave-Accelerated Tandem Synthesis of Unsymmetrical Urea Derivatives. J. Org. Chem. 2017, 82, 992.

(26) Beaver, D. J.; Roman, D. P.; Stoffel, P. J. The Preparation and Bacteriostatic Activity of Substituted Ureas. J. Am. Chem. Soc. 1957, 79, 1236-1245.

(27) Lippert, K. M.; Hof, K.; Gerbig, D.; Ley, D.; Hausmann, H.; Guenther, S.; Schreiner, P. R. Hydrogen-Bonding Thiourea Organocatalysts: The Privileged 3,5Bis(trifluoromethyl)phenyl Group. Eur. J. Org. Chem. 2012, 30, 5919-5927.

(28) Zhang, Z.; Schreiner, P. R. (Thio)urea Organocatalysis-What Can Be Learnt from Anion Recognition? Chem. Soc. Rev. 2009, 38, 1187-1198. 
(29) Datta, P. P.; Kiesewetter, M. K. Controlled Organocatalytic Ring-Opening Polymerization of $\varepsilon$-Thionocaprolactone. Macromolecules 2016, 49, 774-780.

(30) Bannin, T. J.; Kiesewetter, M. K. Poly(thioester) by Organocatalytic Ring-Opening Polymerization. Macromolecules 2015, 48 (16), 5481-5486.

(31) Schwesinger, R.; Schlempep, H.; Hasenfratz, C.; Willaredt, J.; Dambacher, T.; Breuer, T.; Ottaway, C.; Fletschinger, M.; Boele, J.; Fritz, H.; Putzas, D.; Rotter, H. W.; Bordwell, F. G.; Satish, A. V.; Ji, G.; Peters, E.; Peters, K.; Schnering, H. G. V.; Walz, L. Extremely Strong, Uncharged Auxiliary Bases; Monomeric and Polymer-Supported Polyaminophosphazenes (P2-P5). Liebigs Ann. 1996, 1996, 1055-1081.

(32) Kaljurand, I.; Kütt, A.; Sooväli, L.; Rodima, T.; Mäemets, V.; Leito, I.; Koppel, I. A. Extension of the Self-Consistent Spectrophotometric Basicity Scale in Acetonitrile to a Full Span of 28 pKa Units: Unification of Different Basicity Scales. J. Org. Chem. 2005, 70, 1019-1028.

(33) Webb, J. E. A.; Crossley, M. J.; Turner, P.; Thordarson, P. Pyromellitamide Aggregates and Their Response to Anion Stimuli. J. Am. Chem. Soc. 2007, 129, 7155.

(34) Thordarson, P. Determining association constants from titration experiments in supramolecular chemistry. Chem. Soc. Rev. 2011, 40, 1305.

(35) Deranleau, D. A. Theory of the measurement of weak molecular complexes. II. Consequences of multiple equilibria. J. Am. Chem. Soc. 1969, 91, 4044.

(36) Horman, I.; Dreux, B. Estimation of association constants of bimolecular complexes. Reply to comments. Anal. Chem. 1983, 55, 1219.

(37) Peters, S. J.; Stevenson, C. D. The Complexation of Na+ by 18-crown -6 studied via nuclear magnetic resonance. J. Chem. Educ. 2004, 81, 715.

(38) Lee, H-G.; Kim, M-J.; Park, S-E.; Kin, J-J.; Kim, B. R.; Lee, S-G.; Yoon, Y-J. Phenyl 4,5dichloro-6-oxopyridazine-1(6H)-carboxylate as carbonyl source: facile and selective synthesis of carbamates and ureas under mild conditions. Synlett 2009, 17, 2809. 
(39) Khan, K. M.; Saeed, S.; Ali, M.; Gohar, M.; Zahid, J.; Khan, A.; Perveen, S.; Choudhary, M. I. Unsymmetrically disubstituted urea derivatives: A potent class of antiglycating agents. Bioorg. Med. Chem. 2009, 17, 2447. 


\section{SUPPORTING INFORMATION: Triclocarban: Commercial Antibacterial and Highly Effective H-Bond Donating Catalyst for Ring-Opening Polymerization}

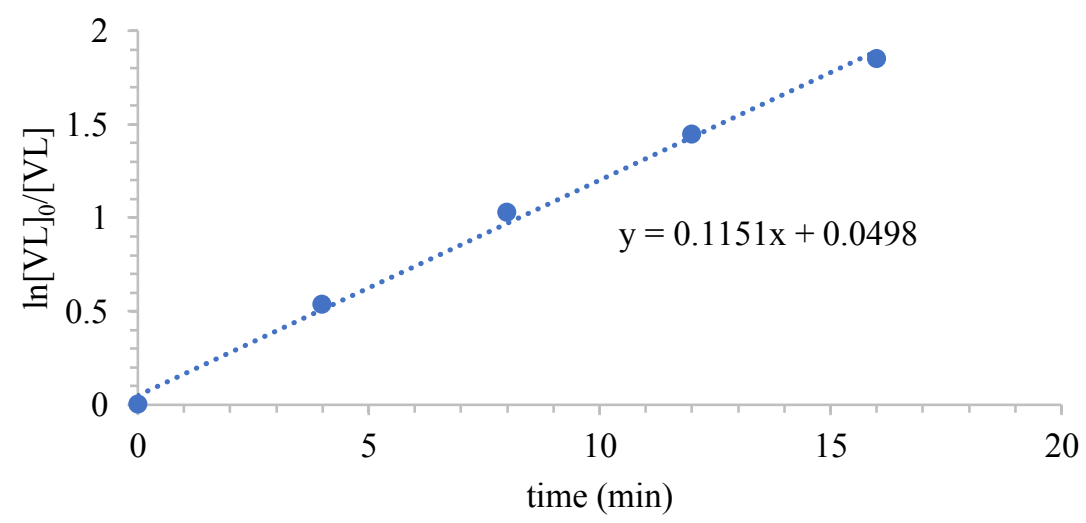

Figure 2. First order evolution of VL vs time for the TCC/MTBD catalyzed ring-opening polymerization of VL. Conditions: VL (2 M, $1 \mathrm{mmol})$, benzyl alcohol (1mol\%, $0.01 \mathrm{mmol})$, TCC (5mol\%, $0.05 \mathrm{mmol})$, MTBD (5 mol\%, $0.05 \mathrm{mmol}$ ) in benzene- $d_{6}$.

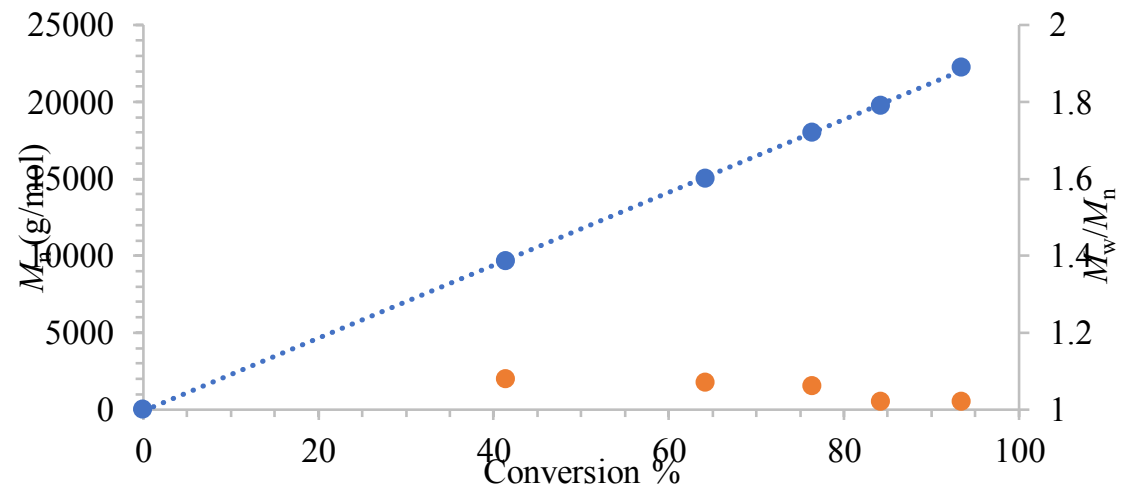

Figure 3. $M_{\mathrm{n}}$ (blue) and $M_{\mathrm{w}} / M_{\mathrm{n}}$ (orange) vs conversion for the TCC/MTBD catalyzed ringopening polymerization of VL. Conditions: VL $(2 \mathrm{M}, 1 \mathrm{mmol})$, benzyl alcohol ( $1 \mathrm{~mol} \%, 0.01$ $\mathrm{mmol})$, TCC (5mol\%, $0.05 \mathrm{mmol})$, MTBD $(5 \mathrm{~mol} \%, 0.05 \mathrm{mmol})$ in benzene- $d_{6}$. 


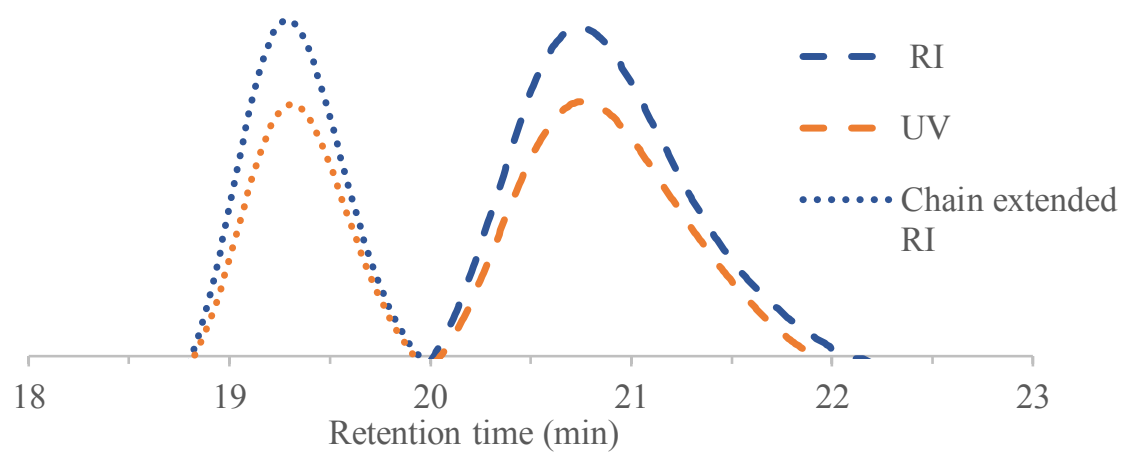

Figure 4. GPC traces of the polymers resulting from the chain extension experiment of VL. Conditions: VL (2 M, 1 mmol), 1-pyrenebutanol (2mol\%, 0.02mmol), TCC ( $5 \mathrm{~mol} \%, 0.05 \mathrm{mmol})$, MTBD (5 mol\%, $0.05 \mathrm{mmol})$ in benzene- $d_{6}$, and subsequent chain extension by the addition of $\mathrm{VL}(1 \mathrm{mmol})$.

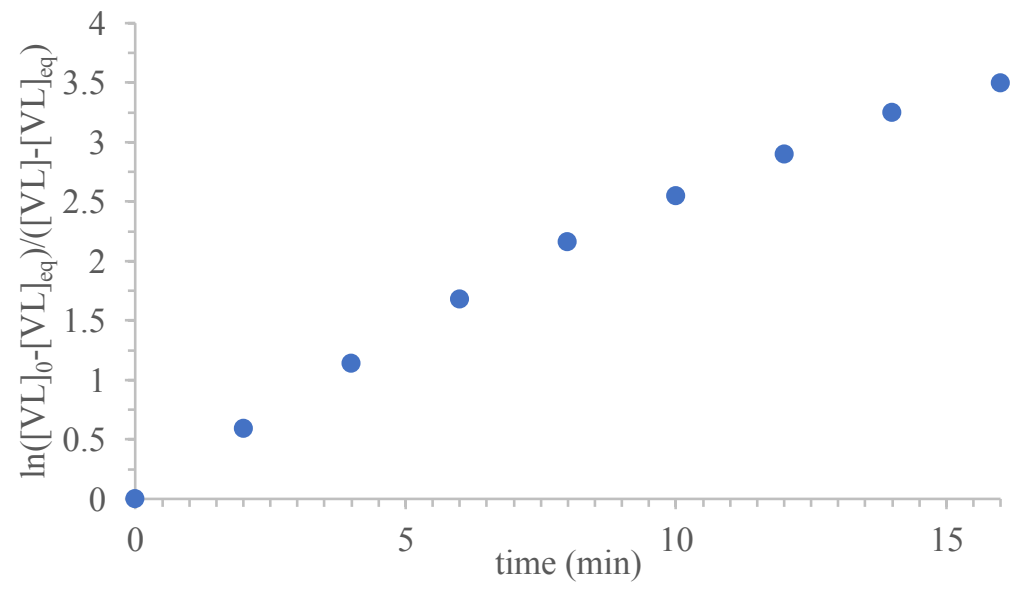

Figure 5. Approach to equilibrium evolution of [VL] vs time for the TCC/MTBD catalyzed ringopening polymerization of VL. Conditions: VL (2.1 M, $2 \mathrm{mmol}, 1$ equiv.), benzyl alcohol $(1 \mathrm{~mol} \%, 0.02 \mathrm{mmol})$, TCC $(5 \mathrm{~mol} \%, 0.1 \mathrm{mmol})$, MTBD $(5 \mathrm{~mol} \%, 0.1 \mathrm{mmol})$ in acetone- $d_{6}$. $[\mathrm{VL}]_{\mathrm{eq}}=0.22 \mathrm{M}$. 


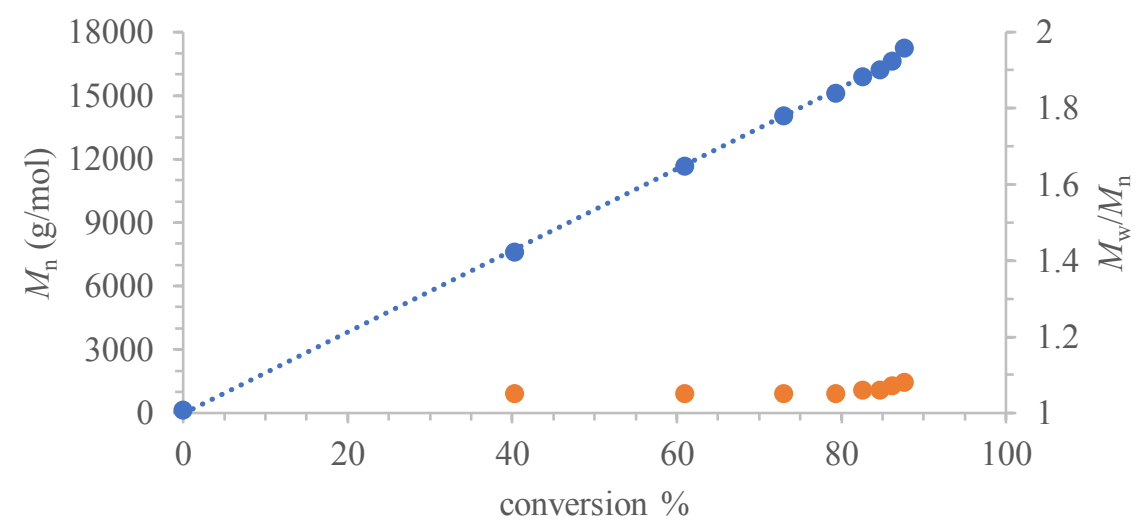

Figure 6. $M_{\mathrm{n}}$ (blue) and $M_{\mathrm{w}} / M_{\mathrm{n}}$ (orange) vs conversion for the TCC/MTBD catalyzed ringopening polymerization of VL. Conditions: VL $(2.1 \mathrm{M}, 2 \mathrm{mmol}, 1$ equiv.), benzyl alcohol (1 mol. \%, $0.02 \mathrm{mmol}$ ), TCC (5 mol. \%, $0.1 \mathrm{mmol})$, MTBD (5 mol. \%, $0.1 \mathrm{mmol})$ in acetone- $d_{6}$.

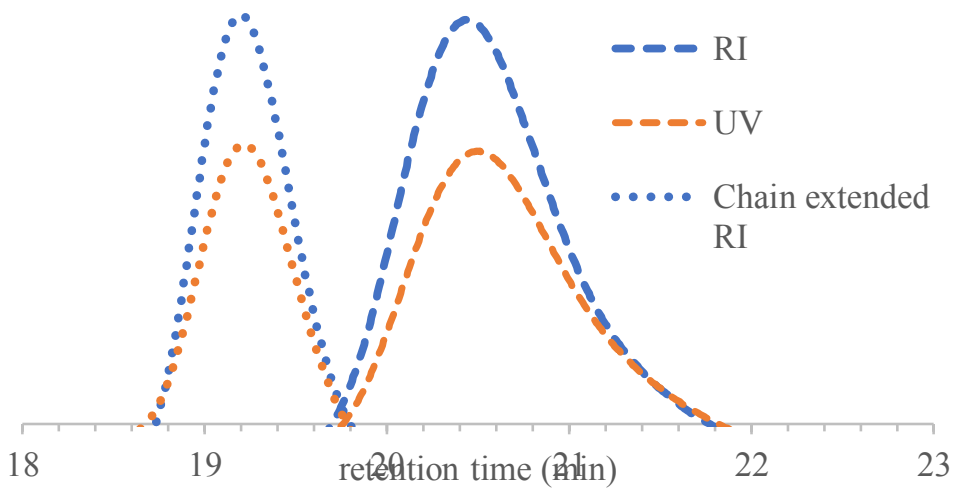

Figure 7. GPC traces of the polymers resulting from the chain extension of PVL in acetone. Conditions: VL (2 M, $1 \mathrm{mmol}), 1$-pyrenebutanol ( $2 \mathrm{~mol} \%, 0.02 \mathrm{mmol})$, TCC ( $5 \mathrm{~mol} \%, 0.05$ $\mathrm{mmol})$, MTBD ( $5 \mathrm{~mol} \%, 0.05 \mathrm{mmol})$ in acetone- $d_{6}$, and subsequent chain extension by the addition of VL (1 mmol).

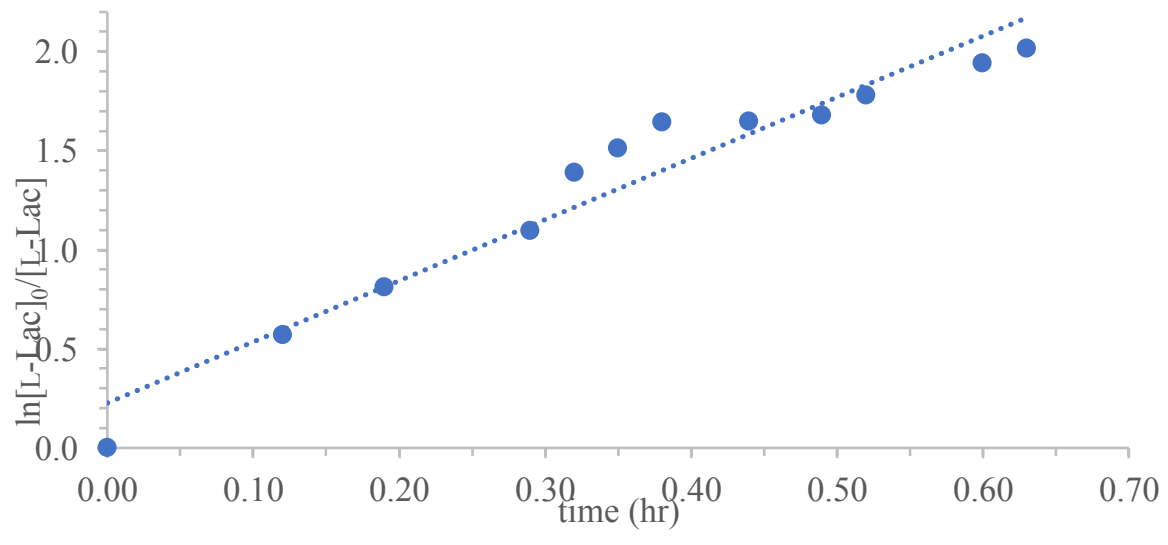


Figure 8. First order evolution of [L-LA] vs time for the TCC/Me6 TREN catalyzed ring-opening polymerization. Conditions: L-LA ( $1 \mathrm{M}, 1 \mathrm{mmol})$, benzyl alcohol $(1 \mathrm{~mol} \%, 0.01 \mathrm{mmol})$, TCC (5 $\mathrm{mol} \%, 0.05 \mathrm{mmol})$, Me 6 TREN (5 mol \%, $0.05 \mathrm{mmol})$ in acetone- $d_{6}$.

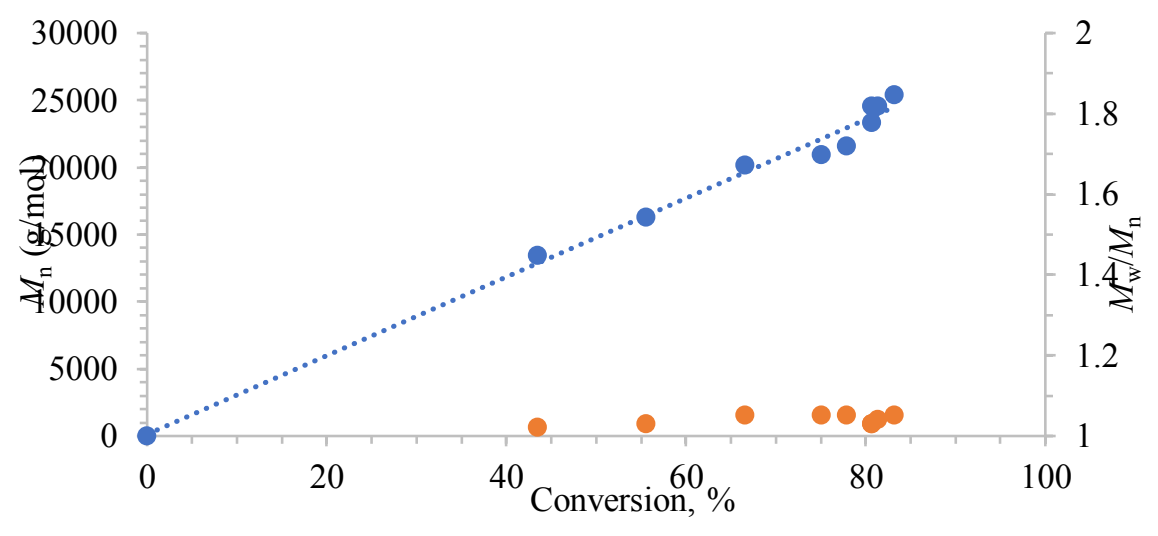

Figure 9. Mn (blue) and Mw/Mn (orange) vs conversion for the TCC/Me6TREN catalyzed ringopening polymerization of L-LA. Conditions: L-LA ( $1 \mathrm{M}, 1 \mathrm{mmol})$, benzyl alcohol (1 mol. \%, 0.01 mmol), TCC (5 mol. \%, $0.05 \mathrm{mmol}), \mathrm{Me}_{6} \operatorname{TREN}(5 \mathrm{~mol} \%$, $0.05 \mathrm{mmol})$ in acetone- $d_{6}$. 


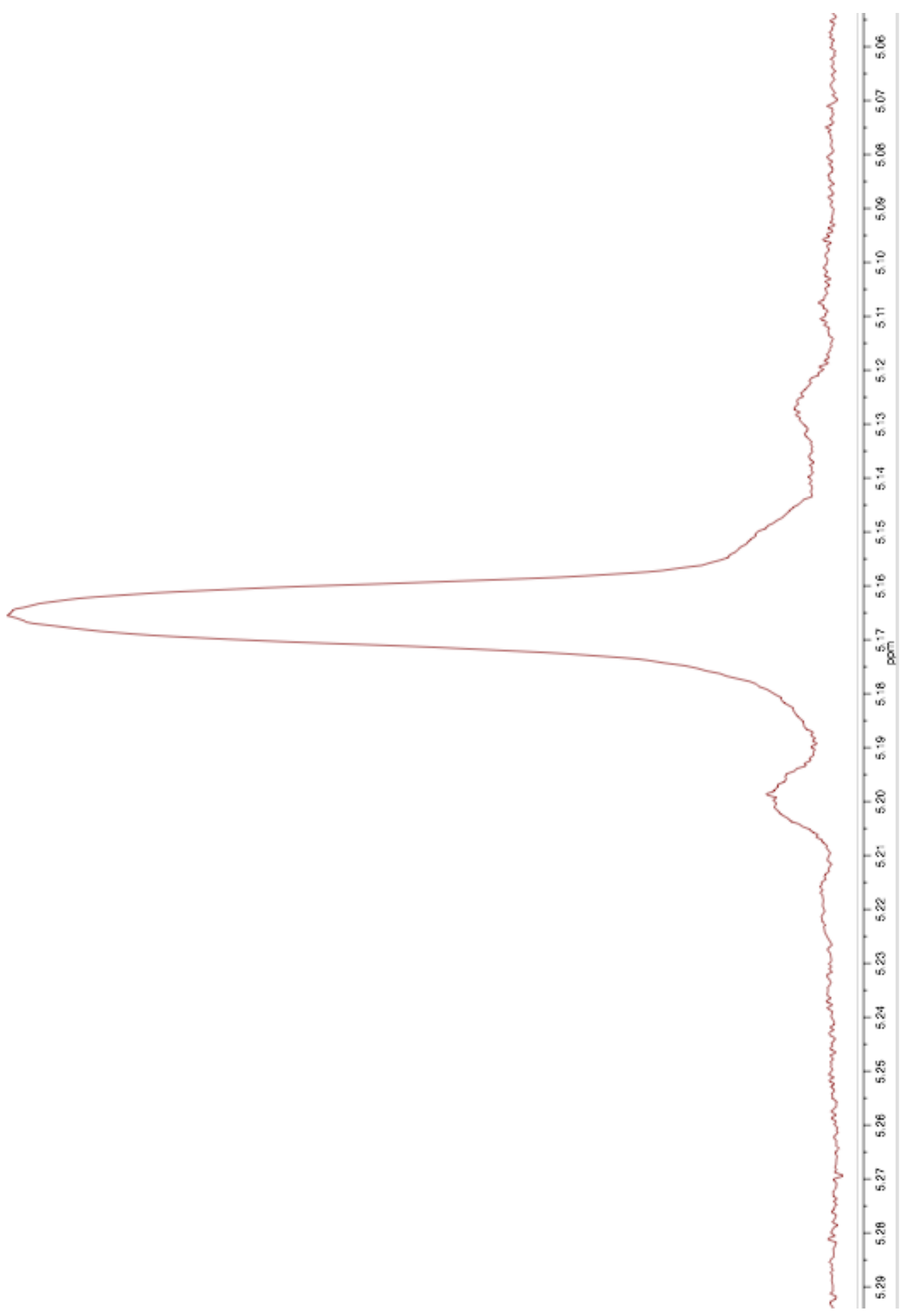

Figure 10. Methine region of the methyl-decoupled ${ }^{1} \mathrm{H}$ NMR spectrum of PLLA obtained via TCC/Me 6 TREN cocatalyzed ROP of L-LA $\left(500 \mathrm{MHz}, 25^{\circ} \mathrm{C}\right)$. 

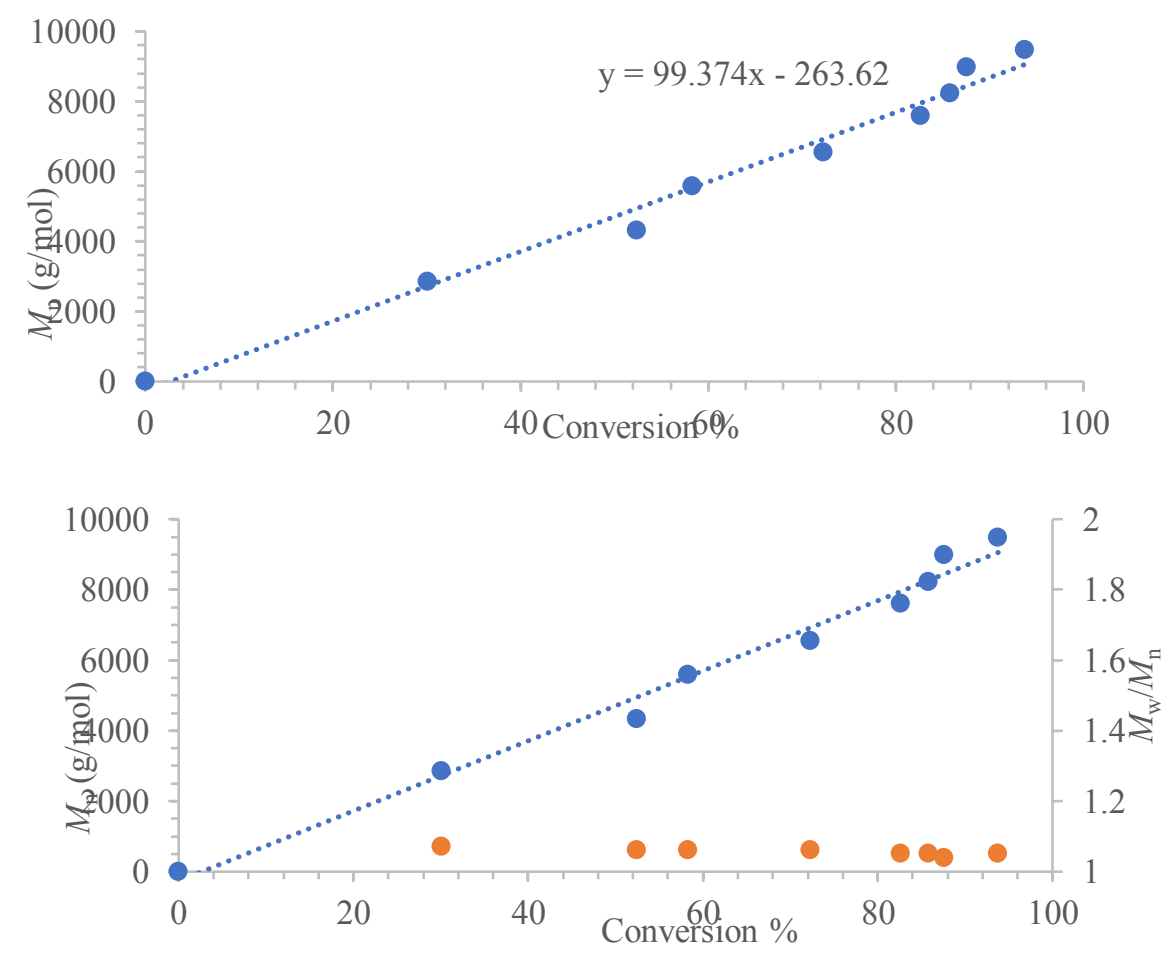

Figure 11. (upper) First order evolution of [VL] vs time for the di-CC/MTBD catalyzed ROP of VL. (lower) The ROP displays a linear evolution of $M_{\mathrm{n}}$ (blue) vs conversion and narrow $M_{\mathrm{w}} / M_{\mathrm{n}}$ (orange). Conditions:. VL (2 M, $1.0 \mathrm{mmol})$, benzyl alcohol $(2.0 \mathrm{~mol} \%, 0.02 \mathrm{mmol})$, di-CC (5.0 $\mathrm{mol} \%, 0.05 \mathrm{mmol}$ ), MTBD (5 mol\%, $0.05 \mathrm{mmol}$ ) in benzene- $d_{6}$. 


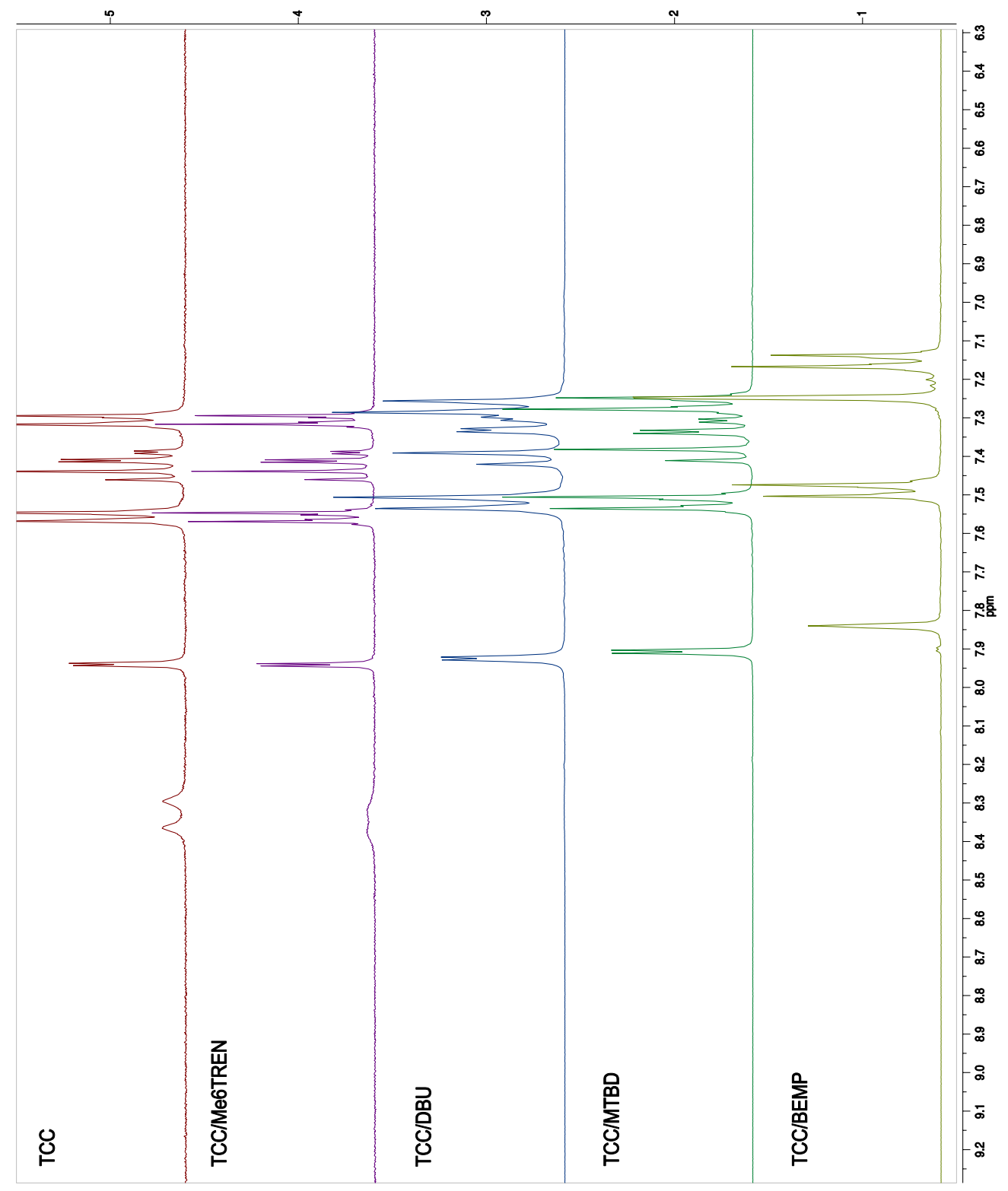

Figure 12. Downfield portion of the ${ }^{1} \mathrm{H}$ NMR spectra of TCC plus base $([T C C]=[$ base $]=5$ $\mathrm{mM})$ in acetone- $d_{6}$. 


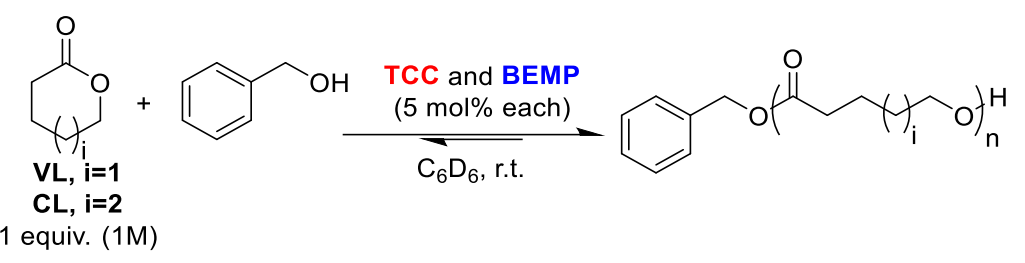

\begin{tabular}{ccccccc} 
entry & mon. & {$[\mathrm{M}] \mathrm{o} /[\mathrm{l}] \mathrm{o}$} & time $(\mathrm{min})$ & conv. $^{\mathrm{a}}(\%)$ & $M_{\mathrm{n}}^{\mathrm{b}}(\mathrm{g} / \mathrm{mol})$ & $M_{\mathrm{w}} / M_{\mathrm{n}}^{\mathrm{b}}$ \\
\hline 1 & $\mathrm{VL}$ & 50 & 1 & 87 & 11900 & 1.04 \\
2 & & 100 & 3 & 90 & 22400 & 1.04 \\
3 & & 200 & 6 & 90 & 47900 & 1.06 \\
4 & & 500 & 10 & 90 & 108800 & 1.05 \\
$5^{\mathrm{d}}$ & $\mathrm{CL}$ & 100 & 6 & 90 & 16500 & 1.04
\end{tabular}

Triclocarban Plus BEMP Cocatalyzed ROP of VL and CL. Reaction conditions: VL or CL (1.0 mmol, 1 equiv, $1 \mathrm{M}$ ), benzyl alcohol, $\mathrm{C}_{6} \mathrm{D}_{6}$. a) monomer conversion was monitored via ${ }^{1} \mathrm{H}$ NMR. b) $M_{\mathrm{n}}$ and $M_{\mathrm{w}} / M_{\mathrm{n}}$ were determined by $\mathrm{GPC}\left(\mathrm{CH}_{2} \mathrm{Cl}_{2}\right)$ vs polystyrene standards. d) $\mathrm{CL}$ (1.0 mmol, 1 equiv, 2M).

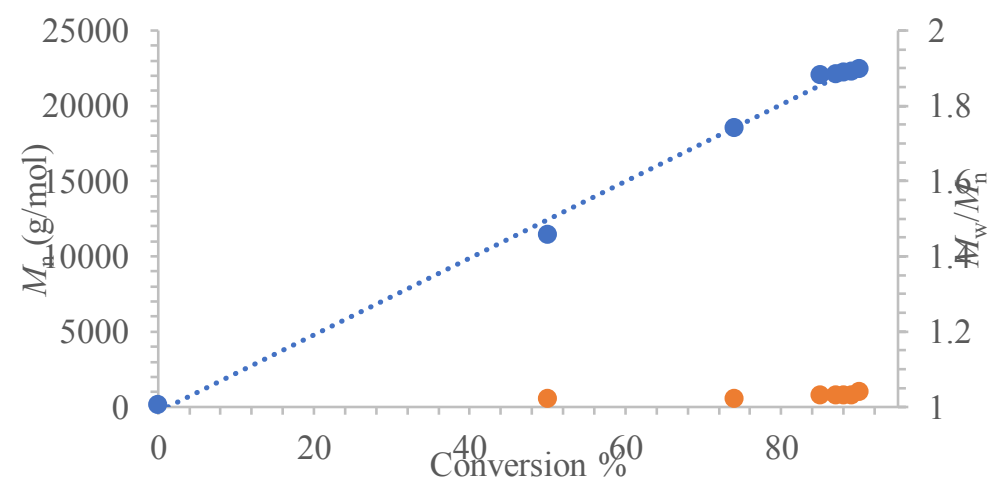

Figure 13. $M_{\mathrm{n}}$ (blue) and $M_{\mathrm{w}} / M_{\mathrm{n}}$ (orange) vs conversion for the TCC/BEMP catalyzed ringopening polymerization of VL. Conditions: VL ( $1 \mathrm{M}, 1 \mathrm{mmol})$, benzyl alcohol ( $1 \mathrm{~mol} \%, 0.01$ mmol), TCC (5 mol\%, $0.05 \mathrm{mmol})$, BEMP (5 mol\%, $0.05 \mathrm{mmol})$ in benzene- $d_{6}$. 


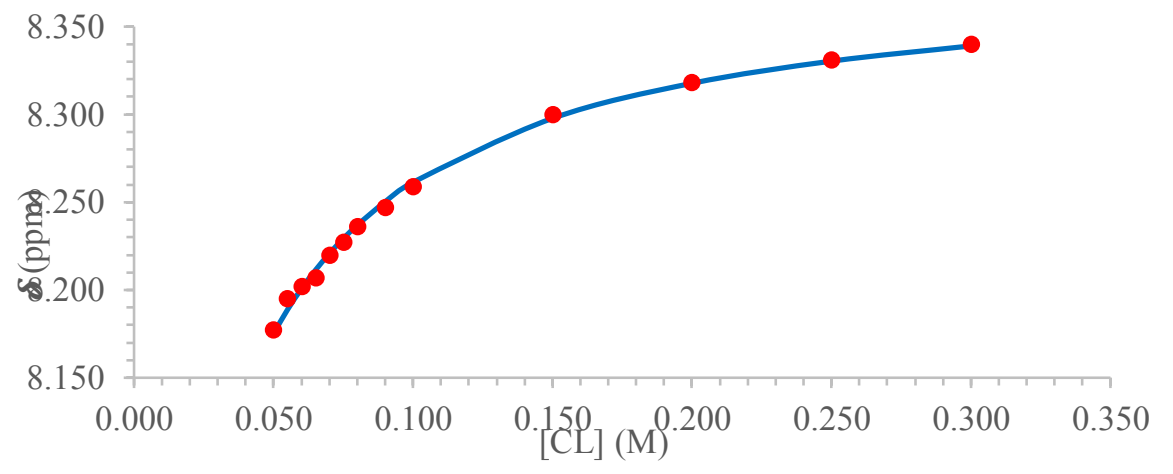

Figure 14. Titration binding curve for the CL/1-O binding in benzene- $d_{6}$. Chemical shift of the o-phenyl protons vs [CL]; solid line is the fit from the binding equation.

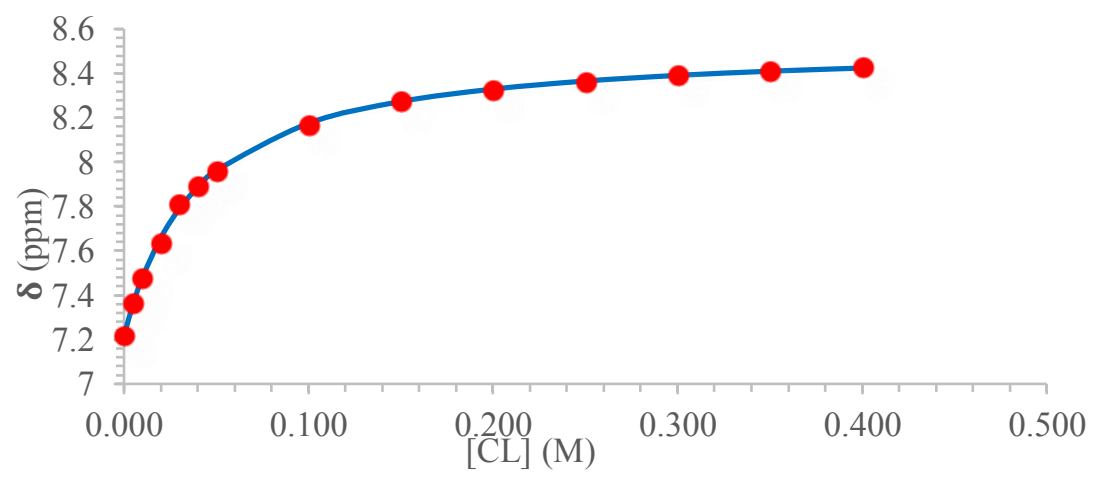

Figure 15. Titration binding curve for the CL/1-S binding in benzene- $d_{6}$. Chemical shift of the $o$-phenyl protons vs [CL]; solid line is the fit from the binding equation. 


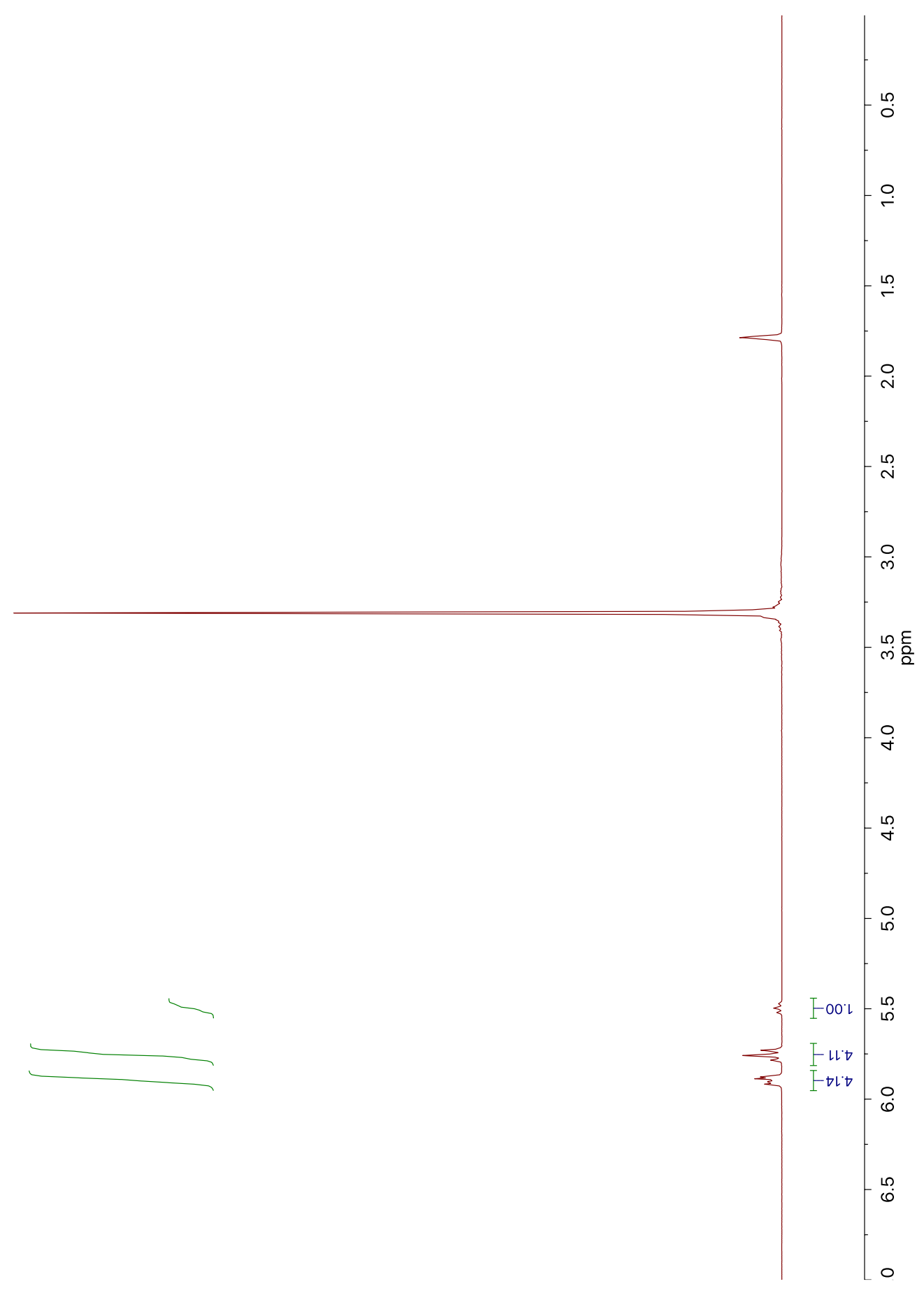

Figure 16. ${ }^{1} \mathrm{H} \mathrm{NMR}\left(\mathrm{CD}_{3} \mathrm{OD}, 400 \mathrm{MHz}\right)$ spectrum of mono-CC. 


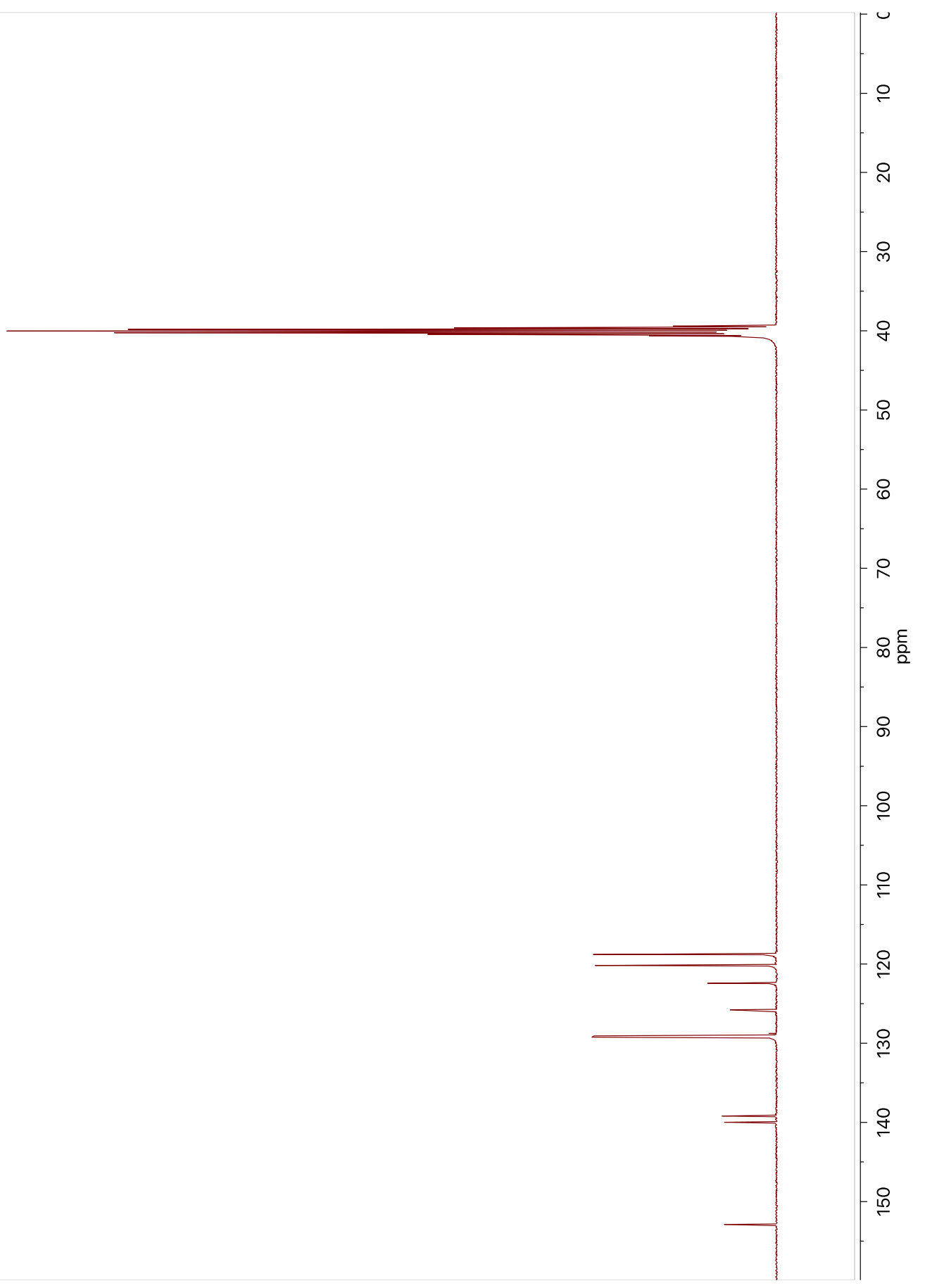

Figure 17. ${ }^{13} \mathrm{C}$ NMR $\left(\mathrm{CD}_{3} \mathrm{OD}, 100 \mathrm{MHz}\right)$ spectrum of mono-CC. 


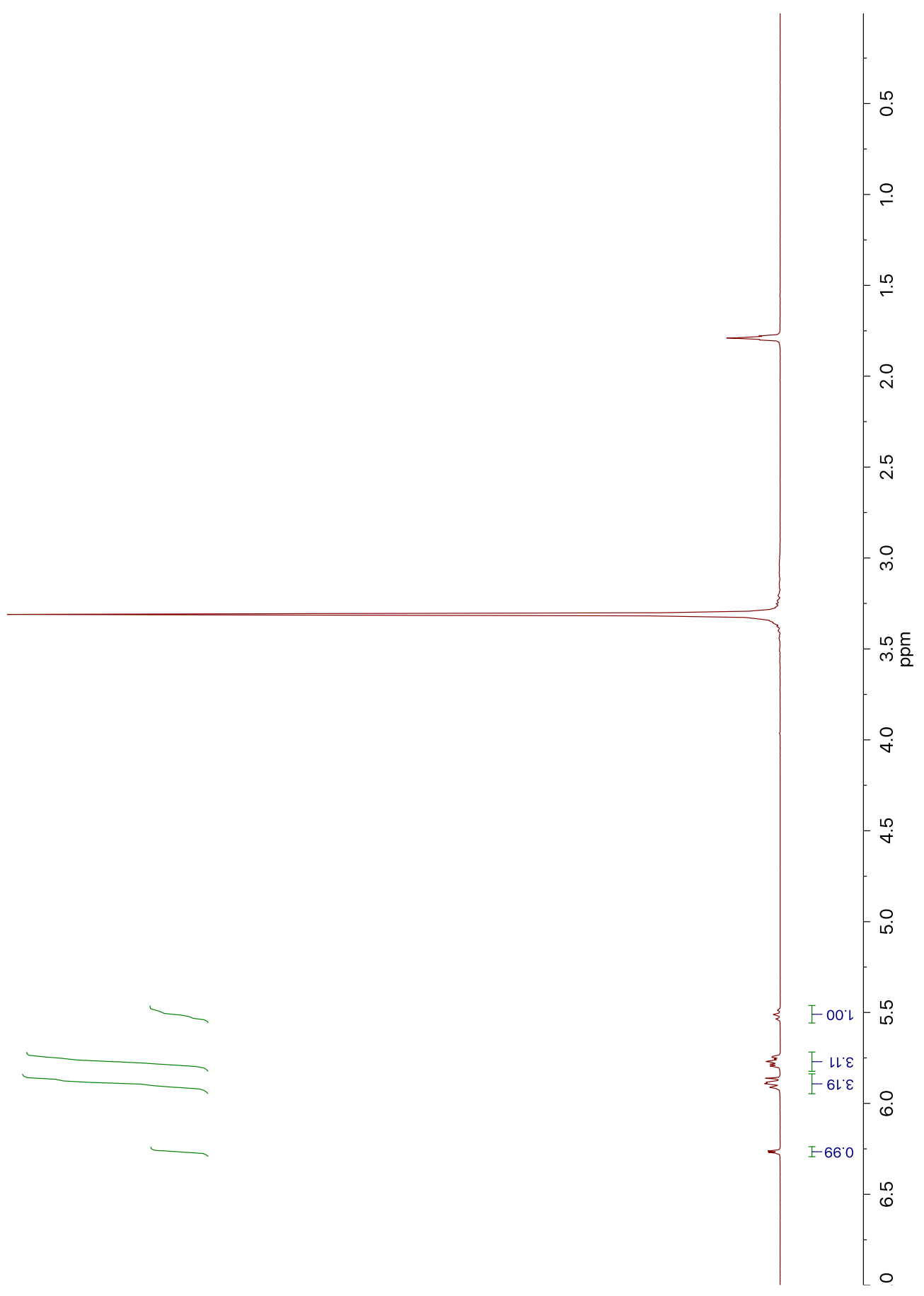

Figure 18. ${ }^{1} \mathrm{H}$ NMR $\left(\mathrm{CD}_{3} \mathrm{OD}, 400 \mathrm{MHz}\right)$ spectrum of di-CC. 


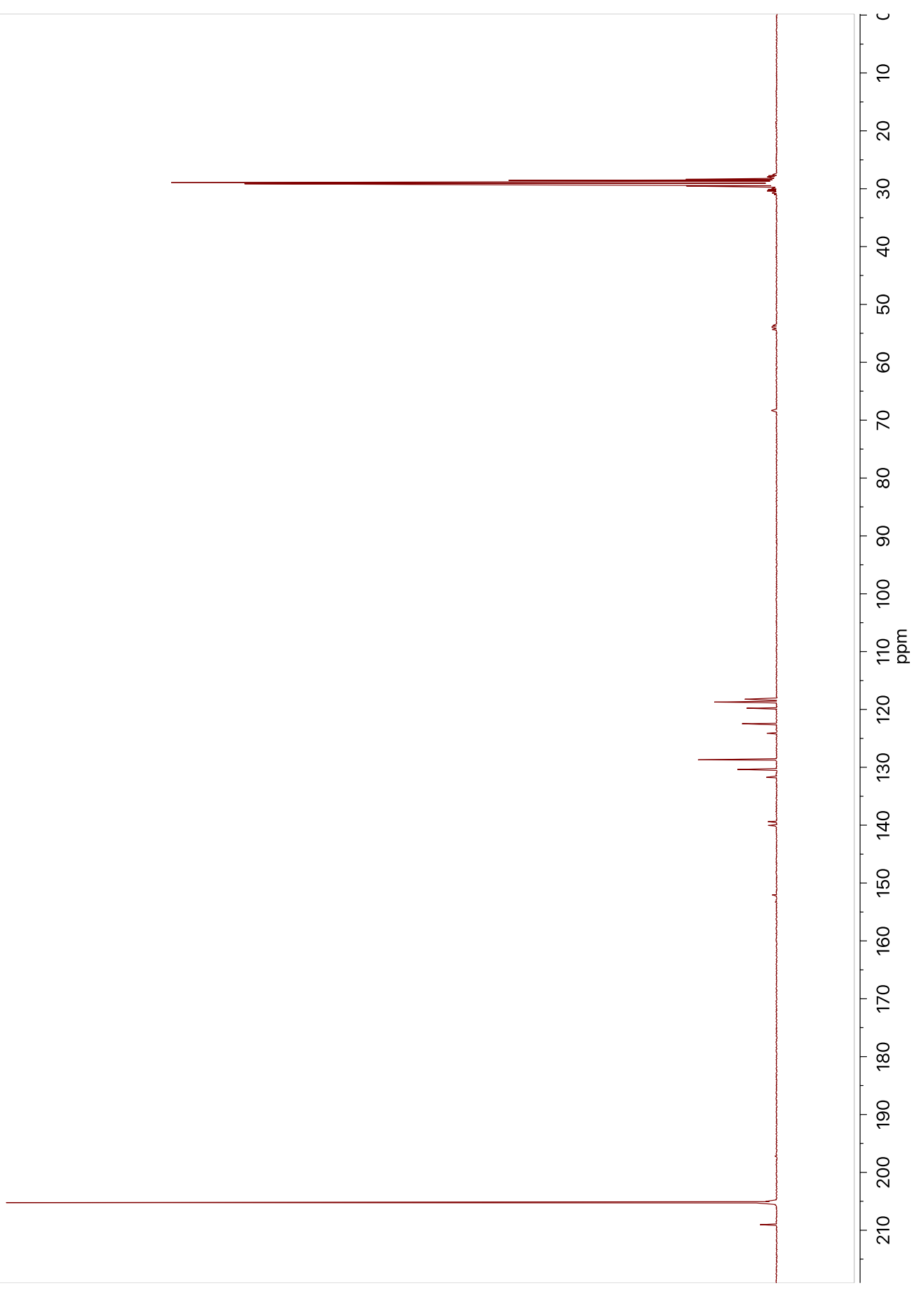

Figure 19. ${ }^{13} \mathrm{C}$ NMR $\left(\mathrm{CD}_{3} \mathrm{OD}, 100 \mathrm{MHz}\right)$ spectrum of di-CC. 


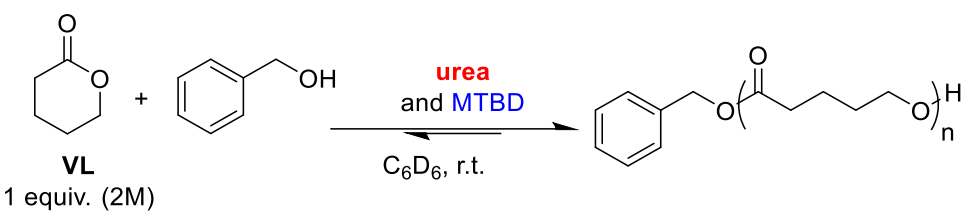

$\begin{array}{ccccccc}\text { entry } & \text { urea } & {[\mathrm{M}]_{\mathrm{o}} /[\mathrm{l}]_{\mathrm{o}}} & \text { time }(\mathrm{min}) & \text { conv. }(\%)^{\mathrm{a}} & M_{\mathrm{n}}^{\mathrm{b}} & M_{\mathrm{w}} / \mathrm{M}_{\mathrm{n}}^{\mathrm{b}} \\ 1 & \text { TCC } & 50 & 14 & 90 & 8500 & 1.08 \\ 2 & & 100 & 22 & 91 & 19900 & 1.05 \\ 3 & & 200 & 46 & 90 & 35900 & 1.0 \\ 4 & & 500 & 125 & 90 & 72900 & 1.02 \\ 5 & \text { di-CC } & 50 & 15 & 88 & 6000 & 1.04 \\ 6 & & 100 & 20 & 89 & 12000 & 1.04 \\ 7 & & 200 & 78 & 94 & 25000 & 1.03 \\ 8 & & 500 & 180 & 89 & 64000 & 1.06\end{array}$

Table 5. Chain Length Variation for the TCC or di-CC plus MTBD cocatalyzed ROP of VL. a. Conversion determined by ${ }^{1} \mathrm{H}$ NMR. b. $M_{\mathrm{n}}$ and $M_{\mathrm{w}}$ were obtained by GPC. 


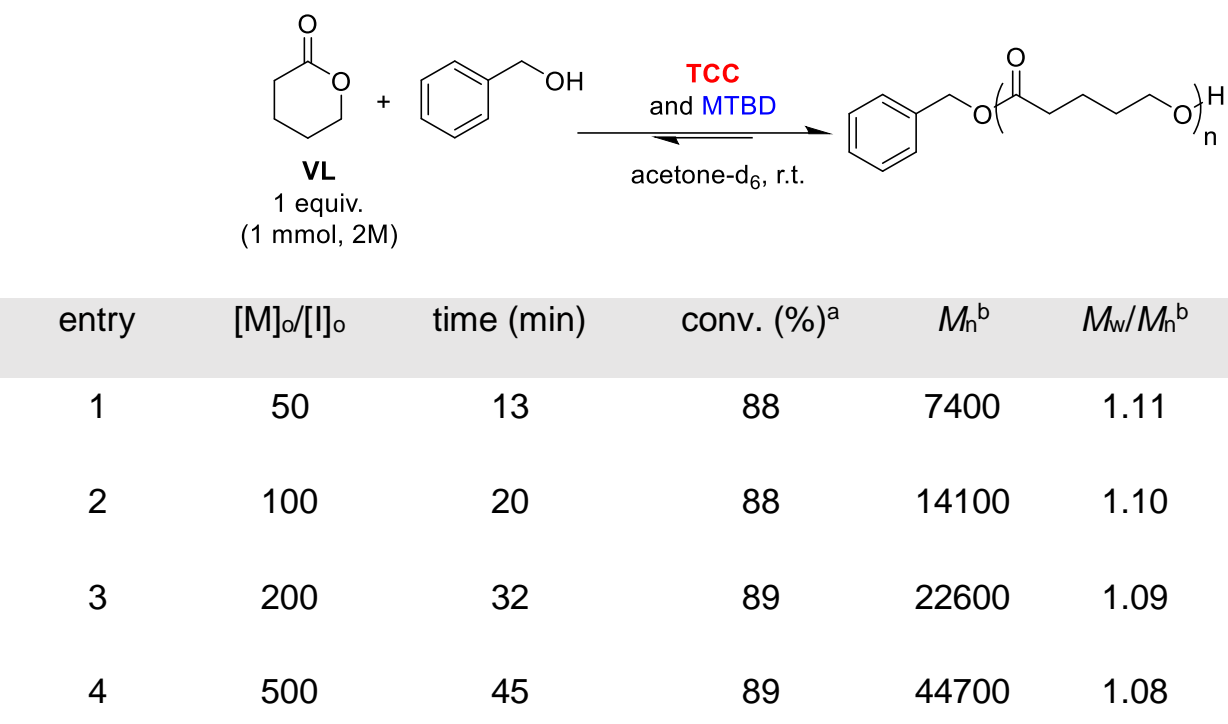

Table 6. Chain Length Variation for the TCC/MTBD cocatalyzed ROP of VL in acetone- $d_{6}$. a. Conversion determined by ${ }^{1} \mathrm{H}$ NMR. b. $M_{\mathrm{n}}$ and $M_{\mathrm{w}}$ were obtained by GPC. 


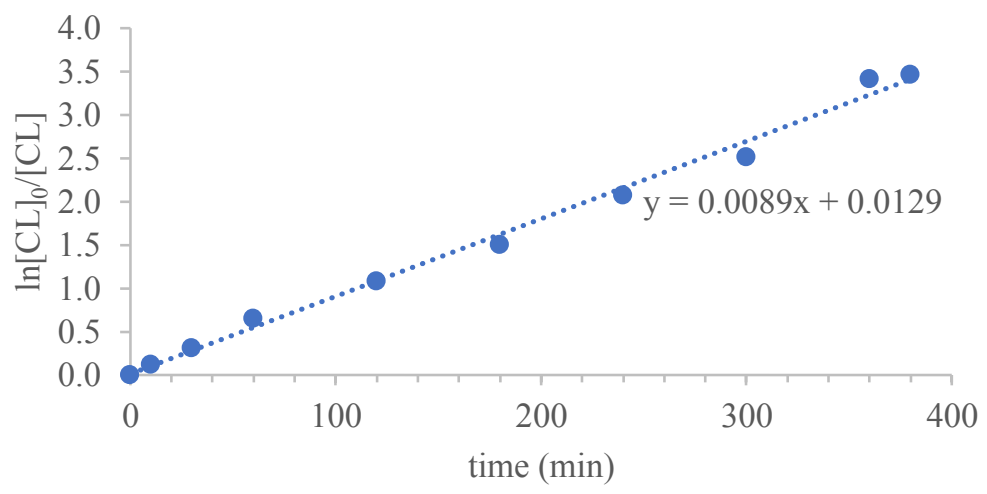

Figure 20. First order evolution of $\mathrm{CL}$ vs time for the TCC/MTBD catalyzed ring-opening polymerization of CL. Conditions: CL (2 M, $1 \mathrm{mmol})$, benzyl alcohol (1 $\mathrm{mol} \%, 0.01 \mathrm{mmol})$, TCC $(5 \mathrm{~mol} \%, 0.04 \mathrm{mmol})$, MTBD (5 mol\%, $0.04 \mathrm{mmol})$ in benzene- $d_{6}$. 


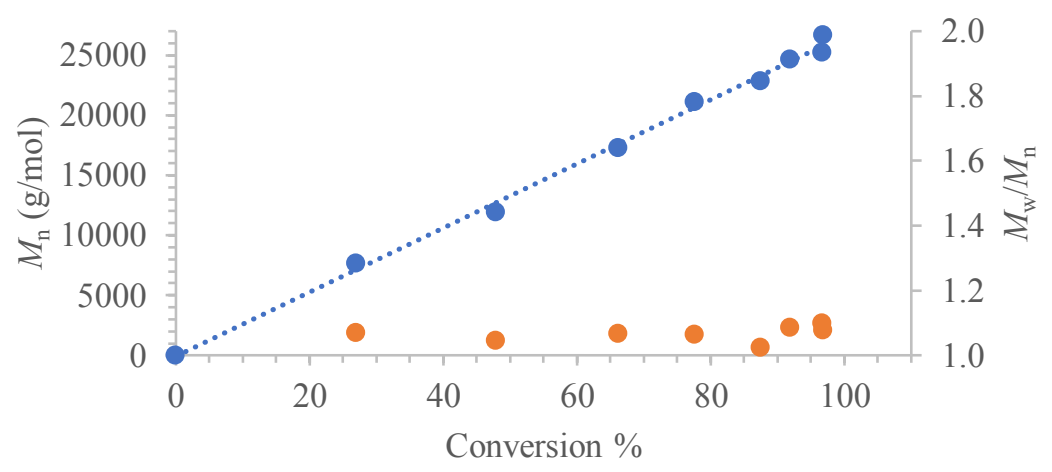

Figure 21. $M_{\mathrm{n}}$ (blue) and $M_{\mathrm{w}} / M_{\mathrm{n}}$ (orange) catalyzed ring-opening polymerization of CL. Conditions: CL (2 M, 1mmol), benzyl alcohol (1mol\%, $0.01 \mathrm{mmol})$, TCC (5mol\%, $0.04 \mathrm{mmol})$, MTBD (5 mol\%, $0.04 \mathrm{mmol})$ in benzene- $d_{6}$.

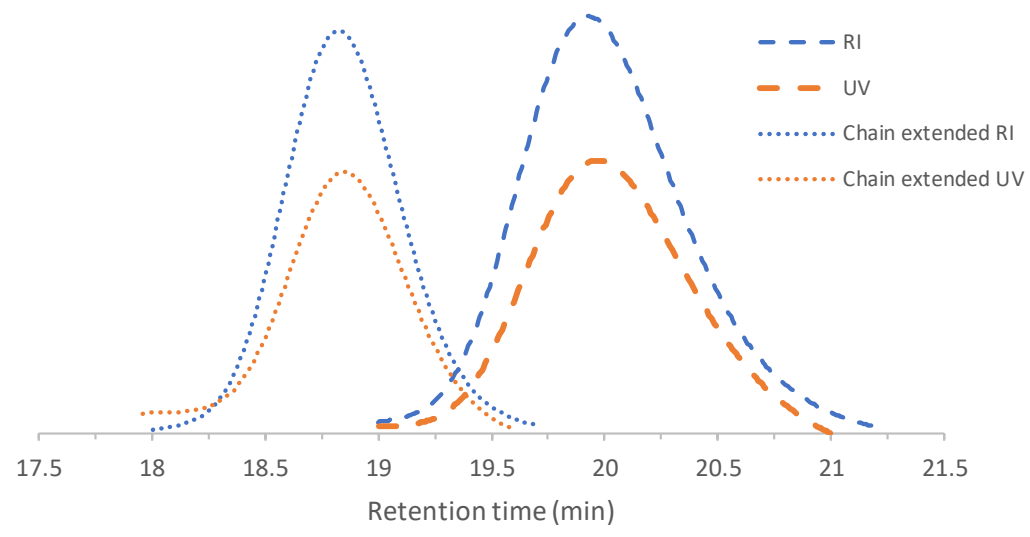

Figure 22. GPC traces of the polymers resulting from the chain extension experiment of VL. Conditions: VL ( $1 \mathrm{M}, 1 \mathrm{mmol}), 1$-pyrenebutanol $(2 \mathrm{~mol} \%, 0.02 \mathrm{mmol})$, TCC (5 mol\%, 0.05 $\mathrm{mmol})$, BEMP ( $5 \mathrm{~mol} \%, 0.05 \mathrm{mmol})$ in benzene- $d_{6}$, and subsequent chain extension by the addition of $\mathrm{VL}(1 \mathrm{mmol})$. 


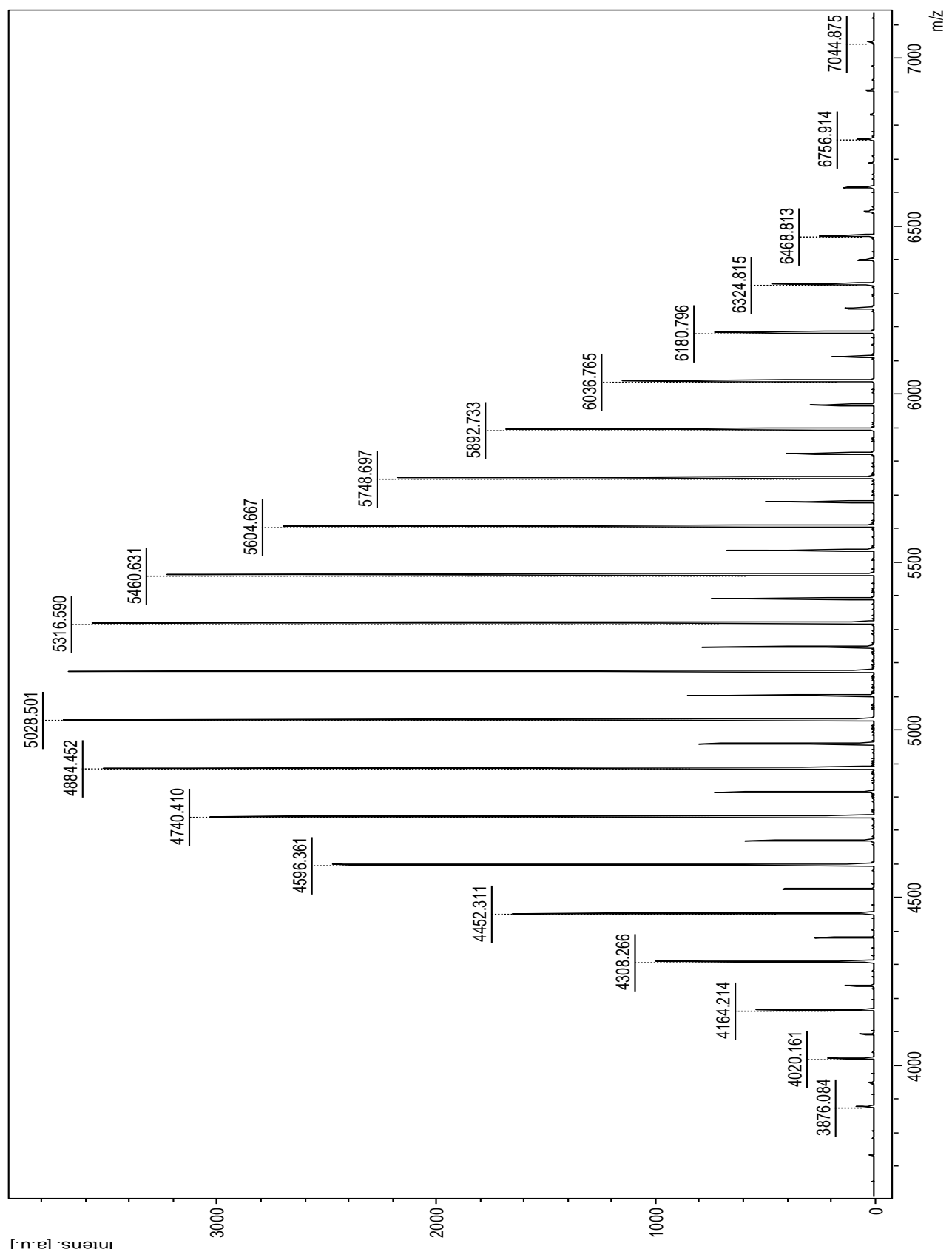

Figure 23. MALDI-TOF of the PLLA resulting from TCC/Me 6 TREN cocatalyzed ROP of Llactide. The major pattern (blue line) is due to whole repeat units $\mathrm{m} / \mathrm{z}=\left(\mathrm{Na}^{+}+\right.$benzyl alcohol $+n^{*} L A$ ) while the minor pattern (red line) is due to half repeat units generated by postpolymerization transesterification $\mathrm{m} / \mathrm{z}=\left(\mathrm{Na}^{+}+\right.$benzyl alcohol $\left.+(\mathrm{n}+1 / 2)^{*} \mathrm{LA}\right)$. All $\mathrm{m} / \mathrm{z}$ bear a benzyl alcohol initiator. 


\section{CHAPTER 4}

Prepared for publication in ACS Macro Letters.

Bis-thiourea mediated organocatalyzed ROP of a cyclic lactone

Timothy J. Bannin

Chemistry, University of Rhode Island, Kingston, RI USA 


\section{ABSTRACT}

Highly efficient, selective, and expeditious rates are qualities desired when developing organocatalysts that effect the ring-opening polymerization of cyclic esters. The polymerization of $\delta$-valerolactone is conducted employing several new bis-thiourea co-catalysts. These thiourea co-catalysts employ two active binding sites; successfully increasing the rate of polymerization. The MTBD/5CTU, MTBD/DBU, and MTBD/BEMP organocatalytic systems were tested and several kinetic observations were made. Calculating the $k_{i e}$ of the DBU/5CTU system led yielded a mechanistic insight. Several interesting trends were observed when

comparing the initial rates of polymerization. The bis-thiourea/base system remains a controlled, quick, and selective way to polymerize cyclic esters and carbonates. 
$\mathrm{H}$-bonding catalysts for ring-opening polymerizations (ROP) provide efficient and highly controllable (i.e. molecular weight and polydispersity) pathways to a myriad of polyesters and polycarbonates. ${ }^{1-6}$ The ability to control and tailor these systems by exploiting a "living" pathway is tenaciously sought after. The $\mathrm{H}$-bond mediated ROP of esters is traditionally thought to proceed via a "living" pathway where the monomer, typically a $\mathrm{H}$-bond donor (thiourea or urea), is activated simultaneously with the alcohol chain end, usually a $\mathrm{H}$-bond accepting base. ${ }^{7,8}$ Traditionally, metal catalyzed polymerizations (e.g. olefin metathesis) exhibit better control and rates of reaction than organocatalyzed ROP. Expanding the scope of organocatalytic systems available for application is a major focus of research. Compared to metal catalysts, organocatalysts appear to fall under one of two categories, highly selective or highly active. Our lab has recently disclosed organocatalyzed ROP conditions that can rival the rate and control of metal catalyzed ROP conditions. ${ }^{6,9-11}$ However, stereoselectivity is still a beast polymer chemists have yet to completely tame when conducting the organocatalyzed $\mathrm{H}$-bond mediated ROP. The development of $\mathrm{H}$-bonding catalyst systems to affect the organocatalyzed ROP of cyclic lactones and carbonates has been a cornerstone of research in our lab. The following is a comparison of several thiourea based catalysts developed to increase both the "livingness" and rate of reaction.

After employing traditional ROP organocatalysts towards the polymerization of several monomers, our lab sought to expand the library of ROP H-bond catalytic systems. These cocatalysts were fashioned after the thiourea/base mediated co-catalytic systems originally developed at IBM Almaden and Stanford university by Hedrick and Waymouth, respectively, Figure $1.1,8$ These catalytic systems employ $\mathrm{H}$-bond activation of the monomer and initiator to affect the ROP of certain cyclic monomers. The typical scope of monomers employed includes cyclic carbonates, lactones, and lactams. IBM employed both a single catalyst that mutually houses the $\mathrm{H}$-bond donating and accepting moieties and a system that splits the active sites into two molecules. Our lab has focused on the activity of the catalytic systems when independent of each other. 

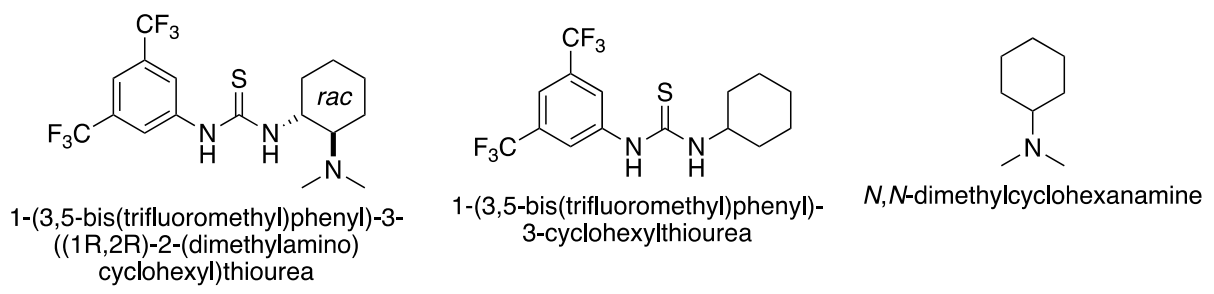
cyclohexyl)thiourea

Figure 1. Catalytic systems employed by Hedrick and Waymouth.

Specifically, we have employed bis-thioureas where the carbon chain linker varies in length, Figure 2 (nCTU). We believe this will not only increase the rate of polymerization but also affect the selectivity (i.e. proclivity towards monomer compared to polymer) and increase the scope of monomers that are capable of being polymerized by these systems.

Our first bis-thiourea co-catalyst, 1,1'-(propane-1,3-diyl)bis(3-(3,5bis(trifluoromethyl)phenyl)thiourea) (3CTU, Figure 2) was synthesized via an adopted method

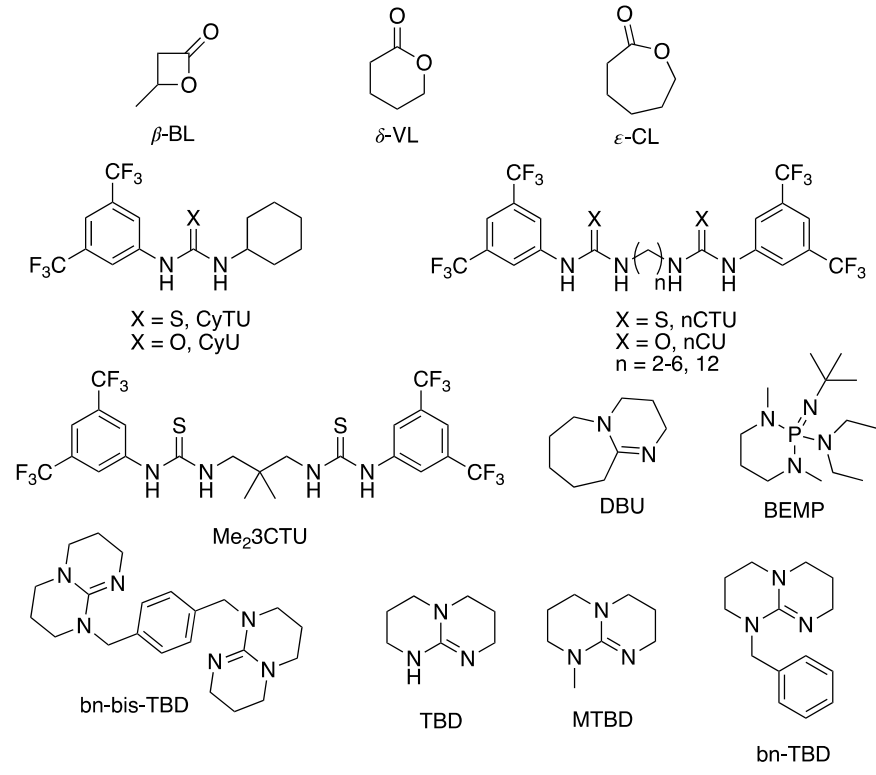

Figure 2. Structures and identifying abbreviations for compounds mentioned.

published by Bertucci et al and screened with several monomers, Table 1. Previously in our lab, we have shown when compared to the base/CyTU system, the base/3CTU system exhibited an accelerated rate of polymerization when applied to the organocatalyzed ROP of $I$ lactide. ${ }^{11}$ When initiated from benzyl alcohol $(2.0 \mathrm{~mol} \%)$ in benzene- $d_{6}$, the 3 CTU/MTBD $(2.5$ 
mol\% each) catalyzed ROP of VL (100 mg, $2 \mathrm{M}$ ) increased in rate compared to the CyTU/MTBD (2.5 and 5 mol\% each) catalyzed ROP of VL, Table 1 entries 2 and 4 . The new 3CTU/MTBD system proceeded with the first-order consumption of monomer, Figure 3. Success with 3CTU led our lab to develop several more thiourea based co-catalysts and evaluate them when applied to the organocatalyzed ROP of VL.

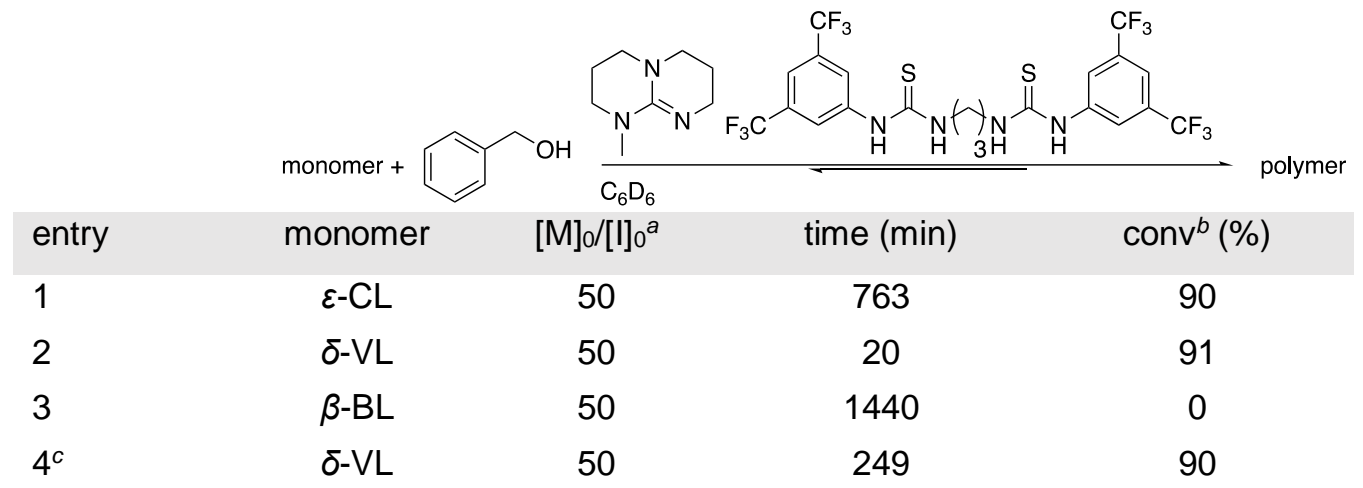

Table 1. MTBD/3CTU catalyzed ROP of CL, VL, and BL. Reaction conditions: monomer (100 $\mathrm{mg}, 2 \mathrm{M}$ each); initiated by benzyl alcohol ( $2.0 \mathrm{~mol} \%)$; catalyzed by MTBD/3CTU $(2.5 \mathrm{~mol} \%$ each); in benzene- $d_{6}$. ${ }^{a}$ Conversion tracked by ${ }^{1} \mathrm{HNMR} .{ }^{b}$ Calculated by GPC on the basis of polystyrene standards eluted by $\mathrm{CH}_{2} \mathrm{Cl}_{2}$. ${ }^{c} \mathrm{MTBD} / \mathrm{CyTU}(2.5 \mathrm{~mol} \%)$ for comparison.
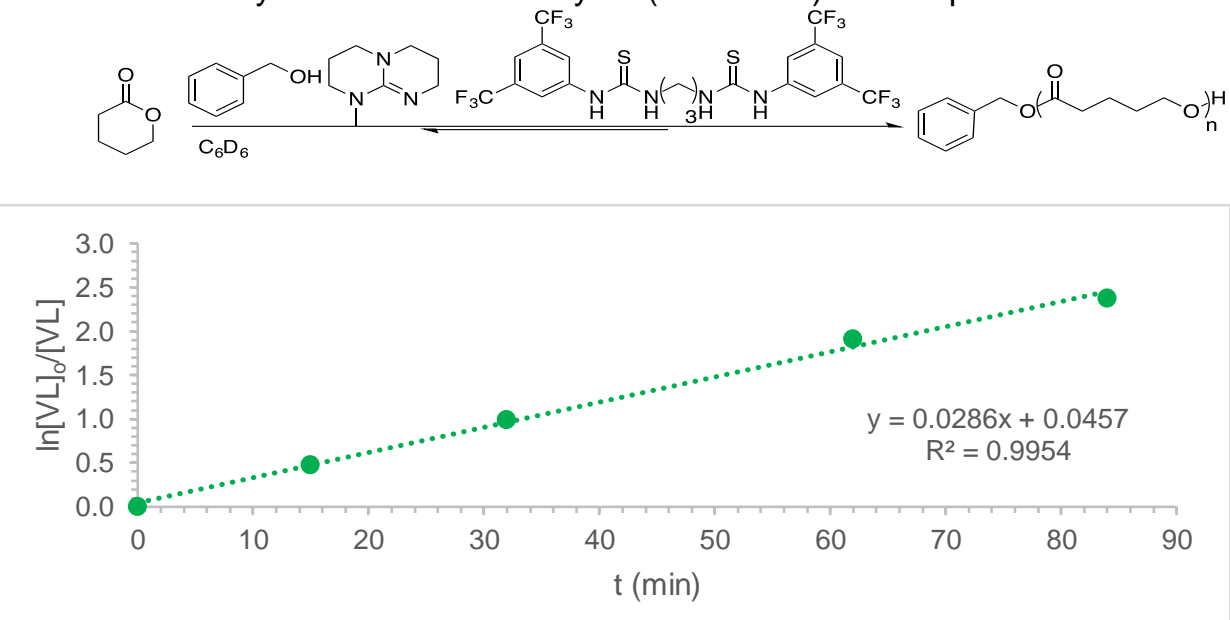

Figure 3. First-order evolution of [VL] vs. time for the 3CTU/MTBD catalyzed ROP of VL. Reaction conditions: VL (100 mg, $2 \mathrm{M})$, benzyl alcohol (2.00 mol \%), MTBD/3CTU (2.50 mol \% each), in benzene- $d_{6}$.

Variation of the carbon chain length between the two thiourea moieties led to varied reaction rates when applied to the polymerization of VL, Table 2 . When catalyzed by nCTU/MTBD (2.5 $\mathrm{mol} \%$ each), the polymerization of $\mathrm{VL}(100 \mathrm{mg}, 2 \mathrm{M})$ in benzene- $d_{6}$, initiated from benzyl alcohol 
$(2.0 \mathrm{~mol} \%)$ exhibited increased rate characteristics in all thiourea co-catalysts except 12CTU when compared to the CyTU/MTBD catalyzed polymerization of VL, Table 2. When the different catalyst systems were compared to each other, it was noticed that 12CTU/MTBD is slower than

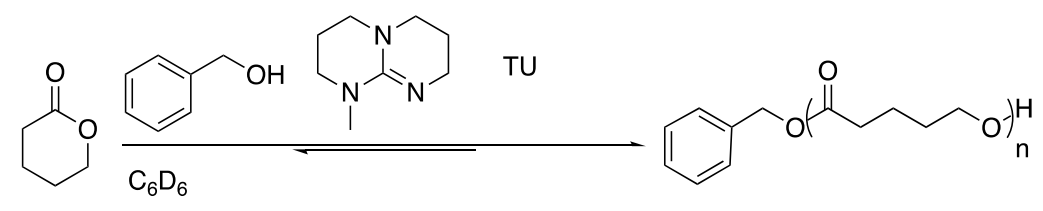

$\begin{array}{ccccccc}\text { entry } & \text { TU } & {[\mathrm{M}]_{\mathrm{o} /} /[\mathrm{l}]_{\circ}} & \text { time }(\mathrm{min}) & \text { conv }^{\mathrm{a}}(\%) & M_{\mathrm{n}}{ }^{b} & M_{\mathrm{w}} / \mathrm{Mn}^{b} \\ 1^{\mathrm{b}} & \text { CyTU } & 50 & 98 & 85 & 2400 & 1.11 \\ 2 & \text { CyTU } & 50 & 249 & 90 & 2500 & 1.10 \\ 3 & \text { 2CTU } & 50 & 58 & 86 & 4400 & 1.11 \\ 4 & 3 \mathrm{CTU} & 50 & 84 & 91 & 6100 & 1.10 \\ 5 & \text { 4CTU } & 50 & 36 & 90 & 7800 & 1.10 \\ 6 & 5 \mathrm{CTU} & 50 & 37 & 90 & 5900 & 1.10 \\ 7 & \text { 6CTU } & 50 & 58 & 89 & 6100 & 1.10 \\ 8 & \text { 12CTU } & 50 & 329 & 90 & 2000 & 1.11\end{array}$

Table 2. Variation of the thiourea co-catalyst in the MTBD/nCTU catalyzed ROP of VL. Reaction conditions: VL (100 mg, $2 \mathrm{M})$, benzyl alcohol (2.0 mol \%), MTBD/TU (2.5 mol \% each), benzene- $d_{6} .{ }^{a}$ Conversion tracked by ${ }^{1} \mathrm{HNMR} .{ }^{b}$ Calculated by GPC on the basis of polystyrene standards eluted by $\mathrm{CH}_{2} \mathrm{Cl}_{2}$. ${ }^{c} \mathrm{MTBD} / \mathrm{CyTU}$ (5.0 mol \%) for comparison.

CyTU/MTBD. The longer carbon chain connecting the dual active sites in the 12CTU appears to mimic a single moiety catalyst (CyTU). Conversely, the 2CTU and 6CTU appear to be closely related in rate, both reaching $87-90 \%$ completion in $\sim 58$ minutes. The two fastest thiourea cocatalysts tested were the 4CTU and 5CTU. In an attempt to increase the rate of polymerization, a thiourea with two methyl substituents attached to the center carbon of the linker between the TU moieties of 3CTU; Me 3 CTU (Figure 2), was synthesized. The addition of the two methyl groups in the carbon backbone was hypothesized to increase the rate of polymerization by taking advantage of the Thorpe-Ingold effect; opening up the active sites and causing them to interact with each other instead of adopting a more linear configuration. Ingold showed when two methyl substituents are added to a carbon, the groups attached to said carbon are in closer proximity when compared to the same, or similar, groups attached to a carbon bearing hydrogens, Figure $4 .{ }^{12}$ However, when tested, the Thorpe-Ingold TU was not soluble in 
benzene- $d_{6}$ and the ROP was extremely attenuated in acetone- $d_{6}$, Table 4 entry 4 . The ThorpeIngold effect appears to be counterproductive to the ROP. When applied to the TU catalyst, the
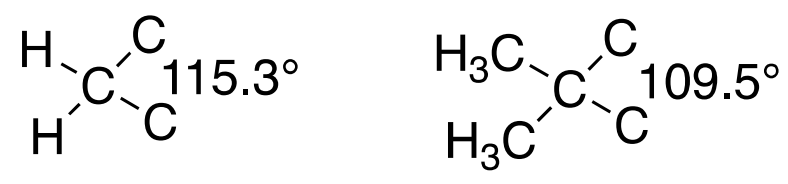

Figure 4. Angles determined by Ingold. ${ }^{12}$

Thorpe-Ingold effect may force the $\mathrm{Me}_{2} 3 \mathrm{CTU}$ to adopt a more rigid conformation thereby lending to the inability to naturally flex while in the transition state; severely hindering the activation of the monomer. Several other nCTU catalysts were tested in acetone- $d_{6}$ for comparison, Table 3. However, all nCTU's tested exhibited extremely attenuated rates of polymerization when compared to polymerizations preformed in benzene- $d_{6}$, Table 3 entries 1-3 and Table 2 entries 2,4 , and 6 . The slower rates are thought to be affected by the competitive hydrogen bonding of acetone with the active sites of the nCTU catalyst, of which typically activates the monomer. However, binding studies have proven difficult due to the lack of solubility of the nCTU catalysts. $\mathrm{H}$-bond donors 5CTU and 4CTU exhibit approximately the same rate of polymerization, and $5 \mathrm{CTU}$ was chosen for further analysis.

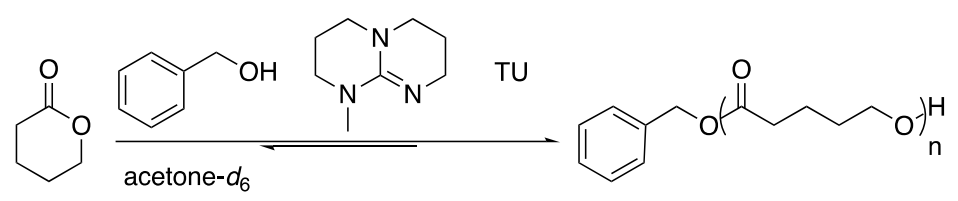

$\begin{array}{lccc}\text { entry } & \text { TU } & \text { time }(\mathrm{h}) & \text { conv. }^{a}(\%) \\ 1 & \text { CyTU } & 20.1 & 89 \\ 2 & \text { 3CTU } & 17.4 & 90 \\ 3 & \text { 5CTU } & 12.3 & 91 \\ 4 & \text { Me23CTU } & 16.2 & 54\end{array}$


Table 3. MTBD/nCTU catalyzed ROP of VL in acetone-d6. Reaction conditions: VL (2 M), benzyl alcohol $(2.0 \mathrm{~mol} \%)$ in acetone- $d_{6}$ catalyzed by MTBD/nCTU ( $2.5 \mathrm{~mol} \%$ each). a Conversion tracked by ${ }^{1} \mathrm{HNMR}$.

The reaction conditions for the $5 \mathrm{CTU} /$ base catalyzed ROP of VL were varied in an attempt to not only increase control and rate, but also in an attempt to learn more about the mechanistic properties. Several H-bond accepting bases were tested to see how the co-catalytic systems (nCTU/base) affect the organocatalyzed ROP of VL. Using TBD (Figure 2), a well-known transesterification catalyst employed in the ROP of cyclic lactones, ${ }^{7,8}$ as a basic scaffold for an $H$-bond accepting co-catalyst, several functionalized catalysts were synthesized and tested (bnTBD and bn-bis-TBD) with the ROP of VL,. The imine base, DBU, amidine bases, TBD, MTBD, bn-TBD, bn-bis-TBD, and phosphazine base, BEMP, (Figure 2) were all active co-catalysts in the ROP of VL. When the percent loading of base was varied, the rate of the polymerization became more attenuated as the base increased in bulkiness, except in the case of BEMP. The MTBD/nCTU and DBU/nCTU systems both exhibit narrow molecular weight distributions, a linear evolution of $M_{\mathrm{n}}$ versus conversion, and molecular weight controlled by adjusting the $[\mathrm{M}]_{\mathrm{o}} /[\mathrm{I}]_{\mathrm{o}}$ ratio (see Supplemental Information Figures S1-S2 and Tables S1-S2). 


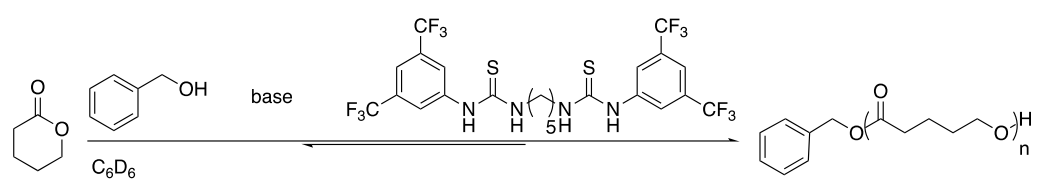

\begin{tabular}{l|ccc} 
entry & base & time $(\min )$ & conv. $^{a}(\%)$ \\
\hline 1 & MTBD & 37 & 90 \\
2 & DBU & 36 & 90 \\
3 & BEMP & 25 & 90 \\
4 & bn-TBD & 85 & 91 \\
5 & bn-bis-TBD & 100 & 90
\end{tabular}

Table 4. Base screen for the base/5CTU catalyzed ROP of VL. Reaction conditions: VL (2 M), benzyl alcohol $(2.0 \mathrm{~mol} \%)$ in benzene- $d_{6}$ catalyzed by base $/ 5 \mathrm{CTU}$ (2.5 mol \% each). Conversion tracked by ${ }^{1} \mathrm{HNMR}$.

Several kinetic studies were completed in an effort to learn something about the transition state; the binding of monomer and subsequent propagation. Several theories have arisen in our lab; the most prolific being a bis-thiourea used as a co-catalyst in the ROP of VL can theoretically bind two monomers as it contains two active sites to which monomer can bind. However, binding is most likely competitive between the solvent, monomer, and base. Binding studies were attempted, unfortunately solubility of the thiourea catalysts have made traditional NMR binding studies difficult. Our lab is currently addressing this problem.

Michaelis-Menten (M-M) kinetics test for inhibitory, competitive, or uncompetitive binding events, see Supplemental Information Figure S3. Our lab exploited M-M kinetics to test the MTBD/5CTU catalyst system for such events. The MTBD/5CTU system appeared to exhibit competitive inhibition; where the base competitively binds to the thiourea, see Supplemental Information Figures S4. However, an oddity manifest when graphing the $0^{\text {th }}, 1^{\text {st }}$, and $2^{\text {nd }}$ order plots of the consumption of monomer versus time.

Traditionally, it is thought these polymerizations proceed in a "living" manner; first-order consumption of monomer, controlled molecular weight by varying the $[\mathrm{M}]_{\mathrm{o}} /[]_{\mathrm{o}}$, narrow $M_{\mathrm{n}} / M_{\mathrm{w}}$, 
the linear conversion of $M_{n}$ with respect to time, and a living chain-end. However, with certain base/nCTU combinations we have noticed some catalyst pairs graphically exhibit the $2^{\text {nd }}$-order evolution of $1 /[\mathrm{VL}]$ versus time. Initially, this trend was noticed while comparing the $1^{\text {st }}$-order evolution of [VL] versus time for the MTBD/nCTU catalyzed ROP of VL. When the alkyl chain is short (CyTU, $n=2-4)$, the MTBD/nCTU (2.5 mol \% each) catalyzed polymerization of VL (100 mg, $1.00 \mathrm{mmol}, 2 \mathrm{M})$, initiated by benzyl alcohol $(2.0 \mathrm{~mol} \%)$ in benzene-d6 exhibit firstorder evolution of monomer, see Supporting Information Figures S5-S8. However, when the alkyl chain increased to $n=5,6$, and 12, holding all other conditions the same, the polymerization graphically exhibits a second-order evolution of monomer, see Supporting Information Figures S9-S11. MTBD was not the only base to graphically exhibit the secondorder consumption of monomer. Both DBU and BEMP also exhibited second-order consumption of monomer when paired with 4-6CTU and 2-6 and 12CTU, respectively, see Supporting Information Figures S12-21. Further testing is being conducted to unequivocally prove this trend; e.g. binding studies. While conducting these studies several kinetic trends were observed.

When conducting the polymerization of $\mathrm{VL}(100 \mathrm{mg}, 2 \mathrm{M})$, initiated by benzyl alcohol $(2.0 \mathrm{~mol}$ $\%)$ in the presence of base/nCTU (2.5 mol \% each), certain kinetic trends were discovered. The initial rate and comparative relative rate were extracted from the linear portion of the data, up to $\sim 50 \%$ conversion of the first-order evolution of [VL] vs time. Of the base/nCTU combinations studied the BEMP/nCTU catalyzed ROP of VL exhibited the fastest initial rates, except for the 6- and 12CTU co-catalysts, see Supplemental Information Tables S3. BEMP also exhibited the greatest difference in relative initial rates when compared. More work is being completed with the $\mathrm{BEMP/nCTU}$ system to further investigate this trend. When the base co-catalysts were compared, all three (MTBD, DBU, BEMP) exhibit similar trends; 1) As chain length increases from $n=2-5$ initial rate increases (except in the case of DBU) and 2) 4-, 5-, and 6CTU exhibit the fastest initial rates. In all three cases the CyTU/base catalyzed ROP of VL exhibited the slowest initial rate. The $\mathrm{k}_{\mathrm{e}}$ was also examined for the $5 \mathrm{CTU} / \mathrm{DBU}$ catalytic system, $\mathrm{k}_{\mathrm{ie}}=2.42$, see Supporting Information Figure S22; exhibiting a normal-primary isotope effect. An 
observation into the transition state can be glimpsed from this data; the O-H/O-D bond is broken in the rate determining step. This implies the alcoholic proton on the benzyl alcohol (initiator) can be readily exchanged with deuterium during polymerization theoretically impeding initiation, further implication of competitive inhibitory effects taking place. Further testing must be completed to identify the extent of inhibition, the species that acts as the inhibitor, and the $k_{i e}$ of the other base/nTCU catalytic systems.

In conclusion, it has been shown that a bis-thiourea co-catalyst not only increases the rate of polymerization, but also increases control of polymerization (narrow dispersion of molecular weights). When compared, 4- and 5CTU exhibited the fastest initial rates when applied to the base/nCTU catalyzed ROP of VL. The MTBD, DBU, and BEMP co-catalysts all graphically exhibited second-order evolution of monomer versus time when paired with certain nCTU cocatalysts. However, the reactions did exhibit other aspects of "living" polymerizations; e.g. a linear evolution of $M_{\mathrm{n}}$ versus conversion and controlled molecular weight by altering $[\mathrm{M}]_{\mathrm{o}} /[\mathrm{l}]_{\mathrm{o}}$. Overall, the scope of $\mathrm{H}$-bond organocatalytic systems has been increased. However, more work is being conducted to further prove/disprove the second-order evolution of monomer with respect to time and in order to gain more insight into the mechanistic characteristics of the base/nCTU catalytic system for the polymerization of cyclic lactones. 


\section{EXPERIMENTAL SECTION}

General Considerations. All chemicals were purchased from Fisher Scientific and used as received. $\delta$-Valerolactone and $\varepsilon$-caprolactone were distilled from calcium hydride $\left(\mathrm{CaH}_{2}\right)$ under vacuum (10 mTorr) and stored over 3Å molecular sieves. L-Lactide was recrystallized in toluene and dried under vacuum overnight. HPLC grade methylene chloride $\left(\mathrm{CH}_{2} \mathrm{Cl}_{2}\right)$ and tetrahydrofuran (THF) were purchased from Fisher Scientific and dried on an Innovative Technology solvent system featuring alumina columns. Chloroform, chloroform- $d$ (Cambridge Isotopes), and o-dichlorobenzene (o-DCB) were distilled from $\mathrm{CaH}_{2}$ under vacuum (10 mTorr), stored over $4 \AA$ molecular sieves, and passed through a plug of activated alumina just before use (except $o-\mathrm{DCB})$. Benzene and benzene- $d_{6}$ (Cambridge) were distilled from $\mathrm{CaH}_{2}$ under nitrogen atmosphere and stored over $4 \AA$ sieves. 1-[3,5-Bis(trifluoromethyl)phenyl]-3cyclohexylthiourea (CyTU) was made by following literature procedures. ${ }^{7}$ 2CTU, 3CTU, 4CTU, 5CTU, 6CTU, and 12CTU were all made by following a modified procedure. ${ }^{13}$ All reactions were performed in a glove box or by standard Schlenk techniques under $\mathrm{N}_{2}$ atmosphere. ${ }^{1} \mathrm{H}$ and ${ }^{13} \mathrm{C}$ NMR's were obtained utilizing a Bruker Avance III 300 and $400 \mathrm{MHz}$ instruments. Gel permeation chromatography (GPC) was performed in $\mathrm{CH}_{2} \mathrm{Cl}_{2}$ utilizing an Agilent Technologies 1260 Infinity fitted with three $5 \mu \mathrm{m}$ Agilent analytical columns connected in series with increasing

pore size $\left(10^{4}, 10^{3}, 50 \AA\right)$, an Agilent Infinity 1260 refractive index detector, and an Agilent Infinity $1260 \mathrm{UV} /$ Vis detector (250 $\mathrm{nm}$ and $300 \mathrm{~nm}$ ). $M_{\mathrm{n}}$ and $M_{\mathrm{w}} / M_{\mathrm{n}}$ were determined versus polystyrene standards $(500 \mathrm{~g} / \mathrm{mol}-3150 \mathrm{~kg} / \mathrm{mol}$, Polymer Laboratories). DFT calculations were run with Spartan '14 at the DFT B3LYP/6-31G* level of theory, gas phase. High pressure liquid chromatography mass spec (HPLCMS) masses were obtained utilizing a Thermo Electron (San Jose, CA, USA) LTQ Orbitrap XL mass spectrometer affixed with an electrospray ionization (ESI) interface, positive ions were produced and introduced into the instrument. Tune conditions for infusion experiments $(10 \mu \mathrm{L} / \mathrm{min}$ flow, sample concentration $<20 \mu \mathrm{g} / \mathrm{mL}$ in $50 / 50 \mathrm{v} / \mathrm{v}$ water/acetonitrile) were as follows: ionspray voltage, $5000 \mathrm{~V}$; capillary temperature, $275^{\circ} \mathrm{C}$; sheath gas ( $\mathrm{N}_{2}$, arbitrary units), 8; auxiliary gas ( $\mathrm{N}_{2}$, arbitrary units), 0 ; capillary voltage, $35 \mathrm{~V}$; and tube lens, $110 \mathrm{~V}$. Prior to analysis, instrument was calibrated for positive ions using Pierce 
LTQ ESI positive ion calibration solution (lot \#PC197784). Ion trap experiments used N2 as a collision gas with normalized collision energies (NCE) between 10-25 eV for multistage fragmentation. High-energy collision (HCD) experiments were performed with $\mathrm{He}$ as the collision gas with a NCE of $25 \mathrm{eV}$.

Example preparation of bisthioureas (2CTU, 3CTU, 4CTU, 5CTU, 6CTU, 12CTU). (Procedure adapted from Bertucci et al., Chem. Commun., 2013, 49, 2055-2057.) ${ }^{13}$ Under a $\mathrm{N}_{2}$ atmosphere a dried $50 \mathrm{~mL}$ Schlenk flask was charged with 3,5-bis(trifluoromethyl)phenyl isothiocyanate $(1.4 \mathrm{~mL}, 7.7 \mathrm{mmol}), \mathrm{CH}_{2} \mathrm{Cl}_{2}(20 \mathrm{~mL})$, and a stir bar. Next, ethylenediamine $(0.26$ $\mathrm{mL}, 3.9 \mathrm{mmol}$ ) was added drop wise via syringe over a 2-3 minute period and left to stir for 324 hours; a white solid will precipitate out of solution. After the reaction was completed, the solvent was removed by vacuum filtration (4CTU, 5CTU, 6CTU, 12CTU) or in vacuo to yield a white solid. The solid was purified by either rinsing the white solid with cold $\mathrm{CH}_{2} \mathrm{Cl}_{2}$ while filtration was taking place or via silica gel column chromatography (97:3 $\mathrm{CH}_{2} \mathrm{Cl}_{2}$ :Methanol) and dried in a vacuum oven to remove solvent and residual water which yielded a white solid in all cases.

2CTU and 3CTU previously reported in Bertucci et al., Chem. Commun., 2013, 49, 2055-2057.

\section{1,1'-(butane-1,4-diyl)bis(trifluoromethyl)phenyl)thiourea) (4CTU)}

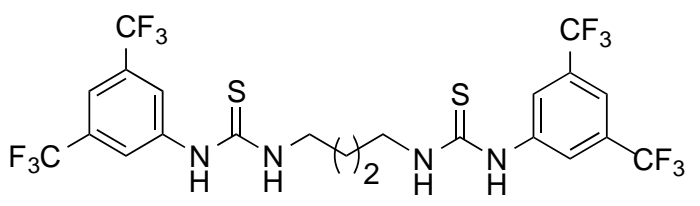

${ }^{1} \mathrm{H}$ NMR (Acetone- $\left.d_{6}, 300 \mathrm{MHz}\right): \delta=9.34(2 \mathrm{H}, \mathrm{s}), 8.30(4 \mathrm{H}, \mathrm{s}), 7.77(2 \mathrm{H}, \mathrm{s}), 7.70(2 \mathrm{H}, \mathrm{s}), 3.70$ $(4 \mathrm{H}, \mathrm{d}), 1.76(4 \mathrm{H}, \mathrm{m}) .{ }^{13} \mathrm{C}$ NMR (Acetone- $\left.d_{6}, 75 \mathrm{MHz}\right): \delta=182.31,142.88,131.95\left(\mathrm{q}, \mathrm{J}_{\mathrm{CF}}=33\right.$ $\mathrm{Hz}), 124.37\left(\mathrm{q}, \mathrm{J}_{\mathrm{CF}}=270 \mathrm{~Hz}\right), 123.48,117.49,44.73,26.86$. LCMS $(\mathrm{ESI}+)$ calcd for $\mathrm{C}_{22} \mathrm{H}_{19} \mathrm{~F}_{12} \mathrm{~N}_{4} \mathrm{~S}_{2}: 631.08$, found: $631.0825(\mathrm{M}+\mathrm{H})$. 
1,1'-(pentane-1,5-diyl)bis(trifluoromethyl)phenyl)thiourea) (5CTU)

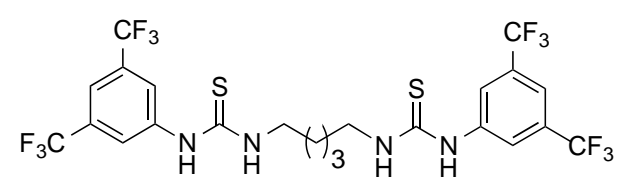

${ }^{1} \mathrm{H}$ NMR (Acetone- $d_{6}, 300 \mathrm{MHz}$ ): $\delta=9.34(\mathrm{~s}, 2 \mathrm{H}), 8.30(\mathrm{~s}, 4 \mathrm{H}), 7.77(\mathrm{~s}, 2 \mathrm{H}), 7.68(\mathrm{~s}, 2 \mathrm{H}), 3.65$ $(\mathrm{m}, 4 \mathrm{H}), 1.73(\mathrm{~m}, 4 \mathrm{H}), 1.48(\mathrm{~m}, 2 \mathrm{H}) .{ }^{13} \mathrm{C}$ NMR (Acetone- $\left.d_{6}, 75 \mathrm{MHz}\right): \delta=182.04,142.81,131.90$ $\left(q, J_{C F}=33 \mathrm{~Hz}\right), 124.33\left(q, J_{C F}=270 \mathrm{~Hz}\right), 123.21,117.33,44.89,29.07,24.90$. LCMS (ESI+) calcd for $\mathrm{C}_{23} \mathrm{H}_{21} \mathrm{~F}_{12} \mathrm{~N}_{4} \mathrm{~S}_{2}:$ : 645.09, found: $645.1016(\mathrm{M}+\mathrm{H})$.

\section{1,1'-(hexane-1,6-diyl)bis(trifluoromethyl)phenyl)thiourea) (6CTU)}

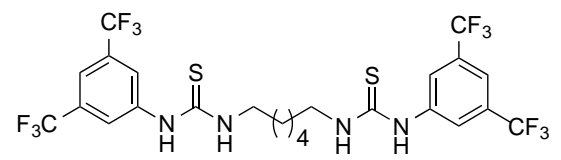

${ }^{1} \mathrm{H}$ NMR (Acetone- $d_{6}, 300 \mathrm{MHz}$ ): $\delta=9.29(\mathrm{~s}, 2 \mathrm{H}), 8.30(\mathrm{~s}, 4 \mathrm{H}), 7.73(\mathrm{~s}, 2 \mathrm{H}), 7.69(\mathrm{~s}, 2 \mathrm{H}), 3.64$ $(\mathrm{m}, 4 \mathrm{H}), 1.70(\mathrm{~m}, 4 \mathrm{H}), 1.45(\mathrm{~m}, 4 \mathrm{H}) .{ }^{13} \mathrm{C}$ NMR (Acetone- $\left.d_{6}, 75 \mathrm{MHz}\right): \delta=182.18,142.90,131.92$ $\left(q, J_{C F}=33 \mathrm{~Hz}\right), 124.36\left(q, J_{C F}=270 \mathrm{~Hz}\right), 123.35,117.38,45.07,27.31 . \quad$ LCMS (ESI+) calcd for $\mathrm{C}_{24} \mathrm{H}_{23} \mathrm{~F}_{12} \mathrm{~N}_{4} \mathrm{~S}_{2}: 659.11$, found: $659.1148(\mathrm{M}+\mathrm{H})$.

\section{1,1'-(dodecane-1,12-diyl)bis(trifluoromethyl)phenyl)thiourea) (12CTU)}

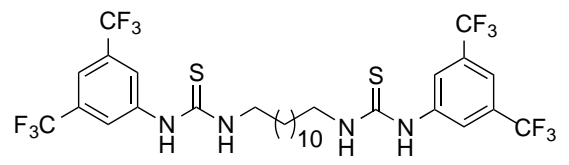

${ }^{1} \mathrm{H}$ NMR (Acetone- $d_{6}, 300 \mathrm{MHz}$ ): $\delta=9.28(\mathrm{~s}, 2 \mathrm{H}), 8.30(\mathrm{~s}, 4 \mathrm{H}), 7.72(\mathrm{~s}, 2 \mathrm{H}), 7.69(\mathrm{~s}, 2 \mathrm{H}), 3.63$ $(\mathrm{m}, 4 \mathrm{H}), 1.66(\mathrm{~m}, 4 \mathrm{H}), 1.36(\mathrm{~m}, 18 \mathrm{H}) .{ }^{13} \mathrm{C}$ NMR (Acetone- $\left.d_{6}, 75 \mathrm{MHz}\right): \delta=182.12,142.90$, $131.92\left(q, J_{C F}=33 \mathrm{~Hz}\right), 124.36\left(q, J_{C F}=270 \mathrm{~Hz}\right), 123.29,117.34,45.19,30.29,30.06,29.41$, 27.65. LCMS (ESI+) calcd for $\mathrm{C}_{30} \mathrm{H}_{35} \mathrm{~F}_{12} \mathrm{~N}_{4} \mathrm{~S}_{2}: 743.20$, found: $743.2086(\mathrm{M}+\mathrm{H})$.

Preparation of trisTU. Under a $\mathrm{N}_{2}$ atmosphere a dried $100 \mathrm{~mL}$ Schlenk flask was charged with 3,5-bis(trifluoromethyl)phenyl isothiocyanate (3.9 mL, $21.2 \mathrm{mmol})$, THF (50 mL), and a stir bar. Next, 2,2',2"-triaminotriethylamine $(1.05 \mathrm{~mL}, 6.8 \mathrm{mmol})$ was added drop wise via syringe over a 
2-3 minute period and left to stir overnight. After the reaction was completed, the solvent was removed in vacuo to yield a white solid. The product was purified via silica gel column chromatography (100\% ethyl acetate), rinsed with $\mathrm{CH}_{2} \mathrm{Cl}_{2}$, solvent removed in vacuo, and dried in a vacuum oven to remove residual water; which yielded a white solid. ${ }^{9}$

Example Polymerization of VL with MTBD and bisTU. VL (100 mg, $1.00 \mathrm{mmol}, 2 \mathrm{M})$ was mixed with one of the bisTU $(0.025 \mathrm{mmol})$ and dissolved in $90 \%$ of the total 0 -DCB $(449 \mu \mathrm{L})$ used in the reaction and added to a solution of benzyl alcohol (bnOH, $2.08 \mu \mathrm{L}, 0.020 \mathrm{mmol})$ and MTBD (3.59 $\mu \mathrm{L}, 0.025)$ made with the remaining o-DCB. Reaction was left to stir for $10 \mathrm{~min}$, quenched with benzoic acid $(7.0 \mathrm{mg})$, and solvent removed in vacuo to yield a white film. Conversion determined by NMR.

Example Michaelis-Menten Kinetics Study of the Polymerization of VL with MTBD (10 $\mathrm{mM}$ ) and 5CTU (5 mM). Four stock solutions were made; one each for VL (benzene- $d_{6}$ ), benzyl alcohol (benzene- $d_{6}$ ), MTBD (benzene- $d_{6}$ ), and 5CTU (solvated in $100 \mathrm{mg} \mathrm{VL}$ ), by weight. Enough stock solution was made to track 3 sets of 6 reactions; tracking one concentration of MTBD (10 mM, $100 \mathrm{mM}$, and $500 \mathrm{mM})$ at varying concentrations of VL $(6 \mathrm{M}, 4 \mathrm{M}, 2 \mathrm{M}, 1 \mathrm{M}, 0.5$ $\mathrm{M}$, and $0.25 \mathrm{M}$ ), holding the concentration of initiator (benzyl alcohol, $5 \mathrm{mM}, 1 \mathrm{~mol} \%$ ) and $5 \mathrm{CTU}$ $(5 \mathrm{mM}, 1 \mathrm{~mol} \%)$ constant in all reactions. The volume of all components together was held constant at $499.40 \mu \mathrm{L}$. All aliquots were measured by auto pipette. Twelve $7 \mathrm{~mL}$ scintillation vials were charged with stir bars. Then, six vials received the calculated volume of stock solution for MTBD (10 mM) and benzyl alcohol $(5 \mathrm{mM})$. Next, the remaining six vials received the calculated volume of stock solution for $5 \mathrm{CTU}(5 \mathrm{mM})$ and VL $(6 \mathrm{M}, 4 \mathrm{M}, 2 \mathrm{M}, 1 \mathrm{M}, 0.5 \mathrm{M}$, and $0.25 \mathrm{M})$. Then, the remaining amount of benzene- $d_{6}$ was weighed into the vial containing the MTBD/benzyl alcohol solution. Next, the MTBD/benzyl alcohol solution was pipetted into the vials containing the VL/5CTU solution. After these two solutions were mixed, each vial was pipetted into an NMR tube, capped, and tracked conversion overnight by ${ }^{1} \mathrm{HNMR}$ (acquiring spectra every 30 min for each sample for 12 hours). 


\section{LIST OF REFERENCES}

(1) Dove, A. P.; Pratt, R. C.; Lohmeijer, B. G. G.; Waymouth, R. M.; Hedrick, J. L. ThioureaBased Bifunctional Organocatalysis: Supramolecular Recognition for Living Polymerization. J. Am. Chem. Soc. 2005, 127 (40), 13798-13799.

(2) Kamber, N. E.; Jeong, W.; Waymouth, R. M.; Pratt, R. C.; Lohmeijer, B. G. G.; Hedrick, J. L. Organocatalytic Ring-Opening Polymerization. Chem. Rev. 2007, 107 (12), 58135840.

(3) Kiesewetter, M. K.; Shin, E. J.; Hedrick, J. L.; Waymouth, R. M. Organocatalysis: Opportunities and Challenges for Polymer Synthesis. Macromolecules 2010, 43 (5), 2093-2107.

(4) Bannin, T. J.; Kiesewetter, M. K. Poly(Thioester) by Organocatalytic Ring-Opening Polymerization. Macromolecules 2015, 48 (16), 5481-5486.

(5) Sanders, D. P.; Fukushima, K.; Coady, D. J.; Nelson, A.; Fujiwara, M.; Yasumoto, M.; Hedrick, J. L. A Simple and Efficient Synthesis of Functionalized Cyclic Carbonate Monomers Using a Versatile Pentafluorophenyl Ester Intermediate. J. Am. Chem. Soc. 2010, 132 (42), 14724-14726.

(6) Kazakov, O. I.; Datta, P. P.; Isajani, M.; Kiesewetter, E. T.; Kiesewetter, M. K. Cooperative Hydrogen-Bond Pairing in Organocatalytic Ring-Opening Polymerization. Macromolecules 2014, 47 (21), 7463-7468.

(7) Lohmeijer, B. G. G.; Pratt, R. C.; Leibfarth, F.; Logan, J. W.; Long, D. A.; Dove, A. P.; Nederberg, F.; Choi, J.; Wade, C.; Waymouth, R. M.; et al. Guanidine and Amidine Organocatalysts for Ring-Opening Polymerization of Cyclic Esters. Macromolecules 2006, 39 (25), 8574-8583.

(8) Pratt, R. C.; Lohmeijer, B. G. G.; Long, D. A.; Lundberg, P. N. P.; Dove, A. P.; Li, H.; Wade, C. G.; Waymouth, R. M.; Hedrick, J. L. Exploration, Optimization, and Application of Supramolecular Thiourea-Amine Catalysts for the Synthesis of Lactide (Co)Polymers. Macromolecules 2006, 39 (23), 7863-7871. 
(9) Fastnacht, K. V.; Spink, S. S.; Dharmaratne, N. U.; Pothupitiya, J. U.; Datta, P. P.; Kiesewetter, E. T.; Kiesewetter, M. K. Bis- and Tris-Urea H-Bond Donors for RingOpening Polymerization: Unprecedented Activity and Control from an Organocatalyst. ACS Macro Lett. 2016, 5 (8), 982-986.

(10) Dharmaratne, N. U.; Pothupitiya, J. U.; Bannin, T. J.; Kazakov, O. I.; Kiesewetter, M. K. Triclocarban: Commercial Antibacterial and Highly Effective H-Bond Donating Catalyst for Ring-Opening Polymerization. ACS Macro Lett. 2017, 6 (4), 421-425.

(11) Spink, S. S.; Kazakov, O. I.; Kiesewetter, E. T.; Kiesewetter, M. K. Rate Accelerated Organocatalytic Ring-Opening Polymerization of I-Lactide via the Application of a Bis(Thiourea) H-Bond Donating Cocatalyst. Macromolecules 2015, 48 (17), 61276131.

(12) Ingold, C. K. XL.-The Conditions Underlying the Formation of Unsaturated and of Cyclic Compounds from Halogenated Open-Chain Derivatives. Part I. Products Derived from a-Halogenated Glutaric Acids. J. Chem. Soc., Trans. 1921, 119 (0), 305-329.

(13) Bertucci, M. A.; Lee, S. J.; Gagné, M. R. Thiourea-Catalyzed Aminolysis of N-Acyl Homoserine Lactones. Chem. Commun. 2013, 49 (20), 2055-2057. 
SUPPORTING INFORMATION: Bis-thiourea mediated organocatalyzed ROP of a cyclic lactone
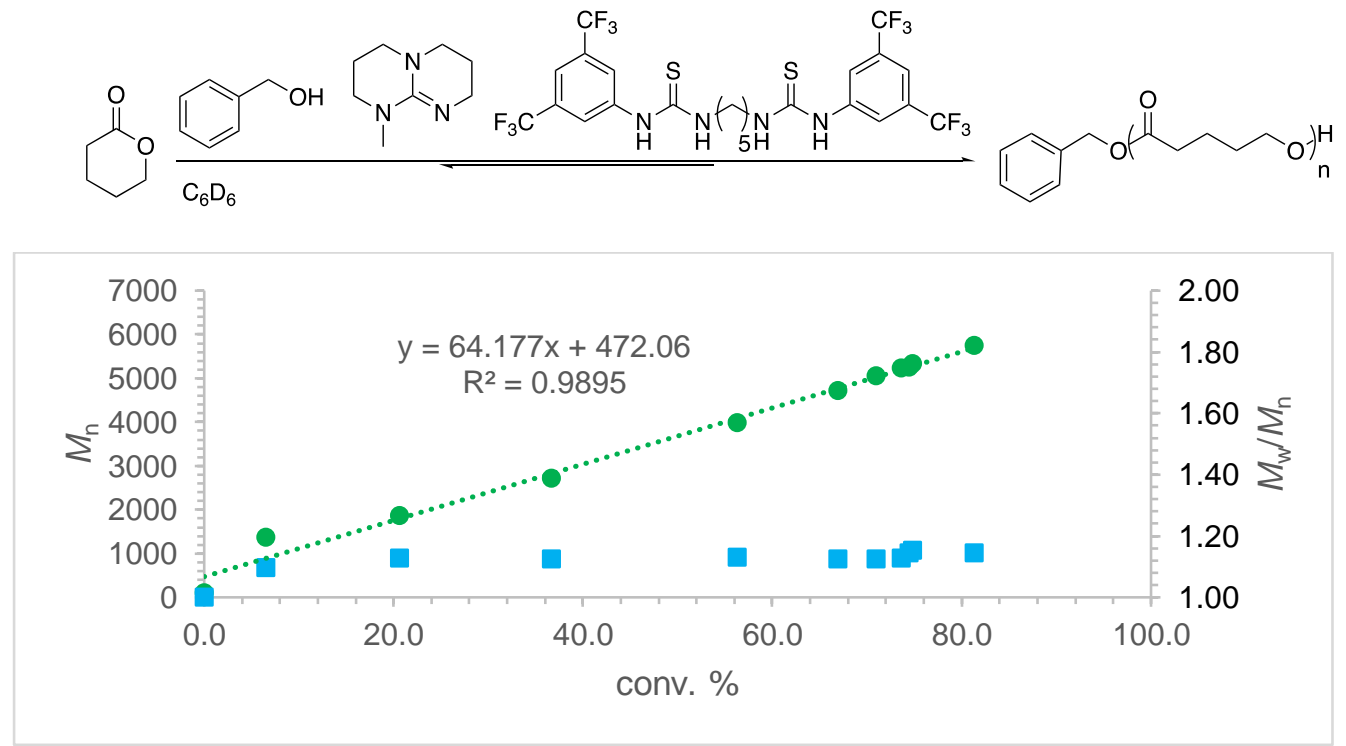

Figure S1. Linear conversion of $M_{\mathrm{n}}$ versus percent conversion (circles) and $M_{\mathrm{w}} / M_{\mathrm{n}}$ versus conversion (squares) for the MTBD/5CTU catalyzed ROP of VL. Reaction conditions: VL (2 M), benzyl alcohol $(2.0 \mathrm{~mol} \%)$ in benzene- $d_{6}$ catalyzed by MTBD/5CTU ( $2.5 \mathrm{~mol} \%$ each). Conversion tracked by NMR. 


\begin{tabular}{|c|c|c|c|c|c|}
\hline entry & {$[\mathrm{M}]_{\mathrm{o}} /[\mathrm{l}]_{\mathrm{o}}$} & time $(\min )$ & $\operatorname{conv}^{a}(\%)$ & $M_{n}^{b}$ & $M_{\mathrm{w}} / M_{\mathrm{n}}^{b}$ \\
\hline 1 & 50 & 10 & 92 & 5,100 & 1.10 \\
\hline 2 & 100 & 20 & 94 & 9,900 & 1.09 \\
\hline 3 & 200 & 30 & 94 & 16,000 & 1.09 \\
\hline
\end{tabular}

Table S1. $[\mathrm{M}]_{\mathrm{o}} /[\mathrm{l}]_{\mathrm{o}}$ screen for the MTBD/5CTU catalyzed ROP of VL. Reaction conditions: VL (2 M), benzyl alcohol $(0.5,1.0,2.0 \mathrm{~mol} \%)$ in benzene- $d_{6}$ catalyzed by MTBD/5CTU $(2.5 \mathrm{~mol} \%$ each). ${ }^{a}$ Conversion tracked by ${ }^{1} \mathrm{HNMR} .{ }^{b}$ Calculated by GPC on the basis of polystyrene standards eluted by $\mathrm{CH}_{2} \mathrm{Cl}_{2}$.

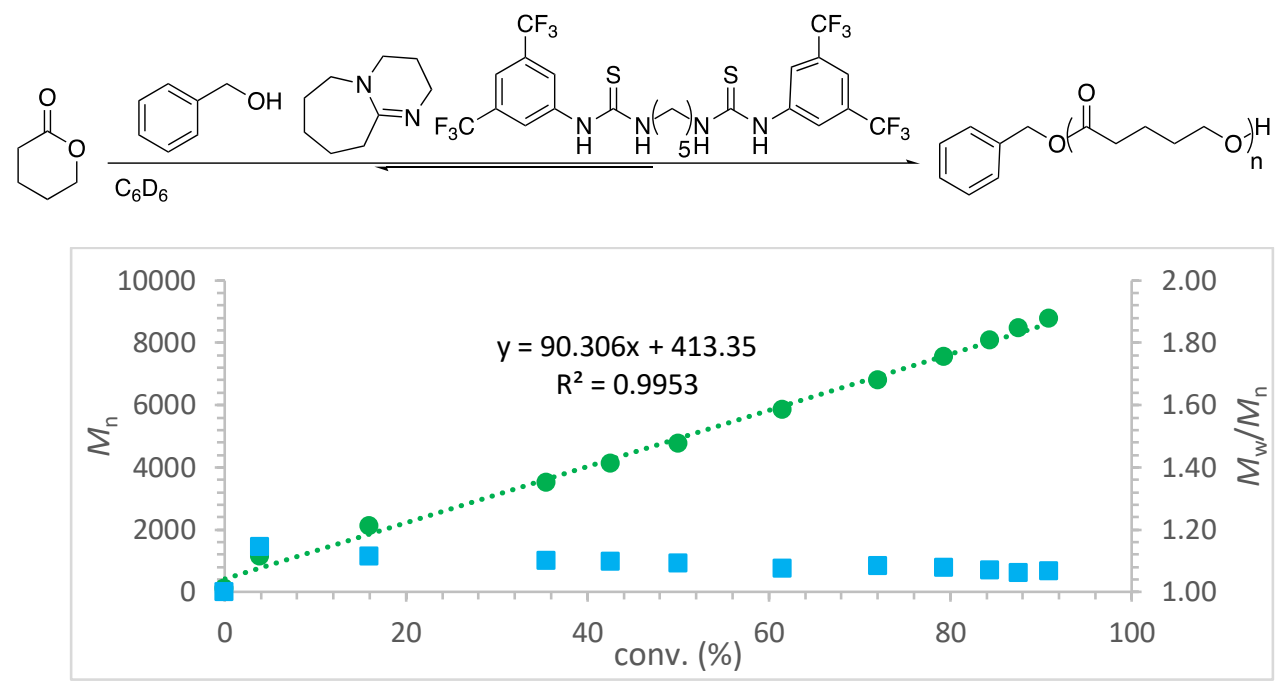

Figure S2. Linear conversion of $M_{\mathrm{n}}$ versus percent conversion (circles) and $M_{\mathrm{w}} / M_{\mathrm{n}}$ versus percent conversion (squares) of the DBU/5CTU catalyzed ROP of VL. Reaction conditions: VL $(2 \mathrm{M})$, benzyl alcohol $(2.0 \mathrm{~mol} \%)$ in benzene- $d_{6}$ catalyzed by DBU/5CTU $(2.5 \mathrm{~mol} \%$ each). Conversion tracked by NMR. $M_{\mathrm{n}}$ and $M_{\mathrm{w}} / M_{\mathrm{n}}$ calculated by GPC on the basis of polystyrene standards eluted by $\mathrm{CH}_{2} \mathrm{Cl}_{2}$. 


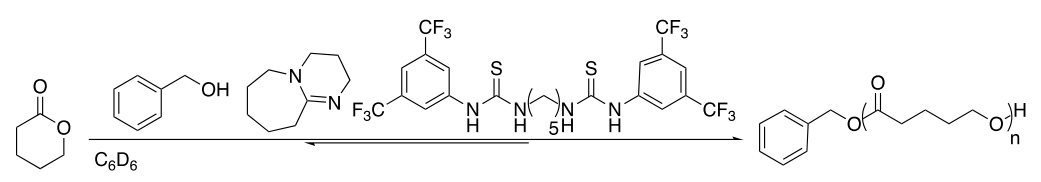

$\begin{array}{lcccc}\text { entry } & {[\mathrm{M}]_{\mathrm{o}} /[\mathrm{l}]_{\mathrm{o}}} & \text { conv }^{a}(\%) & M_{\mathrm{n}}{ }^{b} & M_{\mathrm{w}} / M_{\mathrm{n}}{ }^{b} \\ 1 & 50 & 91 & 8800 & 1.07 \\ 2 & 100 & 90 & 15500 & 1.04 \\ 3 & 200 & 90 & 26000 & 1.03\end{array}$

Table S2. $[\mathrm{M}]_{0} /[\mathrm{I}]$ o screen for the DBU/5CTU catalyzed ROP of VL. Reaction conditions: VL (2 M), benzyl alcohol (2.0 mol \%) in benzene- $d_{6}$ catalyzed by DBU/5CTU (2.5 mol \% each). ${ }^{a}$ Conversion tracked by ${ }^{1} \mathrm{HNMR}$. ${ }^{b}$ Calculated by GPC on the basis of polystyrene standards eluted by $\mathrm{CH}_{2} \mathrm{Cl}_{2}$.

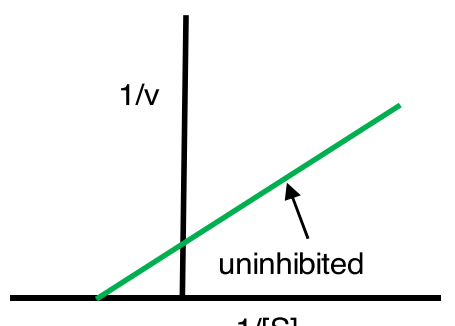

1/[S]
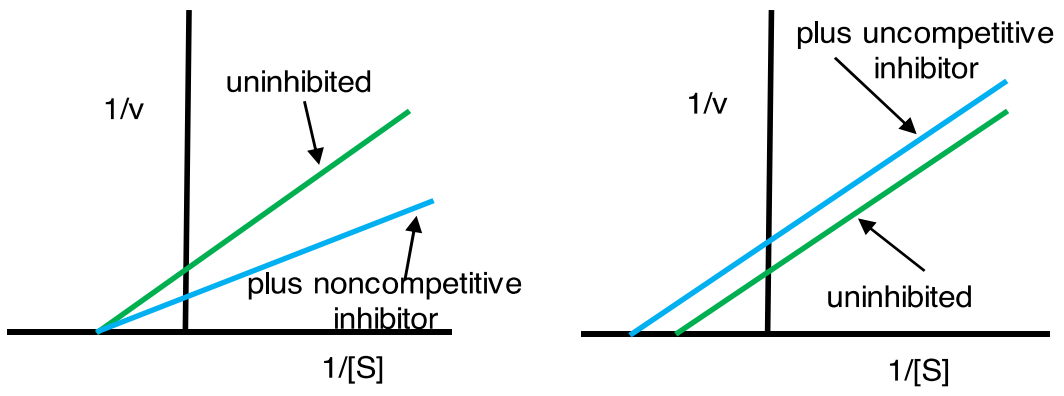

Figure S3. Types of Michaelis-Menten enzyme kinetics inhibition displayed as LinweaverBurke plots. 


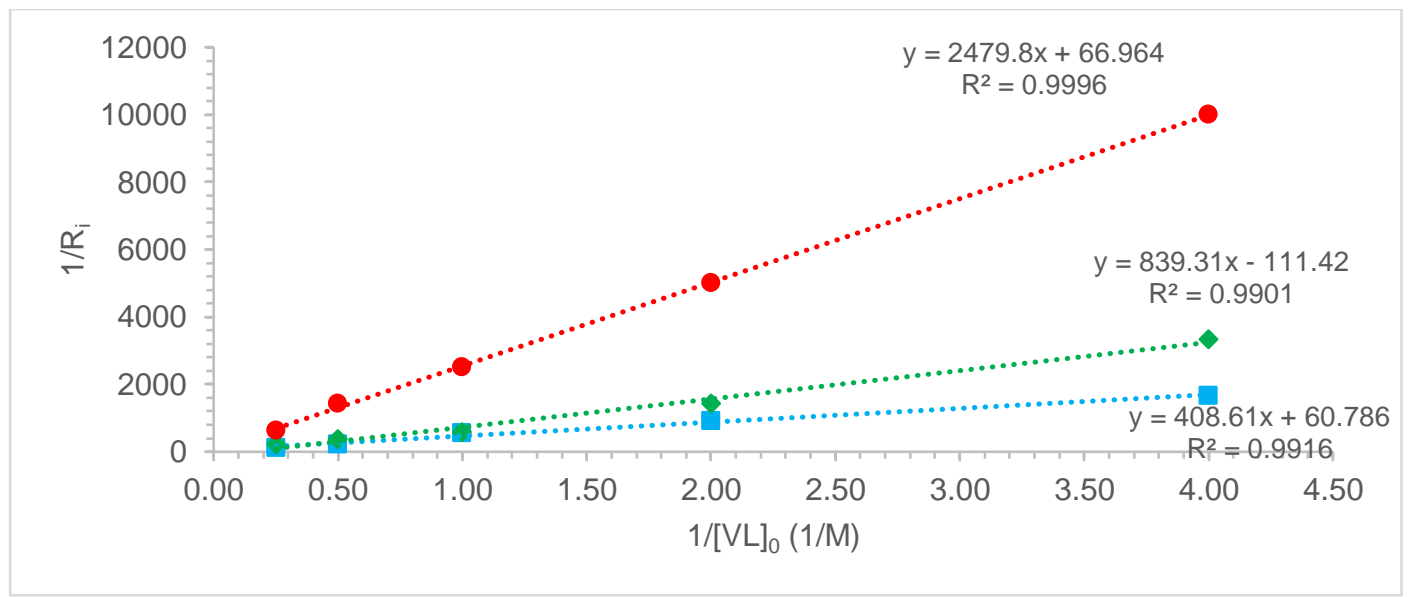

Figure S4. Linweaver-Burke plot of the MTBD (10 mM, squares; $100 \mathrm{mM}$, diamonds; $500 \mathrm{mM}$, circles) and 5CTU catalyzed polymerization of VL. Reaction conditions: VL $(0.25,0.5,1.0,2.0$, $4.0 \mathrm{M})$, benzyl alcohol $(5 \mathrm{mM})$ in benzene- $d_{6}$ catalyzed by MTBD $(10,100,500 \mathrm{mM}) 5 \mathrm{CTU}(5$ $\mathrm{mM})$. Conversion tracked by NMR.
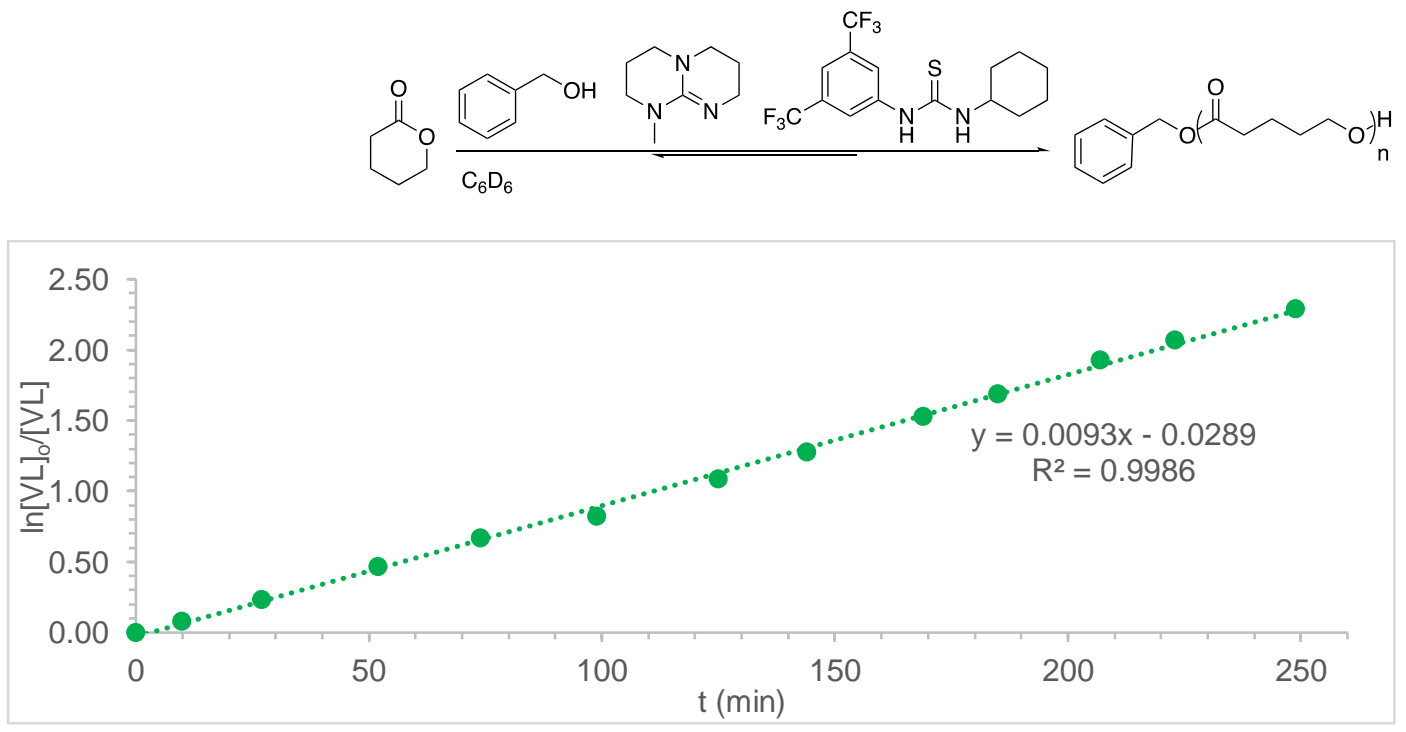

Figure S5. First-order evolution of [VL] versus time for the MTBD/CyTU catalyzed ROP of VL. Reaction conditions: VL ( $2 \mathrm{M})$, initiated by benzyl alcohol $(2.0 \mathrm{~mol} \%)$ in benzene- $d_{6}$ catalyzed by MTBD/CyTU (2.5 mol \% each). Conversion tracked by ${ }^{1} \mathrm{HNMR}$. 

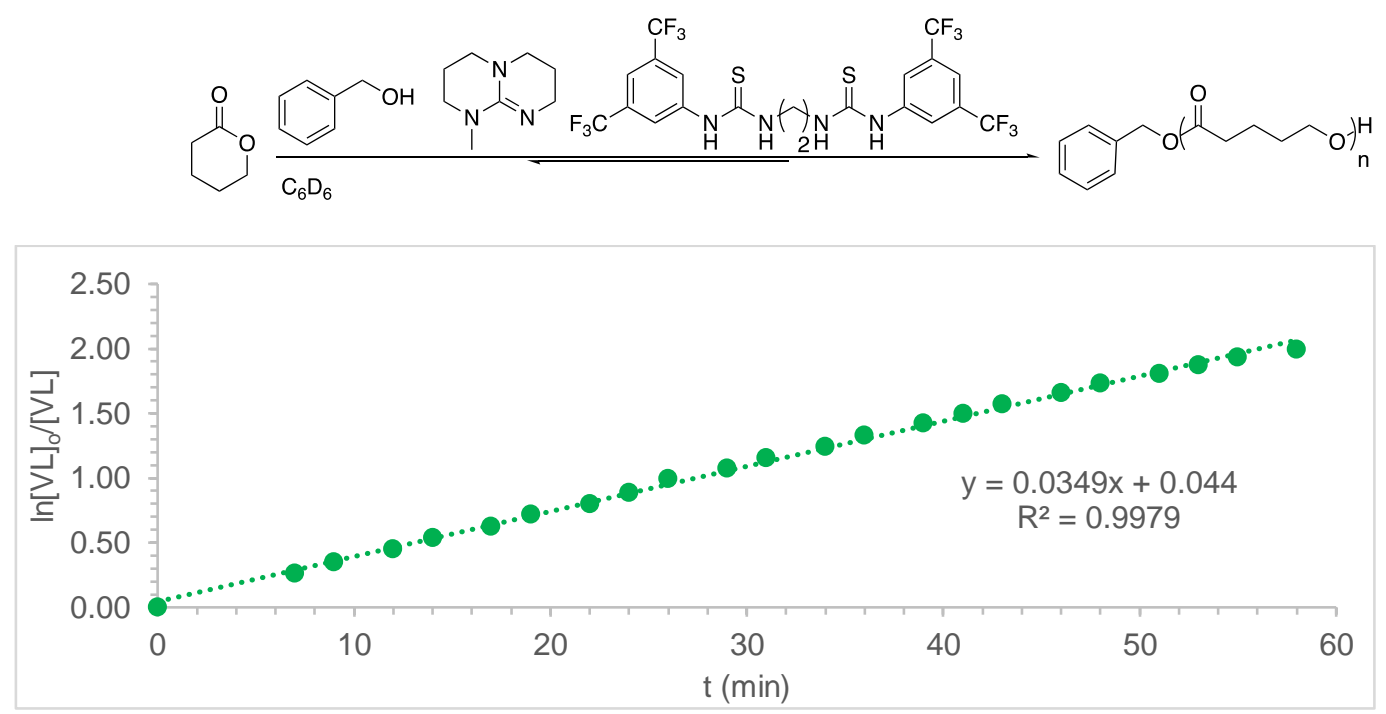

Figure S6. First-order evolution of [VL] versus time for the MTBD/2CTU catalyzed ROP of VL. Reaction conditions: VL (2 M), initiated by benzyl alcohol $(2.0 \mathrm{~mol} \%)$ in benzene- $d_{6}$ catalyzed by MTBD/2CTU (2.5 mol \% each). Conversion tracked by ${ }^{1} \mathrm{HNMR}$.
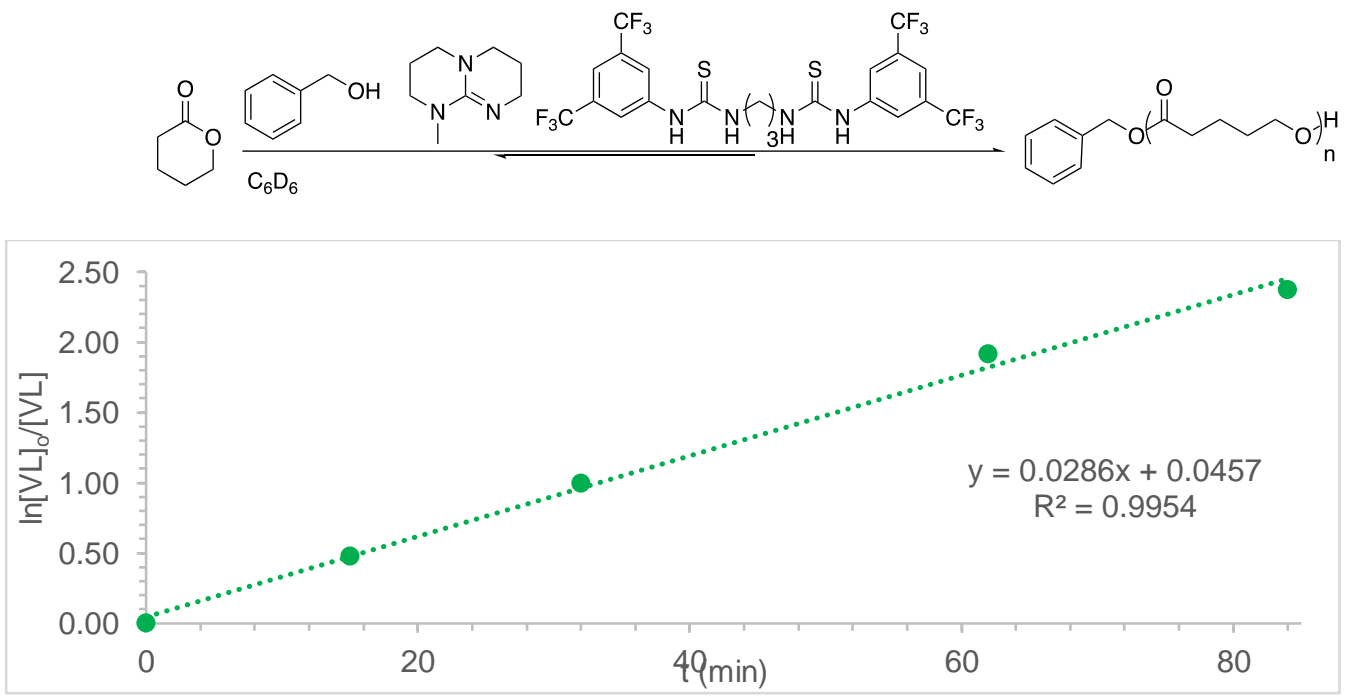

Figure S7. First-order evolution of [VL] versus time for the MTBD/3CTU catalyzed ROP of VL. Reaction conditions: VL (2 M), initiated by benzyl alcohol $(2.0 \mathrm{~mol} \%)$ in benzene- $d_{6}$ catalyzed by MTBD/3CTU (2.5 mol \% each). Conversion tracked by ${ }^{1} \mathrm{HNMR}$. 

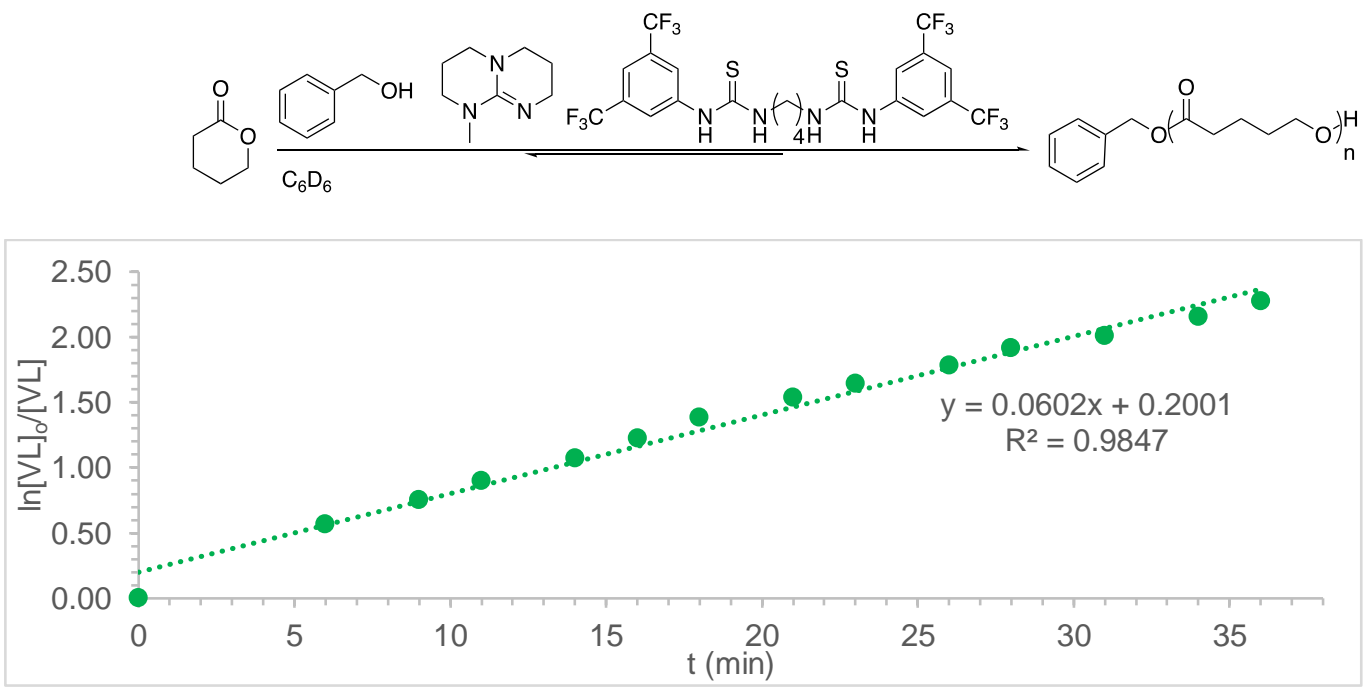

Figure S8. First-order evolution of [VL] versus time for the MTBD/4CTU catalyzed ROP of VL. Reaction conditions: VL $(2 \mathrm{M})$, initiated by benzyl alcohol $(2.0 \mathrm{~mol} \%)$ in benzene- $d_{6}$ catalyzed by MTBD/4CTU (2.5 mol \% each). Conversion tracked by ${ }^{1} \mathrm{HNMR}$.
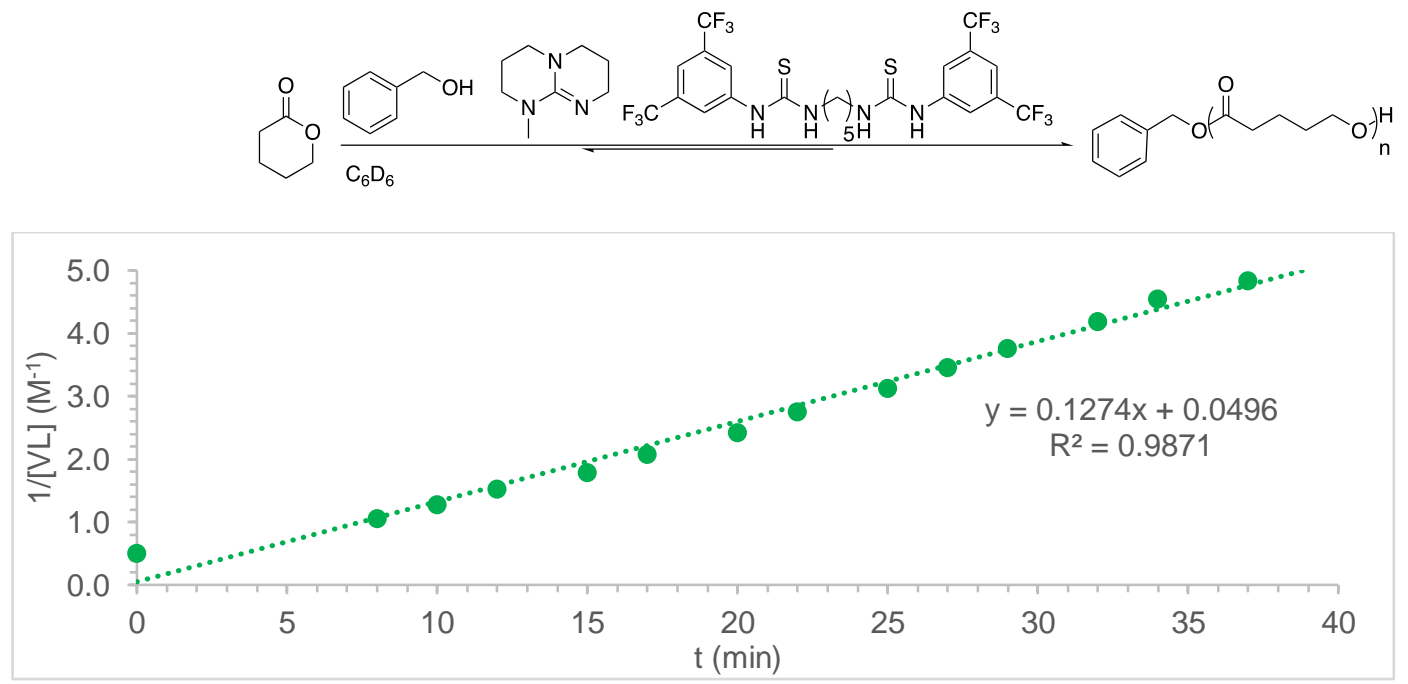

Figure S9. Second-order evolution of [VL] versus time for the MTBD/5CTU catalyzed ROP of VL. Reaction conditions: VL (2 M), initiated by benzyl alcohol $(2.0 \mathrm{~mol} \%)$ in benzene- $d_{6}$ catalyzed by MTBD/5CTU ( $2.5 \mathrm{~mol} \%$ each). Conversion tracked by ${ }^{1} \mathrm{HNMR}$. 

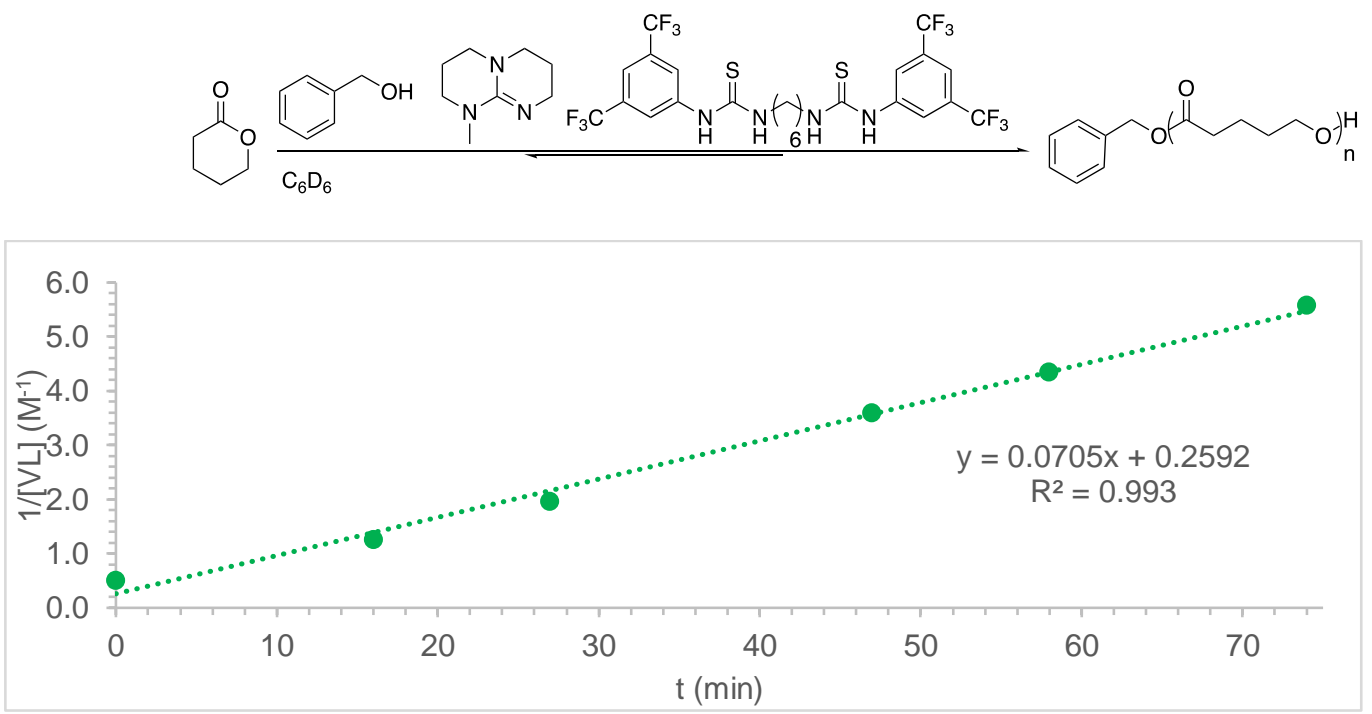

Figure S10. Second-order evolution of [VL] versus time for the MTBD/6CTU catalyzed ROP of VL. Reaction conditions: VL (2 M), initiated by benzyl alcohol $(2.0 \mathrm{~mol} \%)$ in benzene- $d_{6}$ catalyzed by MTBD/6CTU ( $2.5 \mathrm{~mol} \%$ each). Conversion tracked by ${ }^{1} \mathrm{HNMR}$.
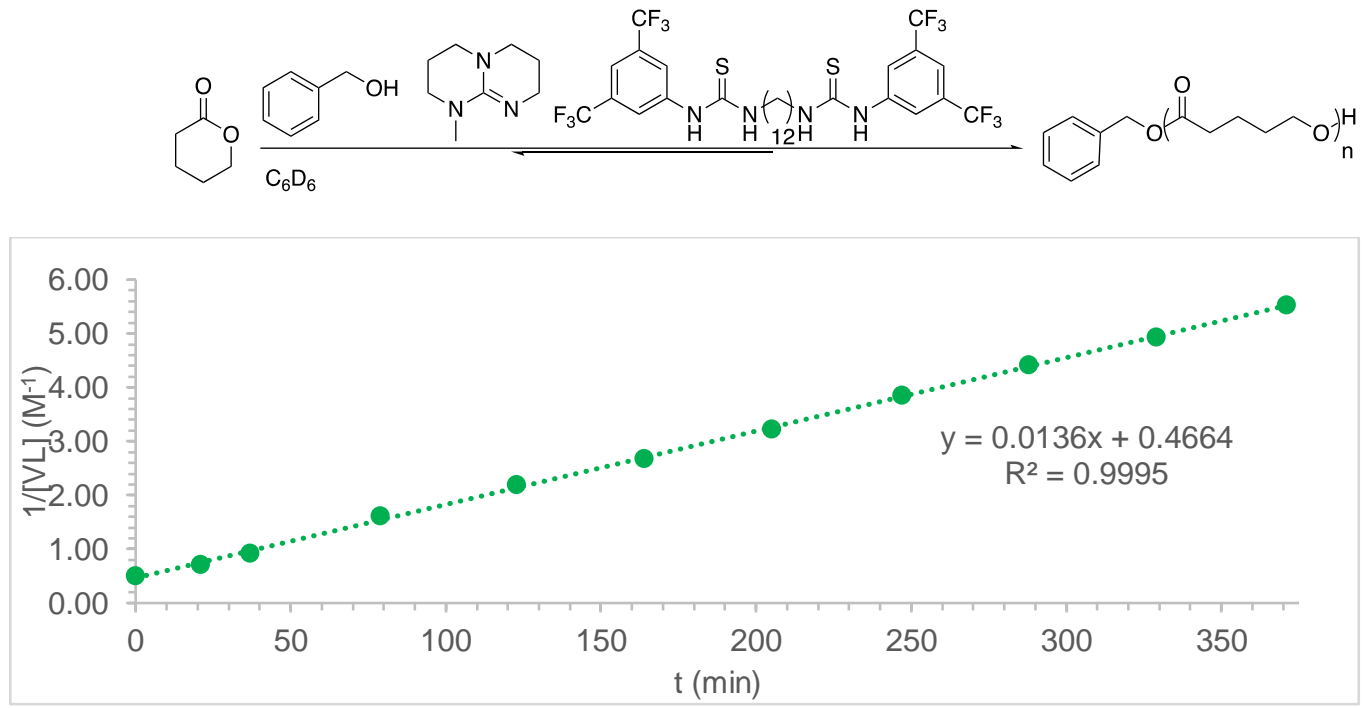

Figure S11. Second-order evolution of [VL] versus time for the MTBD/12CTU catalyzed ROP of VL. Reaction conditions: VL (2 M), initiated by benzyl alcohol $(2.0 \mathrm{~mol} \%)$ in benzene-d6 catalyzed by MTBD/12CTU (2.5 mol \% each). Conversion tracked by ${ }^{1} \mathrm{HNMR}$. 

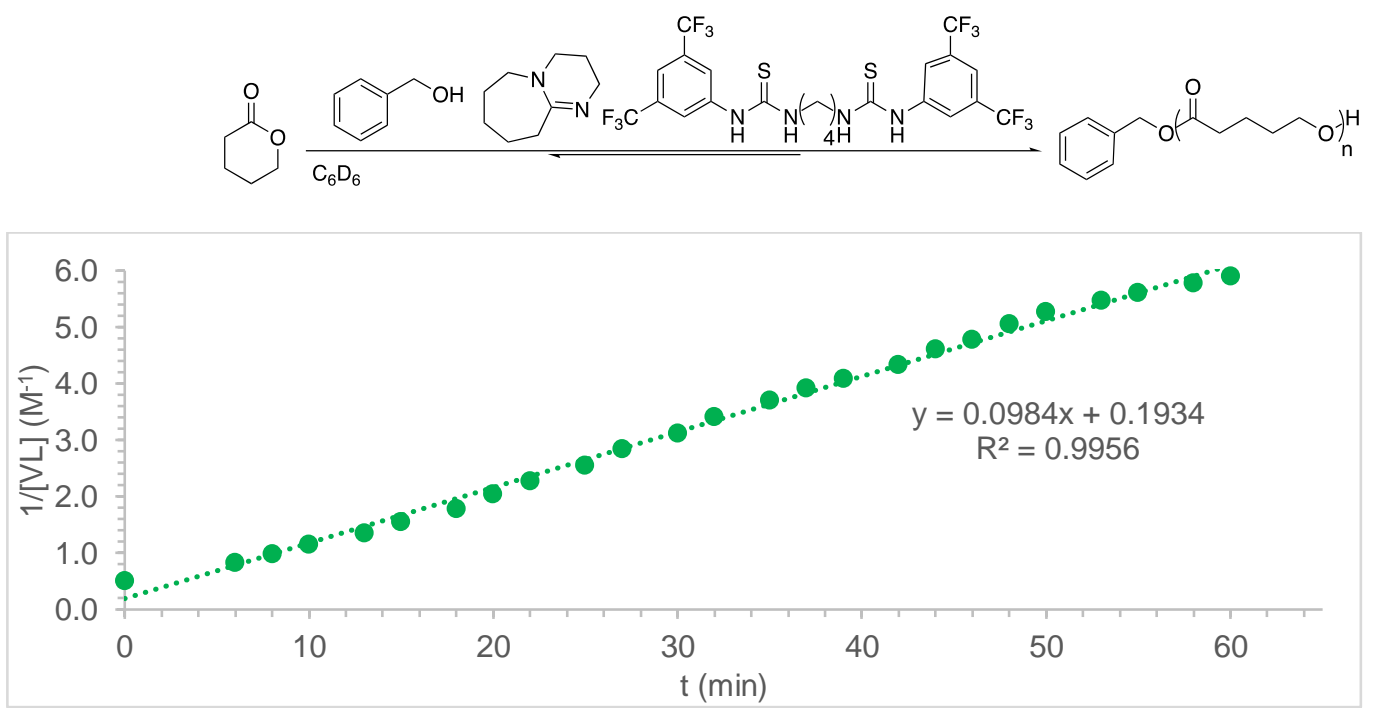

Figure S12. Second-order evolution of [VL] versus time for the DBU/4CTU catalyzed ROP of VL. Reaction conditions: VL (2 M), initiated by benzyl alcohol $(2.0 \mathrm{~mol} \%)$ in benzene- $d_{6}$ catalyzed by DBU/4CTU (2.5 mol \% each). Conversion tracked by ${ }^{1} \mathrm{HNMR}$.
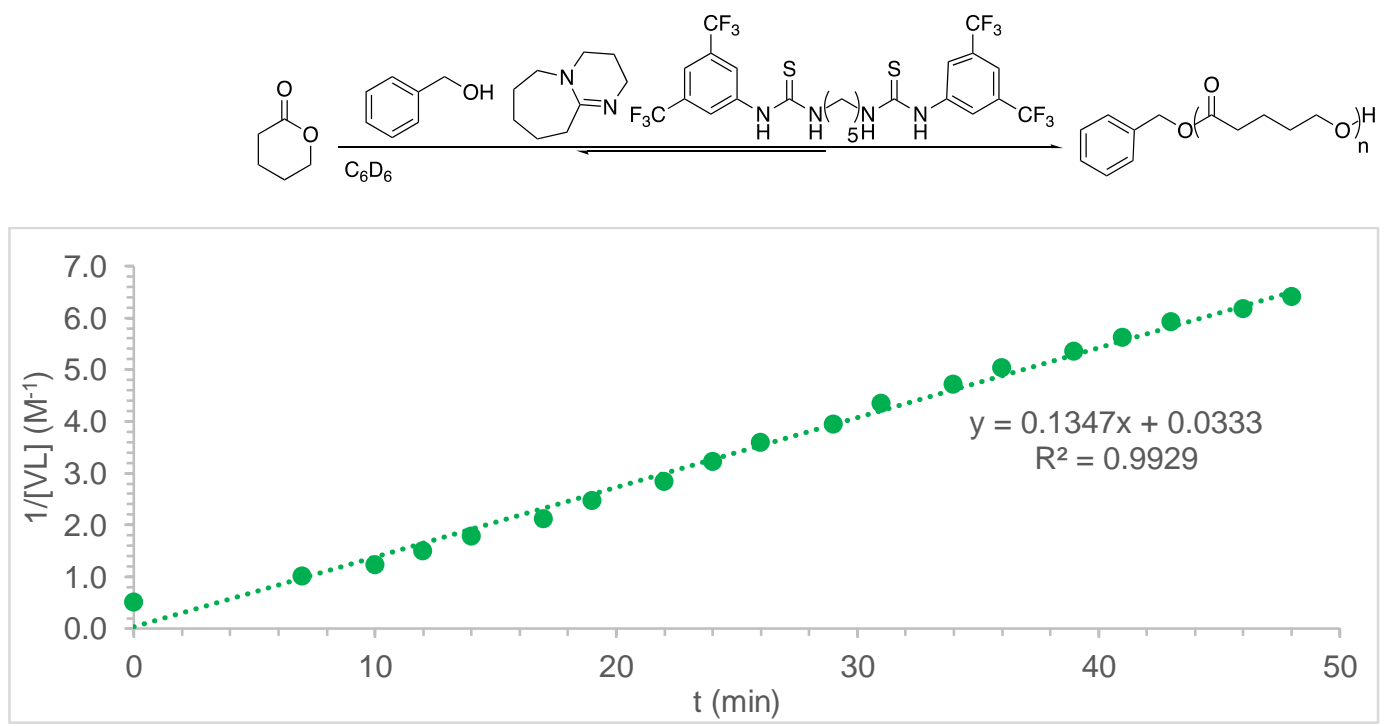

Figure S13. Second-order evolution of [VL] versus time for the DBU/5CTU catalyzed ROP of VL. Reaction conditions: VL (2 M), initiated by benzyl alcohol $(2.0 \mathrm{~mol} \%)$ in benzene- $d_{6}$ catalyzed by DBU/5CTU (2.5 mol \% each). Conversion tracked by ${ }^{1} \mathrm{HNMR}$. 

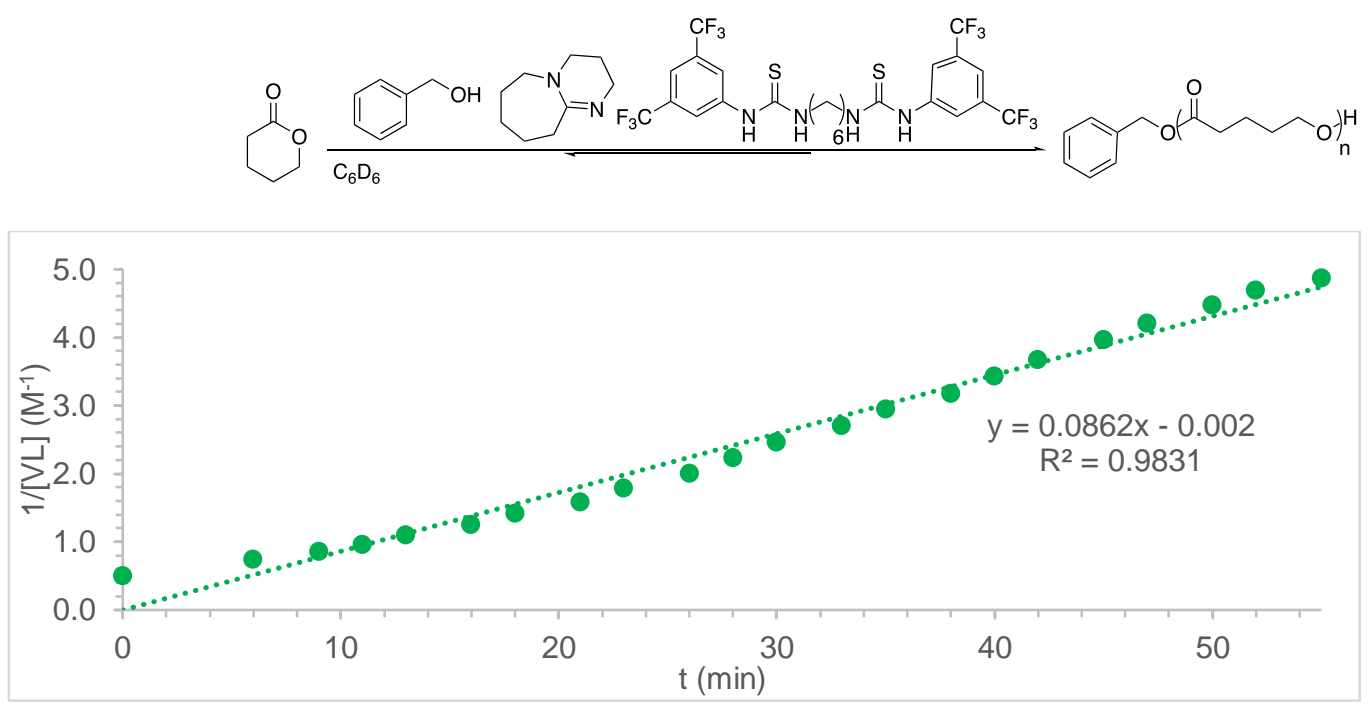

Figure S14. Second-order evolution of [VL] versus time for the DBU/6CTU catalyzed ROP of VL. Reaction conditions: VL (2 M), initiated by benzyl alcohol $(2.0 \mathrm{~mol} \%)$ in benzene- $d_{6}$ catalyzed by DBU/6CTU (2.5 mol \% each). Conversion tracked by ${ }^{1} \mathrm{HNMR}$.
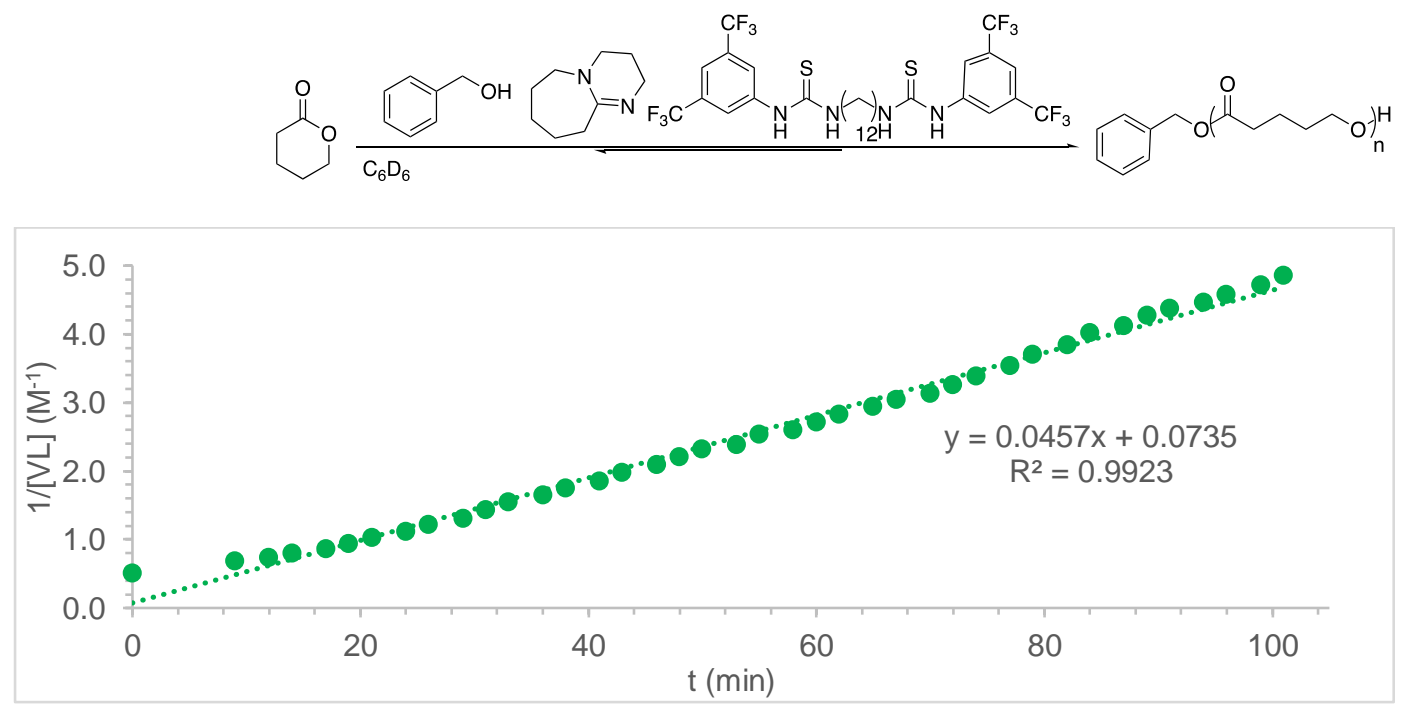

Figure S15. Second-order evolution of [VL] versus time for the DBU/12CTU catalyzed ROP of VL. Reaction conditions: VL (2 M), initiated by benzyl alcohol $(2.0 \mathrm{~mol} \%)$ in benzene- $d_{6}$ catalyzed by DBU/12CTU ( $2.5 \mathrm{~mol} \%$ each). Conversion tracked by ${ }^{1} \mathrm{HNMR}$. 

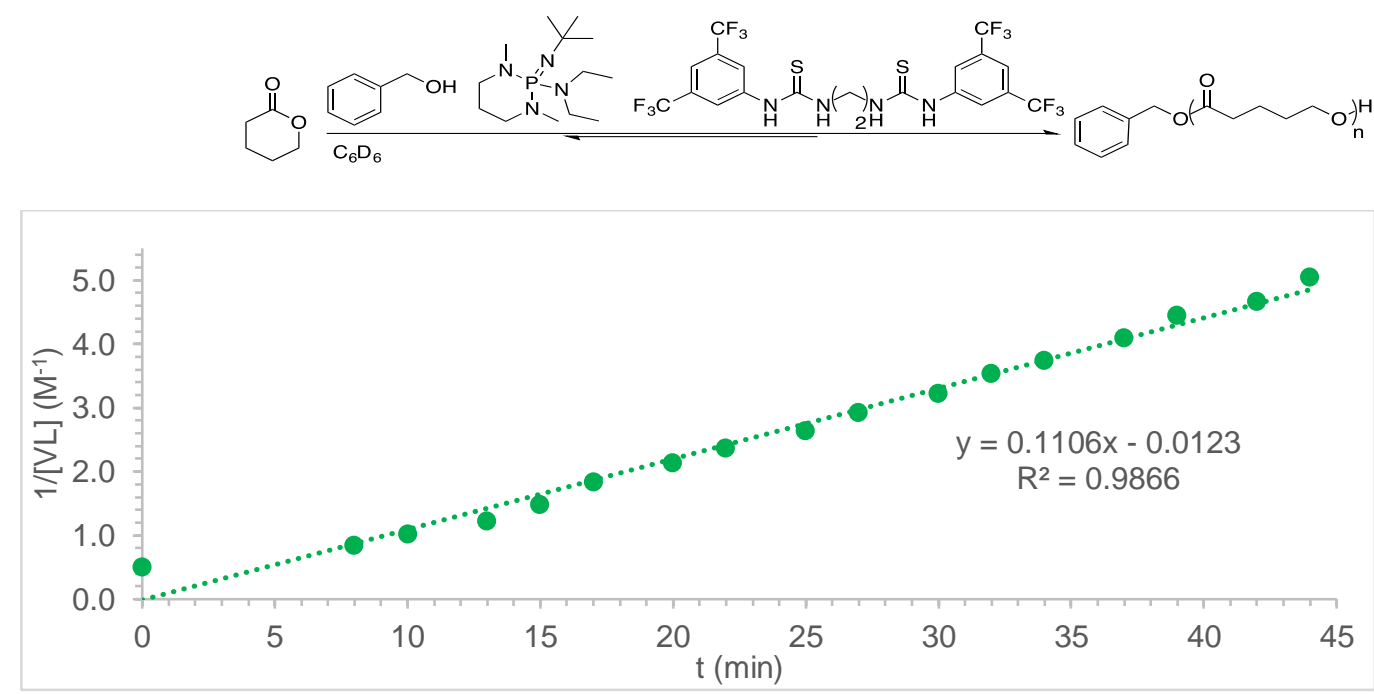

Figure S16. Second-order evolution of [VL] versus time for the BEMP/2CTU catalyzed ROP of VL. Reaction conditions: VL (2 M), initiated by benzyl alcohol $(2.0 \mathrm{~mol} \%)$ in benzene- $d_{6}$ catalyzed by BEMP/2CTU ( $2.5 \mathrm{~mol} \%$ each). Conversion tracked by ${ }^{1} \mathrm{HNMR}$.
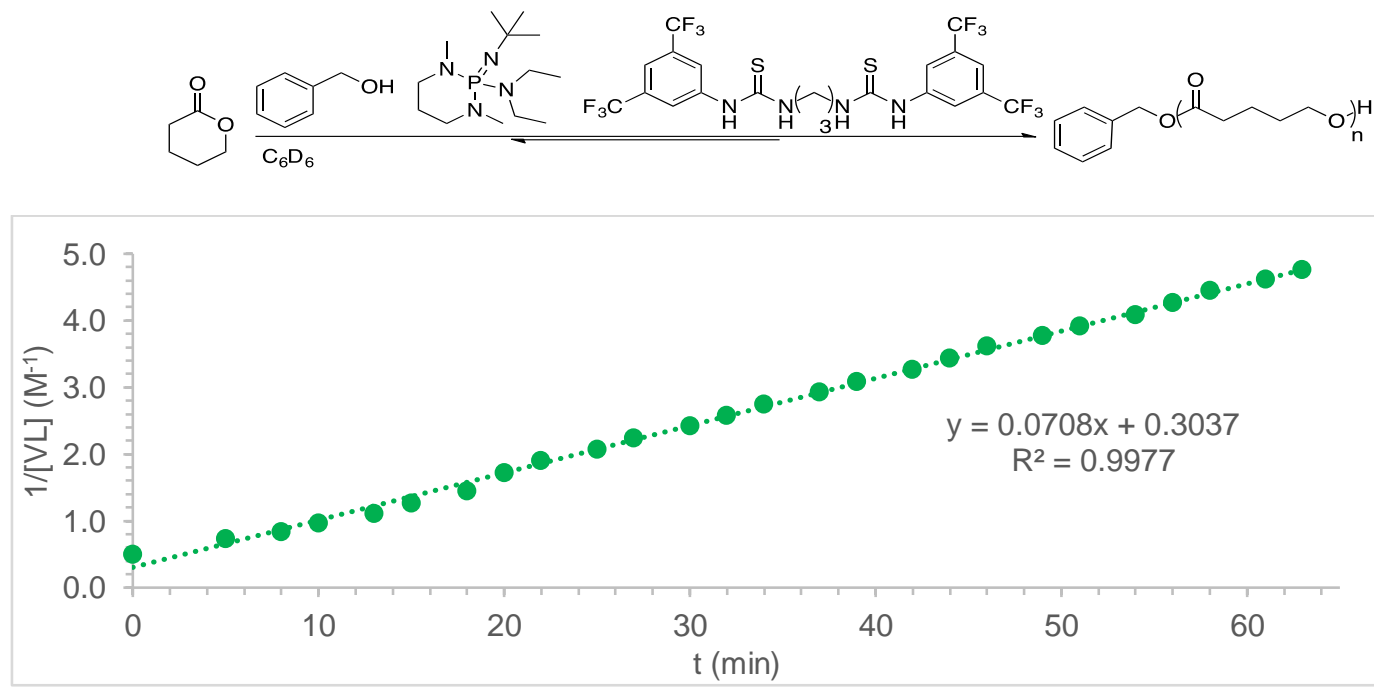

Figure S17. Second-order evolution of [VL] versus time for the BEMP/3CTU catalyzed ROP of VL. Reaction conditions: VL (2 M), initiated by benzyl alcohol $(2.0 \mathrm{~mol} \%)$ in benzene- $d_{6}$ catalyzed by BEMP/3CTU ( $2.5 \mathrm{~mol} \%$ each). Conversion tracked by ${ }^{1} \mathrm{HNMR}$. 

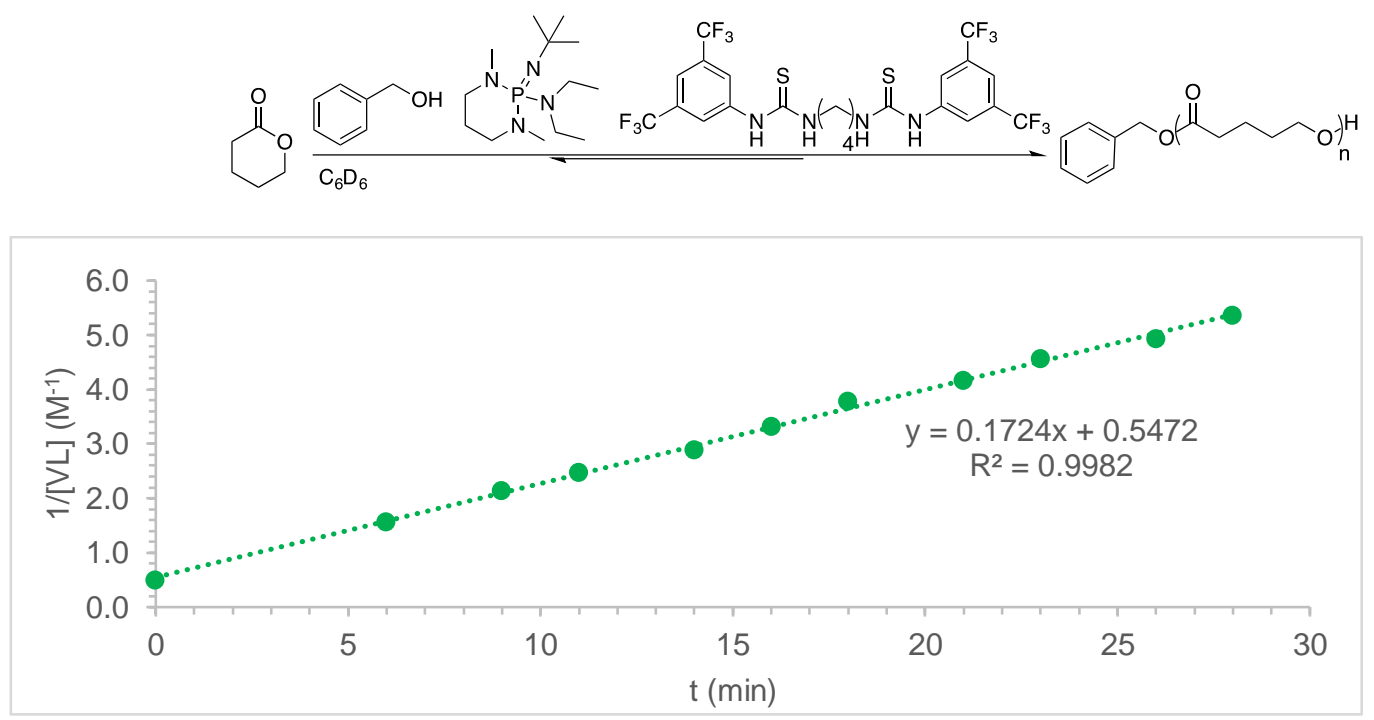

Figure S18. Second-order evolution of [VL] versus time for the BEMP/4CTU catalyzed ROP of VL. Reaction conditions: VL (2 M), initiated by benzyl alcohol $(2.0 \mathrm{~mol} \%)$ in benzene- $d_{6}$ catalyzed by BEMP/4CTU (2.5 mol \% each). Conversion tracked by ${ }^{1} \mathrm{HNMR}$.
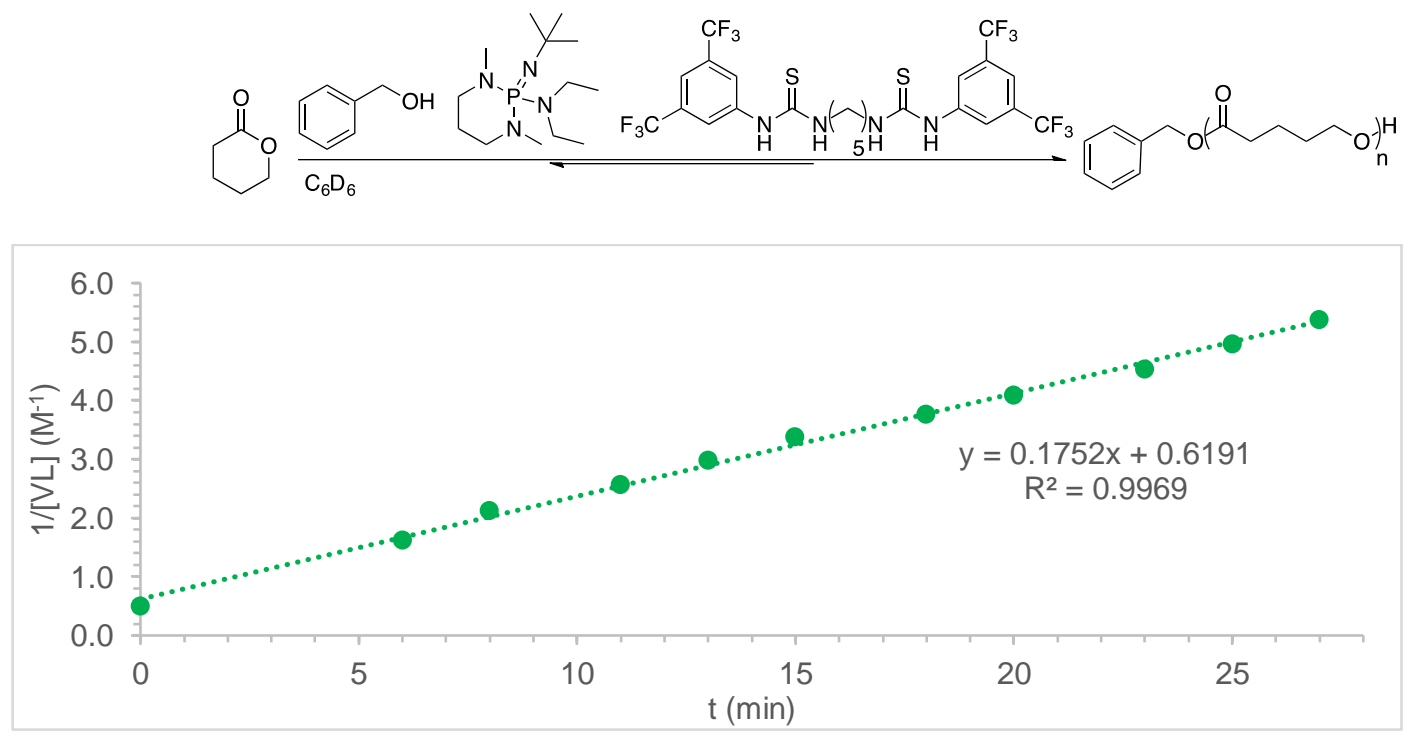

Figure S19. Second-order evolution of [VL] versus time for the BEMP/5CTU catalyzed ROP of VL. Reaction conditions: VL (2 M), initiated by benzyl alcohol $(2.0 \mathrm{~mol} \%)$ in benzene- $d_{6}$ catalyzed by BEMP/5CTU (2.5 mol \% each). Conversion tracked by ${ }^{1} \mathrm{HNMR}$. 

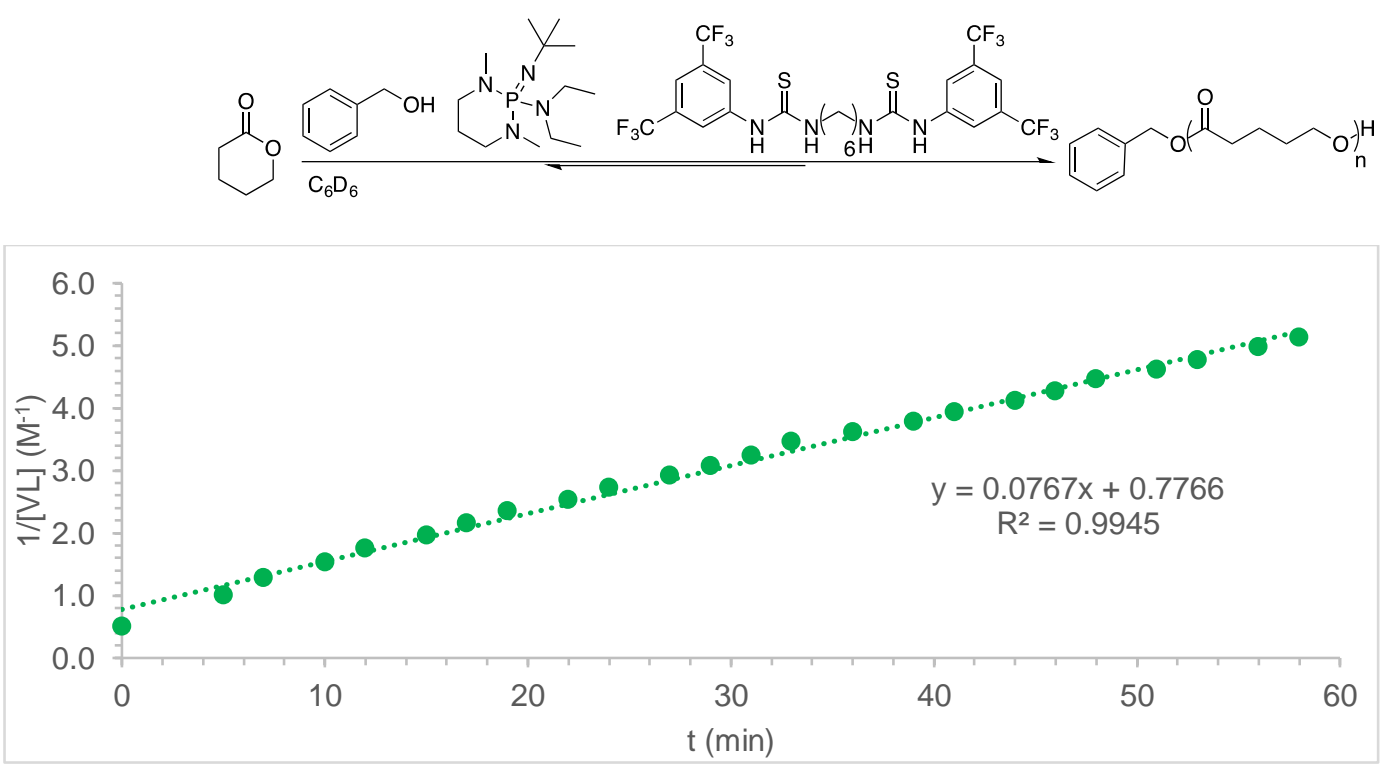

Figure S20. Second-order evolution of [VL] versus time for the BEMP/6CTU catalyzed ROP of VL. Reaction conditions: VL (2 M), initiated by benzyl alcohol $(2.0 \mathrm{~mol} \%)$ in benzene- $d_{6}$ catalyzed by BEMP/6CTU (2.5 mol \% each). Conversion tracked by ${ }^{1} \mathrm{HNMR}$.
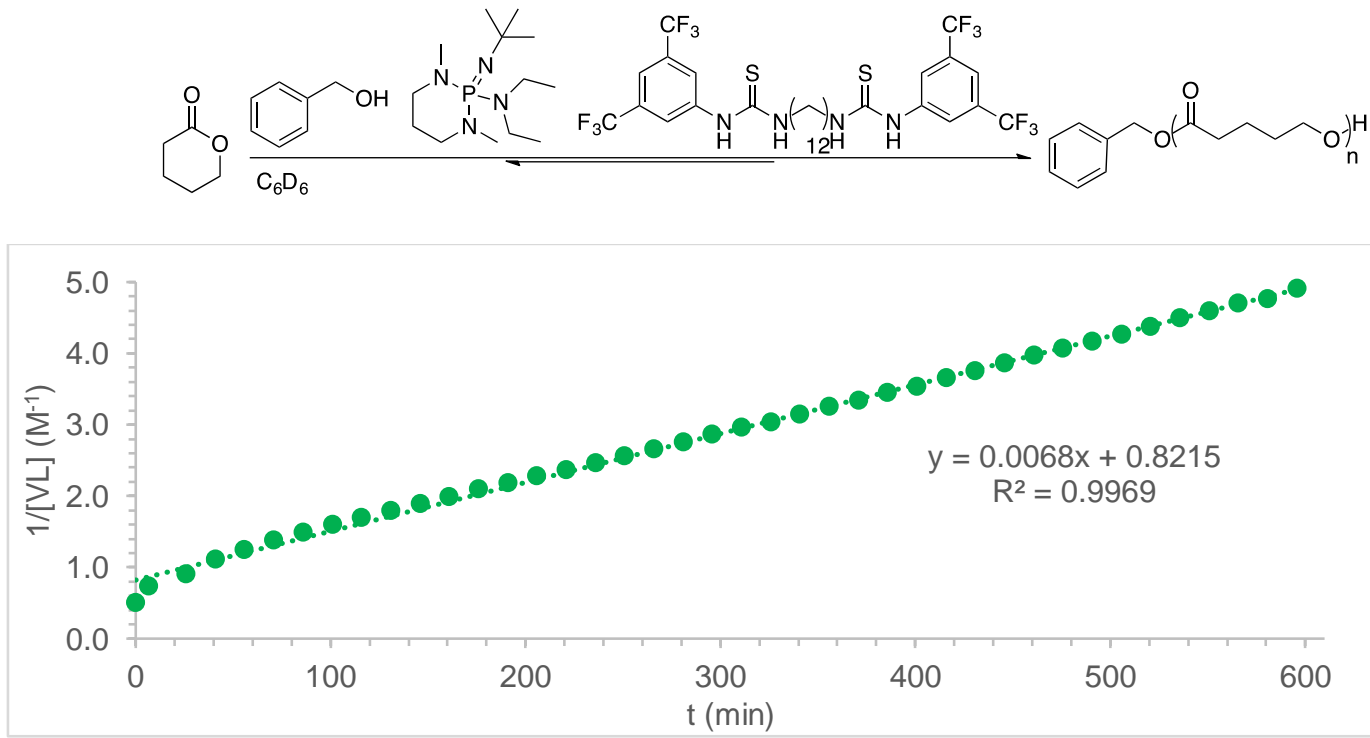

Figure S21. Second-order evolution of [VL] versus time for the BEMP/12CTU catalyzed ROP of VL. Reaction conditions: VL (2 M), initiated by benzyl alcohol $(2.0 \mathrm{~mol} \%)$ in benzene-d6 catalyzed by BEMP/12CTU (2.5 mol \% each). Conversion tracked by ${ }^{1} \mathrm{HNMR}$. 


$\begin{array}{llcc}\text { entry } & \text { nCTU } & \text { initial rate } & \text { relative initial rate } \\ 1 & \text { CyTU } & 1.55 \mathrm{E}-02 & 1.00 \\ 2 & 2 \mathrm{CTU} & 9.35 \mathrm{E}-02 & 6.03 \\ 3 & 3 \mathrm{CTU} & 9.53 \mathrm{E}-02 & 6.15 \\ 4 & 4 \mathrm{CTU} & 1.78 \mathrm{E}-01 & 11.50 \\ 5 & 5 \mathrm{CTU} & 2.00 \mathrm{E}-01 & 12.92 \\ 6 & 6 \mathrm{CTU} & 1.79 \mathrm{E}-01 & 11.52 \\ 7 & 12 \mathrm{CTU} & 2.98 \mathrm{E}-02 & 1.92\end{array}$

Table S3. Initial rate and relative initial rate of the BEMP/nCTU catalyzed ROP of VL. Reaction conditions: VL $(2 \mathrm{M})$, initiated by benzyl alcohol $(2.0 \mathrm{~mol} \%)$ in benzene- $d_{6}$ catalyzed by $\mathrm{BEMP} / \mathrm{nCTU}(2.5 \mathrm{~mol} \%$ each).

$\begin{array}{llcc}\text { entry } & \text { TU } & \text { initial rate }(\mathrm{M} / \mathrm{min}) & \text { relative initial rate } \\ 1 & \text { CyTU } & 1.33 \mathrm{E}-02 & 1.00 \\ 2 & \text { 2CTU } & 5.54 \mathrm{E}-02 & 4.17 \\ 3 & 3 \mathrm{CTU} & 3.92 \mathrm{E}-02 & 2.95 \\ 4 & \text { 4CTU } & 1.22 \mathrm{E}-01 & 9.16 \\ 5 & 5 \mathrm{CTU} & 1.24 \mathrm{E}-01 & 9.32 \\ 6 & \text { 6CTU } & 5.65 \mathrm{E}-02 & 4.25 \\ 7 & 12 \mathrm{CTU} & 2.50 \mathrm{E}-02 & 1.88\end{array}$

Table S4. Initial rate and relative initial rate of the MTBD/nCTU catalyzed ROP of VL. Reaction conditions: VL $(2 \mathrm{M})$, initiated by benzyl alcohol $(2.0 \mathrm{~mol} \%)$ in benzene- $d_{6}$ catalyzed by MTBD $/$ nCTU (2.5 mol \% each).

$\begin{array}{lccc}\text { entry } & \text { TU } & \text { initial rate }(\mathrm{M} / \mathrm{min}) & \text { relative initial rate } \\ 1 & \text { CyTU } & 1.47 \mathrm{E}-02 & 1.00 \\ 2 & \text { 2CTU } & 4.37 \mathrm{E}-02 & 2.97 \\ 3 & 3 \mathrm{CTU} & 6.24 \mathrm{E}-02 & 4.24 \\ 4 & 4 \mathrm{CTU} & 1.66 \mathrm{E}-01 & 11.29 \\ 5 & 5 \mathrm{CTU} & 1.23 \mathrm{E}-01 & 8.38 \\ 6 & 6 \mathrm{CTU} & 8.77 \mathrm{E}-02 & 5.97 \\ 7 & \text { 12CTU } & 4.90 \mathrm{E}-02 & 3.33\end{array}$

Table S5. Initial rate and relative initial rate of the DBU/nCTU catalyzed ROP of VL. Reaction conditions: VL $(2 \mathrm{M})$, initiated by benzyl alcohol $(2.0 \mathrm{~mol} \%)$ in benzene- $d_{6}$ catalyzed by $\mathrm{DBU} / \mathrm{nCTU}(2.5 \mathrm{~mol} \%$ each). 


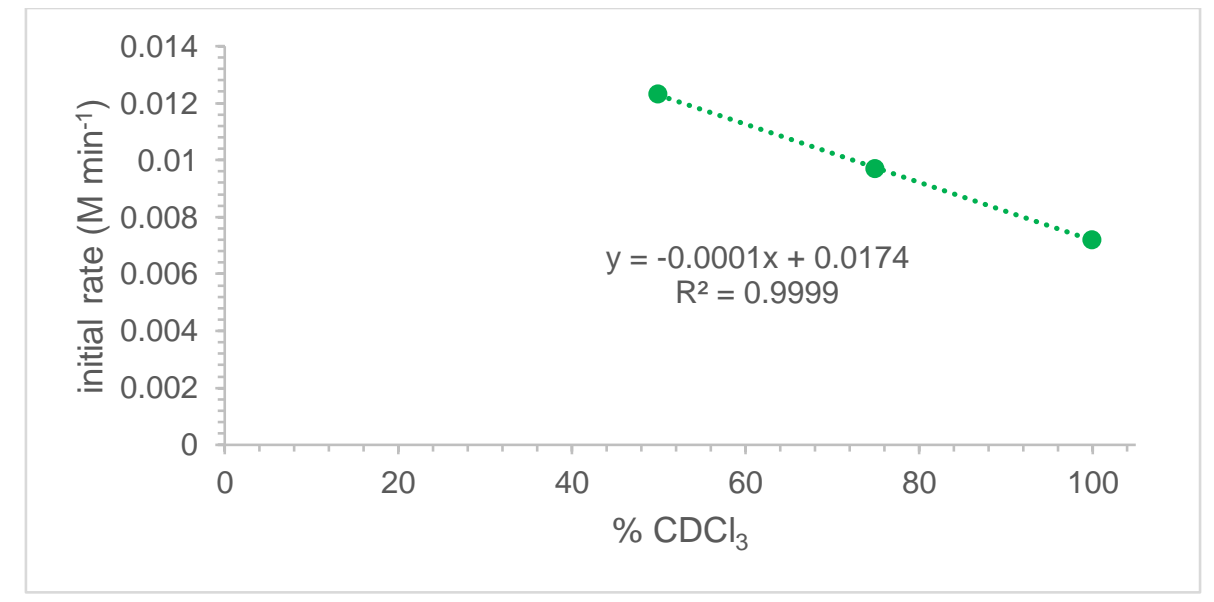

Figure S22. $k_{i e}$ of the DBU/5CTU catalyzed ROP of VL. Reaction conditions: VL (2 M), initiated by benzyl alcohol $(2.0 \mathrm{~mol} \%)$ in chloroform and chloroform- $d$ by varying ratio of solvent $\left(100 \% \mathrm{CDCl}_{3}, 75 \% / 25 \% \mathrm{CDCl}_{3} / \mathrm{CHCl}_{3}, 50 \% / 50 \% \mathrm{CDCl}_{3} / \mathrm{CHCl}_{3}\right)$ catalyzed by $\mathrm{DBU} / 5 \mathrm{CTU}\left(2.5 \mathrm{~mol} \%\right.$ each). Conversion tracked by ${ }^{1} \mathrm{HNMR}$. 

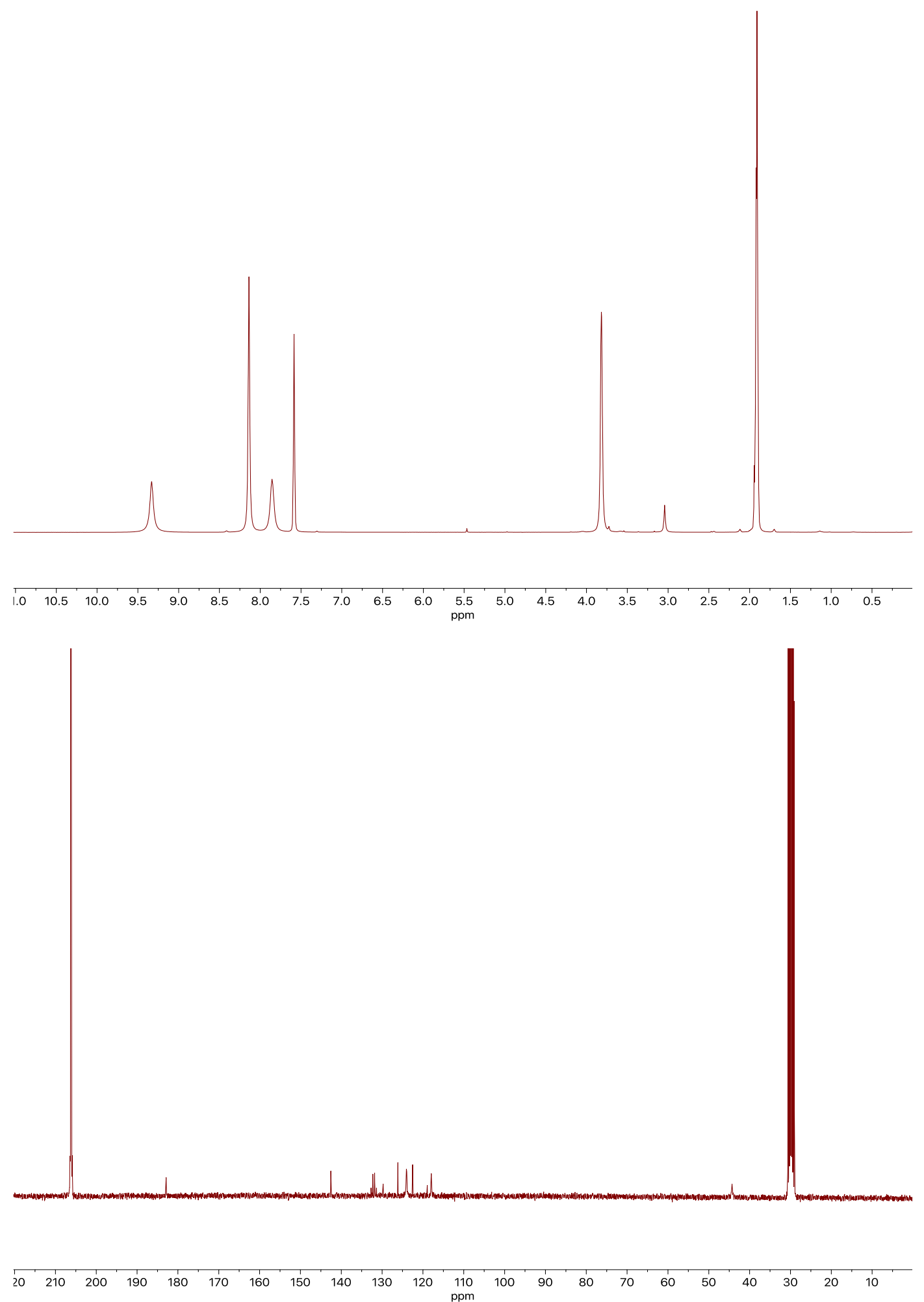

Figure S23. Top) ${ }^{1} \mathrm{H}$ NMR of $2 \mathrm{CTU}$ in acetone- $d_{6}$. Bottom) ${ }^{13} \mathrm{C}$ NMR of $2 \mathrm{CTU}$ in acetone- $d_{6}$. 


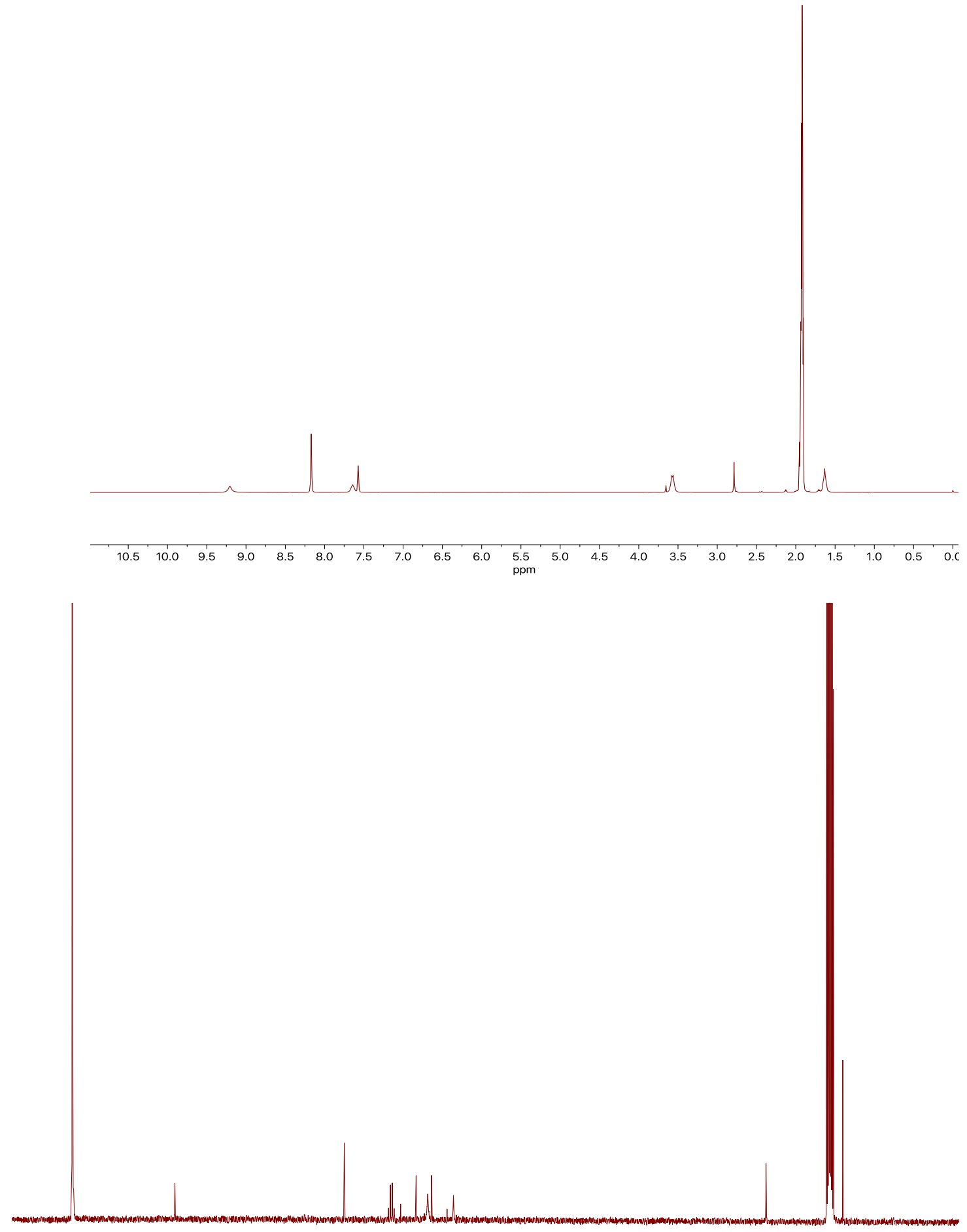

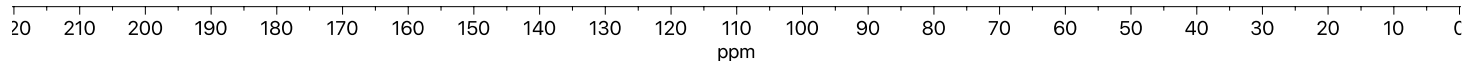

Figure S24. Top) ${ }^{1} \mathrm{H}$ NMR of $4 \mathrm{CTU}$ in acetone- $d_{6}$. Bottom) ${ }^{13} \mathrm{C}$ NMR of $4 \mathrm{CTU}$ in acetone- $d_{6}$. 

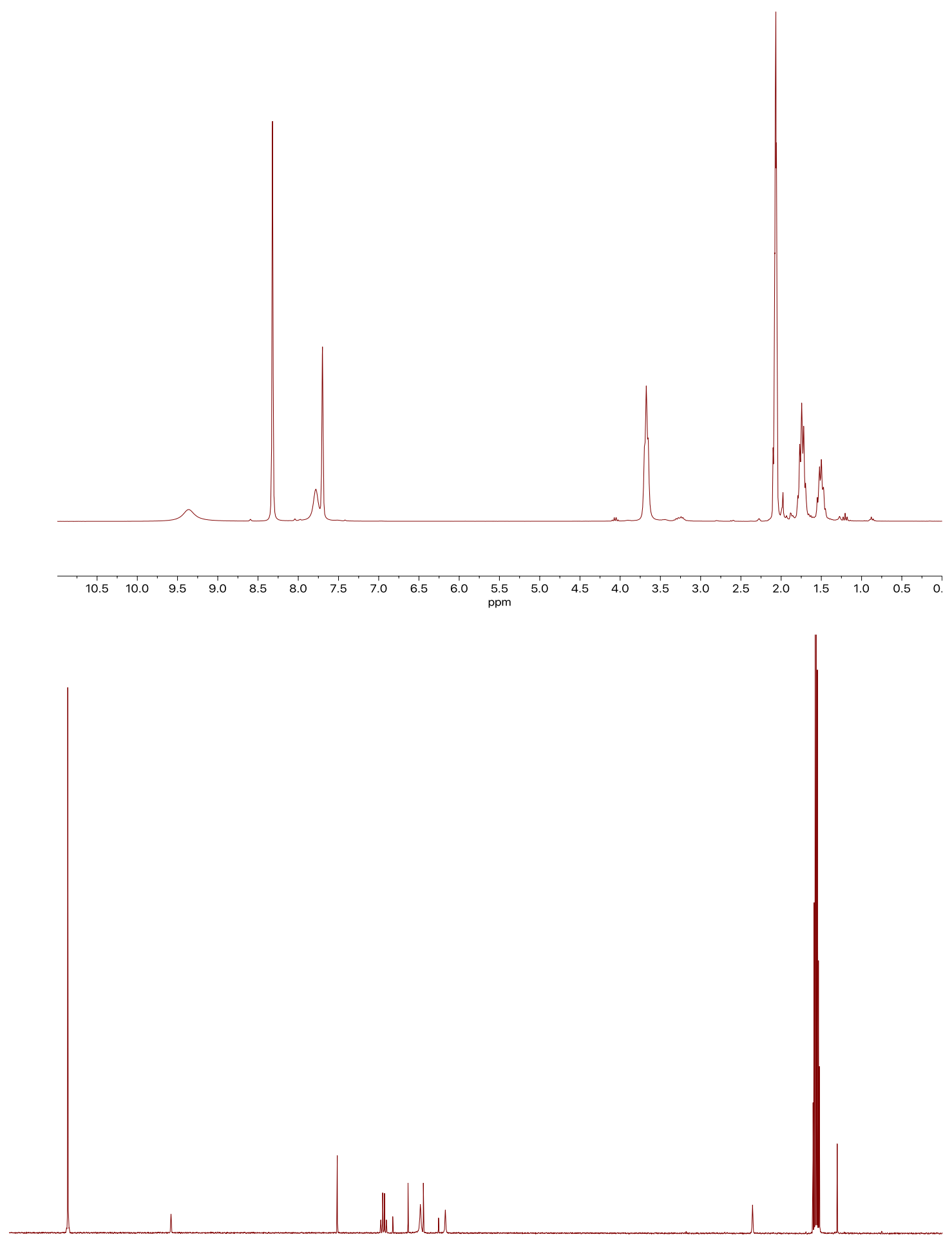

$\begin{array}{llllllllllllllllllllllll}20 & 210 & 200 & 190 & 180 & 170 & 160 & 150 & 140 & 130 & 120 & 110 & 100 & 90 & 80 & 70 & 60 & 50 & 40 & 30 & 20 & 10 & 10\end{array}$

Figure S25. Top) ${ }^{1} \mathrm{H}$ NMR of $5 \mathrm{CTU}$ in acetone- $d_{6}$. Bottom) ${ }^{13} \mathrm{C}$ NMR of $5 \mathrm{CTU}$ in acetone- $d_{6}$. 


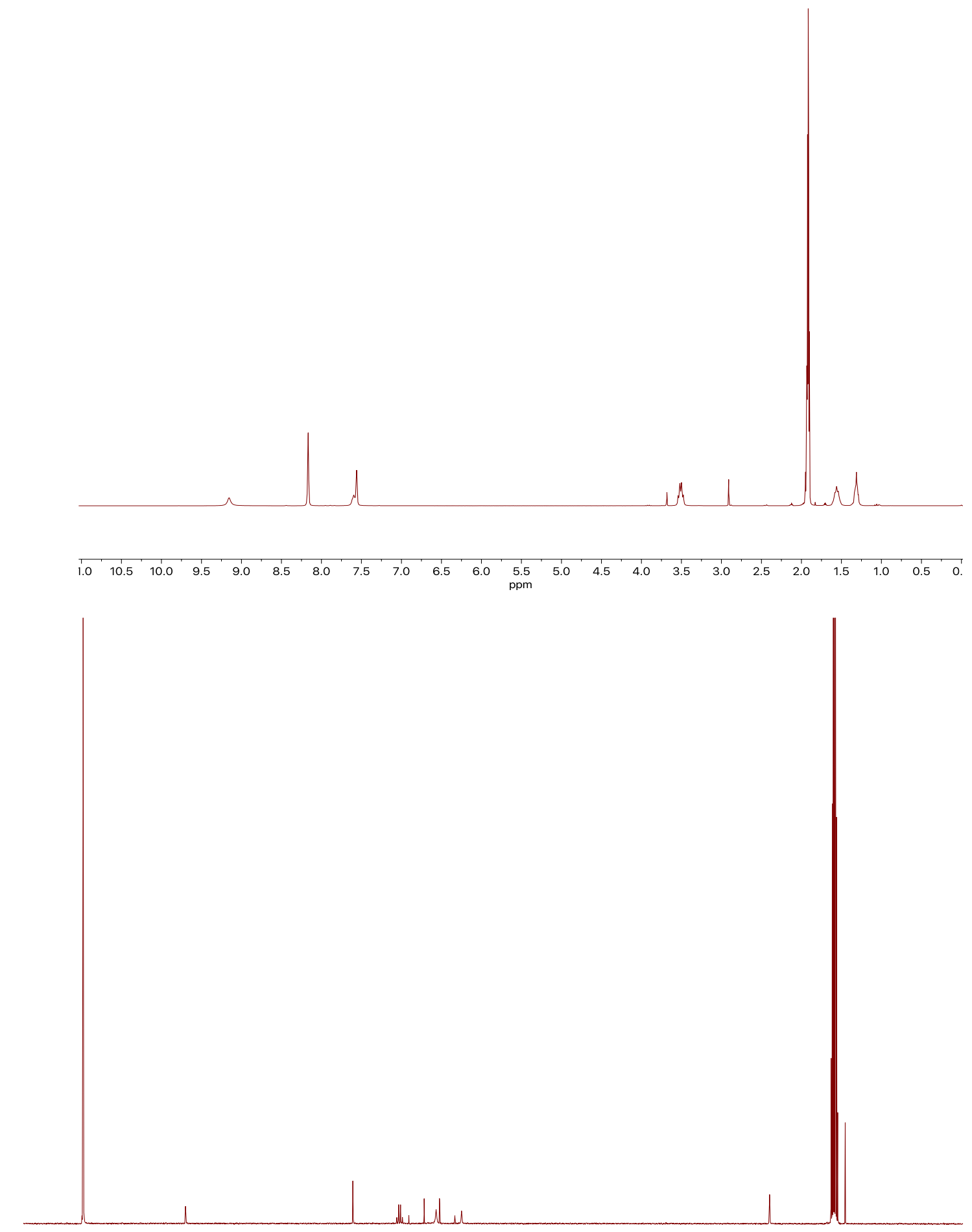

\begin{tabular}{lllllllllllllllllllllllllll}
\hline 20 & 210 & 200 & 190 & 180 & 170 & 160 & 150 & 140 & 130 & 120 & 110 & 100 & 90 & 80 & 70 & 60 & 50 & 40 & 30 & 20 & 10 & $\mathrm{C}$
\end{tabular}

Figure S26. Top) ${ }^{1} \mathrm{H}$ NMR of $6 \mathrm{CTU}$ in acetone- $d_{6}$. Bottom) ${ }^{13} \mathrm{C}$ NMR of $6 \mathrm{CTU}$ in acetone- $d_{6}$. 

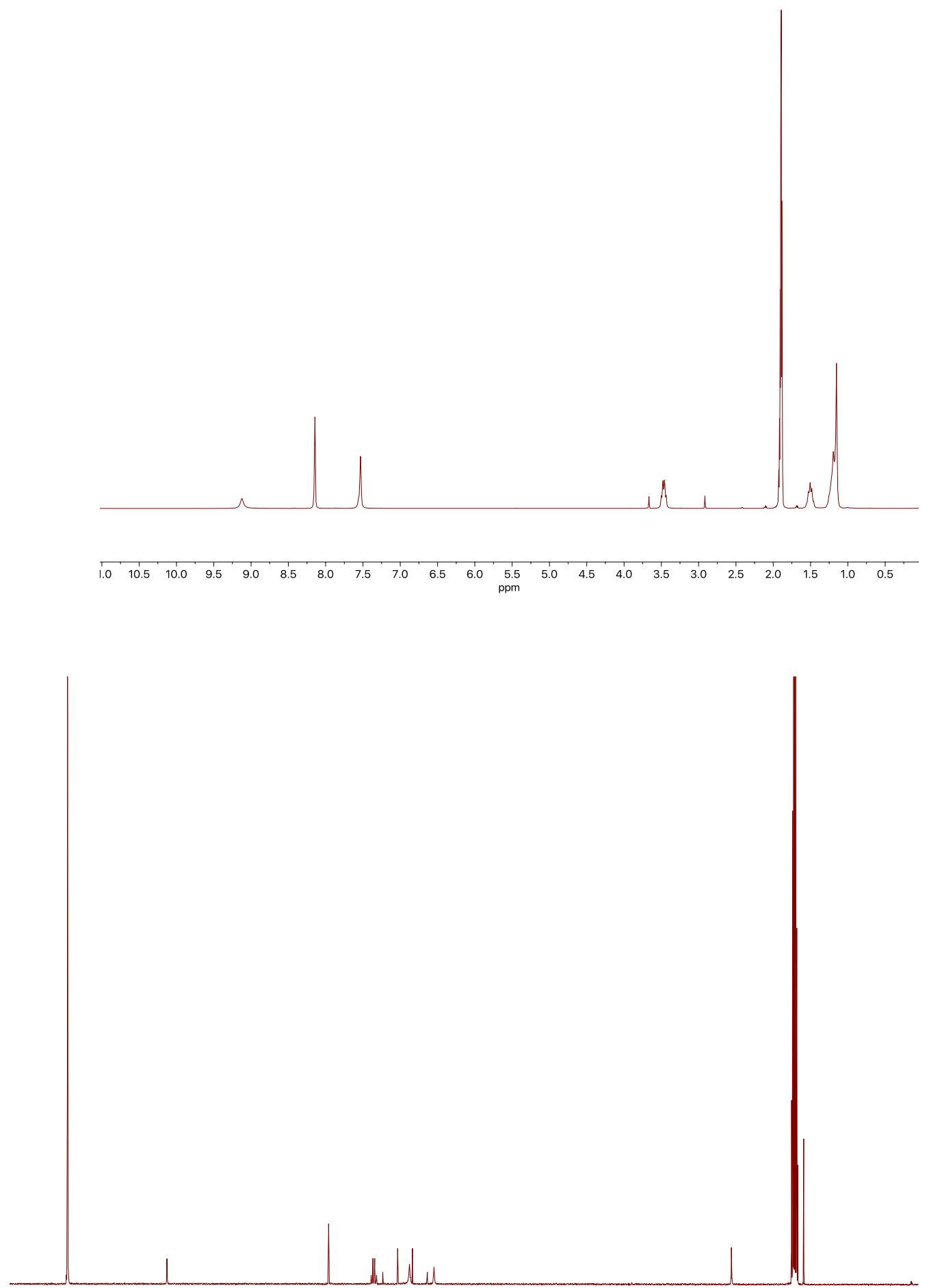

\begin{tabular}{lllllllllllllllllllllll}
\hline & 210 & 200 & 190 & 180 & 170 & 160 & 150 & 140 & 130 & 120 & 110 & 100 & 90 & 80 & 70 & 60 & 50 & 40 & 30 & 20 & 10 & $\mathrm{C}$
\end{tabular}

Figure S27. Top) ${ }^{1} \mathrm{H}$ NMR of $12 \mathrm{CTU}$ in acetone- $d_{6}$. Bottom) ${ }^{13} \mathrm{C}$ NMR of $12 \mathrm{CTU}$ in acetoned6. 


\section{CHAPTER 5}

Submitted for publication to The Journal of Chemical Education, March 2018.

Stilbene Synthesis by Olefin Metathesis Reaction

Timothy J. Bannin, Partha P. Datta, Elizabeth T. Kiesewetter and Matthew K. Kiesewetter.

Corresponding Author: Matthew Kiesewetter, Ph.D.

Chemistry

University of Rhode Island

325C, Beaupre Hall, 140 Flagg Rd.

Kingston, RI, 02881, USA

Phone: $+1-401-874-2619$

Email address: mkiesewetter@chm.uri.edu 


\section{CHAPTER 5}

\section{ABSTRACT}

In this experiment, students are asked to compare catalytic-cross metathesis and the Wittig reaction within the confines of 'Green' chemistry and atom economy. Students synthesize stilbene from styrene using Grubbs second generation catalyst. Products can be minimally characterized by IR spectroscopy and melting point but using ${ }^{1} \mathrm{H}$ NMR spectroscopy is preferred. Students find that the Wittig reaction is selective for cis-stilbene while the metathesis reaction produces $>98 \%$ trans-stilbene. Students determine the cis/trans selectivity, turnover number (TON) and maximum turnover frequency (TOF) of the reaction. The experiment is conducted alongside the synthesis of stilbene using Wittig chemistry from a published procedure. 
The 2005 Nobel Prize in Chemistry was awarded to Robert Grubbs, Yves Chauvin and Richard Schrock for the development of the metathesis reaction in organic synthesis. ${ }^{1}$ Development of metathesis chemistry continues apace, with new catalysts and abilities being reported more than a decade after the Nobel Prize. ${ }^{2}$ Indeed, the reaction has revolutionized several branches of chemistry and found applications in polymer, medicinal and organic chemistry. ${ }^{3-5}$ The olefin metathesis reaction is an intra- or inter-molecular rearrangement reaction where one or more carbon-carbon double bonds are broken and reformed. Intramolecular metathesis is generally called ring-closing metathesis, while intermolecular reactions are cross-metathesis or, sometimes, homo-cross-metathesis (homodimerization) to emphasize the use of only one reagent. Polymers can also be constructed via metathesis using acyclic diene metathesis (ADMET) or ring-opening metathesis polymerization (ROMP) methods. The process must be catalyzed, and olefin metathesis catalysts contain a metal center $r^{6,7}$ - usually $\mathrm{Ru}$ or Mo although organocatalytic methods for carbonyl-olefin metathesis have been reported. ${ }^{8}$ In an uncontrolled olefin metathesis reaction, a thermodynamic mixture of products is generated. The development of advanced (asymmetric)

$\mathrm{n}$<smiles>C=Cc1ccccc1</smiles>

$\mathrm{n}$<smiles>CC(C)=Cc1ccccc1</smiles><smiles>CCCCCCCCCCCCC</smiles>

$$
\mathrm{CH}_{2} \mathrm{Cl}_{2}
$$
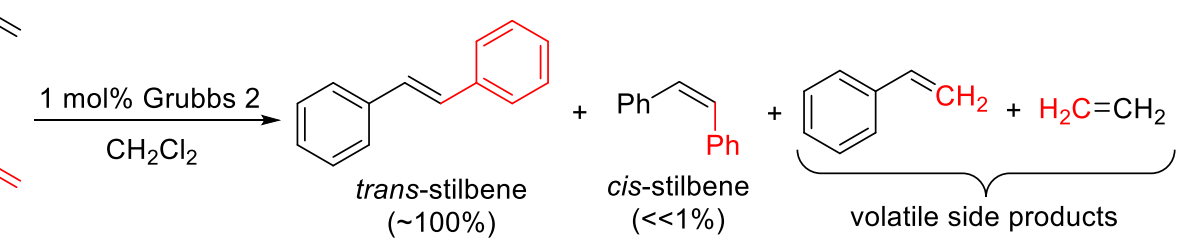

Scheme 1. The homo-cross-metathesis reaction of stilbene produces one non-volatile product. Stilbene does not reenter the catalytic cycle.

catalysts and inherent (substrate driven) kinetic or thermodynamic control often provides fewer products. In the present experiment, the sole metathesis partner, styrene, gives trans-stilbene as the only non-volatile product, Scheme 1.

The Wittig reaction, a classic means of preparing olefins, serves as a natural foil for the metathesis experiment. In the Wittig reaction, an aldehyde or ketone is reacted with a phosphonium salt in the presence of base to yield an olefin, Scheme 2. The 


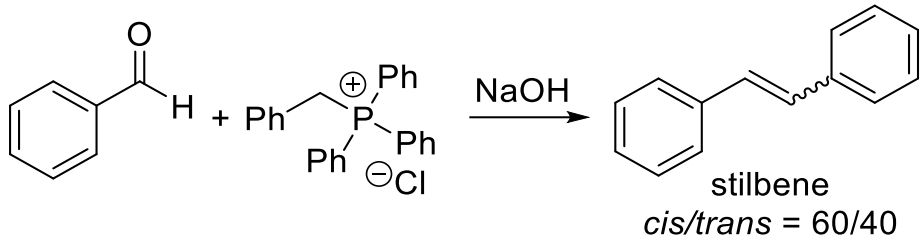

Scheme 2. Example Wittig reaction to synthesize stilbene.

Wittig reaction is a robust, widely known organic reaction that undergraduates normally learn during sophomore organic chemistry. A host of phosphonium salts is available which provides a massive diversity of alkene products. These reactions can be performed on large or small scale, are often high yielding and can easily be performed by student chemists. ${ }^{9}$ The Wittig Reaction was awarded a Nobel Prize in Chemistry. ${ }^{10}$ This reaction also is a hallmark example of a non-'Green' reaction, ${ }^{11}$ and it displays poor atom economy, ${ }^{12}$ meaning a considerable fraction of reagent mass is waste product (the triphenylphosphine oxide) which must be separated from the desired products. In contrast, metathesis catalysts are often used catalytically and can constitute a very small fraction of the reagent mass. However, the 'greenness' of a catalytic reaction often depends on the catalyst loading and metal toxicity. Metathesis catalysts are also operative in a variety of solvents and can be used heterogeneously, which facilitates catalyst removal. While the recycling of catalyst is feasible, it is often unpracticed. ${ }^{13}$ In our Advanced Organic Laboratory course, students are asked in two consecutive laboratory experiments to synthesize stilbene, first using Wittig chemistry and second by the crossmetathesis of styrene. The Wittig synthesis of stilbene, ${ }^{9}$ which reacts benzaldehyde with benzyltriphenylphosphonium chloride in the presence of base, yields predominately the cisproduct ( $60 \%$ cis-stilbene). This selectivity contrasts markedly with that of metathesis reaction, which produces $>98 \%$ trans-stilbene. This notable difference starts the students on a journey of 'unpacking' the differences, virtues and deficits of the two methods. 


\section{EXPERIMENTAL OVERVIEW}

This experiment was accomplished in an advanced organic chemistry course with 16 students. Conducting the experiment with larger numbers of students (e.g. a non-majors sophomore organic course) is feasible, but the cost of Grubbs 2 reagent should be considered. Lab sections met twice weekly for 3-hour sessions. In the lab session prior to conducting the present experiment, students synthesize stilbene in a Wittig experiment. The published Wittig procedure requires a single 3-hour lab period to complete. ${ }^{9}$ The metathesis experiment is performed over two lab sessions. On the first day, students are asked to follow a procedure to make stilbene without a partner. The metathesis experiment can easily be finished in a 3-hour lab period. On day two, students were asked to form a hypothesis and work in small groups to build a series of data to reach a conclusion. In the lab report, students are asked to compare and contrast the synthesis of stilbene with metathesis versus the Wittig reaction.

In this experiment, we employ a Ru-centered catalyst (Grubbs 2 nd generation catalyst) - (1,3Bis(2,4,6-trimethylphenyl)-2-imidazolidinylidene)dichloro(phenylmethylene)(tricyclohexylphosphine)ruthenium - which will perform the metathesis ${ }^{6}$ of styrene to make a single detectable product, trans-stilbene. ${ }^{14}$ In this transformation, the diastereoselectivity of the reaction is entirely substrate driven, producing the thermodynamic ratio of stilbene, $>98 \%$ transstilbene. 


\section{EXPERIMENTAL PROCEDURE}

Since commercial styrene contains an inhibitor from the manufacturer which may disrupt the metathesis reaction, we removed the inhibitor in bulk before the lab period began. This was achieved by stirring a mixture of $3 \mathrm{~g}$ of alumina for every $20 \mathrm{~mL}$ of styrene for 5 minutes. Then the slurry was gravimetrically filtered through a qualitative filter paper to obtain pure styrene. The students can perform the purification individually on a reduced scale. Then a $20 \mathrm{~mL}$ scintillation vial was charged with a magnetic stir bar, Grubbs $2(14.80 \mathrm{mg}, 0.017 \mathrm{mmol})$ and

dichloromethane $(10 \mathrm{~mL})$. Next, styrene $(0.2 \mathrm{~mL}, 1.74 \mathrm{mmol})$ was added to the vial. The scintillation vial was then fitted with a polymer cone or foil backed cap and placed on a stir plate to stir for about 1 hour. After 1 hour, the solvent was removed under reduced pressure.

A miniature silica column was prepared. First, a pipette $(8 \times 142 \mathrm{~mm})$ was plugged with a piece of cotton or glass wool on one end. The pipet was then filled with dry silica from $1 / 2$ to $3 / 4$ of its volume. The crude product was dissolved in about $0.5 \mathrm{~mL}$ of dichloromethane. The silica plug was then wetted with hexanes and subsequently flushed with the solution of product in hexanes. An additional $20-25 \mathrm{~mL}$ of hexanes was used to flush the product through the silica. The solvent was then removed of volatiles in vacuo and ${ }^{1} \mathrm{H}-\mathrm{NMR}$, IR and a melting point were obtained. Students use chemical shift in the ${ }^{1} \mathrm{H}$ NMR spectrum to identify cis- versus transstilbene, but melting point can also be used to identify which diastereomer is made. 


\section{HAZARDS}

All synthesized products and intermediates should be handled with caution. Appropriate personal protective equipment should be used at all times, and the reagents should only be handled in a well-ventilated fume hood. Avoid contact with skin, and in the event of accidental exposure, wash the afflicted area with copious amounts of water. Styrene is flammable, may cause skin irritation, is a serious eye irritant, a suspected carcinogen and suspected of damaging fertility or the unborn child. Grubbs 2 is a flammable solid. $\mathrm{CH}_{2} \mathrm{Cl}_{2}$ can cause skin irritation, is a serious eye irritant, may cause respiratory irritation, may cause drowsiness/dizziness, suspected of causing cancer, if swallowed it may cause damage to the liver, blood and if inhaled it may cause damage to the central nervous system. Hexanes is highly flammable, may be fatal if swallowed and enters the airways, can cause skin irritation, may cause drowsiness/dizziness, is suspected of damaging fertility or the unborn child, may cause damage to the nervous system and is toxic to aquatic life. $\mathrm{CDCl}_{3}$ is harmful if swallowed, causes skin and serious eye irritation, toxic if inhaled, suspected of causing cancer and of damaging fertility or the unborn child and can cause damage to organs. 


\section{RESULTS AND DISCUSSION}

This laboratory experiment was designed with two goals in mind: to give students experience with popular and versatile metathesis chemistry and to contrast this chemistry with the complementary Wittig reaction, which students performed previously in the semester from a published procedure. ${ }^{9}$ Students observe several differences in the two reactions. The Wittig produces mostly cis-stilbene while metathesis makes trans-stilbene; the Wittig requires stoichiometric reagents while metathesis is catalytic; both reactions require purification to remove catalyst or phosphine oxide, but different methods of purification are required. This experiment also employs common and advanced organic chemistry concepts and techniques that students will find useful in industrial or academic setting: rotary evaporation, filtration, flash chromatography on small scale, spectroscopic identification, thermodynamic versus kinetic selectivity, properties of diastereomers and catalysis.

The purification of the reaction is facile. Students generally obtained $80-90 \%$ yield after the column chromatography purification. Because the reaction is highly selective, melting point can also be used to identify the isomer (m.p. cis-stilbene $=-5^{\circ} \mathrm{C}$, m.p. trans-stilbene $=122-126$ $\left.{ }^{\circ} \mathrm{C}\right),{ }^{15,16}$ and students find values of $120-124^{\circ} \mathrm{C}$. This and IR spectroscopy provide reasonable

proof of compound identity and purity; however, we asked students to use ${ }^{1} \mathrm{H}$ NMR spectroscopy to identify the product. The chemical literature indicates that the olefinic resonances for transstilbene $(7.15 \mathrm{ppm})$ appear markedly downfield of those for cis-stilbene $(6.57 \mathrm{ppm})$ in the ${ }^{1} \mathrm{H}$ NMR spectrum. ${ }^{17}$ Further, close examination of the 6.1-8.0 ppm region of the spectrum reveals no spectroscopic indication of cis-product, indicating nearly perfect diastereoselectivity. The reaction is under thermodynamic control and produces a minor amount $(0.2 \%)$ of the cis-isomer, but this small amount cannot be detected by ${ }^{1} \mathrm{H}$ NMR or melting point analysis. We were not equipped in our lab; however, HPLC or GC could be performed to detect cis-stilbene; a very small amount is expected to be present.

On the second day of experimentation, students are asked to form a hypothesis and work in teams to come to a conclusion. Common variations included testing the turnover number and frequency limits of the reaction (within a lab period). These values are bookended by raising 
and lowering the catalyst concentration and conducting the workup (quenching the reaction) at various time points. Students measured turnover numbers (TON) of TON $=67-92$ and turnover frequency of TOF $=0.96-1.5 \mathrm{~min}^{-1}$. Students were also able to construct a crude first order plot (styrene) by quenching identical reactions at different time points and determining conversion by ${ }^{1} \mathrm{H}$ NMR. Quenching the reaction at various time points allowed some students to observe that the cis/trans ratio does not change as a function of conversion. From this, they concluded that the reaction was under thermodynamic control. Some students asked if the stabilizer slows down the reaction; the students were not able to discern a difference in TON or TOF with or without stabilizer in the styrene.

Students were graded based on the purity of their product (NMR and melting point) in addition to the post lab questions. The main objective the questions is to get the students to compare Wittig and metathesis methodologies. The obvious difference in cis/trans ratios between the methods was universally identified. After literature searching (or conducting cis/trans ratio versus reaction time experiments), most students identified that the metathesis reaction was under thermodynamic control and the Wittig exhibits a kinetic preference for the cis- isomer. 


\section{CONCLUSION}

At its core, this laboratory experiment is not about stilbene or metathesis, but rather it is about introducing the students to the unclear nature behind the concepts of Green chemistry ${ }^{18}$ and atom economy ${ }^{12}$ by comparing two robust and complementary synthetic approaches. Students were able to understand the concept of atom economy by stating that the metathesis reaction produced less reagent waste product than the Wittig. However, some students insist the Wittig is more utilitarian due to the facile nature of separation in that lab experiment. To us, there is no clear answer as to which process is 'Greener' or less wasteful (atom economic plus purification waste), but some students were able to present nuanced arguments for both sides. We feel that being able to see the big picture - even if it does not contain any clear answer(s) - is a primary goal of comparing these two reactions. 


\section{Acknowledgments}

This work has been supported by an NSF CAREER Award (CHE 1554830) and the University of Rhode Island. P.P.D. would like to thank the University of Rhode Island Graduate School for a graduate fellowship. 


\section{LIST OF REFERENCES}

1 Grubbs, R. H. Olefin-Metathesis Catalysts for the Preparation of Molecules and Materials (Nobel Lecture). Angew. Chem. Int. Ed. 2006, 45 (23), 3760-3765.

2 Montgomery, T. P.; Johns, A. M.; Grubbs, R. H. Recent Advancements in Stereoselective Olefin Metathesis Using Ruthenium Catalysts. Catalysts. 2017, 7 (3), 87.

3 Sinclair, F.; Alkattan, M.; Prunet, J.; Shaver, M. P. Olefin Cross Metathesis and RingClosing Metathesis in Polymer Chemistry. Polym. Chem. 2017, 8 (22), 3385-3398.

4 Liu, M.; Mountford, S. J.; Richardson, R. R.; Groenen, M.; Holliday, N. D.; Thompson, P. E. Optically Pure, Structural, and Fluorescent Analogues of a Dimeric $Y_{4}$ Receptor Agonist Derived by an Olefin Metathesis Approach. J. Med. Chem. 2016, 59 (13), 60596069.

5 Grandner, J. M.; Shao, H.; Grubbs, R. H.; Liu, P.; Houk, K. N. Origins of the Stereoselective Mechanism of Olefin Metathesis with Ru-Dithiolate Catalysts. J. Org. Chem. 2017, 82 (19), 10595-10600.

6 Chatterjee, A. K.; Choi, T. -L.; Sanders, D. P.; Grubbs, R. H. A General Model for Selectivity in Olefin Cross Metathesis. J. Am. Chem. Soc. 2003, 125 (37), 11360-11370.

7 Nguyen, T.; Koh, M. J.; Shen, X.; Romiti, F.; Schrock, R. R.; Hoveyda, A. H. Kinetically Controlled E-selective Catalytic Olefin Metathesis. Science, 2016, 352 (6285), 569-575.

8 Griffith, A. K.; Vanos, C. M.; Lambert, T. H. Organocatalytic Carbonyl-Olefin Metathesis. J. Am. Chem. Soc. 2012, 134 (45), 18581-18584.

9 Warner, J. C.; Anastas, P. T.; Anselme, J.-P. The Wittig Reaction in the Undergraduate Organic Laboratory. J. Chem. Educ. 1985, 62 (4), 346.

10 Gericke, D. Nobel Prize for Chemistry 1979 for the Wittig Reaction as a Basis for ManySided Syntheses. Georg Wittig, 60 ${ }^{\text {th }}$ German Nobel Laureate. Fortschr. Med. 1979, 97 (43), 1958-1964.

11 Noyori, R. Pursuing Practical Elegance in Chemical Synthesis. Chem. Commun. 2005, $14,1807-1811$. 
12 Trost, B. M. Atom Economy - A Challenge for Organic Synthesis: Homogeneous Catalysis Leads the Way. Angew. Chem. Int. Ed. Engl. 1995, 34 (3), 259-281.

13 Lwin, S.; Wachs, I. E. Olefin Metathesis by Supported Metal Oxide Catalysts. ACS Catal. 2014, 4 (8), 2505-2520.

14 Sabila, P. Styrene Cross-Metathesis Using Low Catalyst Concentrations. Univers. J. Chem. 2015, 3 (3), 87-90.

15 Thermo Fisher Scientific and Acros Organics. Cis-Stilbene, (97\%); Safety Data Sheet; Fair Lawn, NJ, 2018.

16 Thermo Fisher Scientific and Acros Organics. Trans-Stilbene; Safety Data Sheet; Fair Lawn, NJ, 2018.

17 Wheeler, O. H.; Battle de Pabon, H. N. Synthesis of Stilbenes. A Comparative Study. J. Org. Chem. 1965, 30 (5), 1473-1477.

18 Doxsee, K. M.; Hutchison, J. E. Green Organic Chemistry: Strategies, Tools, and Laboratory Experiments; Thomson Learning Custom Publishing, 2002. 
SUPPORTING INFORMATION FOR: Stilbene Synthesis by Olefin Metathesis Reaction

TABLE OF CONTENTS

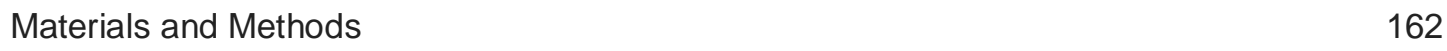

$\begin{array}{ll}\text { Special Hazards } & 165\end{array}$

$\begin{array}{ll}\text { Notes to Instructors } & 166\end{array}$

Example Post Lab Questions and Answers 168

Characterization Data and Spectra of Stilbene Products 172

$\begin{array}{ll}\text { References for Supplemental Information } & 176\end{array}$

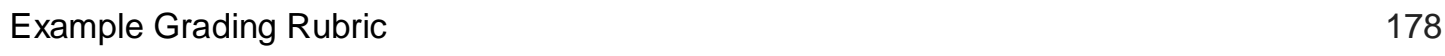

$\begin{array}{lr}\text { Student Handout } & 180\end{array}$ 


\section{Materials and Methods}

Styrene was purchased from Acros Organics, Grubbs $2^{\text {nd }}$ generation catalyst from SigmaAldrich, ACS reagent grade dichloromethane and hexanes from Fisher Scientific. Silica gel (60Å/200-425 mesh) was purchased from Silicycle. $\mathrm{CDCl}_{3}$ was purchased from Cambridge Isotope Laboratories. NMR experiments were performed on a Bruker Avance III $300 \mathrm{MHz}$ spectrometer in $\mathrm{CDCl}_{3}$. IR spectra were obtained on a Thermo Nicolet $380 \mathrm{FT}$-IR equipped with a Smart Orbit attachment. Melting points were obtained on a Stuart SMP10 melting point apparatus.

Required Reagents (CAS number)

1. (1,3-Bis(2,4,6-trimethylphenyl)-2-

imidazolidinylidene)dichloro(phenylmethylene)(tricyclohexyl-phosphine)ruthenium(II) (Grubbs Catalyst $2^{\text {nd }}$ Generation, CAS 246047-72-3)

2. styrene (CAS 100-42-5)

3. dichloromethane (CAS 75-09-2)

4. hexanes (CAS 110-54-3)

5. silica gel (60尺̊/200-425 mesh, CAS 7631-86-9)

6. $\mathrm{CDCl}_{3}(\mathrm{CAS} 865-49-6)$

Apparatus and Lab Materials

Students will each require:

1. $20 \mathrm{~mL}$ scintillation vial with a polypropylene screw cap

2. magnetic stir bar $(0.5 \times 0.125$ in. $)$

3. glass wool

4. volumetric pipette bulb

5. $8 \times 142 \mathrm{~mm}$ glass pipettes

6. 9-inch Pasteur pipettes

7. 3 cc pipette bulb 
8. 3-pronged clamp

9. clamp stand with base

10. $3 \times 3$ inch weighing paper (for loading silica into pipette)

11. vial-to-rotavap adapter (we use a 24/40 septa, 1 - 1 1/4 in 22 G needle)

12. magnetic stir plate

Students need access to shared:

1. Rotary evaporator

2. IR spectrometer

3. Melting point apparatus

4. (optional) ${ }^{1} \mathrm{H}$ NMR spectrometer 


\section{Design of Experiment}

This experiment can fill one or two 3-hour lab periods. The main experiment, designed to take one day, takes the student through the synthesis of stilbene. The second day is freeform, and the students are encouraged to pair with one or more students to gather additional information about the reaction.

Common kinetics-type experiments on the second day include: Finding the order of the reaction in a reagent by collecting conversion versus time data (the order in Grubbs 2 requires at least two observed rate constants ( $\left.\mathrm{k}_{\mathrm{obs}}\right)$ from the first order plot of [styrene] vs time), determining the turnover number and limits thereof for the reaction. The reaction is first order in [Grubbs 2]o and first order in [styrene].

Other experiments include varying the reagents. Students can also attempt the reaction with Grubbs catalyst, $1^{\text {st }}$ Generation (Grubbs 1), but this catalyst produces no conversion even at high catalyst loadings. This is related to the olefin type. ${ }^{1,2}$ Students can also run the reaction in the presence of inhibitor; no change in the reaction versus the uninhibited reaction is observed. 


\section{Special Hazards}

All synthesized products and intermediates should be handled with caution. Avoid contact with skin and in the event of accidental exposure, wash the afflicted area with copious amounts of water. Styrene is flammable, may cause skin irritation, is a serious eye irritant, a suspected carcinogen and suspected of damaging fertility or the unborn child. Grubbs 2 is a flammable solid. $\mathrm{CH}_{2} \mathrm{Cl}_{2}$ can cause skin irritation, is a serious eye irritant, may cause respiratory irritation, may cause drowsiness/dizziness, suspected of causing cancer, if swallowed it may cause damage to the liver, blood and if inhaled it may cause damage to the central nervous system. Hexanes is highly flammable, may be fatal if swallowed and enters the airways, can cause skin irritation, may cause drowsiness/dizziness, is suspected of damaging fertility or the unborn child, may cause damage to the nervous system and is toxic to aquatic life. $\mathrm{CDCl}_{3}$ is harmful if swallowed, causes skin and serious eye irritation, toxic if inhaled, suspected of causing cancer and of damaging fertility or the unborn child and can cause damage to organs. Appropriate personal protective equipment should be used at all times, and the reagents should only be handled in a well-ventilated fume hood. 
Notes to Instructors

Removal of Inhibitor. Styrene from a chemical supplier contains an inhibitor. The inhibitor may not be disruptive to the metathesis reaction, but it was removed prior to the lab period by stirring a mixture of $3 \mathrm{~g}$ of alumina for every $20 \mathrm{~mL}$ of styrene for $5 \mathrm{~min}$. Then, the slurry was filtered gravimetrically through qualitative filter paper. Uninhibited styrene will undergo auto polymerization over several days; this inhibitor-free styrene should be disposed of after the lab period, and the glassware cleaned.

Solvent Removal from a Vial by Rotovap. To remove solvent by rotovap from a $20 \mathrm{~mL}$ scintillation vial requires a specialized adapter (Chemglass CG-1318-10 Glass Rotary Evaporator Vial Adapter, 24/40 Joint). However, we employ 24/40 septa and needles which are usually readily available in an organic chemistry lab. To attach the vial to the rotovap, the septa must be inverted so the opening of the vial fits into the $40 \mathrm{~mm}$ side of the septa. Then, insert the needle through the $24 \mathrm{~mm}$ side which fits as a slip joint on a 14/20 bump trap or 14/20 adapter.

Metathesis Reaction. Our students ran reactions in disposable $20 \mathrm{~mL}$ scintillation vials, but a conventional $10 \mathrm{~mL}$ round bottom flask is acceptable. The students should notice a dark purple color upon the addition of the Grubbs 2 catalyst. The Grubbs 2 catalyst can be dispensed in a stock solution of $\mathrm{CH}_{2} \mathrm{Cl}_{2}$, but this stock solution has a finite lifetime. Students were asked to syringe styrene directly from the dispensing area (in a hood) and transport the capped syringe back to their workspace. This greatly minimized exposure to styrene, which has a potent odor. Purification by Silica Gel Chromatography. Our students purified their stilbene with a microscale, Pasteur pipette silica gel column. A glass wool/cotton plug was loaded into the column ( $8 \times 142 \mathrm{~mm}$ glass pipette) using a 9 inch Pasteur pipette push rod, see Figure $1 \mathrm{~A}$ in the student handout section. Then silica was loaded into the $8 \times 142 \mathrm{~mm}$ pipette using weigh paper folded in half diagonally. A volumetric pipette bulb was used to force hexanes through the column with slight, constant pressure. The silica bed can crack if abrupt pressure changes are applied. A traditional silica gel column can also be employed, but once practiced, we find that the pipette column becomes a favorite tool for easy separations. Students achieved the 
best and most facile separations when the product was loaded onto the column in a minimal volume of $\mathrm{CH}_{2} \mathrm{Cl}_{2}(<0.5 \mathrm{~mL})$ and eluted with hexanes $(\sim 25 \mathrm{~mL})$. Students should be reminded to load the product solution entirely onto the silica before eluting with hexanes. Our students typically get an isolated yield of $\sim 70-90 \%$. Students who do not obtain a yield of at least $60 \%$ may be able to flush their column with more hexanes to obtain residual product on the silica gel. Identification of cis- versus trans-stilbene. Students will observe that the metathesis reaction produces $\sim 100 \%$ trans-stilbene. The cis/trans ratio is most conveniently determined from ${ }^{1} \mathrm{H}$ NMR, where the chemical shift of the ethylene resonance is isomer-dependent: cis-stilbene at $6.6 \mathrm{ppm}$ and trans-stilbene at $7.15 \mathrm{ppm}^{3}$ With Grubbs 2, the metathesis reaction should produce the approximate thermodynamic ratio of products. For stilbene, the thermodynamic ratio is $\sim 0.2 \%$ cis-isomer, $\mathrm{K}_{\text {eq }}=0.002, \square \mathrm{G}^{\circ}=3.7 \mathrm{kcal} / \mathrm{mol}$. In our experimentation, we do not observe any cis-stilbene in the ${ }^{1} \mathrm{H}$ NMR spectrum. Alternatively, the melting points of the two isomers are drastically different (cis- m.p. $=-5^{\circ} \mathrm{C}$ and trans- m.p. $\left.=122-126^{\circ} \mathrm{C}\right) .^{4,5}$ Potential post-lab questions are apparent: 


\section{Example Post Lab Questions and Answers}

We select 4-5 of the questions below as post lab questions to be answered in the lab report.

1. What are the advantages of synthesizing stilbene with metathesis vs Wittig? Disadvantages?

Answer: Wittig processes confer high selectivity for the cis-isomer, which can be difficult to access using metathesis. Wittig reagents and methods are robust, structurally diverse and are often easy to separate from the product, but they must be used stoichiometrically. Metathesis catalysts are highly functional group tolerant, readily available and general (i.e. one can apply a SINGLE metathesis catalyst to many syntheses, but a new Wittig reagent is needed for every product). The Grubbs reagents (we use the common term 'catalyst' in this document are really pre-catalysts or initiators) ${ }^{1}$ are usually applied catalytically which minimized waste. However, the metathesis products can re-enter the catalytic cycle, eroding yield and stereocontrol (if present), depending on what type of olefin describe the product and reagent. ${ }^{1}$ Stilbene is a Type II olefin with respect to Grubbs $1^{\text {st }}$ generation catalyst, ${ }^{1}$ and it will not readily undergo subsequent metathesis. However, if the product is symmetric (as with stilbene), these processes are not evident even if they occur.

2. What is the cis/trans ratio produced by metathesis and how does it compare to the Wittig reaction?

Answer: According to Warner et al., the Wittig reaction produced cis- and trans-stilbene in a $60: 40$ ratio while the present metathesis reaction produces $\sim 100 \%$ trans- product. $^{6}$ The Wittig reaction is selective for cis-product while Grubbs 2 is not selective.

3. Draw the catalytic cycle that produces stilbene. Where is the stereochemistry set (i.e. at what point does the product become cis- or trans-? 

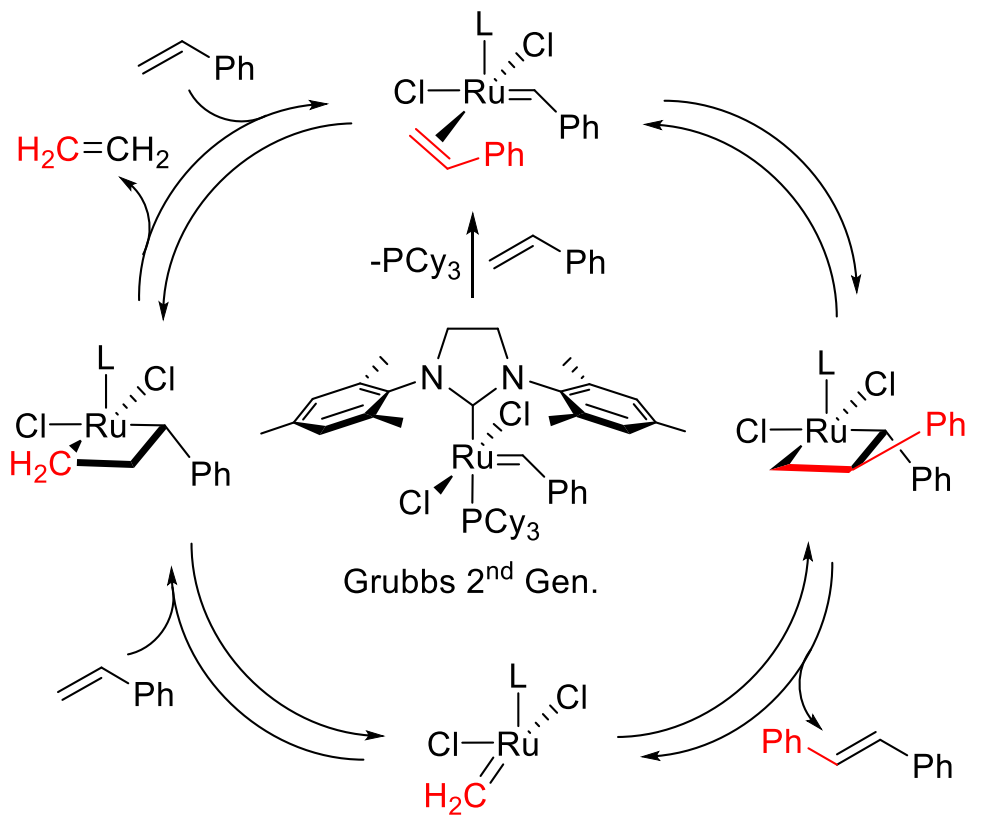

Answer: A full answer will include the catalytic cycle above which shows the generation of the active catalyst (middle to top), formation of the ruthenium metallocycle butane (right) where the stereochemistry of the product is set, and the regeneration of the catalytically productive ruthenium benzylidene (top) via evolution of an equivalent of ethylene (left).

4. A properly-designed catalyst can produce non-thermodynamic distributions of products (i.e. a kinetic or Curtin-Hammett distribution of products). Is this metathesis reaction thermodynamically or kinetically controlled? Can you design an experiment to test your answer?

Answer: The product distribution in the present metathesis reaction is under thermodynamic control; the achiral catalyst imparts no kinetic preference for one isomer over another. However, asymmetric metathesis catalysts are available.

To test the possibility of thermodynamic versus kinetic control, a group of students can perform reactions where the reactions are stopped at different intervals: from 20 min up to days. Students will observe only trans-product at all time points. If the reaction were under 
measurable kinetic control, cis/trans ratio would be a function of reaction time. This requires stilbene to undergo metathesis, which as a Type II olefin, ${ }^{1}$ it does so only sparingly.

5. Is this catalyst a good choice for olefin metathesis? (hint: take a look at your TON and TOF). Hit the literature, what other catalyst might you suggest for metathesis?

Answer: Grubbs-type catalysts are widely used because they are long-lived (decent TON) and tolerant to a wide variety of functional groups and reaction conditions. ${ }^{1,7}$ A host of metathesis catalysts is available. Various specialized catalysts are available for rapid initiation, ${ }^{8}$ ringclosing metathesis, ${ }^{9}$ and densely-functionalized substrates. ${ }^{10}$ Catalysts employing other metals, particularly molybdenum, are capable of effecting rapid and selective metathesis reactions. ${ }^{11}$

6. If you produced the thermodynamic ratio of stilbene (trans-stilbene $\Leftrightarrow$ cis-stilbene; $\mathrm{K}_{\mathrm{eq}}$ $=0.002)$, why is none observed in the ${ }^{1} \mathrm{H}$ NMR?

Answer: The thermodynamic ratio suggests $0.2 \%$ cis- product $\left(K_{\text {eq }}=0.002=(100-x) / x ; x=99.8\right)$. This value is far below the detection limits of NMR spectroscopy.

\section{What factors influence cis/trans ratios?}

Answer: Catalysts and reagents (e.g. Wittig) can be stereoselective, but the Grubbs 2-catalyzed formation of stilbene from styrene is not. This experiment produces the thermodynamic ratio of products. This ratio is determined by the relative stability of the two products where the bulky phenyl rings strongly favor a trans-isomer for steric reasons. ${ }^{1}$

8. Why is the cis/trans ratio of stilbene so small? For comparison, the thermodynamic distribution of isomers for 2-butene is about $30 \%$ cis-isomer. ${ }^{12}$ 
Answer: The phenyl rings in stilbene are much bulkier than the methyl groups in 2-butene, which makes the reaction far more selective for the trans-product in the case of stilbene versus 2-butene. The effect is augmented because the phenyl rings in stilbene prefer to be coplanar for $\pi$-delocalization.

9. Convert cis/trans ratio into $\mathrm{K}_{\mathrm{eq}}$ and/or $\Delta \mathrm{G}^{\circ}$.

Answer: The values are $\sim 0.2 \%$ cis-isomer, $\mathrm{K}_{\mathrm{eq}}=0.002, \Delta \mathrm{G}^{\circ}=3.7 \mathrm{kcal} / \mathrm{mol}$. This can be found using the standard equations:

$$
\begin{gathered}
K_{e q}=\frac{[\text { trans }- \text { stilbene }]_{e q}}{[\text { cis }- \text { stilbene }]_{e q}} \\
\Delta G^{0}=-R T \ln K_{e q}
\end{gathered}
$$


Characterization Data and Spectra of Stilbene Products

trans-stilbene

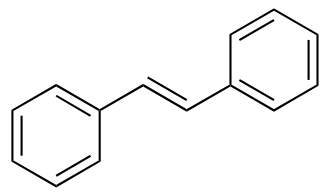

${ }^{1} \mathrm{H}-\mathrm{NMR}\left(300 \mathrm{MHz}, \mathrm{CDCl}_{3}\right): \delta 7.57-7.47$ (d, $\left.J=7.6 \mathrm{~Hz}, 2 \mathrm{H}\right), 7.43-7.32(\mathrm{t}, J=14.8,7.9,7.0$ $\mathrm{Hz}, 2 \mathrm{H}), 7.32-7.21(\mathrm{t}, J=14.8,8.5,6.0 \mathrm{~Hz}, 1 \mathrm{H}), 7.15-7.09(\mathrm{~s}, 1 \mathrm{H})$.

IR: $A=3058.69 \mathrm{~cm}^{-1}(=\mathrm{C}-\mathrm{H}) ; \mathrm{B}=3020.13 \mathrm{~cm}^{-1}(=\mathrm{C}-\mathrm{H}) ; \mathrm{C}=1596.85 \mathrm{~cm}^{-1}, 1577.56 \mathrm{~cm}^{-1}(\mathrm{C}=\mathrm{C}$, aromatic); $\mathrm{D}=1494.64 \mathrm{~cm}^{-1}\left(\mathrm{C}=\mathrm{C}\right.$, aromatic); $\mathrm{E}=1450.28 \mathrm{~cm}^{-1}(\mathrm{C}=\mathrm{C}$, aromatic) (see spectra below)

MP: $122-125^{\circ} \mathrm{C}$

Yield: About 92\% (determined by NMR).

cis-stilbene (for comparison, none observed)<smiles>C(=C\c1ccccc1)\c1ccccc1</smiles>

${ }^{1} \mathrm{H}$ NMR $\left(89.56 \mathrm{MHz}, \mathrm{CDCl}_{3}\right):{ }^{13} \delta 7.38-6.98(\mathrm{~m}, 10 \mathrm{H}), 6.57(\mathrm{~s}, 2 \mathrm{H})$

$M P: 4-5^{\circ} \mathrm{C}$ 


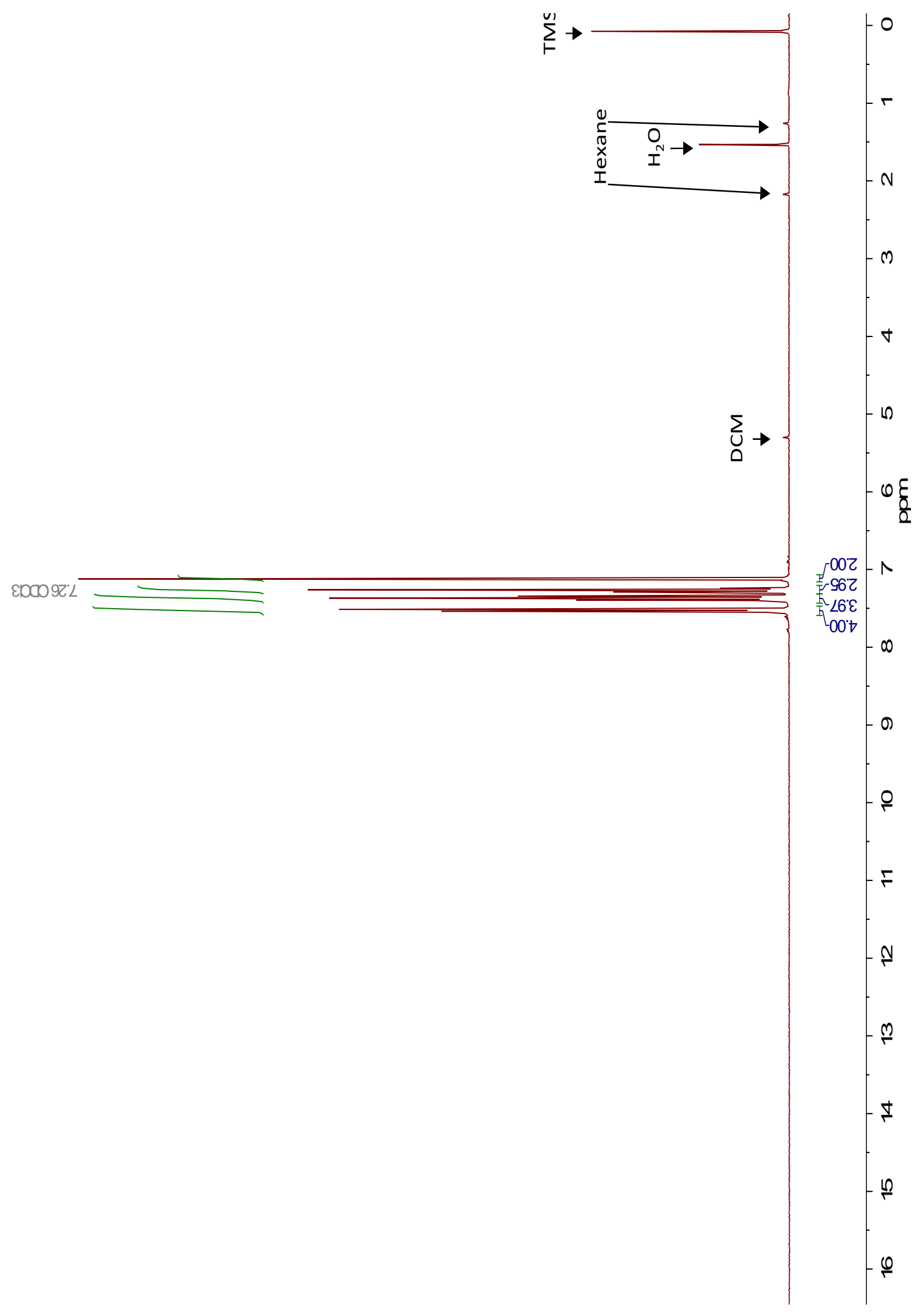




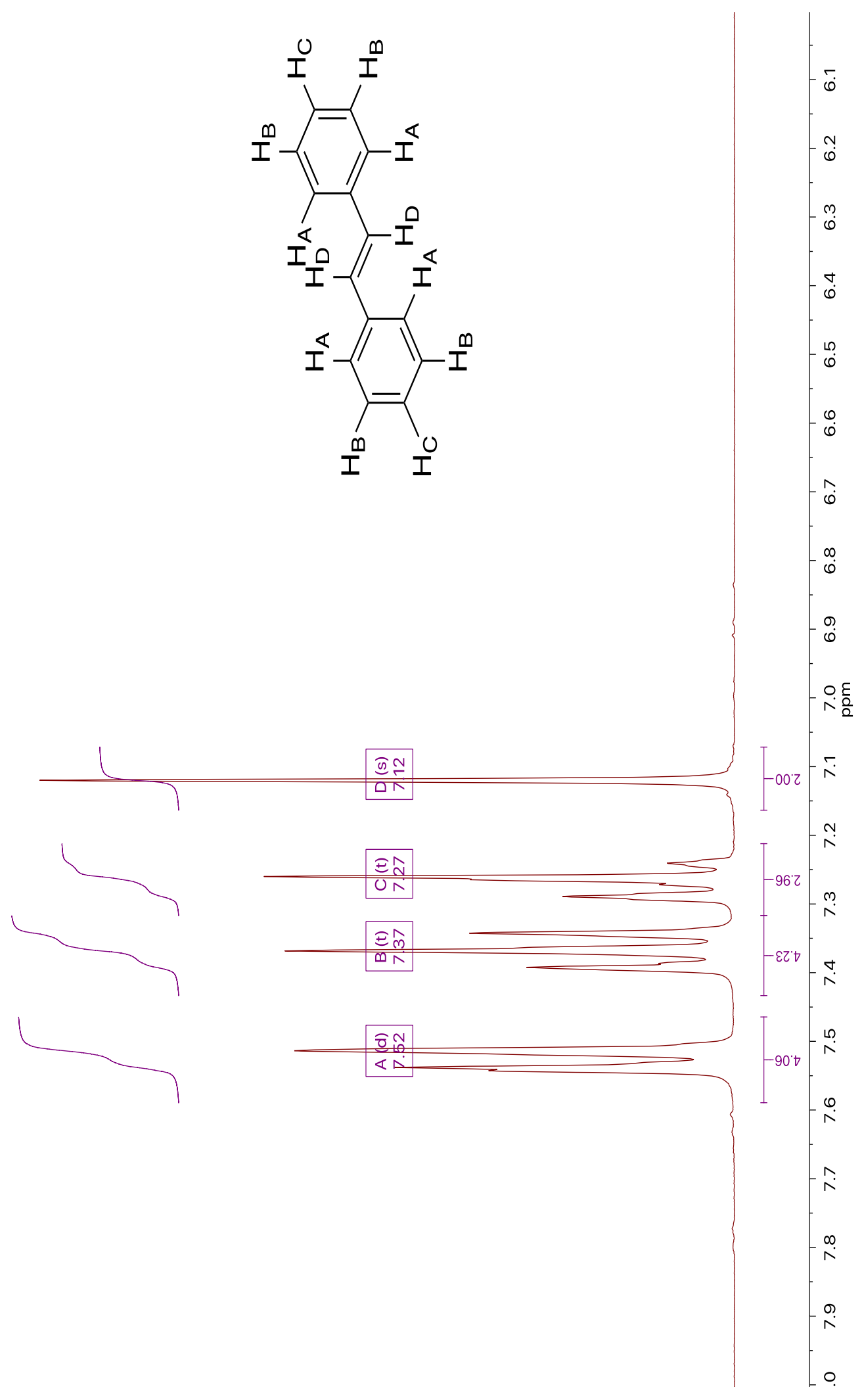




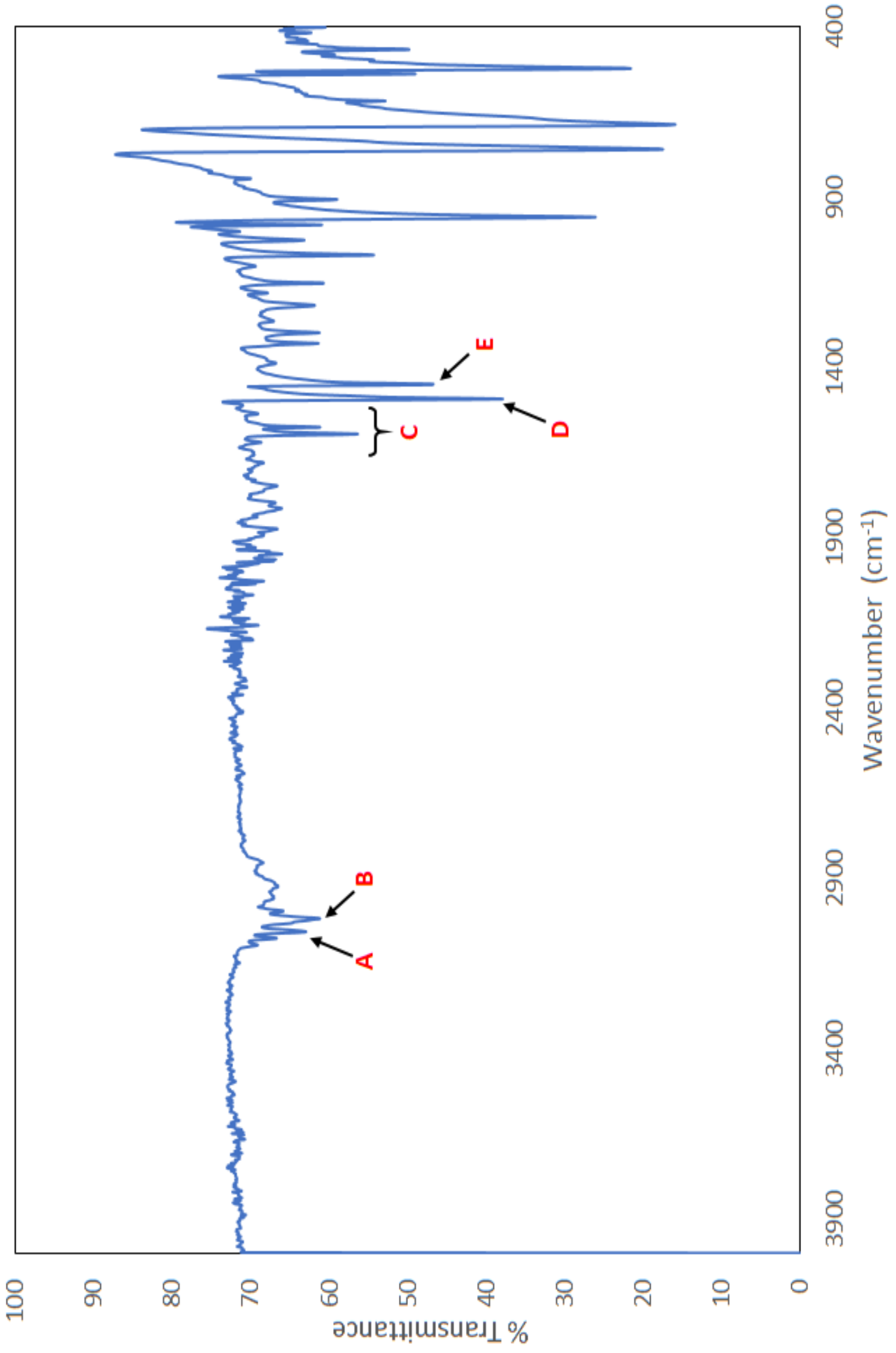


List of References for Supplemental Information

1. Chatterjee, A. K.; Choi, T. -L.; Sanders, D. P.; Grubbs, R. H. A General Model for Selectivity in Olefin Cross Metathesis. J. Am. Chem. Soc. 2003, 125 (37), 11360-11370.

2. Sabila, P. Styrene Cross-metathesis Using Low Catalyst Concentrations. Univers. J. Chem. 2015, 3 (3), 87-90.

3. Wheeler, O. H. Battle de Pabon, H. N. Synthesis of Stilbenes. A Comparative Study. J. Org. Chem. 1965, 30 (5), 1473-1477.

4. Fisher Scientific and Acros Organics. Cis-Stilbene, (97\%); Safety Data Sheet; Fair Lawn, NJ, 2018.

5. Fisher Scientific and Acros Organics. Trans-Stilbene, (97\%); Safety Data Sheet; Fair Lawn, NJ, 2018.

6. Warner, J. C.; Anastas, P. T.; Anselme, J. -P. The Wittig Reaction in the Undergraduate Organic Laboratory. J. Chem. Ed. 1985, 62 (4), 346.

7. Dinger, M. B.; Mol, J. C. High Turnover Numbers with Ruthenium-Based Metathesis Catalysts. Adv. Synth. Catal. 2002, 344 (6-7), 671-677.

8. Romero, P. E.; Piers, W. E.; McDonald, R. Rapidly Initiating Ruthenium OlefinMetathesis

Catalysts. Angew. Chem. 2004, 116 (45), 6287-6291.

9. Nguyen, S. T.; Grubbs, R. H. Syntheses and Activities of New Single-Component, Ruthenium-Based Olefin Metathesis Catalysts. J. Am. Chem. Soc. 1993, 115 (21), 9858-9859.

10. Stewart, I. C.; Ung, T.; Pletnev, A. A.; Berlin, J. M.; Grubbs, R. H.; Schrodi, Y. Highly Efficient Ruthenium Catalysts for the Formation of Tetrasubstituted Olefins via RingClosing Metathesis. Org. Lett. 2007, 9 (8), 1589-1592.

11. Nguyen, T.; Koh, M. J.; Shen, X.; Romiti, F.; Schrock, R. R.; Hoveyda, A. H. Kinetically controlled E-selective catalytic olefin metathesis. Science, 2016, 352 (6285), 569-575

12. Scott, R. B.; Ferguson, W. J.; Brickwedde, F. G. Thermodynamic Properties of cis-2Butene from $15^{\circ}$ to $1500^{\circ}$ K. J. Res. Natl. Bur. Stand. 1944, 33 (1), 1-20. 
13. Spectral Database for Organic Compounds (SDBS). SDBS- ${ }^{-1} \mathrm{H}$ NMRSDBS No. 7295HSP-04-012 cis-stilbene.

http://sdbs.db.aist.go.jp/sdbs/cgibin/direct frame top.cgi (accessed Jan 25, 2018). 
Example Grading Rubric

1. Descriptive Title/Purpose (5 pts): Title should be concise yet describe the experiment completely. A sentence or two should be devoted to the purpose of the experiment.

2. Reaction Scheme (10 pts): A reaction scheme should be provided after the title and purpose. The scheme should pertain to this specific experiment including reagents, reaction conditions and product. A complete mechanism for the reaction should follow with proper arrow pushing and formal charges.

3. Data Analysis and Characterization (20 pts): All the spectra should be provided, including IR and NMR data. These should be properly labeled with assignments of relevant peaks. A table or lists of peaks could be used for this instance.

4. Yield (5 pts): Theoretical and percent yield should be provided with all step-by-step calculations.

5. Post-Lab Questions (30 pts): All the questions should be answered fully but succinctly. If drawings or mechanisms can help in the answer, they should be provided.

6. Lab Notebook (20 pts): Students should provide signed (by TA or instructor) carbon copies of their lab notebook where they should have a completed data table and any relevant observations.

7. Lab Technique/citizenship (10 pts): The lab should be returned to the condition in which you found it. Violations that are not attributable will be assessed to the whole class. Improper handling or use of equipment/chemicals will also cause deduction in points. 
TOTAL / 100 


\section{Stilbene Synthesis by Olefin Metathesis Reaction}

In a previous laboratory experiment, ${ }^{1}$ stilbene was synthesized via a Wittig reaction. The Wittig reaction is robust and widely-used in industrial and academic research labs. It is also a hallmark counterexample of a 'Green'2 process, and the reaction exhibits poor atom economy. ${ }^{3}$ That is, the mass of product divided by mass of 'wasted' Wittig reagent byproduct is low and can be less than unity, depending on the reaction. ${ }^{1}$ Catalytic methods offer an alternative. The primary advantage of a catalytic approach is the ability to generate many moles of product for each mole of catalyst (i.e. a good catalyst will have a high turnover number, TON $=$ mols substrate $/ \mathrm{mols}$ catalyst) and keep waste to a minimum. The multitude of synthetic possibilities and advantages rendered by tuning ligand structure - to change regiochemistry, stereochemistry, rate, and substrate scope - makes catalysis an attractive field of research. Stoichiometric (e.g. Wittig) and catalytic (e.g. metathesis) reactions have concomitant benefits and drawbacks. An overarching goal of the two stilbene synthesis experiments is to directly compare and contrast the two approaches.

Catalysts for olefin metathesis, particularly ruthenium $(R u)$-containing catalysts, have revolutionized synthetic chemistry. ${ }^{4}$ These catalysts have impacted pharmaceutical, ${ }^{5}$ natural products $^{6}$ and polymer chemistry. ${ }^{7}$ The development of olefin metathesis catalysts was awarded the 2005 Nobel Prize in Chemistry. ${ }^{8}$ In this experiment, you will be using a Ru olefin metathesis catalyst - commonly called Grubbs Catalyst, Second Generation or 'Grubbs 2' - to perform the homodimerization (or cross-metathesis) of styrene. The diastereoselectivity (cis/trans selectivity) of the metathesis transformation is different than the Wittig process. ${ }^{4}$

2<smiles>CCCCCCCCC=Cc1ccccc1</smiles>

\section{LIST OF REFERENCES}


1. Warner, J. C.; Anastas, P. T.; Anselme, J.-P. The Wittig Reaction in the Undergraduate Organic Laboratory. J. Chem. Ed. 1985, 62 (4), 346.

2. Noyori, R. Pursuing practical elegance in chemical synthesis. Chem. Commun. 2005, $14,1807-1811$

3. Trost, B. M. Atom Economy - A Challenge for Organic Synthesis: Homogeneous Catalysis Leads the Way. Angew. Chem. Int. Ed. Engl. 1995, 34 (3), 259-281.

4. Chatterjee, A.K.; Choi, T.-L.; Sanders, D.P.; Grubbs, R.H. A General Model for Selectivity in Olefin Cross Metathesis. J. Am Chem. Soc. 2003, 125 (37), 11360-11370.

5. Liu, M.; Mountford, S. J.; Richardson, R. R.; Groenen, M.; Holliday, N. D.; Thompson, P. E. Optically Pure, Structural, and Fluorescent Analogues of a Dimeric $\mathrm{Y}_{4}$ Receptor Agonist Derived by an Olefin Metathesis Approach. J. Med. Chem. 2016, 59 (13), 60596069 .

6. Lad, N. P.; Kulkarni, S.; Sharma, R.; Mascarenhas, M.; Kulkarni, M. R.; Pandit, S. S. Piperlongumine derived cyclic sulfonamides (sultams): Synthesis and in vitro exploration for therapeutic potential against HeLa cancer cell lines. Eur. J. Med. Chem. 2017, 126, 870-878.

7. Sinclair, F.; Alkattan, M.; Prunet, J.; Shaver, M. P. Olefin Cross Metathesis and RingClosing Metathesis in Polymer Chemistry. Polym. Chem. 2017, 8 (22), 3385-3398.

8. Grubbs, R. H. Olefin-Metathesis Catalysts for the Preparation of Molecules and Materials (Nobel Lecture). Angew. Chem. Int. Ed. 2006, 45 (23), 3760-3765. 


\section{Experimental - Day 1}

Charge a $20 \mathrm{~mL}$ scintillation vial with a magnetic stir bar, Grubbs $2(14.80 \mathrm{mg}, 0.017 \mathrm{mmol})$ and dichloromethane $\left(\mathrm{CH}_{2} \mathrm{Cl}_{2}\right)(10 \mathrm{~mL}, 0.157 \mathrm{mmol})$. Next, add the styrene* $(0.2 \mathrm{~mL}, 1.74 \mathrm{mmol})$, fit the scintillation vial with a polymer cone or foil backed cap and place on stir plate. Let the reaction stir for 1 hour, and then remove the solvent in vacuo.

Prepare a mini silica column, Figure 1. First, loosely plug one end of a pipet $(8 \times 142 \mathrm{~mm})$ with a piece of cotton or glass wool. Next, fill pipet with silica to a height of $\sim 5 \mathrm{~cm}$. Add the hexanes mobile phase to the top of the mini column and use a volumetric pipette bulb to push the mobile phase onto the column. Use gradual pressure changes to move the solvent without cracking the silica gel stationary phase; this can take practice and patience.

Re-dissolve the vial contents in minimal $\mathrm{CH}_{2} \mathrm{Cl}_{2}(0.5-1.0 \mathrm{~mL})$. Pipet this solution onto the silica plug, trying not to disturb the wet silica. After loading the reaction solution onto the column, flush the plug with excess hexanes $(\sim 25 \mathrm{~mL})$ to remove the stilbene, collecting in a $100 \mathrm{~mL}$ round bottom flask. A shorter column $(\sim 2 \mathrm{~cm})$ can be eluted with a smaller amount $(\sim 10 \mathrm{~mL})$ of hexanes, but loading in minimal $\mathrm{CH}_{2} \mathrm{Cl}_{2}$ is critical. Remove the solvent in vacuo and collect ${ }^{1} \mathrm{H}$ NMR, IR spectra and melting point. Determine the cis-/trans- ratio of the product, turnover number (TON) and maximum turnover frequency (TOF) of the reaction.

*Styrene from a chemical supplier contains an inhibitor. The inhibitor may not be disruptive to the metathesis reaction, but it was removed prior to the lab period. The inhibitor was removed by stirring for $5 \mathrm{~min}$ a mixture of $3 \mathrm{~g}$ of alumina for every $20 \mathrm{~mL}$ of styrene and gravimetrically filtering the slurry through qualitative filter paper. 


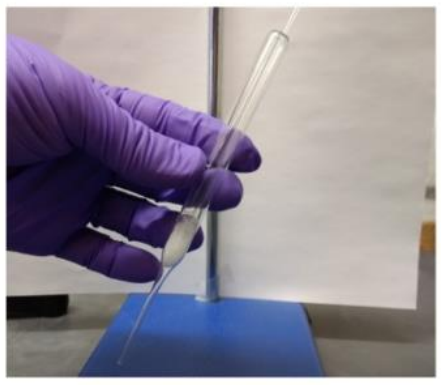

A

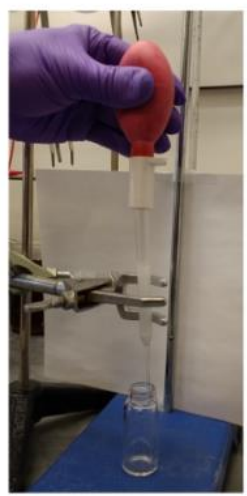

D

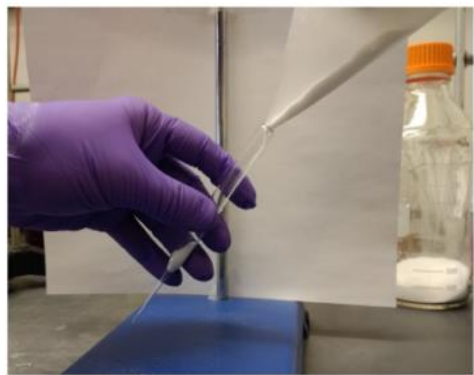

B

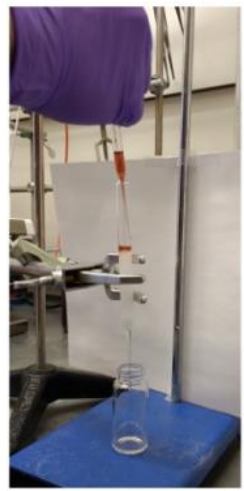

$\mathrm{E}$

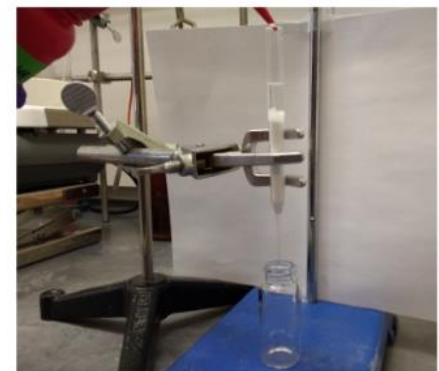

C

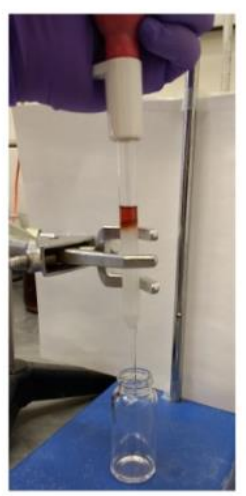

$\mathrm{F}$

Figure 1: (a) Insertion of a glass wool into a glass pipette $(8 \times 142 \mathrm{~mm})$ with a 9 inch Pasteur pipette; (b) Loading of dry silica into the glass pipette with weigh paper; (c) Wetting of the silica with hexanes; (d) Application of pressure with a volumetric pipette bulb to elute the solvent; (e) Loading the product mixture on to the wet silica column; (f) Elution of product with firm, constant pressure from pipette bulb.

\section{Day 2}

Form a hypothesis, design a modification of the experiment and reach a conclusion supported by your data. Possible modifications may be to test the turnover limits of the reaction by reducing the catalyst loading, or by changing the reaction time, temperature and/or concentration of reagents. A more complex study of reaction conditions may be screened if you pair with your presentation partner(s) to design your experiments. 


\section{Questions}

1. What are the advantages of synthesizing stilbene with metathesis vs Wittig? Disadvantages?

2. What is the cis/trans ratio produced by metathesis and how does it compare to the Wittig reaction?

3. Draw the catalytic cycle that produces stilbene. Where is the stereochemistry set (i.e. at what point does the product become cis- or trans-?

4. A properly-designed catalyst can produce non-thermodynamic distributions of products (i.e. a kinetic or Curtin-Hammett distribution of products). Is this metathesis reaction thermodynamically or kinetically controlled? Can you design an experiment to test your answer?

5. Is this catalyst a good choice for olefin metathesis? (hint: take a look at your TON and TOF). Hit the literature, what other catalyst might you suggest for the metathesis of styrene? 